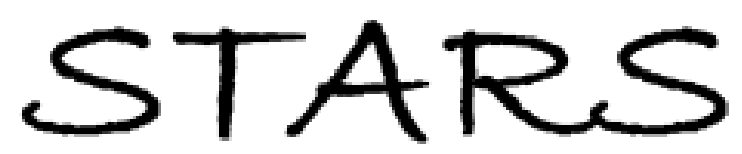

University of Central Florida

STARS

2018

\title{
Analysis of Heat Transfer on Turbulence Generating Ribs using Dynamic Mode Decomposition
}

\author{
Michael Elmore \\ University of Central Florida
}

Part of the Aerodynamics and Fluid Mechanics Commons

Find similar works at: https://stars.library.ucf.edu/etd

University of Central Florida Libraries http://library.ucf.edu

This Masters Thesis (Open Access) is brought to you for free and open access by STARS. It has been accepted for inclusion in Electronic Theses and Dissertations by an authorized administrator of STARS. For more information, please contact STARS@ucf.edu.

\section{STARS Citation}

Elmore, Michael, "Analysis of Heat Transfer on Turbulence Generating Ribs using Dynamic Mode Decomposition" (2018). Electronic Theses and Dissertations. 6204.

https://stars.library.ucf.edu/etd/6204

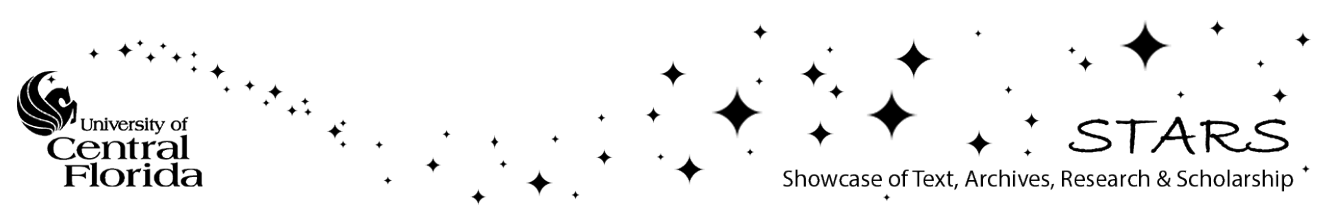




\title{
ANALYSIS OF HEAT TRANSFER ON TURBULENCE GENERATING RIBS USING DYNAMIC MODE DECOMPOSITION
}

by

\section{MICHAEL ELMORE}

B.S. Aerospace Engineering, University of Michigan, 2012

\begin{abstract}
A thesis submitted in partial fulfilment of the requirements for the degree of Master of Science in Aerospace Engineering in the Department of Mechanical and Aerospace Engineering in the College of Engineering and Computer Science at the University of Central Florida Orlando, Florida
\end{abstract}

\section{Fall Term 2018}

Major Professor: Jayanta Kapat 
(c) 2018 Michael Elmore 


\begin{abstract}
Ducts with turbulence-promoting ribs are common in heat transfer applications. This study uses a recent modal extraction technique called Dynamic Mode Decomposition (DMD) to determine mode shapes of the spatially and temporally complex flowfield inside a ribbed duct. One subject missing from current literature is a method of directly linking a mode to a certain engineering quantity of interest. Presented is a generalized methodology for producing such a link utilizing the data from the DMD analysis. Theory suggests exciting the modes which are identified may cause the flow to change in such a way to promote the quantity of interest, in this case, heat transfer. This theory is tested by contouring the walls of the duct by the extracted mode shapes.

The test procedure is taken from an industrial perspective. An initial, unmodified geometry provides a baseline for comparison to later contoured models. The initial case is run as a steady-state Reynolds-Averaged Navier-Stokes model. Large-Eddy Simulation generates the necessary data for the DMD analysis. Several mode shapes extracted from the flow are applied to the duct walls and run again in the RANS model, then compared to the baseline, and their relative performance examined.
\end{abstract}


Dedicated to my Mom and Dad. Their immeasurable kindness and relentless passion to build better things for those around them have given me continual opportunity. "You better get an education!" Dad always said. My most valuable education was acquired not at some far institution, but in their own home. 


\section{ACKNOWLEDGMENTS}

A special thanks to:

Dr. Kapat for giving me the opportunity to earn the graduate degree I wanted for so many years, and his guidance in this great challenge through courses, labwork, and beyond.

The fine folks at the CATER Lab for putting up with me. 


\section{TABLE OF CONTENTS}

LIST OF FIGURES . . . . . . . . . . . . . . . . . . . . . . xi

LIST OF TABLES . . . . . . . . . . . . . . . . . . . . . . . . . . . . . . . .

LIST OF SYMBOLS . . . . . . . . . . . . . . . . . . . Xx

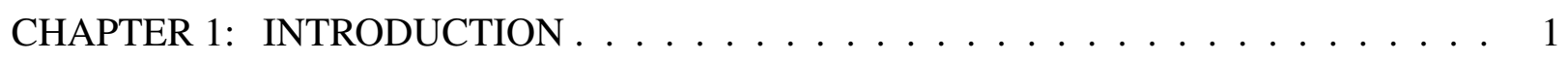

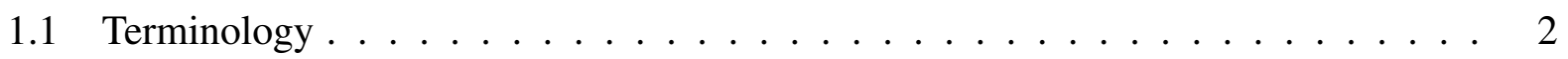

1.2 Goal of This Research . . . . . . . . . . . . . . . . 3

CHAPTER 2: LITERATURE REVIEW . . . . . . . . . . . . . . . . . . . 5

2.1 Review of IDC Literature . . . . . . . . . . . . . . . . . . 5

2.2 Review of DMD Literature . . . . . . . . . . . . . . . . . . 9

CHAPTER 3: METHODOLOGY . . . . . . . . . . . . . . . . . 12

3.1 Physics Introduction $\ldots \ldots \ldots \ldots \ldots$

3.1 .1 Pressure Loss/Friction Factor . . . . . . . . . . . . . . . 12

3.1.2 Heat Transfer Coefficient . . . . . . . . . . . . . . 13

3.2 CFD Model Introduction . . . . . . . . . . . . . . . . . . 14 


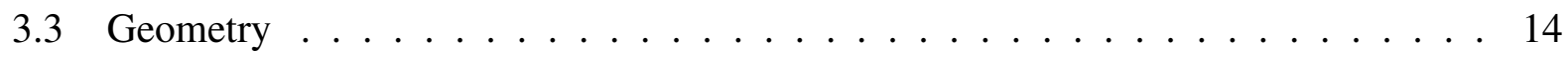

3.4 Mesh Generation . . . . . . . . . . . . . . . . . . . . 15

3.5 RANS Numerical Setup . . . . . . . . . . . . . . . . . 16

3.5.1 Discretization . . . . . . . . . . . . . . . . . . . . . 17

3.5.2 Convergence Criterion $\ldots \ldots \ldots \ldots \ldots$

3.5.3 Near-Wall Resolution . . . . . . . . . . . . . . . . . . . . . . . . 17

3.6 LES Numerical Setup . . . . . . . . . . . . . . . . . . . . . . . . . . . 17

3.6.1 Discretization . . . . . . . . . . . . . . . . . . . . . . 18

$3.6 .2 \quad$ Subgrid Scale Model . . . . . . . . . . . . . . . . . . . . . . . . 18

3.6.3 Evaluating Statistical Stationarity $\ldots \ldots \ldots . \ldots 20$

3.6 .4 Grid Resolution . . . . . . . . . . . . . . . . . . . . . . . . . . . . . . 22

3.6 .5 Near-Wall Resolution . . . . . . . . . . . . . . . . . . . . . . . . . . . . 23

3.6 .6 Turbulent Scales . . . . . . . . . . . . . . . . . . . . . . . . . 24

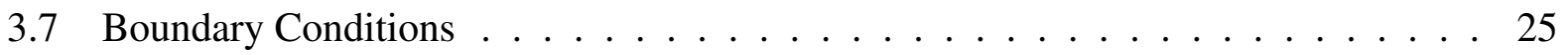

3.8 DMD Introduction $\ldots \ldots \ldots \ldots \ldots \ldots \ldots$

$3.8 .1 \quad$ DMD Algorithm Process . . . . . . . . . . . . . . . . . 31

3.8.2 Rank Truncation Methodology . . . . . . . . . . . . . . . . . . . . 34 
3.8.3 DMD Mode Amplitude and Frequency . . . . . . . . . . . . . . . . . 34

3.8.4 Modal Contribution to Engineering Quantities . . . . . . . . . . . . 36

3.9 DMD Convergence Study . . . . . . . . . . . . . . . . . . . 37

3.9.1 Parameter Limits . . . . . . . . . . . . . . . . . . . . . . . 37

3.9.2 Convergence Assessment Introduction . . . . . . . . . . . . . . 38

3.9.3 Dependence on Number of Retained Ranks . . . . . . . . . . . . . . . 39

3.9 .4 Ritz Value Convergence $\ldots \ldots \ldots$. . . . . . . . . . . . . 43

3.9.5 Modal Amplitude Convergence . . . . . . . . . . . . . . . . . 47

3.9.6 Reconstruction Residual Convergence . . . . . . . . . . . . . . . . 51

3.9 .7 Reconstruction Visualization . . . . . . . . . . . . . . . . 52

CHAPTER 4: RESULTS . . . . . . . . . . . . . . . . . . . . 54

$4.1 \quad$ RANS Baseline Case Results . . . . . . . . . . . . . . . . . . . . 55

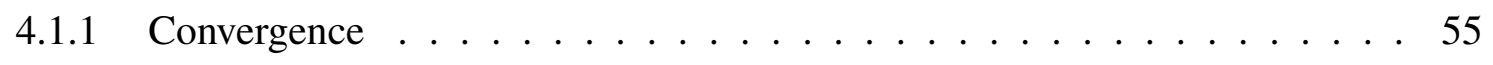

4.1 .2 Pressure Drop . . . . . . . . . . . . . . . 56

4.1.3 Flowfield Features and Description $\ldots \ldots \ldots$

$4.1 .4 \quad$ Heat Transfer . . . . . . . . . . . . . . . . . . . . 58

$4.2 \quad$ LES Case Results . . . . . . . . . . . . . . . . . . . . . . . . . . 59 
$4.2 .1 \quad$ Statistical Stationarity $\ldots \ldots \ldots \ldots$

$4.2 .2 \quad$ Pressure Drop . . . . . . . . . . . . . . . . . . . 60

4.2.3 Flowfield Features and Description $\ldots \ldots \ldots$. . . . . . . 61

4.2 .4 Heat Transfer . . . . . . . . . . . . . . . . . . . 63

4.3 DMD Results . . . . . . . . . . . . . . . . . . . . . . . 64

4.3.1 Modes Selected for Morphing _ . . . . . . . . . . . . . 70

4.3.2 Spectral Comparison . . . . . . . . . . . . . . 75

4.4 Modified RANS Case Geometry . . . . . . . . . . . . . . . . . . . 77

4.5 Modified RANS Case Results _ . . . . . . . . . . . . . . . . . 81

$4.5 .1 \quad$ Pressure Drop . . . . . . . . . . . . . . . . . 81

4.5 .2 Heat Transfer . . . . . . . . . . . . . . . . . . . . . . 81

4.5.3 Flowfield Features and Description $\ldots \ldots \ldots \ldots$

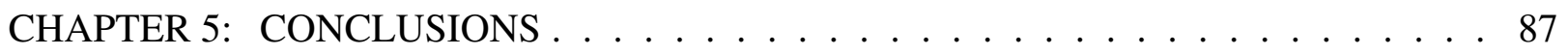

$5.1 \quad$ Future Work . . . . . . . . . . . . . . . . . . . . . . . . . . 89

5.1.1 Sensitivity to Morphed Displacement Magnitude . . . . . . . . . . . . 89

5.1 .2 Verification of Results using LES . . . . . . . . . . . . . . 90

5.1.3 Less Expensive DMD Data Simulations . . . . . . . . . . . . . . 91 
APPENDIX A: ADDITIONAL FIGURES . . . . . . . . . . . . . . . . 93

APPENDIX B: MORPHED CASE POST-PROCESSING . . . . . . . . . . . . . . 105

APPENDIX C: NOTES ON THE TKE RESOLUTION RATIO . . . . . . . . . . . . . . 117

APPENDIX D: DETAILED COMPUTATIONAL TIME USAGE . . . . . . . . . . . . . 120

APPENDIX E: DMD CODE NOTES . . . . . . . . . . . . . . . . . . . . . . . 124

APPENDIX F: DMD CODE . . . . . . . . . . . . . . . . . . . . . . . . 127

LIST OF REFERENCES . . . . . . . . . . . . . . . . . . . . . . . . . . . . . . . . . . 129 


\section{LIST OF FIGURES}

Figure 1.1: IDC Terminology . . . . . . . . . . . . . . . . 2

Figure 3.1: RANS baseline and LES case geometry . . . . . . . . . . . . . . 14

Figure 3.2: Computational mesh, center cut . . . . . . . . . . . . . . 15

Figure 3.3: Computational mesh, near rib . . . . . . . . . . . . . . 16

Figure 3.4: Sampling probe locations for statistics . . . . . . . . . . . . . 22

Figure 3.5 : Boundary condition diagram $\ldots \ldots \ldots \ldots$

Figure 3.6: Example point probe grid . . . . . . . . . . . . . . . . 30

Figure 3.7: Outline of DMD Domain Volume . . . . . . . . . . . . . . . 30

Figure 3.8: Normalized Singular Values _. . . . . . . . . . . . . . . . . 34

Figure 3.9: Ritz value convergence for various retained ranks . . . . . . . . . . . 41

Figure 3.10 Spectral convergence for various retained ranks . . . . . . . . . . . 42

Figure 3.11 Residual convergence for various retained ranks . . . . . . . . . . . . . 42

Figure 3.12Ritz value convergence, $N=1 \ldots \ldots \ldots$. . . . . . . . . . 45

Figure 3.13Ritz value convergence, $N=10 \ldots \ldots \ldots$

Figure 3.14 Ritz value convergence, $\Delta t=80 \mu \mathrm{s} \ldots \ldots \ldots$. . . . . . . 46 
Figure 3.15Ritz value convergence, $\Delta t=20 \mu \mathrm{s} \ldots \ldots \ldots \ldots$

Figure 3.16 Ritz value convergence, all cases $\ldots \ldots \ldots$. . . . . . . . . . . 47

Figure 3.17DMD amplitude convergence, $N=5 \ldots \ldots \ldots$

Figure 3.18DMD amplitude convergence, $N=10 \ldots \ldots \ldots$. . . . . . 49

Figure 3.19DMD amplitude convergence, $\Delta t=40 \mu \mathrm{s} \ldots \ldots \ldots \ldots$

Figure 3.20DMD amplitude convergence, $\Delta t=20 \mu \mathrm{s} \ldots \ldots \ldots \ldots$

Figure 3.21DMD reconstruction residuals $\ldots \ldots \ldots \ldots \ldots$

Figure 3.22Temperature Isosurface Comparison $\ldots \ldots \ldots$. . . . . . . . . 53

Figure 3.23Rank-Temperature Isosurface Comparison . . . . . . . . . . . . . . 53

Figure 4.1: Heat transfer convergence, RANS baseline case . . . . . . . . . . 55

Figure 4.2: Residual convergence, RANS baseline case . . . . . . . . . . . . 56

Figure 4.3: Flow structure, RANS baseline case $\ldots \ldots \ldots$. . . . . . . . 57

Figure 4.4: Mid-rib Vorticity, RANS baseline case . . . . . . . . . . . . . 58

Figure 4.5: Nusselt ratio, RANS baseline case, top view. . . . . . . . . . . . . . . . . 59

Figure 4.6: Nusselt ratio, RANS baseline case, front and rear view $\ldots \ldots$. . . . . . 59

Figure 4.7: Velocity Magnitude Time History . . . . . . . . . . . . . . . . . 60

Figure 4.8: Pressure drop, RANS baseline and LES cases $\ldots \ldots$. . . . . . . . . . 61 
Figure 4.9: Flow structure, LES case . . . . . . . . . . . . . . . . . . 62

Figure 4.10Streamline Impingement Comparison, LES vs RANS ． . . . . . . . . . . 63

Figure 4.11 Nusselt ratio, LES case, top view . . . . . . . . . . . . . . . . . . 64

Figure 4.12Nusselt ratio, LES case, front and rear view . . . . . . . . . . . . . . . 64

Figure 4.13Normalized heat transfer for each DMD mode . . . . . . . . . . . . . . 67

Figure 4.14Normalized heat transfer for each DMD mode . . . . . . . . . . . . . . 67

Figure 4.15Difference in normalized heat transfer for each DMD mode . . . . . . . . . 68

Figure 4.16Diagram of DMD surface values . . . . . . . . . . . . . . . 68

Figure 4.17Reconstructed Nusselt Ratio Difference, Mode 7-5 . . . . . . . . . . . . 69

Figure 4.18Reconstructed Nusselt Ratio Difference, Mode 17-15 . . . . . . . . . . . . . 69

Figure 4.19Reconstructed Nusselt Ratio Difference, Mode 25-23 . . . . . . . . . . . . . 70

Figure 4.20Near wall DMD mode shape, mode 13, case D1 . . . . . . . . . . . . . 72

Figure 4.21Near wall DMD mode shape, mode 33, case D2 . . . . . . . . . . . . 72

Figure 4.22Near wall DMD mode shape, mode 17 , case D3 . . . . . . . . . . . 73

Figure 4.23Near wall DMD mode shape, mode 99, case D4 . . . . . . . . . . . 73

Figure 4.24Near wall DMD mode shape, mode 7, case D5 . . . . . . . . . . . . . . 74

Figure 4.25Near wall DMD mode shape, mode 25, case D6 . . . . . . . . . . . . 74 
Figure $4.26 \mathrm{DMD}$ Spectrum $\ldots \ldots \ldots \ldots \ldots \ldots \ldots$

Figure 4.27 Structures from mode $7 \ldots \ldots$. . . . . . . . . . . . 77

Figure 4.28Surface Deformation Along Span . . . . . . . . . . . . . . . . 80

Figure 4.29Heat Transfer Changes Relative to Baseline RANS . . . . . . . . . . . . 84

Figure A.1:Viscous Wall Distance Values . . . . . . . . . . . . . . . . . . . . . 94

Figure A.2:Ritz value convergence, $N=3 \ldots \ldots \ldots \ldots$

Figure A.3:Ritz value convergence, $N=5 \ldots \ldots \ldots \ldots \ldots$

Figure A.4:Ritz value convergence, $\Delta t=40 \mu s \quad \ldots \ldots \ldots 6$

Figure A.5:Ritz value convergence, $\Delta t=160 \mu s \ldots \ldots \ldots 6$

Figure A.6:DMD amplitude convergence, $N=1 \ldots \ldots \ldots$. . . . . . 97

Figure A.7:DMD amplitude convergence, $N=3 \ldots \ldots \ldots$

Figure A.8:DMD amplitude convergence, $\Delta t=80 \mu s \ldots \ldots \ldots$

Figure A.9:DMD amplitude convergence, $\Delta t=160 \mu \mathrm{s} \ldots \ldots \ldots 98$

Figure A.10Heat Transfer for Individual DMD modes, per wall $\ldots \ldots \ldots 99$

Figure A.1 Heat Transfer for Individual DMD modes, per wall $\ldots \ldots \ldots$

Figure A.122D mode shape, Mode 13, front view. . . . . . . . . . . . . . . . . 100

Figure A.133D mode shape, Mode 13, rear view. . . . . . . . . . . . . . . . . . 101 
Figure A.14BD mode shape, Mode 33, front view. . . . . . . . . . . . . . . . . 101

Figure A.153D mode shape, Mode 33, rear view. . . . . . . . . . . . . . . . . . 101

Figure A.16D mode shape, Mode 17, front view. . . . . . . . . . . . . . . . . . . 102

Figure A.17D mode shape, Mode 17, rear view. . . . . . . . . . . . . . . . . . 102

Figure A.183D mode shape, Mode 99, front view. . . . . . . . . . . . . . . . . . 102

Figure A.198D mode shape, Mode 99, rear view. . . . . . . . . . . . . . . . . . . 103

Figure A.202D mode shape, Mode 7, front view. . . . . . . . . . . . . . . . 103

Figure A.2 BD mode shape, Mode 7, rear view. . . . . . . . . . . . . . . . . 103

Figure A.222D mode shape, Mode 25, front view. . . . . . . . . . . . . . . . . . . 104

Figure A.233D mode shape, Mode 25, rear view. . . . . . . . . . . . . . . . . . . 104

Figure B.1:Nusselt ratio, case D1, front and rear view . . . . . . . . . . . 106

Figure B.2:Nusselt ratio, case D2, front and rear view . . . . . . . . . . 106

Figure B.3:Nusselt ratio, case D3, front and rear view . . . . . . . . . . . . 107

Figure B.4:Nusselt ratio, case D4, front and rear view . . . . . . . . . . . . 107

Figure B.5:Nusselt ratio, case D5, front and rear view . . . . . . . . . . . . . 107

Figure B.6:Nusselt ratio, case D6, front and rear view . . . . . . . . . . . . 108

Figure B.7:Nusselt ratio, case D1, front and rear view . . . . . . . . . . . . 108 
Figure B.8:Nusselt ratio, case D2, front and rear view . . . . . . . . . . . . 108

Figure B.9:Nusselt ratio, case D3, front and rear view . . . . . . . . . . . . . . 109

Figure B.10Nusselt ratio, case D4, front and rear view . . . . . . . . . . . . . 109

Figure B.1 1Nusselt ratio, case D5, front and rear view . . . . . . . . . . . . . 109

Figure B.12Nusselt ratio, case D6, front and rear view . . . . . . . . . . . . . 110

Figure B.13streamlines/Q criterion, case D1 . . . . . . . . . . . . . . . 110

Figure B.14Streamlines/Q criterion, case D2 . . . . . . . . . . . . . 110

Figure B.15Streamlines/Q criterion, case D3 . . . . . . . . . . . . 111

Figure B.16Streamlines/Q criterion, case D4 . . . . . . . . . . . 111

Figure B.17Streamlines/Q criterion, case D5 . . . . . . . . . . . . . 111

Figure B.18Streamlines/Q criterion, case D6 . . . . . . . . . . . . . . 112

Figure B.19Vorticity, case D1, midrib section . . . . . . . . . . . . . . 112

Figure B.20Vorticity, case D2, midrib section . . . . . . . . . . . . . 112

Figure B.21Vorticity, case D3, midrib section . . . . . . . . . . . . 113

Figure B.22Vorticity, case D4, midrib section . . . . . . . . . . . . 113

Figure B.23Vorticity, case D5, midrib section . . . . . . . . . . . . 113

Figure B.24Vorticity, case D6, midrib section . . . . . . . . . . . . . . 114 
Figure B.25surface streamlines, case D1, front and rear view . . . . . . . . . . . 114

Figure B.26Surface streamlines, case D2, front and rear view . . . . . . . . . . . 114

Figure B.27Surface streamlines, case D3, front and rear view . . . . . . . . . . 115

Figure B.28Surface streamlines, case D4, front and rear view . . . . . . . . . . 115

Figure B.29surface streamlines, case D5, front and rear view . . . . . . . . . 115

Figure B.30Surface streamlines, case D6, front and rear view . . . . . . . . . 116

Figure C.1:TKE Resolution Ratio Violation . . . . . . . . . . . . . . . . . . . 119 


\section{LIST OF TABLES}

Table 2.1: Parameters studied in literature . . . . . . . . . . . . . 8

Table 3.1: Geometric parameters for this study . . . . . . . . . . . . 15

Table 3.2: Estimated DNS run metrics . . . . . . . . . . . . . . 25

Table 3.3: RANS cases boundary conditions . . . . . . . . . . . . . 25

Table 3.4: LES case boundary conditions . . . . . . . . . . . . . 26

Table 3.5: Number of timesteps in DMD convergence tests . . . . . . . . . . . 39

Table 3.6: Number of ranks in DMD convergence tests . . . . . . . . . . . . . 40

Table 4.1: Selected DMD Modes for Morphing . . . . . . . . . . . . . . 71

Table 4.2: Frequencies of Modes used in Morphing . . . . . . . . . . . . . 76

Table 4.3: DMD Field Morphing Scale Factors and Displacement Metrics . . . . . . . 79

Table 4.4: Morphed Cases Friction Factor Comparison . . . . . . . . . . . . . 81

Table 4.5: Heat Transfer Changes Relative to Baseline RANS . . . . . . . . . . . 85

Table D.1: RANS Cases Computational Cost . . . . . . . . . . . . . . . 121

Table D.2: LES Case Computational Cost . . . . . . . . . . . . . . . . . 121

Table D.3: DMD Convergence Run Computational Cost . . . . . . . . . . . . . . . . 122 
Table D.4: DMD Maximum Rank Run Computational Cost . . . . . . . . . . . . . . . . 122

Table D.5: DMD Convergence Run Memory Requirements . . . . . . . . . . . . . . 123 


\section{LIST OF SYMBOLS}

A Best-fit linear time transformation operator matrix

$A^{\prime} \quad$ Low-dimension best-fit linear transformation operator ma-

trix

$A_{h} \quad$ Area transferring heat

$A_{r} \quad$ Total number of reversals

$\alpha \quad$ Rib angle

AR Channel aspect ratio

$b_{k} \quad k$ th initial condition vector

$C_{w} \quad$ WALE model constant

CFL Courant number

$D_{h} \quad$ Hydraulic diameter

$\Delta \quad$ Filter scale

$\Delta s \quad$ Wall distance/grid spacing

$\Delta t \quad$ Time step

$\Delta x \quad$ Grid spacing in $\mathrm{x}$ dimension

$e \quad$ Rib height

$f \quad$ Darcy friction factor

G Filter kernel

H Channel height

$h \quad$ Heat transfer coefficient

$h_{i j} \quad$ Reverse arrangement counter

$k_{f} \quad$ Fluid thermal conductivity

$k_{\text {res }} \quad$ Resolved turbulent kinetic energy

$k_{\text {sgs }} \quad$ Sub-grid scale turbulent kinetic energy 


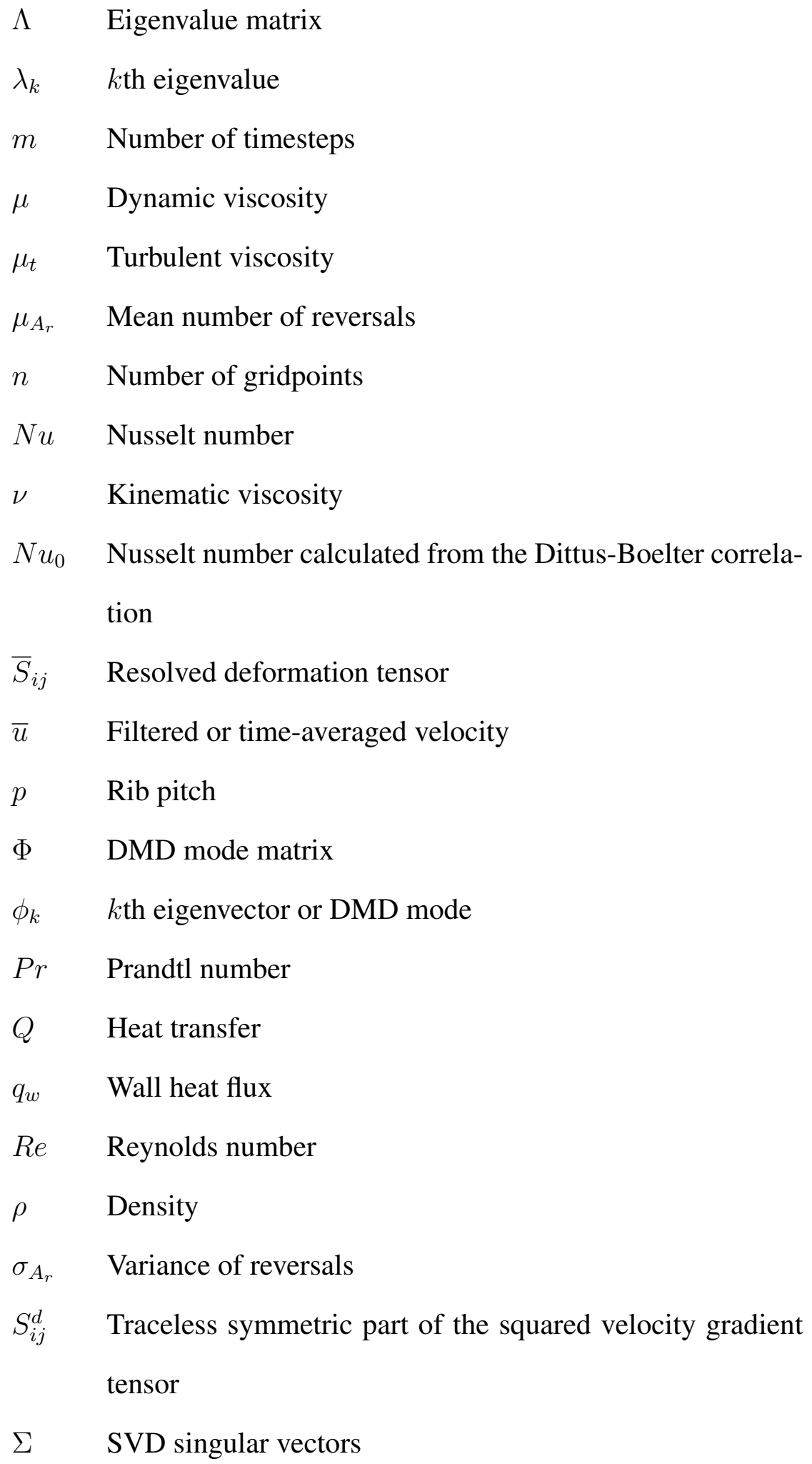




$\begin{array}{ll}T_{b} & \text { Bulk temperature } \\ T_{w} & \text { Wall temperature } \\ \tau_{i j} & \text { Residual stress tensor } \\ \tau_{w} & \text { Wall shear stress } \\ T_{p} & \text { Partially reconstructed temperature field } \\ T_{r} & \text { Reconstructed temperature field } \\ U & \text { SVD left-singular vectors } \\ u^{\prime} & \text { Velocity fluctuation } \\ V & \text { SVD right-singular vectors } \\ V_{i} & \text { Volume surrounding gridpoint } i \\ V^{*} & \text { Conjugate transpose of V } \\ W^{\prime} & \text { Channel width } \\ W_{v} & \text { Eigenvector matrix } \\ X & \text { Matrix of snapshots } \\ X^{+} & \text {Moore-Penrose pseudoinverse of } X \\ x^{+} & \text {Streamwise distance normalized by viscous length } \\ y^{+} & \text {Wall distance normalized by viscous length } \\ z^{+} & \text {Spanwise distance normalized by viscous length } \\ \end{array}$




\section{CHAPTER 1: INTRODUCTION}

In an ever-present quest for higher efficiency, modern gas turbines face increasing firing temperatures, which can exceed $1700 \mathrm{~K}$. These high temperatures from combustion are imposed on the machine's turbine. Turbines are often built from metals which melt below the temperature of the gas in which they operate, making active cooling necessary to attain better performance. Numerous cooling methods are employed simultaneously on modern blades, but one of the most common is an internal cooling passage. This internal duct cooling (IDC) is vital to the removal of heat from the blade, allowing the metal to survive the hot firing gas. The internal duct can come in many shapes and sizes, and often includes turbulence-generating ribs (turbulators) to enhance gas mixing near the boundary layer, increasing heat transfer performance. The gas moving through the duct comes from a bleed on the compressor output, which means the duct's presence reduces the maximum pressure of the compressor. This loss is undesirable, which places a constraint on the duct geometry. The duct must simultaneously perform enough cooling to ensure the blade survives thousands of hours of usage in hot gasses while not causing too great of a pressure loss from the compressor. The basic features of IDC are vital to engine performance and efficiency, and are under constant improvement and enhancement. Through most of current gas turbine research, this improvement is found via parametric studies, i.e. running a sweep of a few independent dimensions on a geometry and determining what works well. With the steady rise of nearly unlimited flexibility in additive manufacturing methods . One such method is Dynamic Mode Decomposition (DMD). This study hopes to use the data-driven decomposition method to modify a surface in an otherwise unintuitive way to increase heat transfer. This methodology, though proposed for use on gas turbines, can be applied to nearly any engineering scenario involving fluid flow. 


\subsection{Terminology}

The geometry and associated terminology for a general IDC geometry are shown figure 1.1.

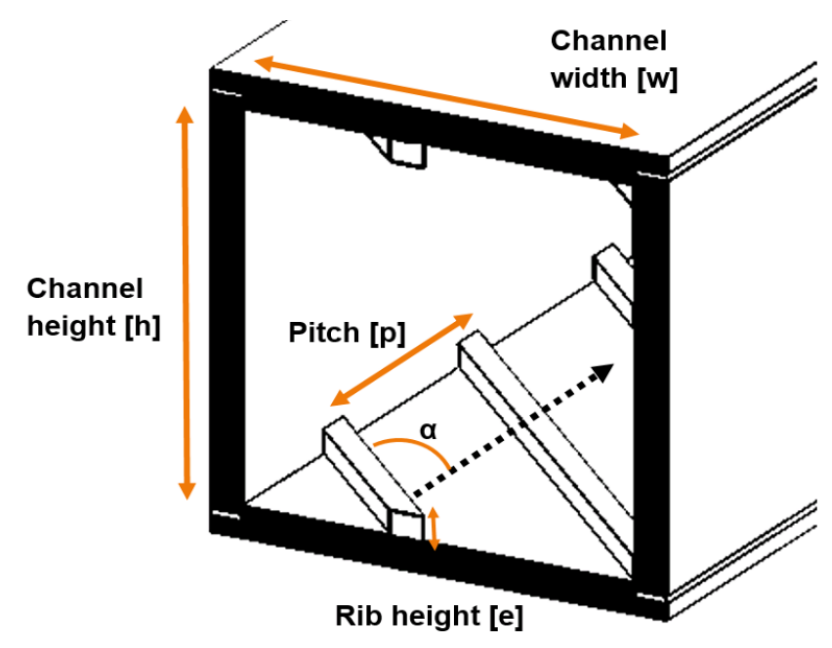

Figure 1.1: IDC Terminology

Visible are:

- Channel height $(H)$

- Channel width $(W)$

- Rib height (e)

- Rib angle $(\alpha)$

- Rib pitch $(p)$

These values are often formed to make various non-dimensional parameters such as: 
- $e / D_{h}$ - rib height to hydraulic diameter ratio

- $p / e$ - pitch to rib height ratio

- $W / H$ or $A R$ - channel aspect ratio

\subsection{Goal of This Research}

This article investigates the heat transfer from a rib turbulator by exploring the turbulent wake using DMD. A square channel with 45 degree ribs is used as the starting geometry at Reynolds number 32,400 . By analyzing the modal turbulent structures in the wake via DMD, it is possible to determine which modes are important to heat transfer. The shape of these modes are used to deform the rib surface in an effort to excite the modes and increase heat transfer. This will be accomplished using several steps:

- Develop a baseline IDC industrial heat transfer model with RANS

- Evaluation of the model above with Large Eddy Simulation

- Perform DMD on sampled LES data

- Analyze the DMD data to identify modes which enhance heat transfer

- Modify the topology of the geometry to enhance the heat transfer from the mode shape information

- Perform an additional RANS simulation to determine if an increase in heat transfer is realized 
These steps are intended to mirror what an industrial engineer attempting to improve a product might do. Combining this with the theoretical background of DMD, along with an algorithm to link mode shapes to heat transfer changes, aims to provide good academic and industrial value. 


\section{CHAPTER 2: LITERATURE REVIEW}

The research presented in this thesis attempts to bridge two pools of knowledge within the community; that of IDC physics and usage of the DMD algorithm. Considering this, the relevant research performed by other authors is presented in two separate sections.

\subsection{Review of IDC Literature}

Research on improving the performance of IDC spans across the globe and decades. Numerous investigations have been performed by modifying different aspects of the IDC channel, whose parameters are generally taken to be along those introduced in figure 1.1. Much foundational work has been performed by Han et al. [18] whom investigated basic aspects of rib parameters such as rib height (e), pitch (p), angle $(\alpha)$, as well as parameters of the duct itself such as aspect ratio (AR). Their work shows even basic ribs can increase heat transfer by 2-5 times over smooth channels. They also showed that ribs between $45^{\circ}$ and $60^{\circ}$ show superior heat transfer than $90^{\circ}$ for similar pressure losses. The geometry used in this analysis closely mirrors one of theirs. Park et al. [34] studied modification of the channel aspect ratio equipped with different rib heights and geometric patterns, and exemplifies the parametric nature of IDC research. They found for wide aspect ratios, ribs at $30-45^{\circ}$ showed better heat transfer performance. Differing aspect ratios with rotation was studied by Han et al. [17]. Simpler transverse ribs make up a large number of studies, however more complex patterns in V, W, and M arrangements appear as well in Singh et al. [52], Kunstmann et al. [24], Write et al. [60], and Dritselis [12]. Singh et al. [52] showed that V shaped ribs can perform well across many Re, providing average Nusselt number ratios $\frac{N u}{N u_{0}}$ between 2.5-3.0. Ribs with W shapes and standard transverse ribs seemed to do slightly worse

for various Re, with $\frac{N u}{N u_{0}}=2-2.5$. However it was shown by Wright et al. [60] that by breaking up 
rib patterns, heat transfer can further improve. In addition to modifying the pattern of ribs, some researchers choose to modify ribs in a local (but still parametric) manner; ribs can appear with truncations as in Xie et al. [61] and Forsyth et al. [14], and with other geometric features like dimples as in Mhetras et al. [30]. The rib geometry is also modified from a square cross-section to trapezoids in Ali et al. [3], circles in Dritselis [12], and wedge features in Valentino et al. [57]. Table 2.1 displays a breakdown of a selection of researched geometries. This is just a small glimpse of the great expanse of studies done over the past many years. Upon review of this table, it should be evident that the geometric possibilities of rib and duct configurations extend wide and far. Advances in additive manufacturing make the complexity of possible designs almost limitless. The parametric nature of the above studies does not fully utilize this power, as the features used are defined by small numbers of geometric parameters. Increasing the number of parameters by surface contouring may bring about further enhancements on top of the few geometric parameters utilized, which is the subject of this research.

The tools used in IDC research often boil down to one or many of several choices. The list below provides keys for the acronyms in the Methods column of table 2.1. Experimental investigations can include:

- Pressure drop under cold/hot flow (PD)

- Heat transfer determination using electrically heated metal walls (HT)

- Heat transfer distribution using Thermochromic Liquid Crystals (TLC)

- Flow quantification using hotwire anemometers (HW)

- Flow quantification using Laser Doppler Velocimetry (LDV) and/or Particle Image Velocimetry (PIV)

Numerical investigations are often performed using RANS or LES simulations. Due to computa- 
tional expense, the former finds usage comparing a number of parametric geometries, while the latter becomes useful to more fully understand flow structures present in a single geometry. 
Table 2.1: This table shows a survey of parameters studied in literature. All rib cross sections are square and rib pattern is transverse unless otherwise indicated. Some parameter specifications are complex and are left as 'various'.

\begin{tabular}{|c|c|c|c|c|c|c|c|}
\hline Author & Year & $\mathrm{Re}$ & $e / D_{h}$ & $p / e$ & $W / H$ & Rib type & Methods \\
\hline [34] & 1992 & $10 \mathrm{k}-60 \mathrm{k}$ & $0.047,0.078$ & 10 & $0.25,0.5,1,2,4$ & $30,45,60,90^{\circ}$ & HT,PD \\
\hline [55] & 1994 & $10 \mathrm{k}-60 \mathrm{k}$ & $0.133,0.165,0.25$ & $5,7,8.5,10$ & 1 & $90^{\circ}$ staggered & HT,PD \\
\hline [40] & 1996 & $30 \mathrm{k}$ & 0.1 & $6-16$ & 1 & $90^{\circ}$ & LDV,PD \\
\hline [9] & 1997 & $10 \mathrm{k}-80 \mathrm{k}$ & 0.0625 & 8 & 1 & $90^{\circ}$ & HT,PD \\
\hline [54] & 1998 & $10 \mathrm{k}-25 \mathrm{k}$ & $0.133,0.167,0.25$ & $5,8.5,10$ & 1 & $90^{\circ} \frac{2}{3} \mathrm{AR}$,staggered & HT \\
\hline [42] & 2001 & $30 \mathrm{k}$ & 0.1 & 9 & 1 & $90^{\circ}$ & RANS \\
\hline [53] & 2002 & $30 \mathrm{k}$ & 0.125 & 10 & 1, 2-pass & $90^{\circ}$ & PIV \\
\hline [10] & 2002 & $50 \mathrm{k}$ & 0.1 & 10 & 1, 2-pass & $45^{\circ}$ staggered & PIV \\
\hline [1] & 2003 & $20 \mathrm{k}, 47.3 \mathrm{k}$ & 0.1 & 10 & 1 & $45^{\circ}$ staggered, $90^{\circ}$ & LES \\
\hline [4] & 2003 & $20 \mathrm{k}-100 \mathrm{k}$ & $0.193,0.333$ & 10 & 0.4 & $45^{\circ}$ staggered & TLC \\
\hline [60] & 2004 & $10 \mathrm{k}-40 \mathrm{k}$ & 0.078 & 10 & 4, 2-pass & $45^{\circ} \mathrm{V}, \mathrm{W}$, broken $\mathrm{V}, \mathrm{W}$ & $\mathrm{PD}, \mathrm{HT}$ \\
\hline [51] & 2005 & $20 \mathrm{k}$ & 0.1 & 10 & 1, 2-pass & $90^{\circ}$ & LDV,LES \\
\hline [17] & 2006 & $10 \mathrm{k}$ & Various & 10 & $0.25,0.5,1,2,4$ & $45^{\circ}$ & RANS \\
\hline [43] & 2007 & $30 \mathrm{k}$ & 0.1 & 9 & $0.25,1,4$ & $90^{\circ}$ & RANS \\
\hline [39] & 2009 & $30 \mathrm{k}-400 \mathrm{k}$ & $0.1,0.18$ & $5-10$ & 1 & $45^{\circ}$ & HT,PD \\
\hline [8] & 2011 & $2.5 \mathrm{k}-20 \mathrm{k}$ & 0.12 & 8 & 1 & $90^{\circ}$ staggered & PIV \\
\hline [57] & 2011 & $10 \mathrm{k}-40 \mathrm{k}$ & $0.1,0.4$ & 6.11 & 2 & wedges & HT,PD \\
\hline [61] & 2013 & $12 \mathrm{k}-60 \mathrm{k}$ & 0.0875 & 20 & 1 & $90^{\circ}$ truncated & RANS \\
\hline [26] & 2013 & $40 \mathrm{k}$ & 0.3 & 10 & 1 & $90^{\circ}$ & PIV,LES \\
\hline [24] & 2013 & $90 \mathrm{k}-450 \mathrm{k}$ & $0.02,0.03,0.06$ & 10 & $2,4,8$ & $\mathrm{~W}, 2 \mathrm{~W}, 4 \mathrm{~W}$ & TLC,RANS \\
\hline [30] & 2014 & $100 \mathrm{k}-1.3 \mathrm{M}$ & Various & Various & 6 & $45^{\circ} \mathrm{w} /$ dimples & HT \\
\hline [2] & 2015 & $6 \mathrm{k}-180 \mathrm{k}$ & 0.063 & 10 & 1 & $45^{\circ}$ & $\mathrm{PD}, \mathrm{HT}$ \\
\hline [33] & 2015 & $48 \mathrm{k}-183 \mathrm{k}$ & 0.078 & 10 & 4 & $45^{\circ}$ & RANS \\
\hline [52] & 2016 & $19.5 \mathrm{k}-69 \mathrm{k}$ & 0.125 & 16 & 1, 2-pass & $45^{\circ} \mathrm{V}, \mathrm{W}, \mathrm{M}$ & RANS,PD, TLC \\
\hline [23] & 2016 & $24 \mathrm{k}$ & 0.0625 & $2-18$ & circular & $90^{\circ}$ & LES \\
\hline [3] & 2016 & $61.4 \mathrm{k}-69.4 \mathrm{k}$ & 0.125 & single rib & 4 & $90^{\circ}$ trapezoid, various angles & PIV,TLC \\
\hline [28] & 2017 & $5 \mathrm{k}-20 \mathrm{k}$ & 0.1 & 10 & 1, parallelogram & $90^{\circ}$ & PIV \\
\hline
\end{tabular}




\subsection{Review of DMD Literature}

In the current era of relatively cheap and powerful parallel computing, analysis of large datasets have become more popular. Just as IDC geometries range far and wide, so do algorithms designed to extract patterns and trends from enormous datasets, which may source from experimentation or computational methods. One aspect of this wide field involves the extraction of spatial or transient spectral shapes present via algorithms of different types. This research focuses on the use of Dynamic Mode Decomposition (DMD), proposed by Schmidt in [48], which attempts to find a prediction of the future state of a system via a modal decomposition with little to no knowledge of the underlying system's behavior. The decomposition acts only on measurements of the system in time to produce a 'best fit' between the measurements. DMD can be applied not only to fluid systems, but also to video processing, controllers, and other dynamical systems, Kutz et al. [25]. The DMD process can be executed in real-time provided the system under measurement is simple enough.

DMD has some similarity to the older Proper Orthogonal Decomposition (POD) method, first introduced by Lumley [7], which extracts orthogonal spatial modes of a system. The two methods share some characteristics, and as much study with POD has been performed, researching it was warranted.

POD extracts the "most energetic" orthogonal modes of the system. This ranking may or may not well represent transient structures inside of a flow, leading to the usefulness of DMD. Its modes are generally not time invariant. It has found use in various fields related to fluid mechanics in heat transfer. Much like DMD, cavities and cylinders have been analyzed, as in Hossain et al [19], and Zhang et al. [62]. Typical outputs include visualizations of modal structures present in the fluid, which often exhibit themselves in the transient sense as coherent structures. Zhang et al in [62] also builds a comparison of POD and DMD upon a fabricated pattern, in an attempt to show the power of these methods on explicitly enforced fields. Even with just a few modes, the original fields 
(which are rather simple) can be reconstructed with good accuracy. POD has also found use in heat transfer and aerodynamics specific geometries, many with relation to gas turbines. Mahapatra et al. in [29] performs an analysis of natural convection in an enclosure, showing the dominance of the first several modes and some dependence of modal energy based on Rayleigh number. Park et al. [35] found usage on an oscillating jet, showing the energy related to fluctuations inside the jet core. Saha et al. [44] discussed modal analysis of a vortex generator. It was determined that for a turbulent flow, a large number of modes may be necessary for good field resolution; a good corollary to the turbulent spectrum. It was also shown that turbulent structures can be extracted from the modes. From a system perspective, POD has also been used to size heat exchangers on power plants, filling in detail where simulations were not performed, as in $\mathrm{Hu}$ et al. [20]. POD is not unknown to IDC-type geometries, as shown by Tyagi et al. [56]. They performed an analysis of a 90 degree rib turbulator, again showing many modes may be required to capture the energy of a turbulent flow. Coherent structures with the highest energy were related to impingement on the rib front surface and flow separation/attachment. POD was also used in conjunction with PIV on a rotating two-pass channel by Coletti et al. in [11]. His analysis suggested fluctuation of the separation bubble aft of a rib may be mostly controlled by the rib pitch. Of extraordinary relevance to this research is work performed by Schwanen et al. [50], where POD identifies modes important to heat transfer in a pin-fin geometry on a gas turbine blade. By identifying a dominate heat transfer mode, the geometry was modified to enhance this mode, showing a plausible increase in heat transfer.

DMD has seen more limited use compared to POD due to its relative youth. Unlike POD, DMD is time invariant, and modes are not necessarily orthogonal. In addition, its mode ranking does not depend based on an "energy" value, but rather on the dynamics of the system, which better accommodates some flows. Schmid introduced the concept in [48]. He applied it to helium jets in [47], a lid driven cavity in [46], transitional flat-plate flow in [45] and proposed a "sparsity promoting" version in [21]. The algorithm can be used on more industrial cases as well: Muld et al. 
[31] performed a comparison of DMD and POD in extracting coherent structures in the wake of a high-speed train. The idea of using certain modes in conjunction with the mean mode is also used to better understand flow structures. Li et al. showed a decomposition of a heated flapping fin in [27]. Rowley et al. showed application of the DMD method on a jet in a crossflow in [41]. Kalghatgi and Acharya showed a DMD analysis on an inclined cooling jet in [22]. DMD was also applied to a gas turbine oscillating jet in Wen et al. [58] and to a film cooling jet in Wenwu et al. [59]. The latter applies the DMD analysis directly to an engineering quantity rather than a primitive transport variable, in that case a heat transfer effectiveness field. This is a similar-sounding concept to the one proposed here. An application on a combustor was shown in Grenga et al. [16], which gives thorough review on DMD convergence metrics, often missing in many papers. Much of the POD and DMD research to date involves identification of structures and their associated spectral qualities. However there is little research showing a direct relation between a mode and an engineering quantity of interest (heat transfer, in this case). Furthermore, using this relation to enhance a quantity of interest, to the author's knowledge, has only been performed with a POD algorithm in [50] upon a pin fin.

With exhaustive research performed parametrically on the design space of IDC features in gas turbines, DMD may offer a methodology to seek data-driven design of rib turbulators with topologies which are spatially complex. Seeking such a methodology would be a powerful tool for the engineer, and is the subject herein. 


\section{CHAPTER 3: METHODOLOGY}

\subsection{Physics Introduction}

This section reviews important physics parameters utilized by this research.

\subsubsection{Pressure Loss/Friction Factor}

Pressure drop for internal flows is often characterized non-dimensionally by use of the friction factor, $f$. Its definition is shown in equation 3.1. It should be noted in this case the Darcy friction factor is utilized, which is four times the also commonly-used Fanning friction factor.

$$
f=\frac{d p}{d x} \frac{D_{h}}{\frac{1}{2} \rho u^{2}}
$$

The friction factor often forms the enemy of turbulent heat transfer. Turbulence promotes mixing, but often requires more power to push flow compared to a less-turbulent flow. Therefore one must always balance the heat transfer performed with the pressure required to drive the flow. There are some geometries which have excellent heat transfer properties, but are too demanding to drive flow through them. The engineer searches for an optimal configuration of high heat transfer for a low pressure loss or friction factor. 


\subsubsection{Heat Transfer Coefficient}

A dimensional number which characterizes the ability of a surface to transfer heat is known as the heat transfer coefficient, or HTC. This is defined in equation 3.2.

$$
h=\frac{Q}{A_{h}\left(T_{b}-T_{w}\right)}
$$

The HTC can also be non-dimensionalized as the Nusselt number, shown in equation 3.3.

$$
N u=\frac{h D_{h}}{k_{f}}
$$

To compare to other works, the Nusselt number ratio is often used. This is merely the Nusselt number above normalized by the result of the Dittus-Boelter value, as shown in equation 3.4.

$$
\frac{N u}{N u_{0}}=\frac{N u}{0.023 R e_{D_{h}}^{\frac{4}{5}} P r^{0.4}}
$$

Evaluation of the Nusselt number or HTC is peformed by assessing the heat flux at the wall and utilizing an energy balance in the flow direction to derive the bulk temperature. For internal flows with contant-temperature walls, the bulk temperature rises logarithmically, eventually meeting the wall temperature provided that the duct is long enough. After that point, very little heat transfer occurs, as the temperature is nearly constant in every location. The heat flux at the wall can be evaluated via the conduction law:

$$
q_{w}=k_{f} \frac{T_{w}-T}{\Delta s}
$$

with fluid thermal conductivity $k_{f}$, first-gridpoint spacing $\Delta s$, and local temperature $T$. This is only valid for extremely small $\Delta s$, within the linear sublayer. 


\subsection{CFD Model Introduction}

\subsection{Geometry}

The case at hand involves the geometry shown in figure 3.1. The geometric features are identical to an experimental setup in [18]. Its geometric parameters are shown in table 3.1. However this geometry only contains 3 ribs to reduce the computational expenses. The leading edge of the first rib is $0.05 \mathrm{~m}$ from the inlet, about $1 D_{h}$. The trailing edge of the last rib is $0.13 \mathrm{~m}$ from the outlet, about 2.5 $D_{h}$. This geometry will be changed slightly for the second RANS case, after the DMD analysis.

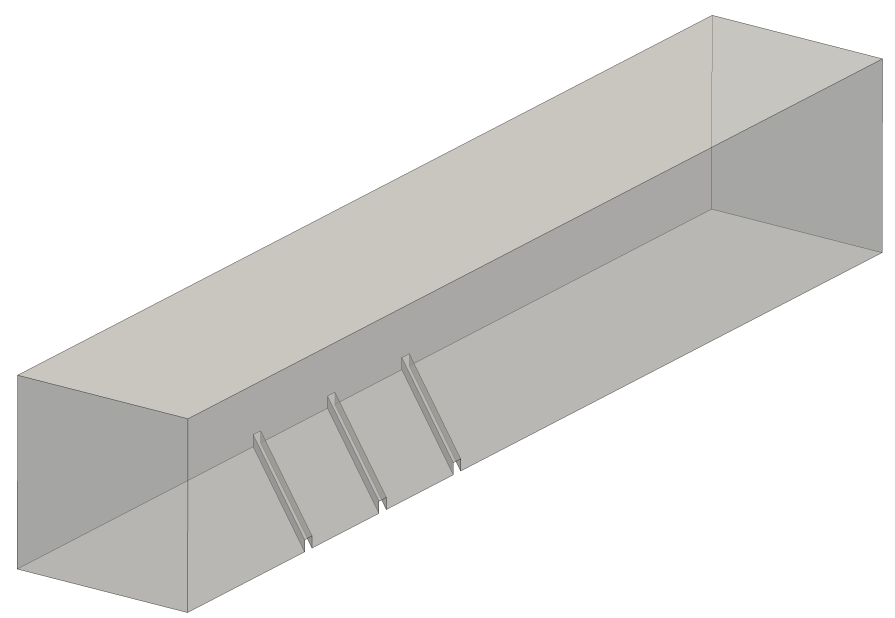

Figure 3.1: RANS baseline and LES case geometry 
Table 3.1: Geometric parameters for this study

\begin{tabular}{cll} 
Parameter & Value & Unit \\
\hline$D_{h}$ & 0.0508 & $\mathrm{~m}$ \\
$\alpha$ & 45 & degrees \\
$e / D_{h}$ & 0.063 & - \\
$p / e$ & 10 & - \\
$W / H$ & 1.0 & -
\end{tabular}

\subsection{Mesh Generation}

A high-quality mesh is required for any CFD simulation. This is especially important for LES, where the use of less-diffusive discretization schemes is desired. While relatively diffusive upwind schemes are useful for RANS simulations, when high-fidelity frequency content is desired, central differencing provides a less diffusive and 2 nd order accurate option. The geometry in use here is simple enough to allow the construction of a hexahedral mesh, providing excellent quality cells. The grid used for all cases is 32.5 million cells, and has ample resolution in the near-wall region, as will be discussed in later sections. Care is taken to ensure that no extreme volume growth rates exist; in general, all cells stay under a ratio of 1.2. Images of the mesh are available in figures 3.2 and 3.3. 


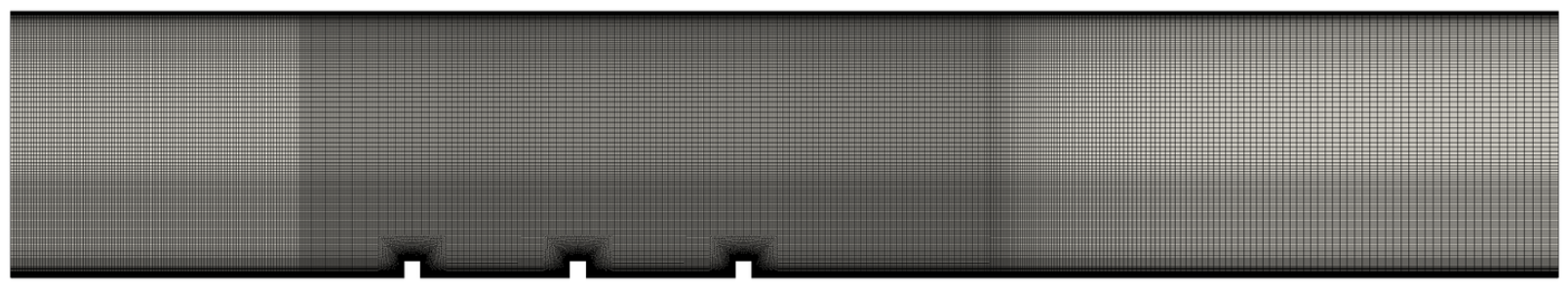

Figure 3.2: Center cut of computational mesh

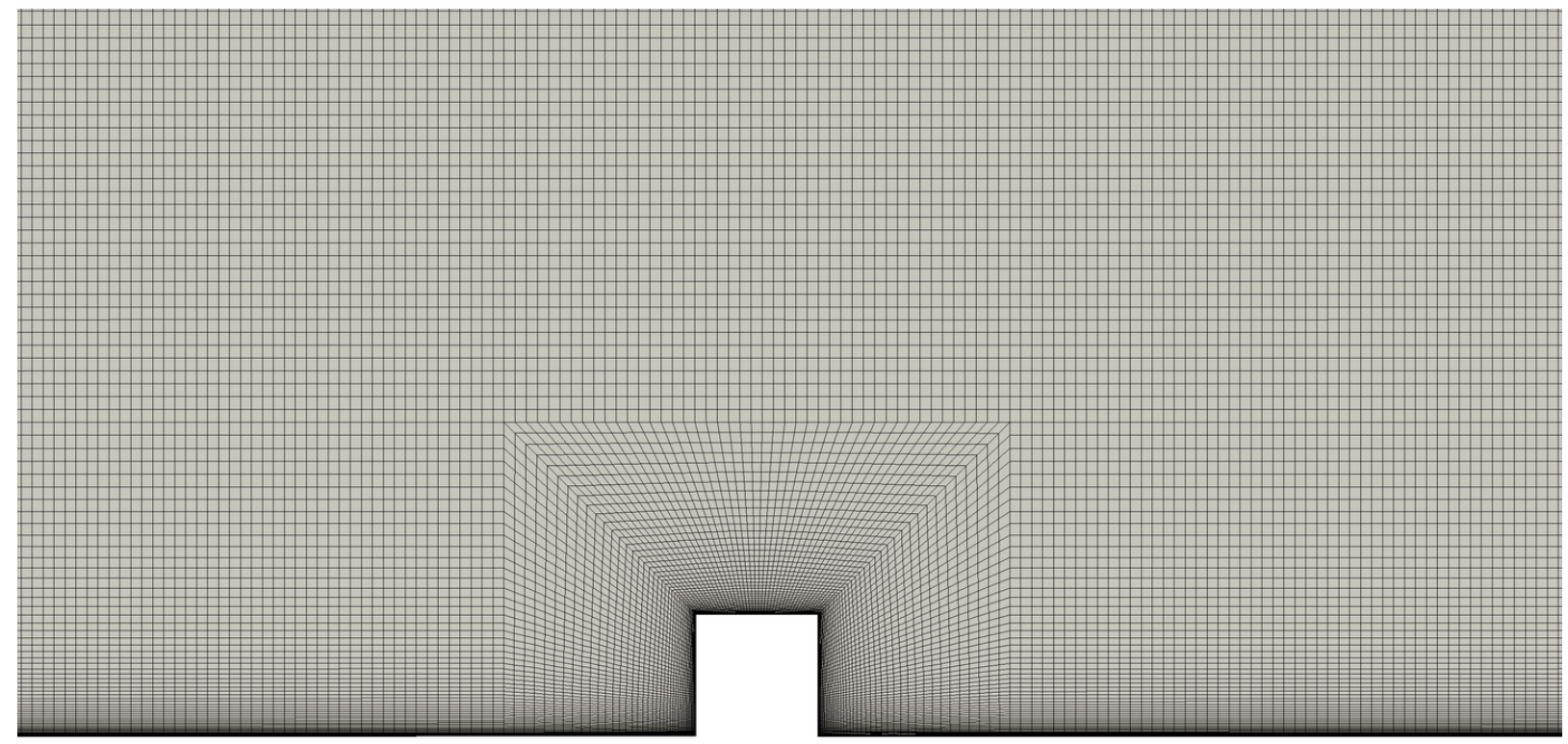

Figure 3.3: View of computational mesh around rib, center cut

\subsection{RANS Numerical Setup}

Steady RANS simulation have been an industry workhorse for many decades and allow a good tradeoff between computational accuracy and expense. Derivations of the RANS equations are covered exhaustively in literature and is not performed here, however the procedure is similar to that found in the SGS background section 3.6.2. The main issue with RANS modeling is the 
closure of the Reynolds stresses which arise due to the Reynolds averaging procedure. A number of turbulence models exist to close the equation system and recover some of the behavior of an unsteady turbulent flow without resolving the field in fine detail. In this case, the popular $k \omega-\mathrm{SST}$ model has been chosen, as it provides superior performance in wall-bounded and separated flows, which are of great interest here.

The RANS equations are solved with the finite-volume method and the equations are coupled using the SIMPLEC algorithm, the details of which can be found in [13]. The ideal-gas law is utilized as the equation of state for the medium, which is merely air.

\subsubsection{Discretization}

Convective terms in the RANS simulation are resolved using a second-order upwind scheme, which forms much of the industry standard for finite-volume CFD. The artificial dissipation effects of upwind schemes are well-known. A second-order upwind scheme utilizes an additional gridpoint upstream of a given cell, allowing it to have a much lower dissipative effect.

\subsubsection{Convergence Criterion}

The RANS simulations are run until the residuals from each transport equation drop at least 3 orders of magnitude. In addition, the heat transfer from the rib surfaces are all monitored with every iteration, and they must not change appreciably with iteration to call the run converged.

\subsubsection{Near-Wall Resolution}

As a grid identical to the LES simulation is used for RANS cases, and the LES case has more restrictive requirements, no changes are made to the near-wall mesh for RANS specifically. The 
LES near-wall requirements are discussed later in section 3.6.5. The numerical wall treatment for RANS is capable of integration into the viscous sublayer, $y^{+}<1$.

\subsection{LES Numerical Setup}

Modal decomposition in time requires good resolution of the transient and spatial characteristics of the turbulence, for which RANS is too diffusive. Large Eddy Simulation (LES) is used to resolve the major flow structures in the domain, whose transient responses can be fed into the DMD algorithm. The LES simulation directly simulates the Navier-Stokes equations with the addition of a sub-grid scale (SGS) model, to account for eddies smaller than the grid can resolve. This filtering is done implicitly on the grid scale, which allows the SGS to activate in places where the mesh is too coarse to resolve all of the dynamics. The SGS chosen was the WALE model, which has shown good results in a variety of fields.

\subsubsection{Discretization}

The LES simulation is performed with a constant timestep of $1 \times 10^{-6}$ s using the second-order Crank-Nicolson scheme. The timestep is chosen such that the maximum CFL obeys equation 3.6.

$$
C F L=\frac{u \Delta t}{\Delta x} \leq 1
$$

While not strictly necessary for the stability of the (implicit) time discretization, other schemes present require a relatively low CFL for stability. The SIMPLEC algorithm is still utilized, as was on the RANS cases, which performs a number of inner-timestep iterations to converge the fields to an acceptable tolerance, the residual. A drop of 3 orders of magnitude relative to the first inner iteration is defined as converged. Once this criterion is reached, inner iteration halts and the 
timestep advances.

\subsubsection{Subgrid Scale Model}

To introduce the issues related to SGS modeling, we begin with the derivation of the residual stress tensor. The incompressible Navier-Stokes equations are represented in equation 3.7.

$$
\frac{\partial \rho u_{j}}{\partial t}+\frac{\partial \rho u_{i} u_{j}}{\partial x_{i}}=\mu \frac{\partial^{2} u_{j}}{\partial x_{i} \partial x_{i}}-\frac{\partial p}{\partial x_{j}}
$$

As we are only interested in "large" eddies, a filter $G$ is applied to the velocity field, producing the filtered or resolved velocity $\bar{u}$ :

$$
\bar{u}\left(x_{i}, t\right)=\int G\left(x_{i}, x_{i}^{\prime}, \Delta\right) u\left(x_{i}^{\prime}, t\right) d x_{i}^{\prime}
$$

In this case the filter is of the typical top-hat type and the cutoff length scale $\Delta$ is computed with the cube root of the volume of the cell. Upon filtering, we produce:

$$
\frac{\partial \rho \bar{u}_{j}}{\partial t}+\frac{\partial \rho \overline{u_{i} u_{j}}}{\partial x_{i}}=\mu \frac{\partial^{2} \bar{u}_{j}}{\partial x_{i} \partial x_{i}}-\frac{\partial \bar{p}}{\partial x_{j}}
$$

We note that $\overline{u_{i} u_{j}} \neq \bar{u}_{i} \bar{u}_{j}$, so we are left with an unclosed system, as only the filtered velocities are available from the computation. However, we can say:

$$
\frac{\partial \overline{u_{i} u_{j}}}{\partial x_{i}}=\frac{\partial \bar{u}_{i} \bar{u}_{j}}{\partial x_{i}}+\left(\frac{\partial \overline{u_{i} u_{j}}}{\partial x_{i}}-\frac{\partial \bar{u}_{i} \bar{u}_{j}}{\partial x_{i}}\right)
$$

Allowing the components of the final term combined with the density to be re-named the residualstress tensor:

$$
\tau_{i j}=\rho\left(\overline{u_{i} u_{j}}-\bar{u}_{i} \bar{u}_{j}\right)
$$


We can then form the LES equations:

$$
\frac{\partial \rho \bar{u}_{j}}{\partial t}+\frac{\partial \rho \bar{u}_{i} \bar{u}_{j}}{\partial x_{i}}=\mu \frac{\partial^{2} \bar{u}_{j}}{\partial x_{i} \partial x_{i}}-\frac{\partial \bar{p}}{\partial x_{j}}-\frac{\partial \tau_{i j}}{\partial x_{i}}
$$

There is a notable similarity to the RANS equations present. Now we require some model for the final term on the right-hand side. A reasonable approach is the common eddy-viscosity $\left(\mu_{t}\right)$ assumption:

$$
\tau_{i j}=\mu_{t} \bar{S}_{i j}
$$

with the resolved deformation tensor $\bar{S}_{i j}$ as:

$$
\bar{S}_{i j}=\frac{1}{2}\left(\frac{\partial \bar{u}_{i}}{\partial x_{j}}+\frac{\partial \bar{u}_{j}}{\partial x_{i}}\right)
$$

For this analysis we adopt the Wall-Adapting Local Eddy-viscosity (WALE) model. The computation of the turbulent viscosity as reported in [32] is:

$$
\mu_{t}=\rho C_{w}^{2} \Delta^{2} \frac{\left(S_{i j}^{d} S_{i j}^{d}\right)^{\frac{3}{2}}}{\left(\bar{S}_{i j} \bar{S}_{i j}\right)^{\frac{5}{2}}+\left(S_{i j}^{d} S_{i j}^{d}\right)^{\frac{5}{4}}}
$$

where $C_{w}$ is a model constant, and $S_{i j}^{d}$ is the traceless symmetric part of the velocity gradient tensor squared. In short, this model features a number of advantages over the typical Smagorinsky model. These advantages include (1) proper scaling of the turbulent viscosity close to the wall without usage of a damping function or dynamic computation of variables, (2) accounts for both strain and rotation from the given fields, and (3) is general enough to apply on complex geometries, without need for ambiguous flow direction or wall distance specifications. Though different Smagorinksy models have been proposed through time to match these advantages, the WALE model is used here. 


\subsubsection{Evaluating Statistical Stationarity}

The LES simulation is carried out until statistical convergence, which is determined by utilizing a reverse-arrangements test [6] on numerous probe data from the simulated domain. Probe points are placed along the centerline of the domain, as well as in a grid behind the last rib, which should be the slowest to develop. These points can be seen in figure 3.4.

The reverse arrangements test is simple in construction and execution. The null hypothesis states that the observations are independent, while the alternative supposes the observations have an underlying trend. The test is executed on $N$ observations of a variable $x_{i}=\left\{x_{1}, x_{2}, x_{3}, \ldots, x_{N}\right\} . x_{i}$ is then compared with every following observation $x_{j}$. If $x_{i}$ is larger than the following observation, an integer $h_{i j}$ is incremented by 1 :

$$
h_{i j}= \begin{cases}1 & x_{i}>x_{j} \\ 0 & x_{i} \leq x_{j}\end{cases}
$$

Once all of the integers of $x_{i}$ have been summed, $i, j$ are incremented and the integers are added again until all observations have been iterated through. This generates a final number of reversals, $A_{r}$ :

$$
A_{r}=\sum_{i=1}^{N-1} \sum_{j=i+1}^{N} h_{i j}
$$

$A_{r}$ has a mean value:

$$
\mu_{A_{r}}=\frac{N(N-1)}{4}
$$

and variance:

$$
{\sigma_{A_{r}}}^{2}=\frac{N(2 N+5)(N-1)}{72}
$$

The number of reversals can be compared to what a random distribution should carry within a 95\% confidence interval for the same $N$. This can be done based on a lookup table[6], or by computing 
the value $z$, given in [5]:

$$
z=\frac{A_{r}-\mu_{A_{r}}}{\sigma_{A_{r}}}
$$

The null hypothesis is rejected at the $95 \%$ level for $-1.96 \geq z \geq 1.96$, indicating for this range a significant trend in the observations.

Figure 3.4: Sampling probe locations for statistics

\subsubsection{Grid Resolution}

A turbulent flow is often characterized as a cascade of 'scales', where large turbulent eddies contain a wealth of energy. Dissipation causes these eddies to break up and collapse into smaller eddies. It is theorized that the breakup of eddies continues until the eddies become so small, viscous forces damp them completely and dissipate their energy as heat. More information on the turbulent scales is presented in section 3.6.6. Due to the large range in scales, resolution of each scale completely is computationally impractical - hence the "large" in LES. Pope [38] proposes that resolving 80\% of the energy with a computational mesh is a sufficient tradeoff to directly resolving every eddy. This mesh is designed to meet such a goal. In most literature, this is evaluated by computing the Turbulent Kinetic Energy (TKE) resolution ratio. The resolved TKE is defined as equation 3.20.

$$
k_{r e s}=\frac{1}{2}\left(\overline{u^{\prime} u^{\prime}}+\overline{v^{\prime} v^{\prime}}+\overline{w^{\prime} w^{\prime}}\right)
$$


Where $u^{\prime}$ is the fluctuation velocity, computed from the Reynolds decomposition, $u^{\prime}=u-\bar{u}$. In this case the a value with a line $\left(\overline{u^{\prime} u^{\prime}}\right)$ indicates the temporal average. The unresolved TKE is computed from the SGS model as $k_{s g s}$. Then the TKE ratio can be defined as:

$$
\bar{k}_{\text {ratio }}=\frac{\bar{k}_{\text {res }}}{\bar{k}_{\text {sgs }}+\bar{k}_{\text {res }}} \geq 0.8
$$

In the LES case presented here, this criterion is obeyed by $99.7 \%$ of cells in the domain. Closer to $100 \%$ would be preferable, and further work would include improvement of this metric. Additional information on alternative metrics are provided in Appendix C.

\subsubsection{Near-Wall Resolution}

As the features of interest in this flow exist on the walls and incorporate heat transfer, the resolution of the boundary layer is of highest importance. The first grid point in this case is placed inside the laminar sublayer such that $y^{+}<1$. For these cases, $y^{+}<0.6$ is true the vast majority of the time. With RANS, there is no general requirement on the mesh in the other dimensions tangent to the wall. However, in LES, it is important structures close to the wall are captured. Since these structures are very anisotropic in nature, grid spacing tangent to the wall is not as demanding as the wall-normal size, and thus it is often required the tangential spacing be as low as 30 viscous units in $z^{+}$and $x^{+}$. Though many different values are available in literature, some being as high as 100 [37] [36]. In this case, $x^{+}$and $z^{+}$are constrained to be less than 30 . The value varies greatly over the surface and often averages around 15. In general, any viscous unit $s^{+}$with grid spacing $\Delta s$ can be defined:

$$
s^{+}=\frac{u_{f} \Delta s}{\nu}
$$


where $u_{f}$ is the friction velocity, calculated from density and wall shear $\sqrt{\frac{\tau_{w}}{\rho}}$, and $\nu$ is the kinematic viscosity. Figure A.1 shows the three wall spacing values for the LES case. The RANS cases are not shown but since they use an identical mesh, they do not differ greatly.

\subsubsection{Turbulent Scales}

Turbulent flows are often characterized by a cascade of eddies which range in size from large to small. No eddy can be as large as the geometric scale, $\mathcal{L}_{0}$, which is a characteristic size based on the geometry at hand. Here the hydraulic diameter, $D_{h}$ is chosen. The largest eddies are considered to be $1 / 3$ of this geometric scale. We refer to this new scale as $\mathcal{L}=\frac{1}{3} \mathcal{L}_{0}$. Many theories of turbulence involve larger eddies dissipating their energy into smaller scales in a recursive cascade until the eddies become so small that the viscosity of the fluid dissipates them as heat. This final scale is termed the Kolmogorov scale, and can be computed: $\kappa=\mathcal{L} R e^{-\frac{3}{4}}$. In between these two scales are a range of intermediate scales. These are often termed the "energy containing" or inertial scales. At these scales dissipative effects of viscosity are not yet felt; energy merely passes from one scale to the next with no net heat generation. Often the largest inertial scale is approximately $I_{L}=\frac{1}{6} \mathcal{L}$. The smallest, after which dissipative effects start to occur, is approximately $I_{S}=10 \kappa$.

For LES, we desire to resolve a number of these scales and leave the remaining to be modeled via the SGS. This allows a significant savings in computational time. In an effort to appreciate this time savings, one can calculate the requirement for a Direct Numerical Simulation (DNS), resolving all scales to the Kolmogorov scale explicitly. Knowing the scale size, timestep in use, the CFL condition, and the iteration time per timestep per cell of the computational resources used for the LES case, we can estimate the runtime for a DNS case. This estimate is presented in table 3.2. Note that 'core' refers to a single computing core, which may reside inside of a multi-core CPU (assumed to be x86-64). Even with significant increases in computational resources (assuming linear parallel scaling), the DNS run is not feasible for engineering purposes. The runtime for RANS 
and LES cases of this study are also logged in appendix D.

There are many who criticize the (sometimes) gross assumptions needed to compute fluid flow numerically. This calculation shows the appreciation all should have to the efforts of countless scientists and engineers whom have worked to overcome seemingly insurmountable obstacles provided by physics and lived to speak of the knowledge they garnered.

Table 3.2: Estimated DNS run metrics

\begin{tabular}{crr} 
Parameter & Value & Unit \\
\hline Cell edge length & $2.25 \times 10^{-5}$ & $\mathrm{~m}$ \\
Number of cells & $66.7 \times 10^{6}$ & - \\
Core hours for run & $5.9 \times 10^{8}$ & core*hr \\
Runtime with current resources & $2.1 \times 10^{6}$ & $\mathrm{hr}$ \\
Runtime with current resources & 242 & years
\end{tabular}

\subsection{Boundary Conditions}

For RANS cases, the inlet is treated as a constant velocity, $10 \mathrm{~m} / \mathrm{s}$, and temperature, $T_{i}$ condition. A pressure (0 gauge) is specified at the outlet. All other surfaces are walls with a specified constant temperature $T_{w}$. The turbulence wall treatment allows integration of the primitive variables to the viscous sublayer. These boundary conditions are summarized in table 3.3. Gradient operators indicate the gradient normal to the wall.

Table 3.3: RANS cases boundary conditions

\begin{tabular}{cccccc} 
Boundary & Velocity & Pressure & Temperature & TKE & SDR \\
\hline Inlet & Constant $10 \mathrm{~m} / \mathrm{s}$ & $\nabla p=0$ & $T_{i}=300 K$ & $1 \%$ intensity & Constant \\
Outlet & $\nabla \mathbf{U}=0$ & $p=0$ gauge & $\nabla T=0$ & $\nabla k=0$ & $\nabla \omega=0$ \\
Walls & No-slip & $\nabla p=0$ & $T_{w}=351.15 K$ & low-Re & low-Re
\end{tabular}

For LES cases, the inlet maintains its constant-velocity condition, but is modified to allow random 
fluctuations of the constant velocity, $10 \mathrm{~m} / \mathrm{s}$, by as much as $3 \%$. In addition, the LES cases have a constant-pressure outlet with a convective velocity outlet condition. This convective outlet, instead of a usual zero face-normal gradient, imposes equation 3.23 on the boundary. $U$ is a specified velocity needed to maintain the desired mass flow. This condition allows pressure waves to leave the domain, preventing noise-generating reflections [13].

$$
\frac{\partial \phi}{\partial t}+U \frac{\partial \phi}{\partial n}=0
$$

In addition, a damping zone near the outlet is specified to prevent the possibility of backflow occurring and creating further disturbances of the fields, which LES modeling can be sensitive to. The pressure loss incurred by the damping zone is not included in any pressure drop or friction factor calculation. Its approximate location is noted by the purple dashed line in figure 3.5. The LES boundary conditions are summarized in table 3.4. Gradient operators indicate the gradient normal to the wall.

Table 3.4: LES case boundary conditions

\begin{tabular}{cccc} 
Boundary & Velocity & Pressure & Temperature \\
\hline Inlet & Constant $10 \mathrm{~m} / \mathrm{s}$ with random noise & $\nabla p=0$ & $T_{i}=300 \mathrm{~K}$ \\
Outlet & Convective & $p=0$ gauge & $\nabla T=0$ \\
Walls & No-slip & $\nabla p=0$ & $T_{w}=351.15 K$
\end{tabular}




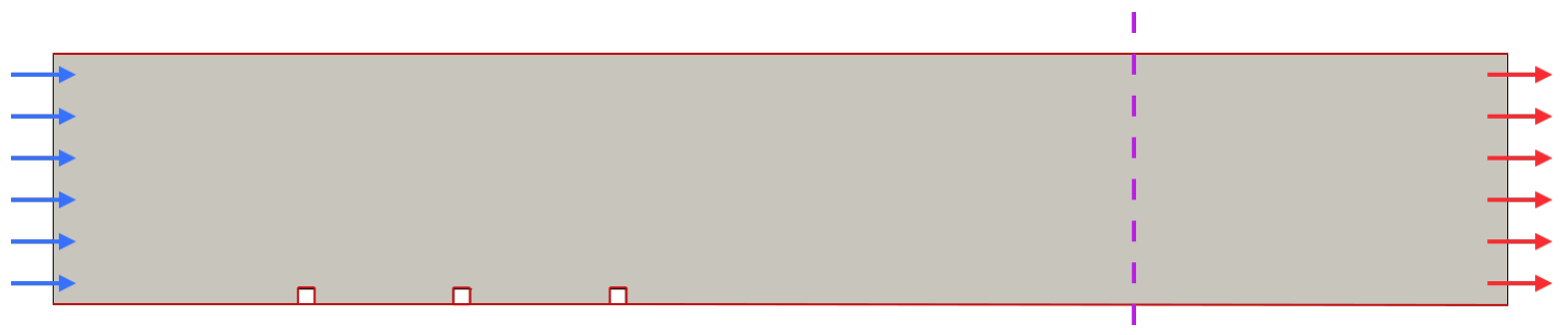

Figure 3.5: Diagram of boundary conditions. Heated walls are noted in dark red. The purple dashed line shows the approximate location of the outlet damping zone.

\subsection{DMD Introduction}

Dynamic Mode Decomposition (DMD) is an algorithm that was introduced by Schmidt in [48]. The algorithm produces approximations to a dynamic system's eigenvalues and eigenvectors. These approximations can be used to not only reproduce the system at a previously observed time state, but also to predict future time states with good accuracy. The DMD scheme employed here uses the "method of snapshots", where the input data result from measurement of the variables of interest. These measurements occur on a series of probe points, generated around each rib. Each point probe 'volume' contains 666070 points each. An example of a coarse probe grid can be found in figure 3.6, and a visualization of the volume they occupy is in figure 3.7. The method of snapshots allows the DMD algorithm to apply identically to numerical data from a CFD code or from highfidelity experimental data like PIV. Acquiring data from the computational grid directly would be preferred to a probe grid, but since the solver in use treats all cells in an unstructured manner, this is complex to implement. Numerical schemes used here are also second-order, requiring a rather fine mesh compared to higher-order methods. This would increase the costs of DMD were the computational grid to be used. Furthermore, a DMD code which operates from measured data can easily find equal use on experimental datasets, aiding future studies. 
These snapshots are important to understanding the goal of DMD. Suppose that measurements of a system are taken at $n$ points for $m$ timesteps. These measurements are gathered into two matrices, $X_{1}$ and $X_{2}$ :

$$
\begin{aligned}
& X_{1}=\left\{x_{1}, x_{2}, x_{3}, \ldots, x_{m-1}\right\} \\
& X_{2}=\left\{x_{2}, x_{3}, x_{4}, \ldots, x_{m}\right\}
\end{aligned}
$$

This means that each point in time $x_{j}$ contains $n$ measurements of the system, all at different positions, resulting in two $n \times(m-1)$ matrices. Suppose now that the system operates in a linear manner such that the transformation matrix $A$ exists:

$$
X_{2}=A X_{1}
$$

$A$ itself is the best fit used to take, for example, $x_{2}$ to $x_{3}$ while simultaneously taking $x_{6}$ to $x_{7}$. DMD attempts to produce an approximation to this matrix which keeps enough detail to resolve important dynamics and ignores enough detail to be computationally efficient. Several steps are used to determine important content the system and produce a new, approximate $A^{\prime}$. This approximation is good enough to make future predictions of the system state in time for a short period. It will soon be shown actually attempting to produce the real $A$ creates the need for extremely large and expensive computers, whereas $A^{\prime}$ is cheap enough to run on a conventional workstation or less for many systems.

Those new to DMD should not be intimidated, as much of its theory revolves around eigenvalue solutions many engineers are comfortable with. Recall that our desired system in equation 3.26 is a linear combination solution to the differential equation

$$
\frac{d \mathbf{x}}{d t}=\mathbf{A} \mathbf{x}
$$


The solution to this equation can also be represented as

$$
x(t)=\sum_{k=1}^{n} \phi_{k} e^{\lambda_{k} t} b_{k}
$$

where $\phi_{k}$ is the $k$ th eigenvector and $\lambda_{k}$ is the $k$ th eigenvalue of $A . b_{k}$ is an initial condition. In a simplistic view, DMD just gives an easy way to approximate the eigenvalues and eigenvectors, which will allow us to create an good reconstruction of $x$ at any $t$.

Since the DMD process seeks a linear transformation between time states, it obviously assumes a linear fit to the system being modeled. For a statistically stationary flow, this is likely a decent approximation. Note however that, while the end map is linear in nature, the method of generating the map comes from the measurement of a nonlinear system. That is, the DMD need not be performed on a linearized set of equations, so the underlying input dynamics will contain some of that information inherently. Since we only rely on snapshots of the system, the DMD method here essentially becomes "equationless" modeling, and allows flexible usage on fields far outside fluid mechanics. The assumption of linearity can be too strong for some fields, but on many timescales and for engineering purposes it is a good enough assumption. An amusing poem by an unknown author states:

The world is non-linear, it's true.

But fear not, for here is a clue:

When signals are small, and stabl' overall, $\dot{x}=A x$ will do.

The DMD algorithm can be quite inexpensive with modern linear algebra packages. The code developed here utilizes the Python libraries NumPy, SciPy, and sparsesvd. Vectorized code and sparse matrix datastructures make for efficient computations, even with thousands of retained timesteps on millions of gridpoints, without need for a distributed memory computer. The sparsesvd package 
implements the SVDLIBC library, which is generally quite robust even on very sparse matrices. However there exits another useful SVD library which implements the SVD provided by ARPACK. It was found ARPACK was generally faster, but showed poor robustness for sparse matrices. Runtime metrics for DMD cases performed here can be found in Appendix D.

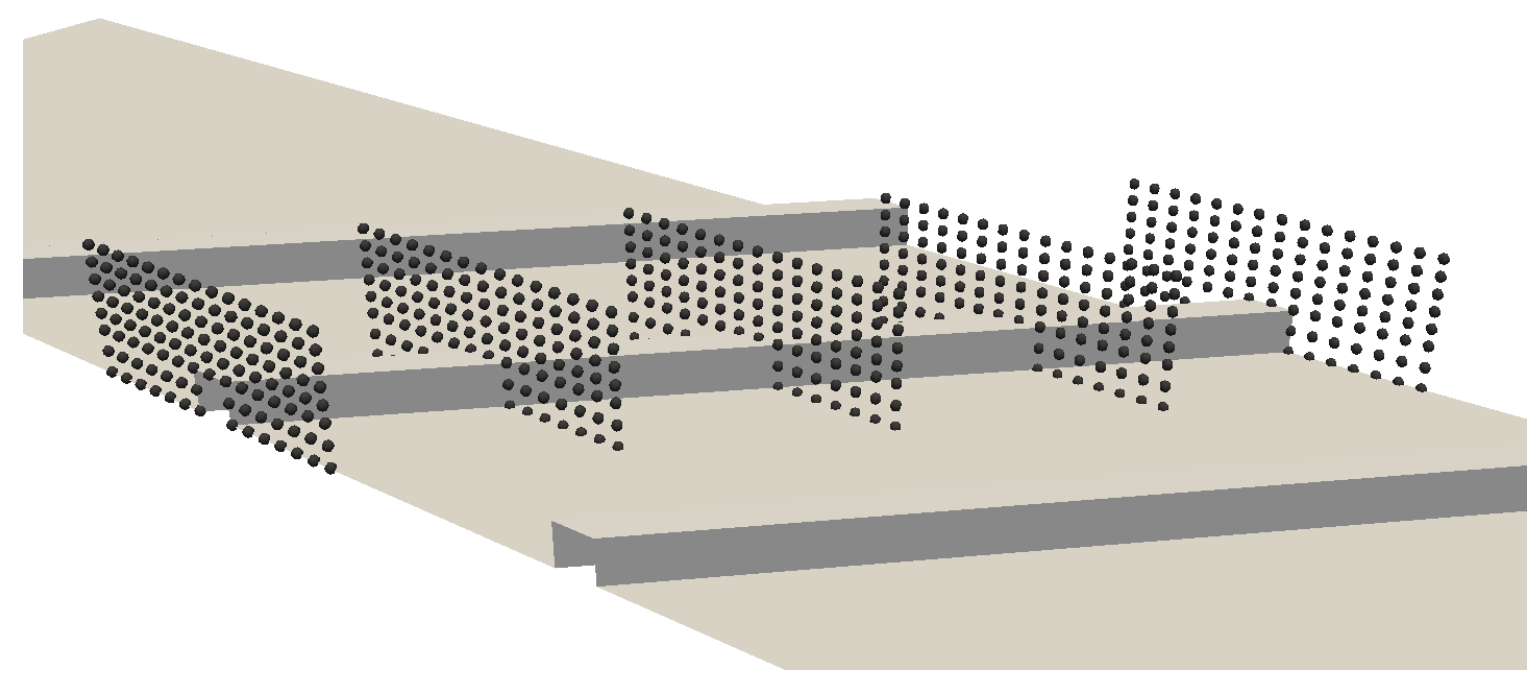

Figure 3.6: Example point probe grid, forming the DMD volume. The grid used for analysis is much finer with about 700k probes. 


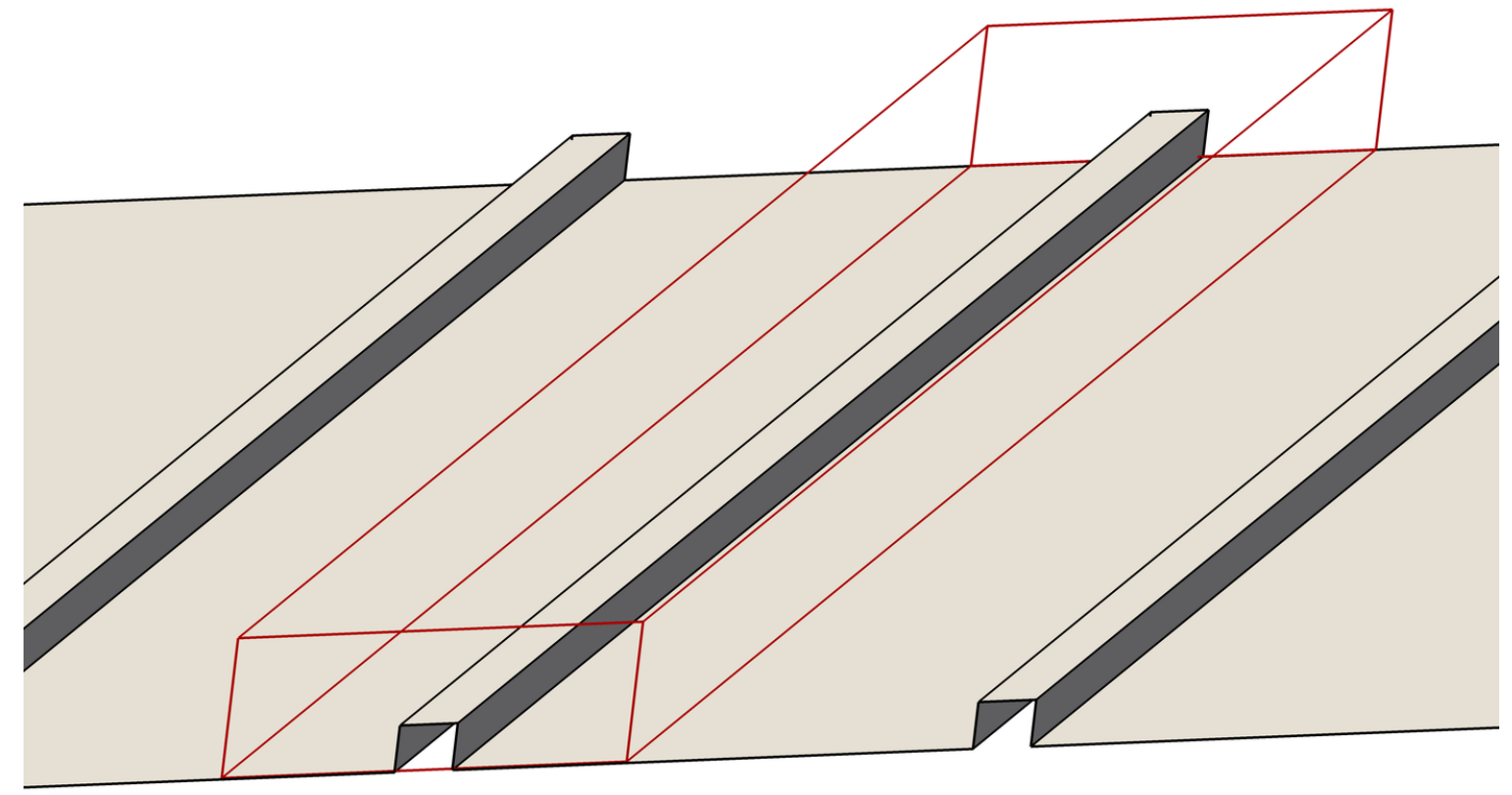

Figure 3.7: Outline of DMD domain. The red lines indicate the 'volume' which is used for DMD.

\subsubsection{DMD Algorithm Process}

The DMD algorithm starts with a series of snapshots separated by uniform timesteps across a region of space. Recall the goal of the DMD algorithm is to produce an approximation $A$ between two datasets $X_{1}$ and $X_{2}$ such that $X_{2} \approx A X_{1}$. The DMD algorithm utilizing the method of snapshots begins with the snapshots themselves. Some experiment or numerical method is run to produce the quantity of interest over $n$ points for $m$ timesteps. One timestep $m$ with all spatial data is denoted as $x_{m}$.

$$
X=\left\{x_{1}, x_{2}, x_{3}, \ldots, x_{m}\right\}
$$


We split $X$ to form two data sequences. The time interval then between these sequences is exactly one timestep, $\Delta t$.

$$
\begin{aligned}
& X_{1}=\left\{x_{1}, x_{2}, x_{3}, \ldots, x_{m-1}\right\} \\
& X_{2}=\left\{x_{2}, x_{3}, x_{4}, \ldots, x_{m}\right\}
\end{aligned}
$$

As stated in the introduction, $A$ is the best single matrix which advances each measurement in $X_{1}$ in time one $\Delta t$. It would be possible in theory to calculate $A$ doing nothing but the inverse of $X_{1}$ :

$$
A=X_{2} X^{+}{ }_{1}
$$

$X_{1}^{+}$is the Moore-Penrose pseudoinverse of $X_{1}$. This calculation is often termed "exact" DMD. However since $X_{1}$ is a large dimensional matrix $(n \mathrm{x} m)$, generation of $A$ is costly or impossible, especially on shared-memory computers.

To overcome this obstacle, we utilize the singular value decomposition (SVD). This factors $X$ into three matrices (equation 3.33). $U$ are the left-singular vectors of $X$ and correspond to the POD modes, orthogonal in space [7]. It is $n \times n$. $\Sigma$ are the singular values of $X$, and is $n \times m$. The singular values are often interpreted as the "energy" values of the corresponding POD modes; this is exploited later. $V^{*}$ are the right-singular vectors of $X$, and is $m \times m$. The symbol * indicates the complex conjugate of the transpose of the given matrix.

$$
U \Sigma V^{*}=X_{1}
$$

$A$ could now be computed:

$$
A=X_{2} V \Sigma^{-1} U^{*}
$$


Also note that if Principle Component Analysis (PCA) is desired, one need only to subtract the mean $\bar{X}$ from $X$ and perform the SVD. The POD modes would then correspond to a PCA analysis output.

The resulting $A$ is $n \times n$; still impractical. We wish to approximate $A$, not attain it in completeness, however. Therefore a rank-reduction is performed to eliminate singular values beyond some threshold. For now, say the singular values are truncated at rank $r$. This can be interpreted as keeping only the most energetic POD modes. Then, $U$ becomes $n \times r, \Sigma$ becomes $r \times r$, and $V$ becomes $r \times m$. Most of the detail in $A$ is contained in the first spatial modes, so this allows us to dramatically lower the computational cost of the algorithm without great sacrifice in fidelity. Using the SVD, it is then possible to compute a lower-dimensional $A^{\prime}$, which approximates $A$. We then project $A$ on to the (now low dimensional) POD modes.

$$
A^{\prime}=U^{*} A U
$$

However this can also be carried out more efficiently by using equation 3.34 :

$$
A^{\prime}=U^{*} X_{2} V \Sigma^{-1}
$$

We can then perform an eigendecomposition:

$$
A^{\prime} W_{v}=W_{v} \Lambda
$$

Where $W_{v}$ and $\Lambda$ are the eigenvectors and eigenvalues, respectively, of $A^{\prime}$. Now that we have a low-dimensional eigendecomposition (we can see $A^{\prime}$ is now $r \times r$ ) of the system, we need to go back to the higher-dimensional state for reconstruction of the solution. The DMD modes $\Phi$ in 
high-dimensional space can then be computed:

$$
\Phi=X_{2} V \Sigma^{-1} W_{v}
$$

Reconstruction of the data $x(t)$ using the $r$ DMD modes $\phi_{r}$, can be performed in the familiar manner using equation 3.39. Note that $t$ is not the physical time, but rather the time after the physical time from the initial condition, $x_{1}$.

$$
x(t)=\sum_{k=0}^{r} \phi_{k} e^{\omega_{k} t} b_{k}
$$

where $\omega_{k}=\ln (\lambda) / \Delta t$ for a timestep $\Delta t . b_{k}$ surmounts to an initial condition column of a given mode, which can be computed for $t=0$ :

$$
b_{0}=\Phi_{0}^{+} x_{1}
$$

$\Phi^{+}$is the Moore-Penrose pseudoinverse of $\Phi$. Equation 3.39 is extremely convenient, since we can plug in any desired time and get the approximated system at that time quickly and easily.

\subsubsection{Rank Truncation Methodology}

One consequence of removing some of the singular values from the SVD is that one must choose how many to retain. Often in literature it is proposed that, having plotted the singular values, a 'knee' will present itself, as in figure 3.8. After the 'knee', the value of retaining extra values to the solution diminishes greatly for each new value. This presents an obvious, if qualitative, method for choosing the number of ranks to retain. Further analysis on keeping additional ranks is given in section 3.9 .3 


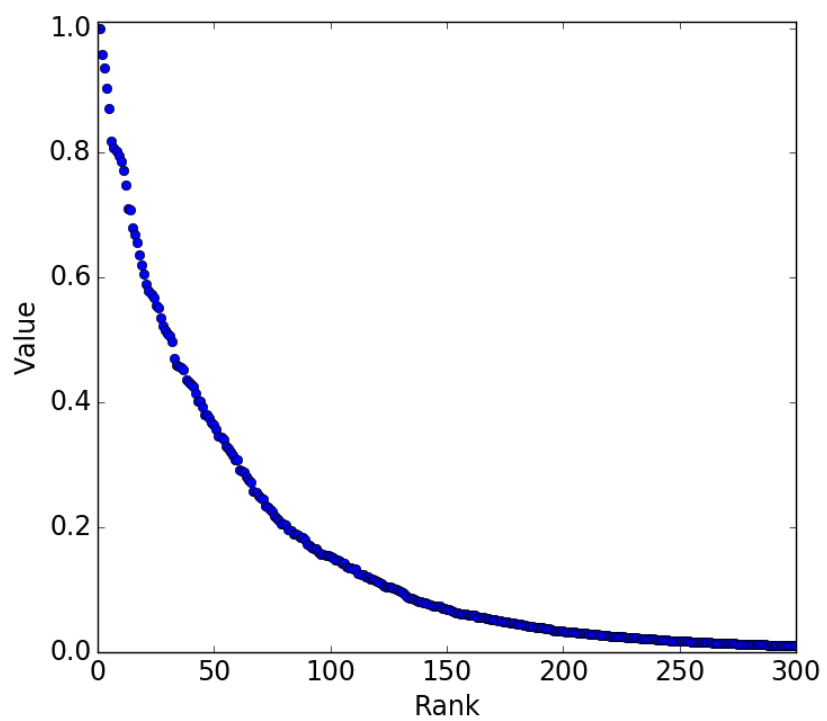

Figure 3.8: Example of normalized singular values, displaying a 'knee'.

\subsubsection{DMD Mode Amplitude and Frequency}

From the quantities developed in section 3.8.1, there are also other important definitions which are available. In particular are the amplitudes of the DMD modes, as well as their frequencies. As stated, the DMD explores data in a similar manner to the Fourier transform in the temporal sense. As such, the DMD modes can have a similar power evaluation, where an amplitude of each mode can be used to assess its relative contribution to the spectrum. The DMD amplitude can be computed as

$$
A_{\Phi}=\left|\Phi^{+} \mathbf{b}\right|
$$

where $\mathbf{b}$ is the column of initial conditions from equation 3.39.

The DMD algorithm runs through several routines which are a part of different pre-compiled Python packages. This means that the modes do not necessarily appear in the same order from run to run with slightly different parameters. To alleviate this, the DMD modes are sorted accord- 
ing to their amplitude in a decreasing fashion. Mode 0, corresponding to the average, will have the highest amplitude. In this manner, the order in which the modes appear is deterministic. For POD analyses, this is often unnecessary, as many SVD libraries already order the modes in terms of their energy, calculated from their singular values. Ordering the SVD modes is convenient for methods which involve truncating the series at a certain rank.

It is sometimes helpful to think of equation 3.39 as a conglomerate of functions which depend on space and time, and the amplitude, which can be generally expressed:

$$
X(x, t)=\sum_{k=0}^{\infty} \phi_{k}(x) \Omega_{k}(t) A_{\Phi}(x)
$$

In addition, the frequencies these amplitudes occur at are readily computed from the eigenvalues:

$$
f(k)=\frac{\operatorname{Im}\left(\ln \left(\lambda_{k}\right)\right)}{2 \pi \Delta t}
$$

Where $\operatorname{Im}()$ returns the imaginary part of the argument.

\subsubsection{Modal Contribution to Engineering Quantities}

Of great interest is a general method of computing the relevance of a particular mode to a certain engineering quantity. In this case, heat transfer serves as the candidate, but a useful method would be applicable to any other quantity, such as drag or lift. The procedure here is shown using the example of the temperature field since heat transfer is of interest, but in general the procedure applies to any desired quantity.

Once the DMD modes are extracted following the procedure in section 3.8.1, a reconstructed temperature field $T_{r}(x, t)$ should be available. In this case, we are interested in a heat transfer value, so the time-average of the reconstructed flow field $\bar{T}_{r}(x)$ is calculated. The engineering 
quantity, heat transfer, is then evaluated on the field and compared to the output of the simulation. This is done via the Fourier conduction law and applied over the 'faces' of each point in the DMD point grid closest to the rib boundary. For this to apply, the first grid point must be very close to the wall, inside the viscous sublayer.

Next we compute reconstructed partial temperature fields for each set of modes $T_{p}(x, t, j)$ by selectively including certain modes. The first mode constitutes the average and generally performs a large amount of the heat transfer. The field for a given mode includes the contribution of itself and all modes previous to it. This means the final number of temperature fields is equal to the number of resolved modes, $r$.

$$
T_{p}(x, t, j)=\sum_{k=0}^{j} \phi_{k} e^{\omega_{k} t} b_{k}, j=1,2,3, . . r
$$

$\overline{T_{p}}(x, j)$ can then be used with the Fourier law to derive a new heat transfer value. For example, the first field $j=1$ would be the mean heat transfer field, as it includes only the first DMD mode, which corresponds to the mean. For $j=2$, the first and second modes would be used to create $\overline{T_{p}}(x, 2)$. Comparing $\overline{T_{p}}(x, 1)$ and $\overline{T_{p}}(x, 2)$ will show what effects, if any, the first mode has on the temperature field. For both of these examples, the heat transfer quantities result from the temperature field, thus each mode will have its own heat transfer quantities. Comparing them shows which modes contribute to heat transfer, which detract from it, and which perform nearly no function. This method is very general and can apply to nearly any quantity. The same code which calculates a given engineering quantity from an arbitrary primitive field $F$ can be used repeatedly for calculation of the same quantity on its partial fields, $F_{p}$, which makes the method easy to implement. 


\subsection{DMD Convergence Study}

The DMD algorithm presented includes several parameters which must be chosen. These are:

- Number of retained ranks $(m)$

- Time span of all snapshots, as a multiple of the DMD-volume flow-through time $(N)$

- Snapshot timestep $(\Delta t)$

A parameter implicit to these is the number of snapshots utilized, $n$. Some studies use this value instead of $N$, but it is the preference of the author to use the latter. These parameters can vary based on application and should be carefully studied. This section addresses assessing the convergence of the DMD modes with changes in each of these parameters.

\subsubsection{Parameter Limits}

Naturally first one turns to the bounds of the parameters, and any potential implicitness. It is obvious that $n>1$, but has no upper limit, as $N$ can be any positive integer. The timestep can be chosen to be as fast as the measurement time or slower, however results are subject to the Nyquist criterion in this analysis (but other algorithms may not have this requirement [25]). The number of retained ranks must also be larger than 1, however its upper bound is $m=n-1$, as any linear transform would require at least 2 time states. It can be limited artificially to save memory if desired, which occurs in this analysis. 


\subsubsection{Convergence Assessment Introduction}

The convergence assessment here takes a similar path to that of Schmid, [48], Grenga et al. [16], and Saha et al. [44]. The DMD is performed with differing parameters and the results are analyzed to determine when making those parameters larger gives diminishing returns on the resulting modes and reconstructed fields. Convergence of the algorithm is assessed with three main criterion: convergence of the eigenvalues to the unit circle, convergence of the modal amplitudes, and the convergence of the residual against the originally measured field. The eigenvalues take the name Ritz values, after the finite-element algorithm used to calculate them. The first assessment involves the tests in section 3.9.3, seeking understand the effects of increasing $m$. Following, an assessment using parameters in table 3.5 is conducted to determine a converged $N$ and $\Delta t$. This table also lists reference Strouhal numbers for convenience.

There are some important differences between this convergence assessment and others available in literature. The first is that [16] used the number of timesteps as a convergence variable, whereas here we use $N$. Using a constant total sampling time ensures each DMD run draws from the same pool of measurements, as opposed to increasing the amount of data available to consecutive DMD runs by increasing $n$. In addition, no explicit rank limit was applied, whereas here one exists, due to different code architectures. Another convergence assessment method was shown in [31]. There, the analysis assesses the change in each mode with orthogonality metrics. However this method suffers from several drawbacks when used with a wide number of modes. Because of these drawbacks, the method is not utilized.

Table 3.5 describes changes in $\Delta t$ as multiples of the lowest timestep of $20 \mu$ s and changes in $n$ as multiples of the DMD-grid flow-through time $N$, about $2.2 \mathrm{~ms}$ or 112 timesteps. This is the time it takes the average velocity to traverse the space occupied by the DMD probe grid. As an example, the value $N=5$ at $\Delta t=20 \mu s$ would refer to $n=560$. Also note that $N=10$ is about 1 flow-through time of the entire computational domain. 
Each entry in tables 3.5 represents an evaluation of the DMD algorithm $m$ times, where $m$ is either $n-1$ for $n<301$ or otherwise $m=301$. The limit of 301 modes is chosen to keep computational requirements at a reasonable level. Each evaluation of the algorithm stores the output metrics for increasing $m$, and allows determination of convergence. Once the desired parameters which produce good convergence are known, the process of computing modes for each $m$ from 1 to $n-1$ can be skipped in favor of running the algorithm once with the desired values, resulting in a substantial reduction in runtime. Details on runtime can be seen in appendix D.

Table 3.5: Number of timesteps $(n)$ in DMD convergence tests. A single flow through of the DMD sub-volume is 112 snapshots at the smallest timestep, seen for $N=1$ and $\Delta t=20 \mu \mathrm{s}$. The Strouhal number is defined using the inverse of the timestep, the rib height, and bulk velocity.

\begin{tabular}{|r|r|r|r|r|r|}
\hline & & \multicolumn{4}{|c|}{$N$ flow-throughs } \\
\hline $\mathrm{St}$ & $\Delta t[\mu \mathrm{s}]$ & 1 & 3 & 5 & 10 \\
\hline 15.63 & 20 & 112 & 336 & 560 & 1120 \\
7.81 & 40 & 56 & 168 & 280 & 560 \\
3.91 & 80 & 28 & 84 & 140 & 280 \\
1.95 & 160 & 14 & 42 & 70 & 140 \\
\hline
\end{tabular}

\subsubsection{Dependence on Number of Retained Ranks}

The option to retain only a number of singular values from the output of the SVD is compelling with the speed advantage it gives. To observe this parameter's effects on the rest of the previous convergence metrics, a study is performed which modifies the number of retained ranks $m$ for a constant $N$ and $\Delta t$. The number of retained ranks for each case are detailed in table 3.6.

Figure 3.9 shows the Ritz values for the associated tests on the real-imaginary plane. For a discrete time system, values inside of the unit circle would indicate dissipative modes, whereas those outside would represent explosive movies. The largest visible effect is perhaps the most expected one: 
Table 3.6: Number of retained ranks in DMD convergence tests

\begin{tabular}{|l|r|r|r|r|r|r|r|r|r|r|r|r|}
\hline Test & 1 & 2 & 3 & 4 & 5 & 6 & 7 & 8 & 9 & 10 & 11 & 12 \\
\hline Ranks $(m)$ & 27 & 51 & 77 & 101 & 127 & 151 & 177 & 201 & 227 & 251 & 277 & 301 \\
\hline
\end{tabular}

reducing the number of ranks reduces the maximum resolved frequency in the spectrum. Lowering the number of ranks is essentially a low pass filter on the system. Several of the higher values of $m$ have Ritz values slightly inside of the circle. This displays that keeping more ranks, while giving a greater portion of the spectrum, can introduce less well-converged modes. This can be viewed in figure 3.10 as well, which displays the spectrum of the tests. The noise of the high-rank datasets is easily visible against the seemingly clear low-rank datasets. Similar results were noted in [25]. It is important, then, to view the spectrum of any DMD output and ensure a low noise level.

Perhaps the most physically meaningful convergence check would be to compare the modal reconstructed field at the last timestep against the measured last timestep $\left(t=t_{f}\right)$ with a residual value, defined by:

$$
r_{i}=\frac{\sum_{k=0}^{r} \phi_{k} e^{\omega_{k}\left(t_{f}\right)} b_{k}-x\left(t_{f}\right)}{x\left(t_{f}\right)}
$$

The point-local residual $r_{i}$ is evaluated globally for all points $P$ by the sum:

$$
|r|^{2}=\left[\sum_{i=0}^{P} V_{i}\right]^{-1} \sum_{i=0}^{P} r_{i}^{2} V_{i}
$$

where $V_{i}$ is the volume surrounding an individual measurement point. A low value indicates a very good prediction of the measurement, and one would except the prediction to improve as more and more modes are utilized. Figure 3.11 exemplifies this, showing a residual becoming quite flat at around $m=151$. To further decrease the residual, the snapshot timestep must be reduced, as shown in later sections. The number of retained ranks (if chosen and not forced by the number of timesteps) should be chosen low enough to prevent excessive noise and high enough to give a 
satisfactory residual.

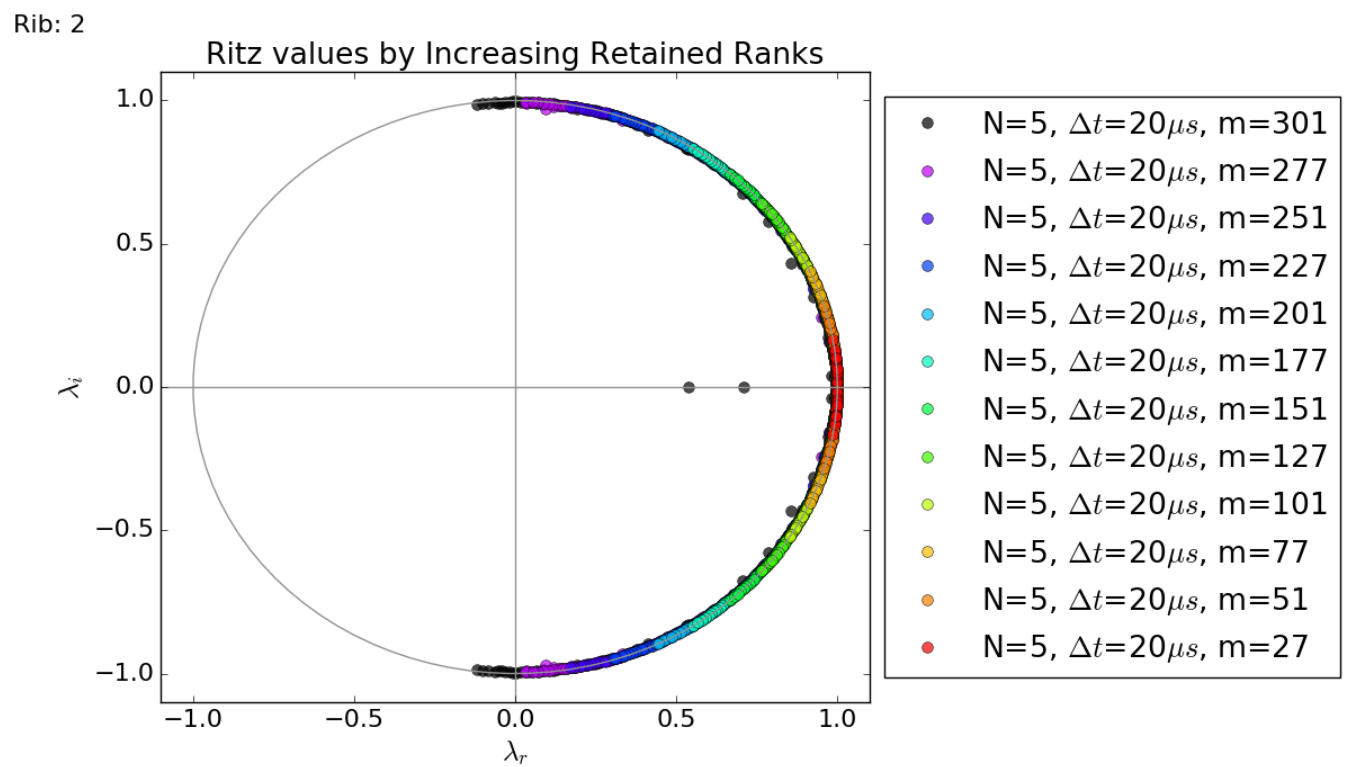

Figure 3.9: Ritz values for various retained ranks. 


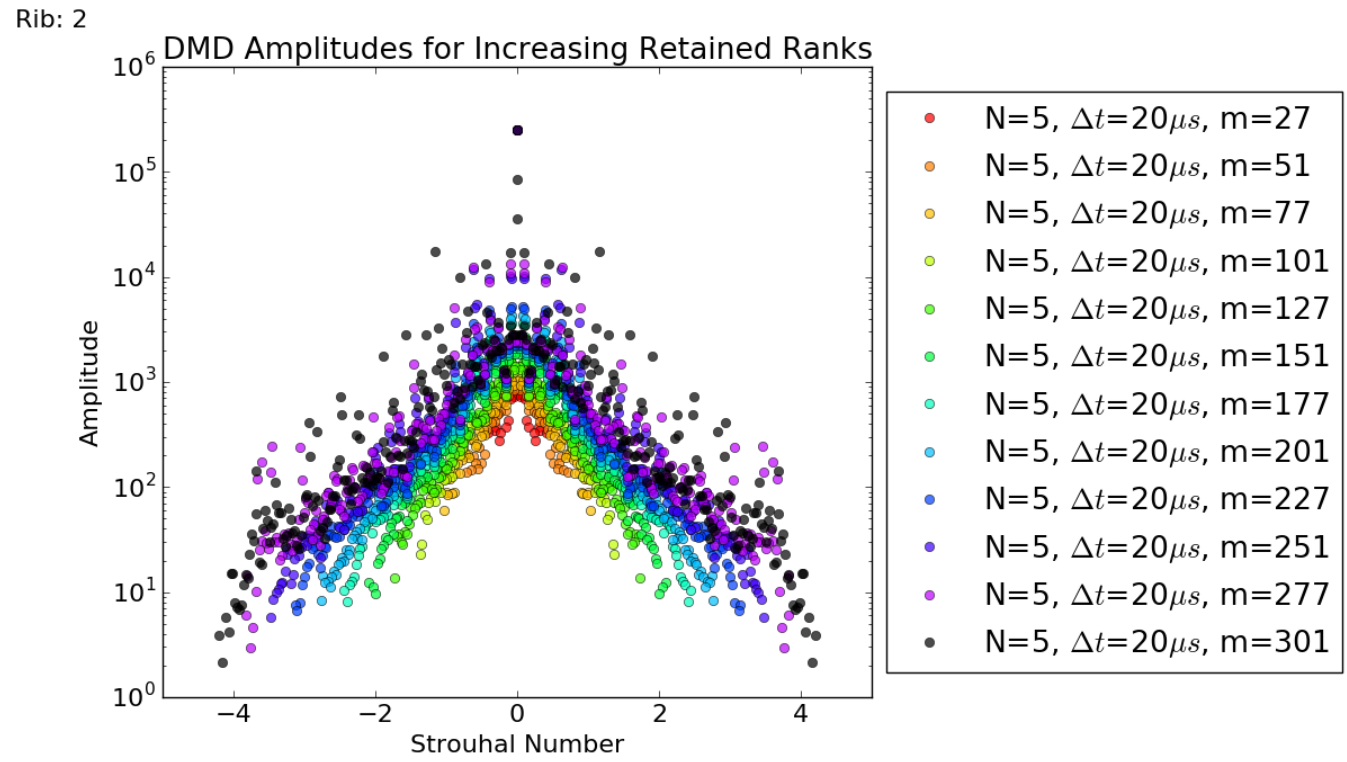

Figure 3.10: Spectral convergence for various retained ranks.

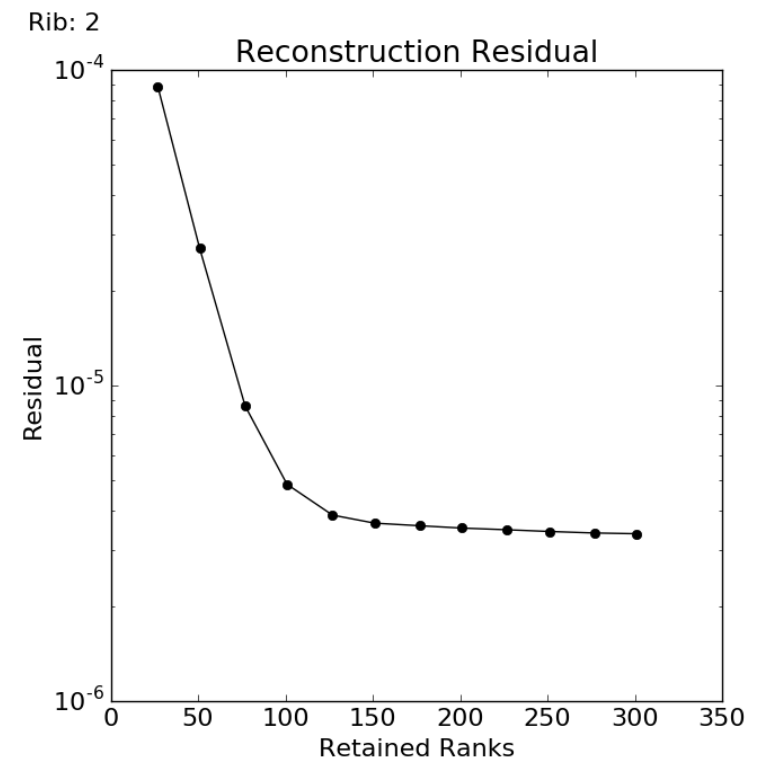

Figure 3.11: Residual convergence for various retained ranks. 


\subsubsection{Ritz Value Convergence}

The approximated eigenvalues, otherwise known as Ritz values, are a direct output of the DMD algorithm, and can be plotted on the real-imaginary plane for each run. Comparison of the columns and rows of table 3.5 are useful to determine changes in the system as a result of changes of a single input parameter.

Figure 3.12 shows the real and imaginary Ritz values for constant $N=1$, but increasing $\Delta t$, the first column in table 3.5. The number of retained modes, $m$, increases with decreasing $\Delta t$ since $N=1$ corresponds to $n<301$. As the timestep decreases, the maximum resolved frequency increases, but this is not evident from the figure; the values appear spread out and merely increase in (spectral) resolution. Furthermore, the values march towards the unit circle, indicating convergence, though no single test appears fully converged. The values lie inside the circle, corresponding to dissipative modes, and showing no converged datasets.

Figure 3.13 shows the analysis for the final column in table 3.5. Note that the two lowest timesteps have been limited to 301 modes, but only the lowest timestep succeeds in having all values close to the unit circle. However we can also see the lowest timestep has a reduction in spectral bandwidth, which seems counter-intuitive. Even though the timestep is smaller, the number of singular values is limited. A smaller timestep will still reveal additional low frequency modes with higher amplitudes, and the singular value truncation will low-pass filter away any higher frequencies attained, effectively reducing the bandwidth. This has the effect of lowering the noise relative to other datasets, and increasing the number of modes on the unit circle. The final dataset appears converged.

Figure 3.14 shows the analysis for the 3rd row in table 3.5. All $N$ appear with modes inside the circle, though generally increasing $N$ helps convergence.

Figure 3.15 shows the analysis for the 1 st row in table 3.5 . Only $N=10$ appears converged.

This set of figures reinforce the idea from [16] that the eigenvalue convergence is best viewed by 
changing both $N$ and $\Delta t$ together, as neither can bring about a converged result alone. However, one may question if a dataset appears not converged, which value brings about a greater change in the convergence towards the unit circle? This questions brings about an alternative way to plot these values which in the author's opinion is superior. Figure 3.16 shows a mapping of all the convergence cases together on a scale approaching a Ritz value magnitude of 1 . They are plotted against frequency merely for convenience. Clearly the low timestep, high $N$ cases are very close to 1 , indicating convergence. Cases are stratified with a combination of timestep and $N$, again implying that both need be chosen well in order to achieve a converged result. However it is easy to see that increasing $N$ (shapes) causes great leaps towards the magnitude 1, whereas decreasing $\Delta t$ (colors) is not as effective. This agrees with the results of [16], but is (perhaps) easier to identify. Certainly the tight range on the y-axis scale allows much greater definition on to the approach of the unit circle than viewing the entire unit circle in a single plot. One can conclude, then, if convergence to the unit circle is poor, increasing $N$ is more effective than decreasing $\Delta t$.

The remaining figures representing the other columns and rows in the convergence case table can be found in Appendix A. 


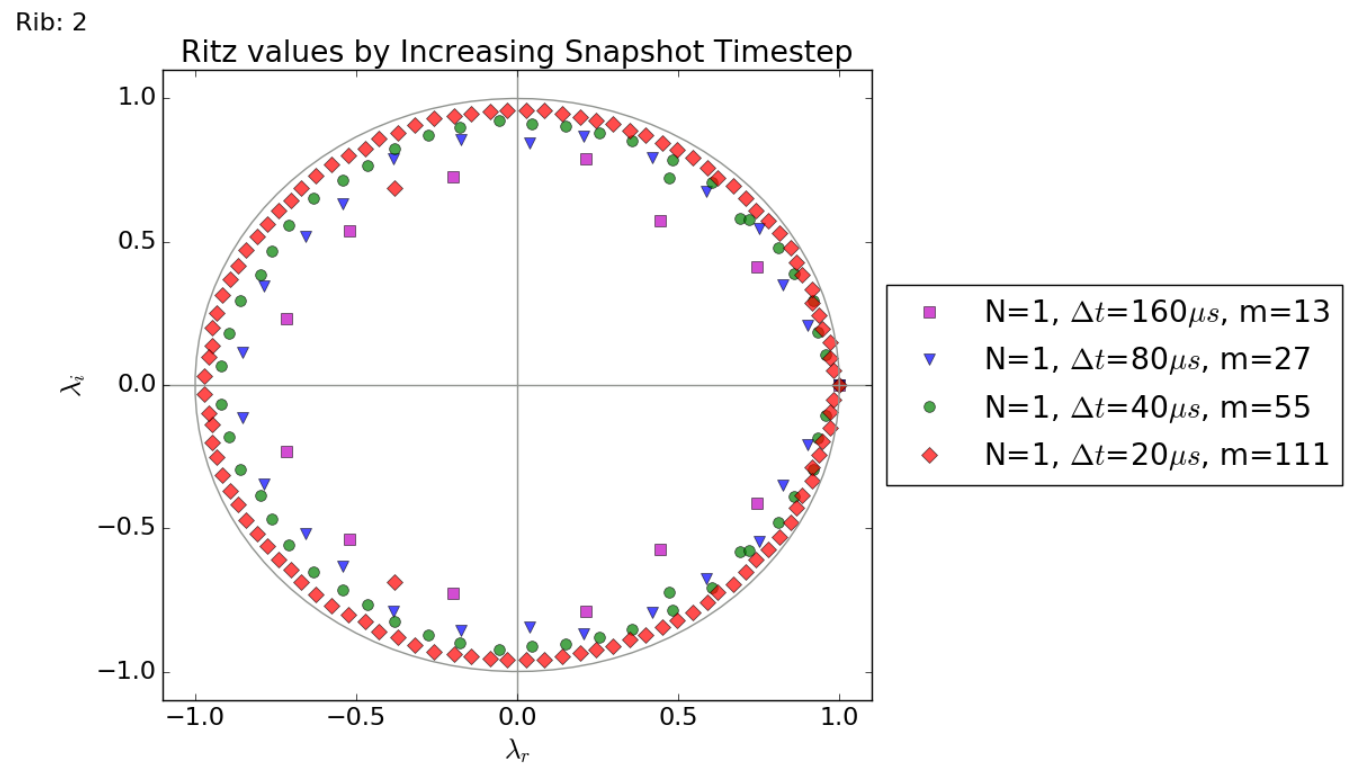

Figure 3.12: Ritz values for $N=1$.

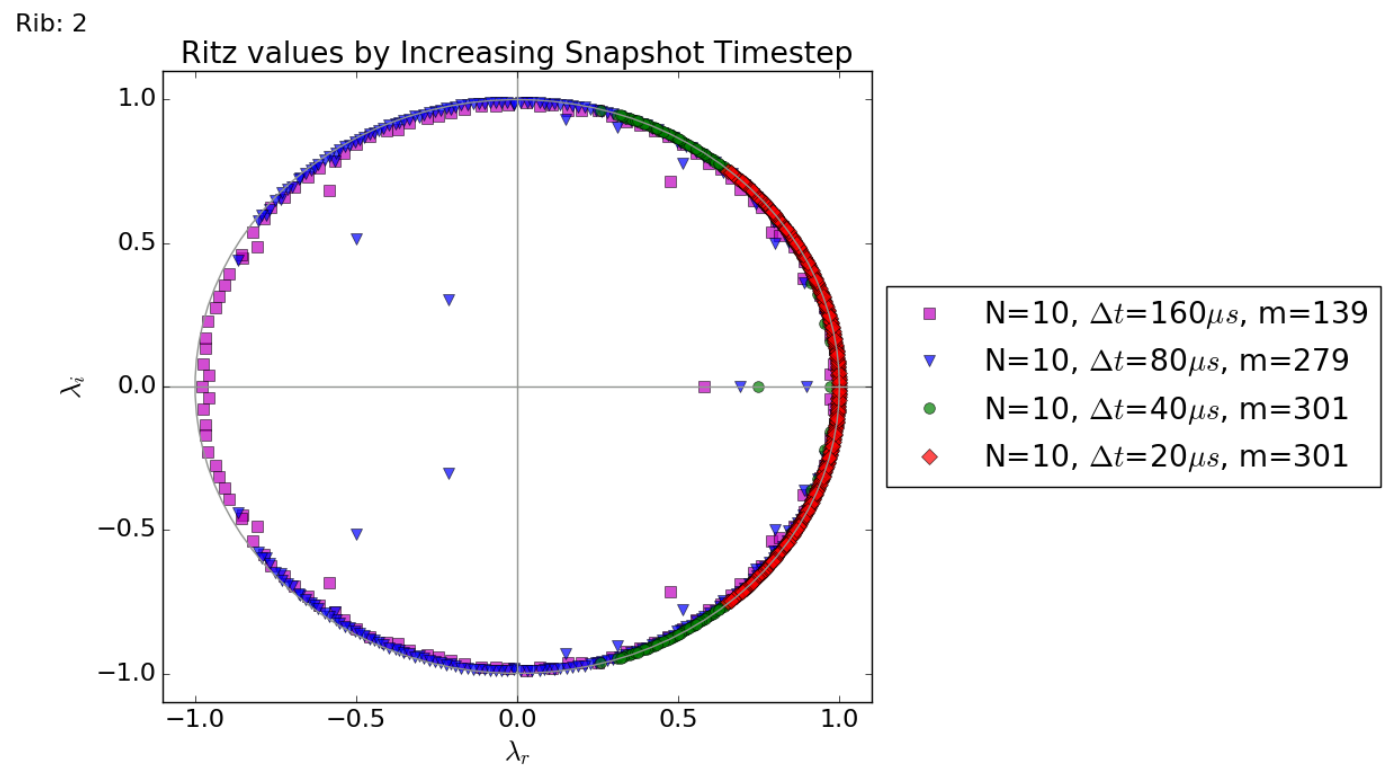

Figure 3.13: Ritz values for $N=10$. 


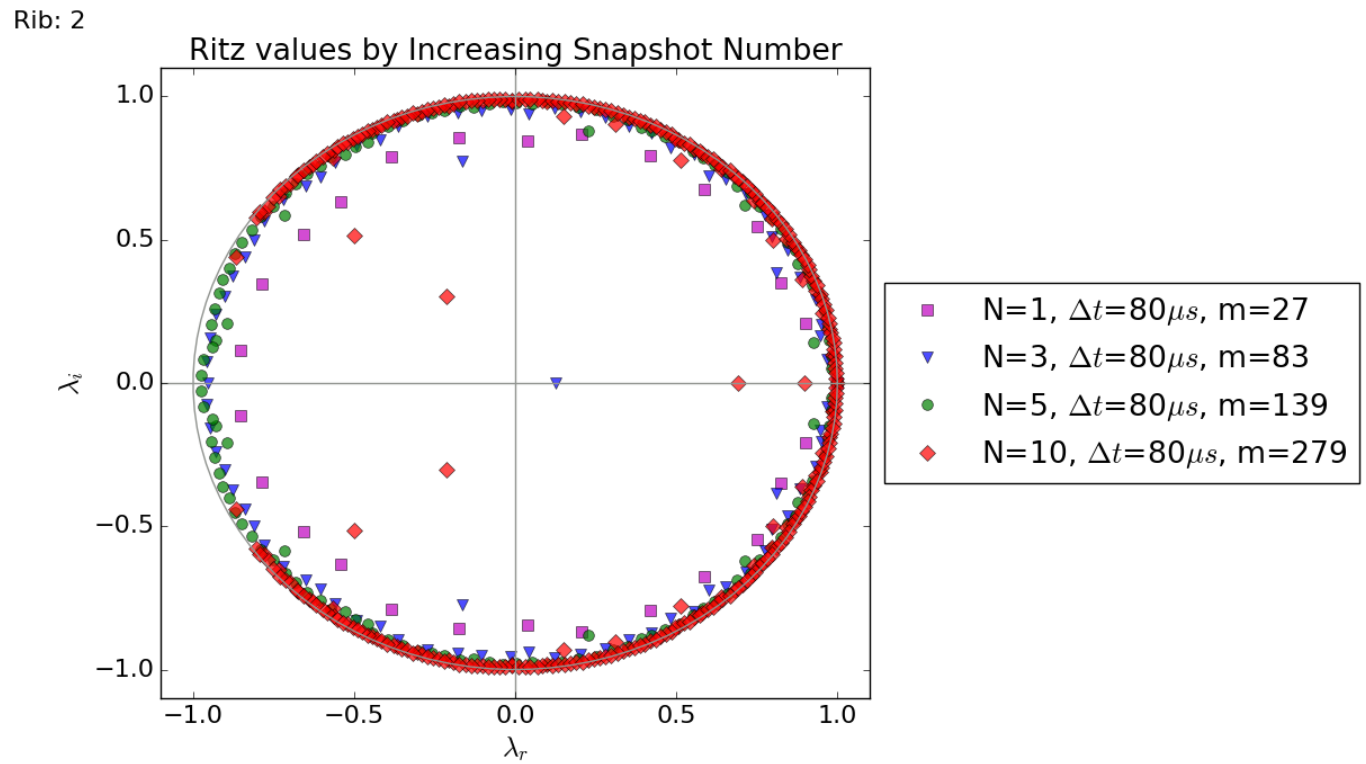

Figure 3.14: Ritz values for $\Delta t=80 \mu s$.

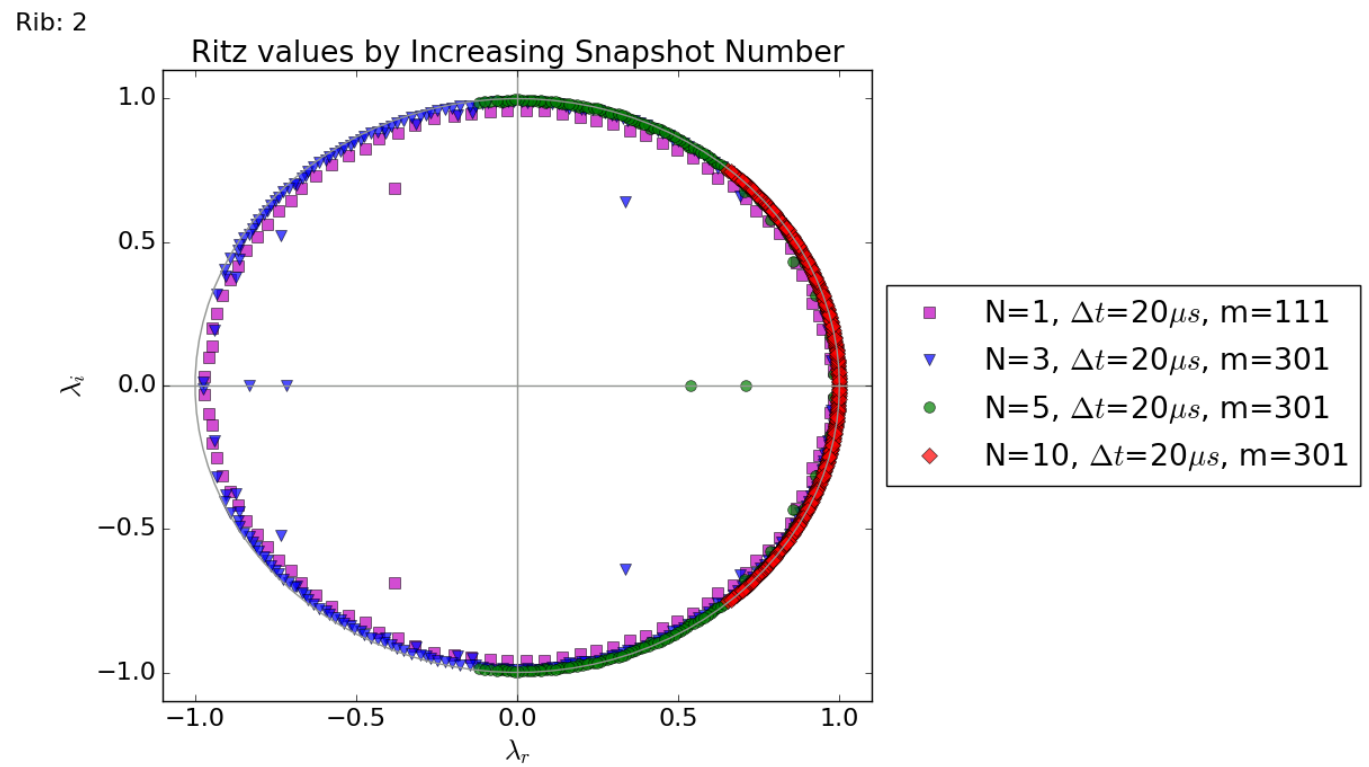

Figure 3.15: Ritz values for $\Delta t=20 \mu s$. 


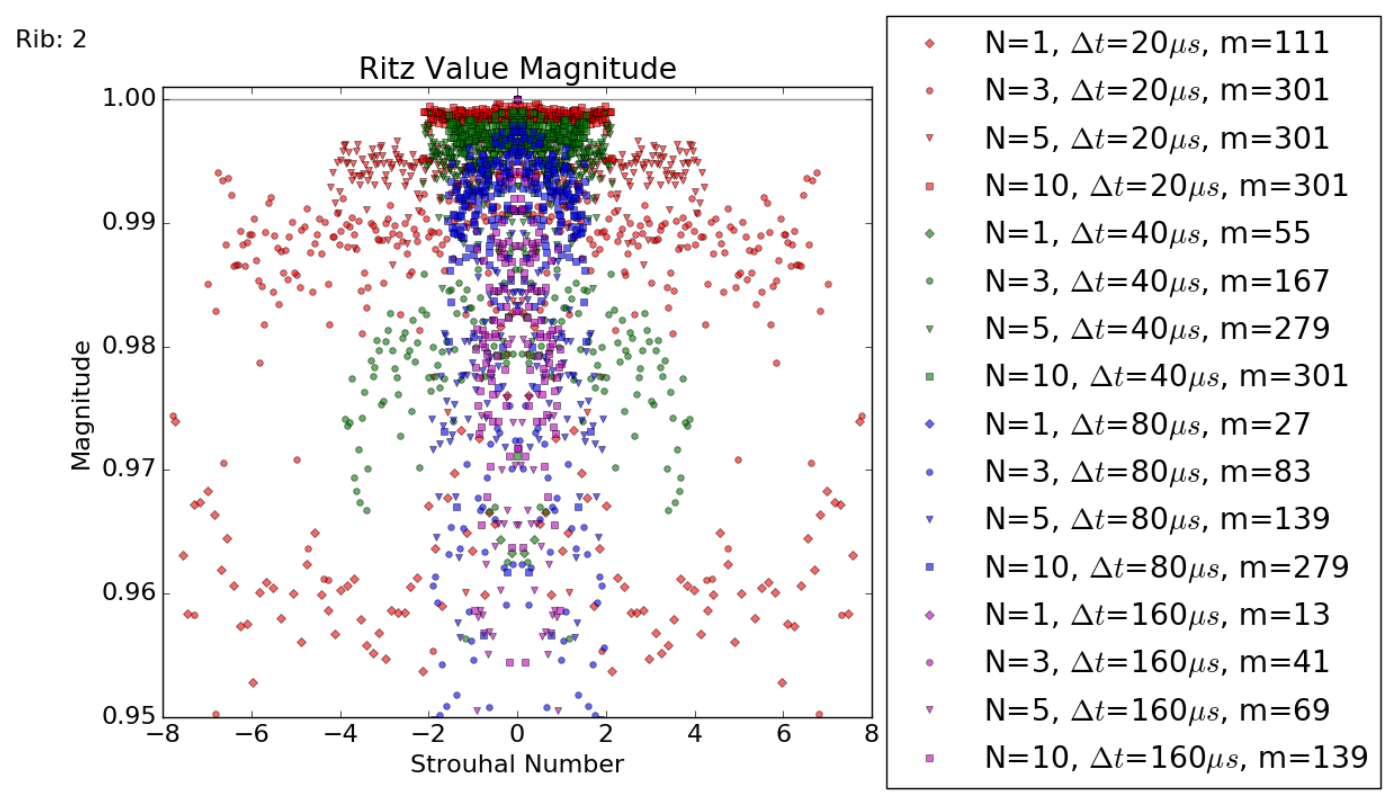

Figure 3.16: Ritz value convergence, all cases. Note the very tightly-constrained y-axis. Colors delineate timestep sizes, shapes delineate number of flow-throughs.

\subsubsection{Modal Amplitude Convergence}

The amplitude of a mode is considered its 'energy', which is exhibited at different frequencies. The computation of these values are covered in section 3.8.3. This metric is the one used to sort the DMD modes into their given names. Mode 0 is the average, and has the largest amplitude in every case, sometimes by several orders of magnitude. As done previously with the Ritz values, exploration of any convergence is performed once by increasing the timestep of the snapshots, and again by increasing the total sampling time, corresponding to columns and rows, respectively, in table 3.5 .

Figure 3.17 shows the amplitude data for the 3 rd column, corresponding to $N=5$ for all timesteps. As the timestep decreases, the spectral bandwidth increases, visible as the data expands in frequency on the plot. For a certain frequency range, from about $\mathrm{St}=0$ to \pm 2 , the $20 \mu s$ and $40 \mu s$ 
timesteps roughly share space on the spectrum, which indicates some level of convergence. This is shared by figure 3.18, which shows the 4th column, corresponding to $N=10$. The absence of peaks in the highest resolution dataset is notable. The data for $N=5, \Delta t=20 \mu \mathrm{s}$ has several peaks around $\mathrm{St}= \pm 3$, which disappear for $N=10, \Delta t=20 \mu \mathrm{s}$. At first glance this would indicate a problem, since their highest resolvable frequency should be identical, but their lowest frequency is not identical. Because of the rank limiting to 301, more low frequency modes are realized on the lower end of the scale, which filter away the higher frequency content, causing the maximum resolved frequency to drop. In both of these images, the lesser resolved points on the table are quite noisy. Both rank limiting and increasing the spectral resolution aid in organizing the higher resolved points.

Figure 3.20 shows the amplitude data for the 2 nd row, $\Delta t=40 \mu s$. As seen previously, the two longer sampled datasets share some of the spectrum, now from $\mathrm{St}=0$ to \pm 1 . In fact, their spectrum is nearly the same in their entirety, with a small separation as the frequency increases. There is a very large difference for datasets of $N \leq 3$, indicating at least 5 flow-through times is appropriate to capture the system, but 10 is superior. Moving to the lowest timestep in figure 3.19, similar results are found, though the very stark difference from $N \leq 3$ seen before is not as severe. This may only be because of the rank limiting imposed on the $N=3$ dataset, which would tend to reduce noise of higher frequencies. Noting the newly-expanded $\mathrm{x}$ axis scale, the spectra still tend to agree around 0 to \pm 1 . Judging convergence of these values is more difficult than the residual or eigenvalue method, and owes some interpretation, even in other literature. The changes in spectral bandwidth from case to case, especially in cases of rank-limiting, make this difficulty worse. In this case the engineer must judge the changes based on a small number of modes close to the 0 frequency. When performing rank-liming as done here, amplitude convergence is perhaps better left to the residual.

The remaining figures representing the other columns and rows can be found in Appendix A. 


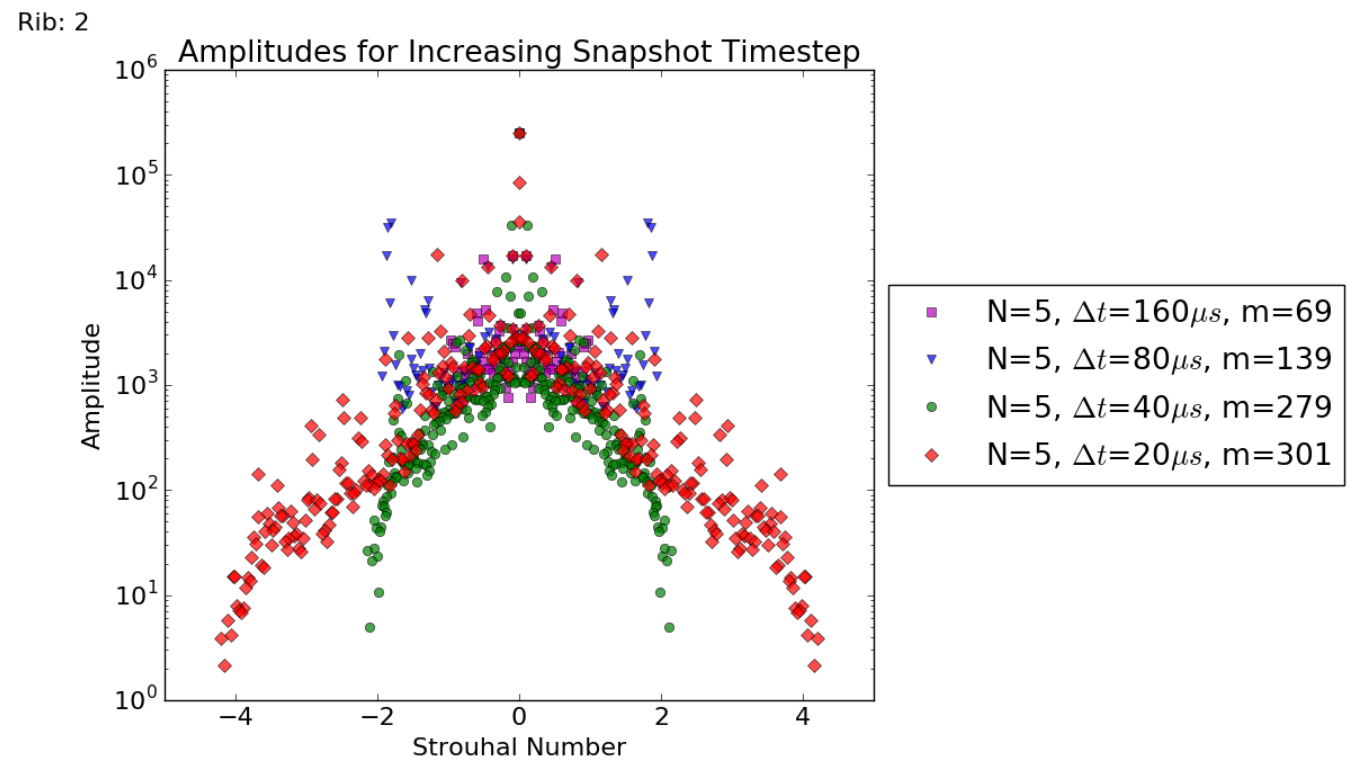

Figure 3.17: DMD amplitude convergence, $N=5$.

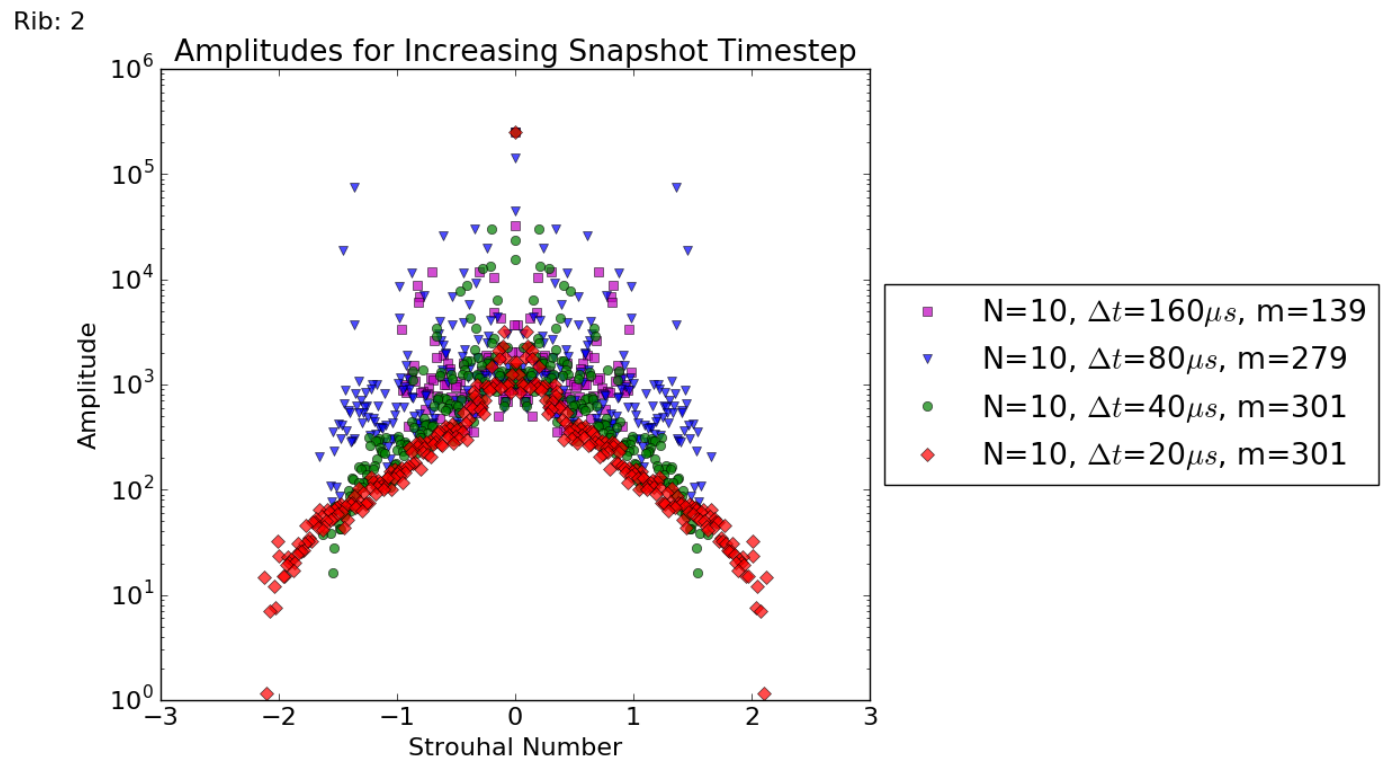

Figure 3.18: DMD amplitude convergence, $N=10$. 


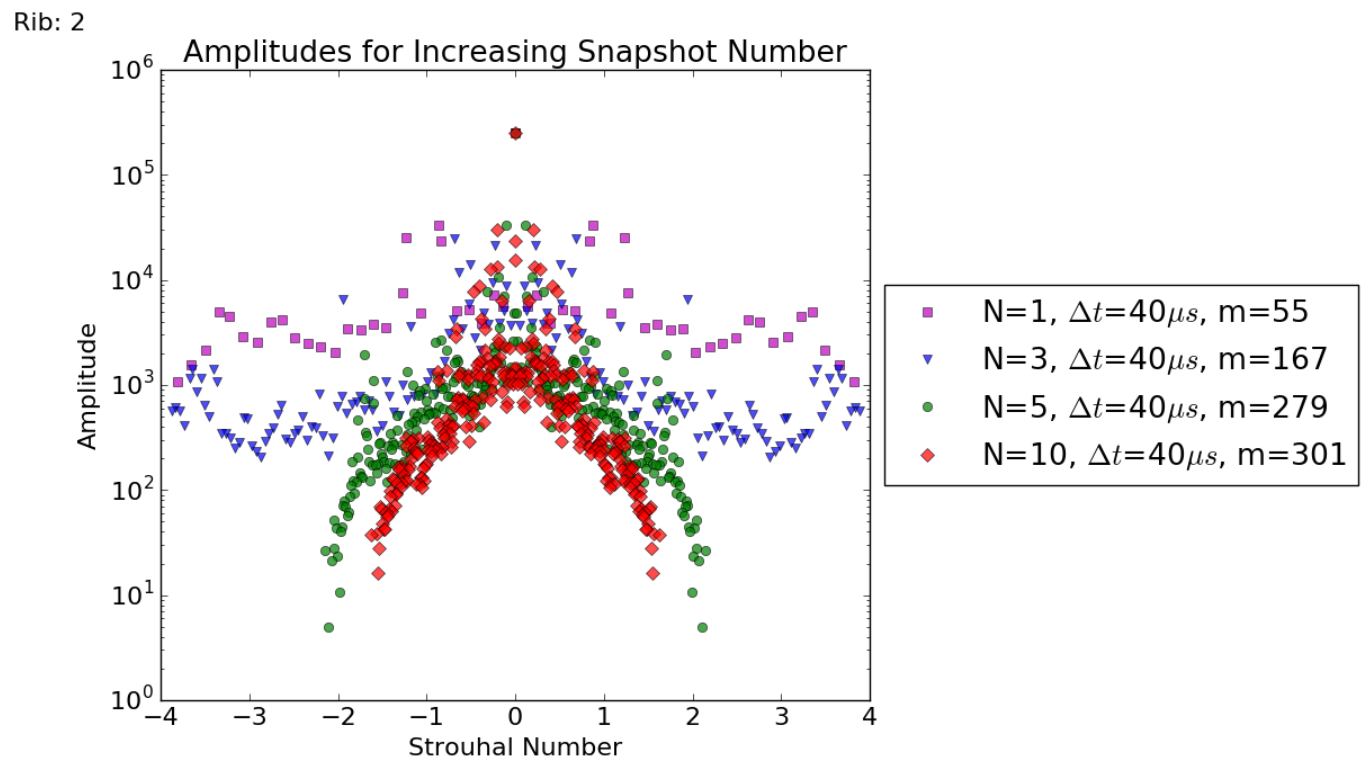

Figure 3.19: DMD amplitude convergence, $\Delta t=40 \mu \mathrm{s}$.

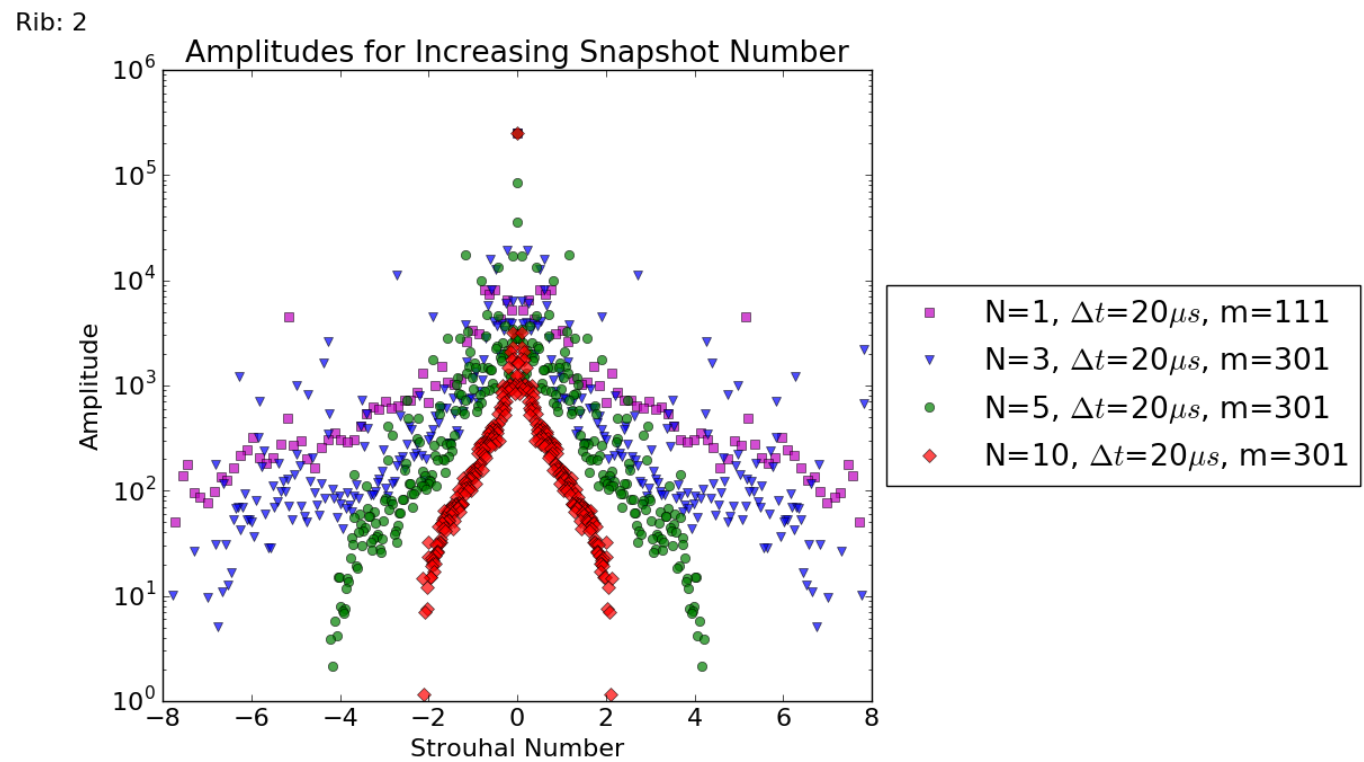

Figure 3.20: DMD amplitude convergence, $\Delta t=20 \mu \mathrm{s}$. 


\subsubsection{Reconstruction Residual Convergence}

The reconstruction residual calculation was introduced in 3.9.3, and is performed here for differing $N$ and $\Delta t$. Figure 3.21 shows the computed residual of all cases in table 3.5. This figure displays several points:

- The minimum attainable residual level depends mostly on $\Delta t$.

- As $N$ increases, the number of modes required for convergence increases.

- Convergence is not always monotonic.

These points mirror the results of [16]. Any timestep can bring about convergence provided enough timesteps are available, but that convergence will be locked to a certain residual level. In other words, for a fixed maximum frequency, additional modes can increase spectral resolution, but the filtering of the original field will always cause loss of information. Increasing the number of snapshots for a given timestep tends to slow convergence. This seems reasonable; additional resolution will allow resolution of more modes. Given this plot one should choose a combination of $\Delta t$ and $N$ which gives convergence and a low residual, indicating good coverage of the spectral bandwidth and a low amount of filtering done by the algorithm. With the knowledge gained from the previous sections, the highest resolution dataset is chosen to use for the remainder of this research, that of $N=10$ and $\Delta t=20 \mu s$. 


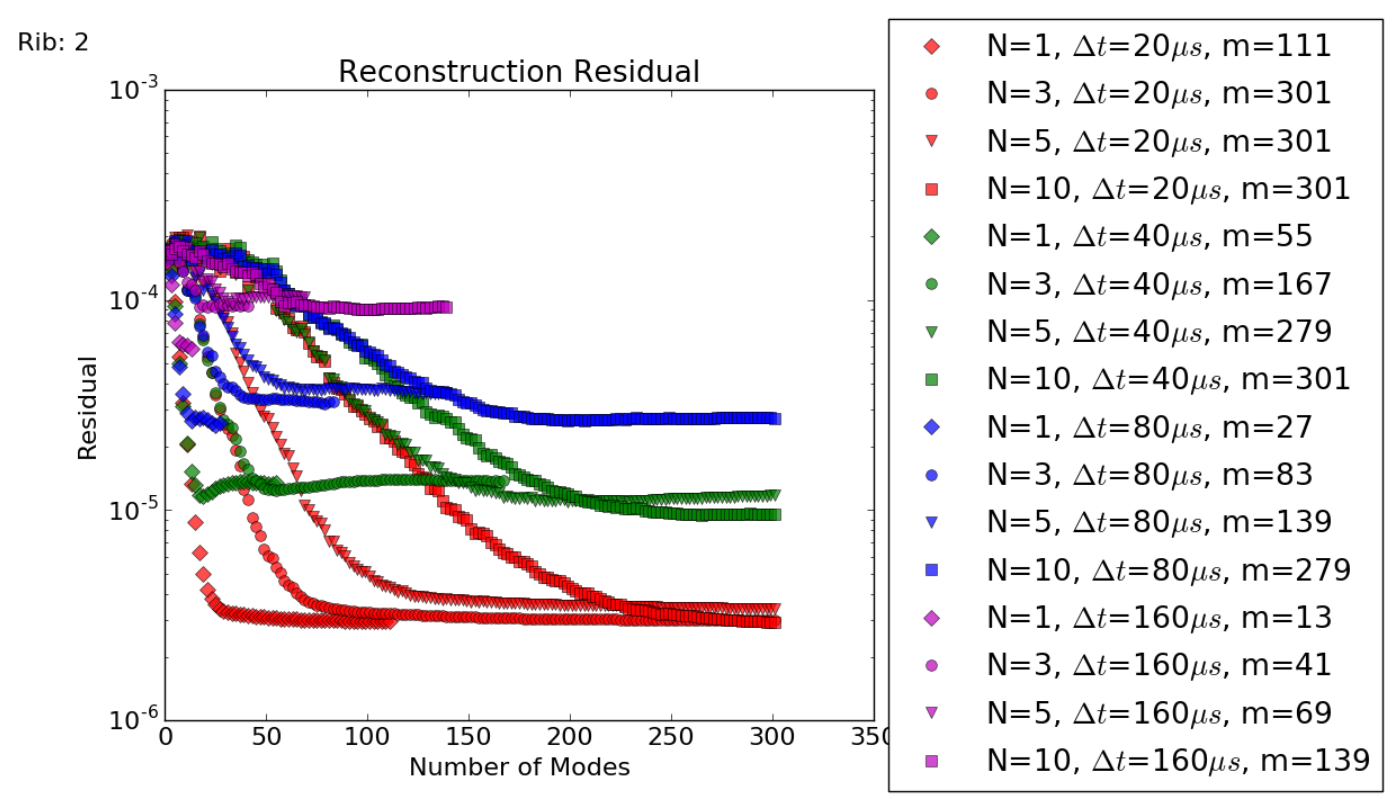

Figure 3.21: Reconstruction residual of the final timestep in the snapshot series. Colors delineate timestep sizes, shapes delineate number of flow-throughs.

\subsubsection{Reconstruction Visualization}

The quantitative metrics given in previous sections are excellent at showing the ability of different parameters to recreate the solution from measurements. This section gives a more qualitative feel to the reader as to what differing reconstructions look like. The first question one may have is are there enough probes in the grid to resolve the scales in a manner similar to the original mesh. Figure 3.22 shows a comparison of a time snapshot between the original mesh (A) and a nearly identical time point using the DMD grid (B). Note that (B) is merely plotting the measured solution on the probe grid; no DMD has been performed. The isosurface plotted is of temperature at 315 K. The surfaces are extremely similar by inspection. This helps make the low residuals computed earlier possible.

Also of interest is the flow reconstruction performed with different numbers of modes. As an ex- 
ample, Figure 3.23 shows the reconstruction with 3 different numbers of modes $-301,27$, and 7 - compared to the primitive measured values. The great similarity between the 301 mode reconstruction and the primitive is notable.

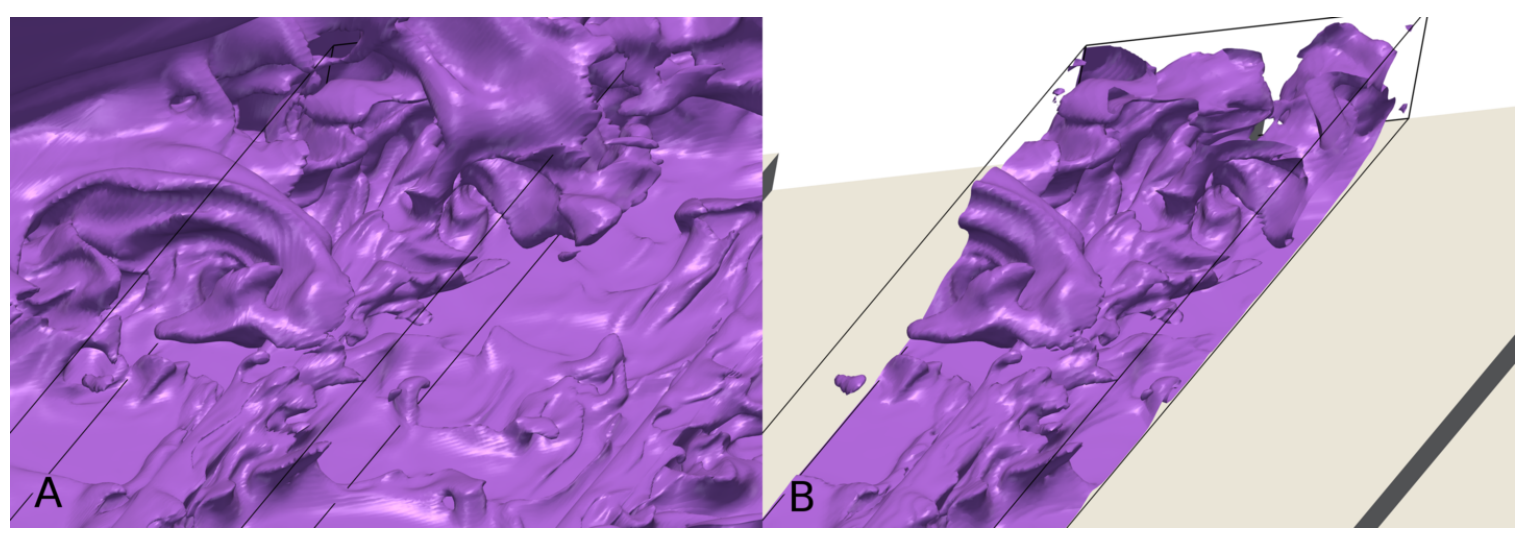

Figure 3.22: Isosurface temperature ( $315 \mathrm{~K})$ shown on the CFD grid (A) and a nearly identical timestep on the DMD probe grid (B). Flow moves from left to right, so we are viewing the swept end of the rib. The thick black line outlines the DMD volume. 


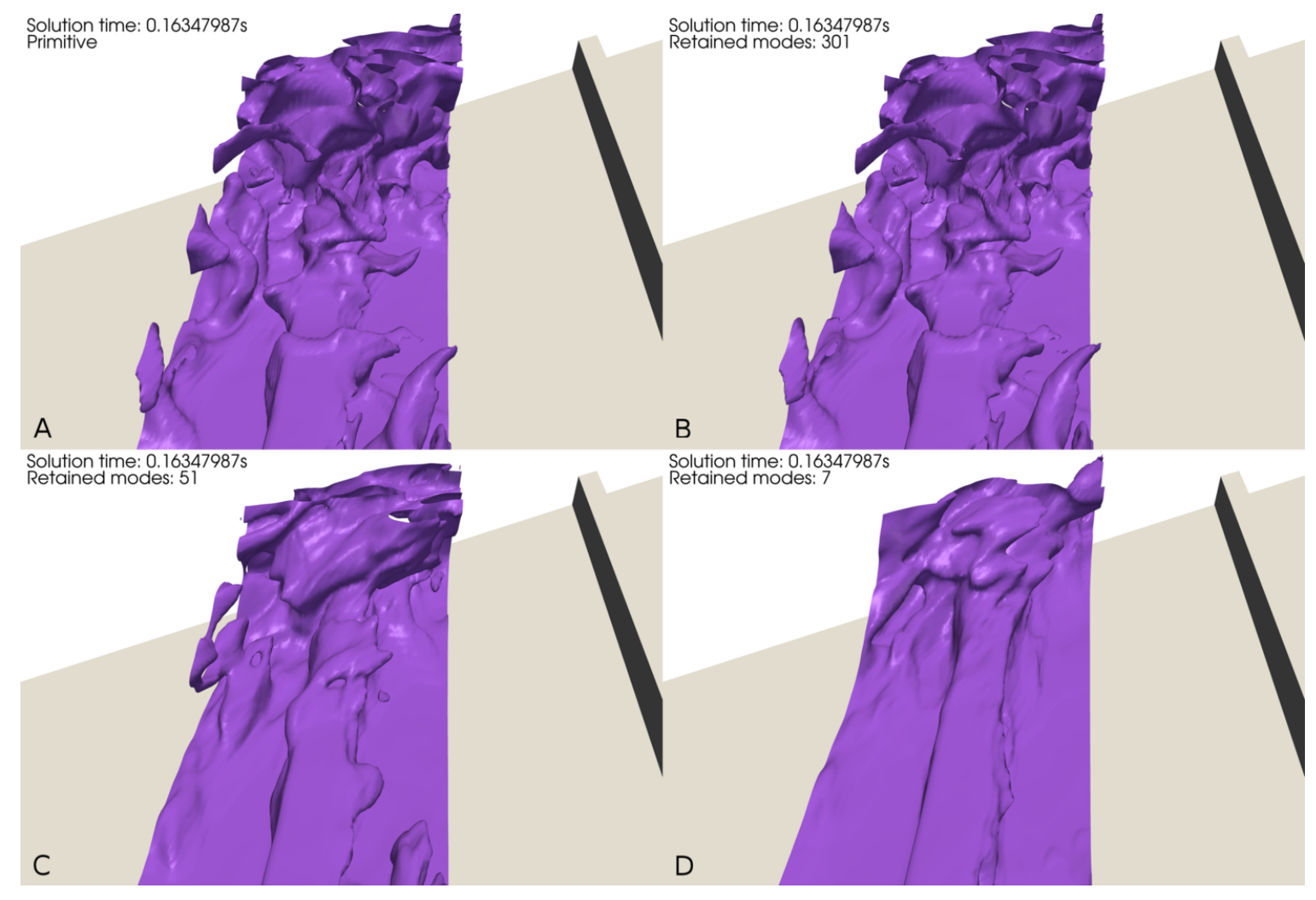

Figure 3.23: Temperature isosurface of $315 \mathrm{~K}$ for differing numbers of retained ranks, as well as the primitive field. Flow moves from left to right, so we are viewing the swept end of the rib. The top two panels are nearly equal, as the number of ranks is high in $\mathrm{B}$. The bottom two panels $\mathrm{C}$ and $\mathrm{D}$ give perspective on the different kinds of content the modes can produce. 


\section{CHAPTER 4: RESULTS}

An industrial analysis serves as a showcase for the DMD methodology proposed. The processes' steps, as stated earlier, are:

- Compute heat transfer on a baseline RANS analysis

- Execute an LES computation, extracting snapshot data for the DMD

- Perform the DMD analysis and assess which modes may be important to heat transfer

- Morph the duct geometry using the DMD mode shapes in an effort to excite those modes

- Compute heat transfer on modified geometries using RANS and assess any differences.

An obvious criticism of this procedure would be the usage of steady-state, non-scale-resolved simulations to validate modifications to modal structures identified with a transient technique. A true validation would involve a follow-up LES simulation with the morphed geometry, which could identify exactly if a modal structure shows an increased participation in heat transfer. However, due to limited available computational resources, a second LES simulation is currently infeasible. Therefore strong caution must be used in assessing the impact of the geometric changes, and more research will be required to show if any effects are a direct cause of changes in desired modes. The majority of these results will be reported for the area around the second rib. While data was taken to construct DMD analyses for all ribs, the morphed comparison cases were only performed on the second rib. This should represent a rib in the mostly likely industrial scenario, as it has a rib upstream and downstream of it. 


\subsection{RANS Baseline Case Results}

\subsubsection{Convergence}

This case exhibited some 'transient' phenomena on the ribs, as some heat transfer values show oscillations. However since their amplitude is quite low and they do not grow or decay, the run is deemed converged. It is not uncommon that CFD simulations exhibit this behavior; it is merely an artifact of performing a steady simulation on a transient nonlinear system. Figure 4.1 shows the heat transfer iteration history, while 4.2 shows the residual history. The rib surfaces are split into front, top, and rear panels.

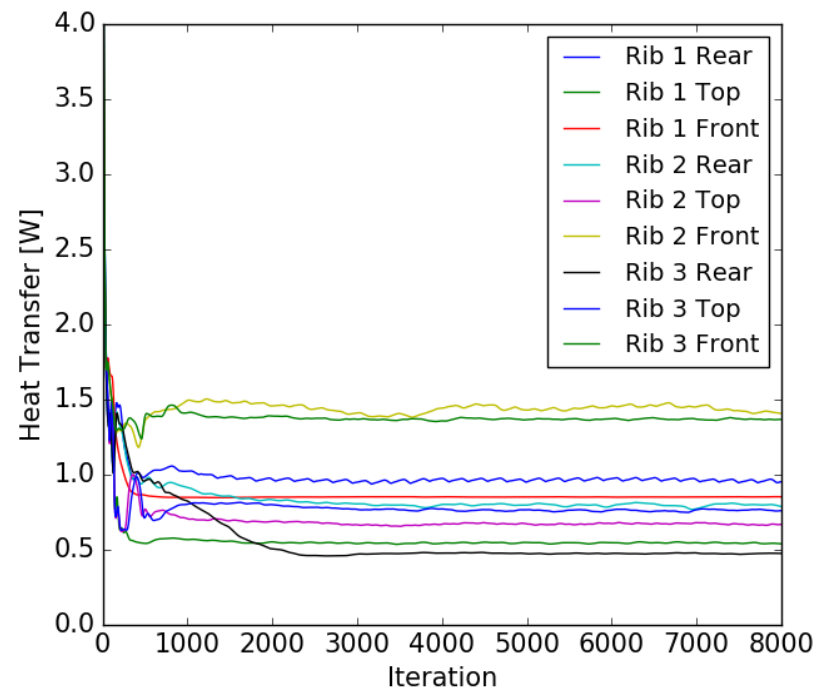

Figure 4.1: Heat transfer convergence, RANS baseline case 


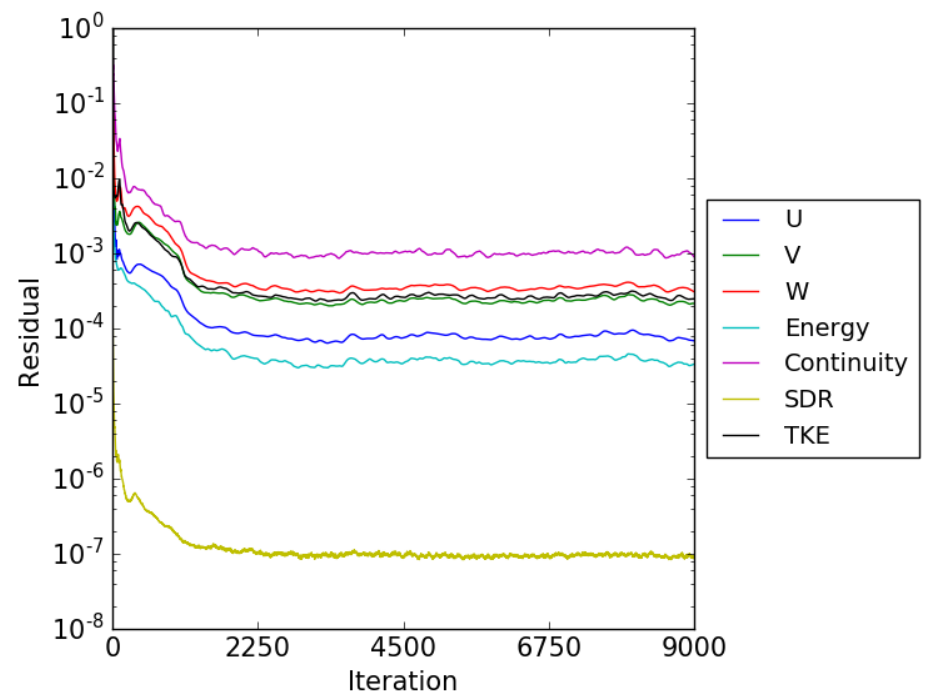

Figure 4.2: Residual convergence, RANS baseline case

\subsubsection{Pressure Drop}

Figure 4.8 shows the section-averaged pressure in the domain along the axis (along with the LES case, discussed later). The dashed lines indicate the leading edges of the ribs. The vertical line indicates the position of the porous damping zone. The (Darcy) friction factor computed from this analysis, 0.0686 , is lower than reported in [2], however this is reasonable since that case involved 2 walls with ribs, compared to the one here.

\subsubsection{Flowfield Features and Description}

Figure 4.3 shows the structures in the flow surrounding rib 2. The front of the rib produces a small vortex near the bottom of the channel (red), which spills into two streams along the span. One leaves the rib about midway, and the other leaves at the swept (trailing) end of the rib. The first 
stream joins the large vortex resulting from the leading edge of the first rib, and the second joins the large vortex resulting from the center of the first rib. The streamlines from the top of the rib (gold) tend to mix with the streamlines from the rear of the rib (blue), though the rear streamlines tend to form the core of their shared vortices. The right side of figure 4.3 shows these streamlines combined with an isosurface of Q-criterion, which gives an idea of flow structures that many not be visible with the streamlines. At the bottom of the image, the main trailing vortex starts from the rib corner. Just inboard is a smaller vortex which counter-rotates. This structure will be important in the morphed cases, as it is close to the surfaces being manipulated. As can be seen in the upperright of the image, many of these vortices twist together to eventually become the primary vortex of the channel, should it be longer as many IDC ducts are. These vortices form the primary flow features inside the DMD domain, and have very strong effects on the heat transfer of the channel base, as seen later.

Figure 4.4 shows the streamwise vorticity component, which gives a good idea of the directionality of the vortical structures. The counter-rotating vortices described previously are plainly visible near the bottom. Also visible is the vortex contained behind the rear of the rib emerging from underneath the leading edge vortices near the mid-span. 


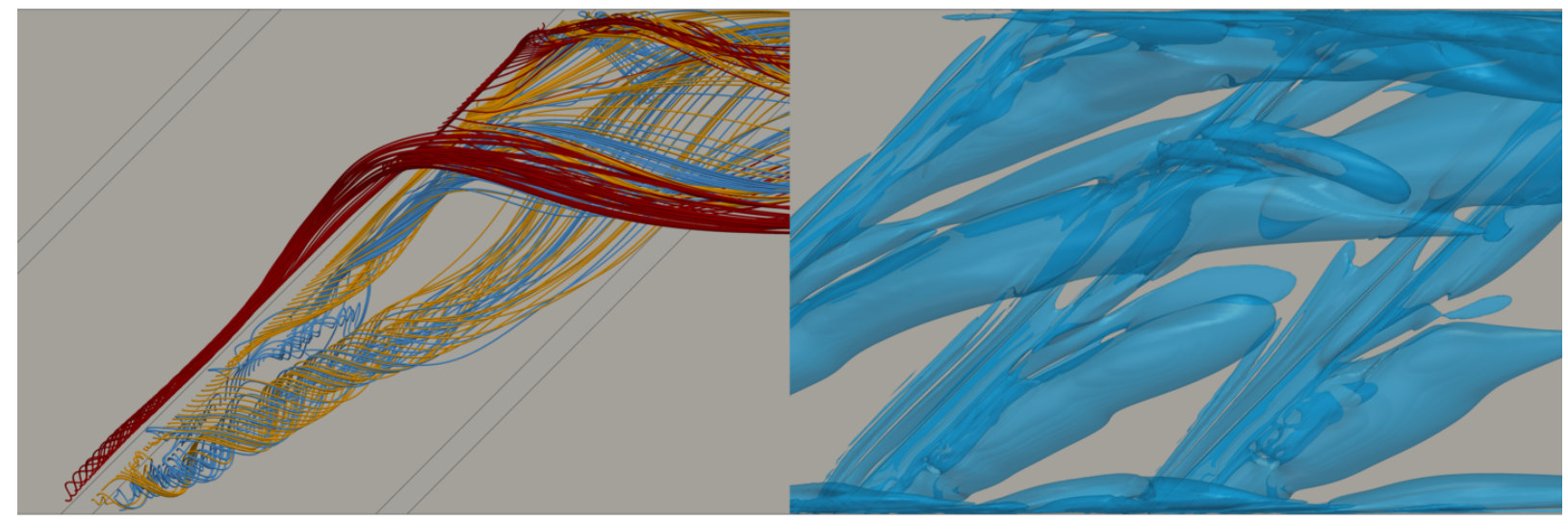

Figure 4.3: Flow structure, RANS baseline case. Flow moves from left to right. Red streamlines are seeded from the front of the rib, gold from the top, and blue from the rear. The blue isosurface (right) is of Q-criterion (rotational). The leading edge vortex stream is visible in red. The trailing edge has two vortices; the larger near the bottom and a smaller inner which moves behind the rib.

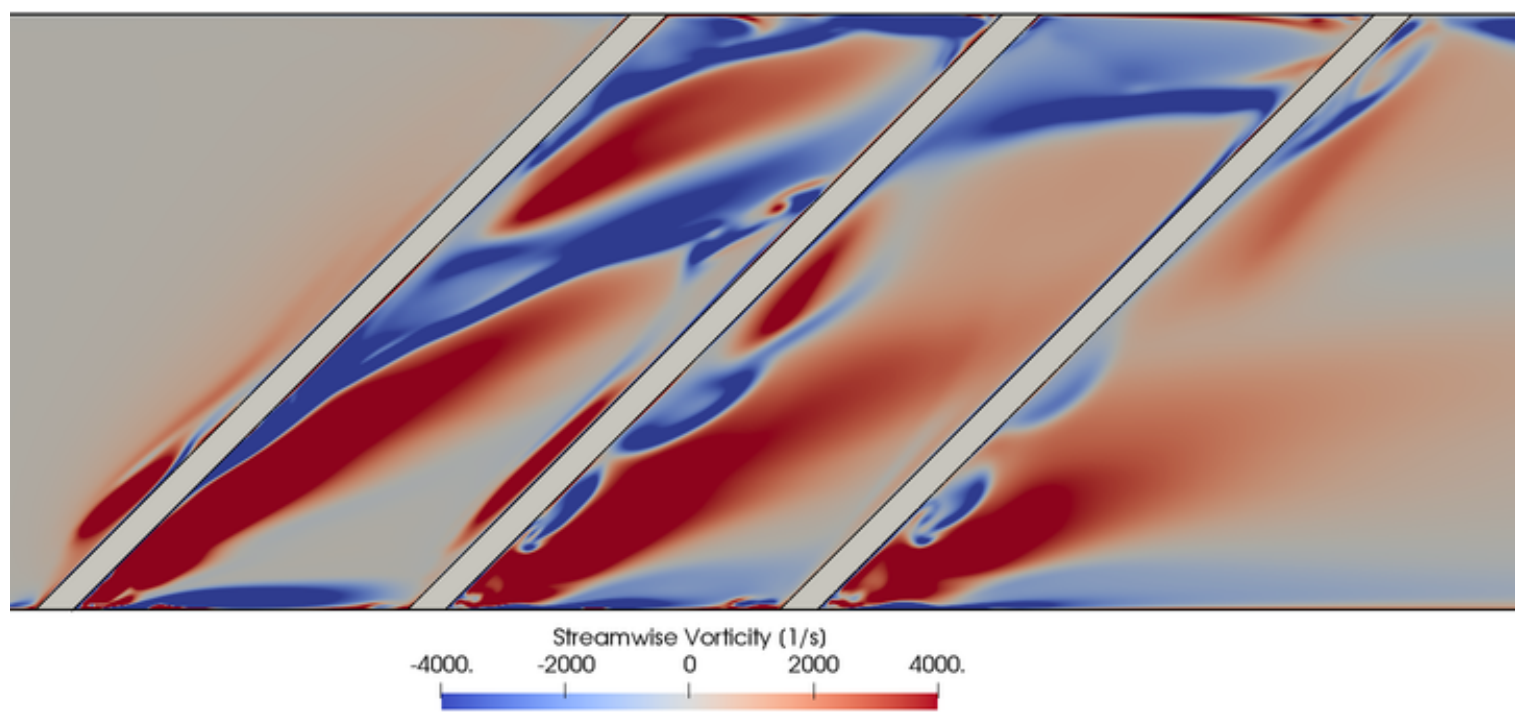

Figure 4.4: Streamwise vorticity component shown on a plane section half way up the rib height. Flow moves from left to right, with positive vorticity. 


\subsubsection{Heat Transfer}

Figure 4.5 shows a top-down view of the Nusselt number ratio on the turbulated surface, and figure 4.6 from the front and rear. The ratios here are similar to those found in [33] and [52], though some difference occurs due to the developing nature of this flow. Each pitch has structural commonalities towards the right wall (bottom of the image), which fade to uniqueness towards the left wall (top of the image). This is due to the development of the large vortex which would eventually dominate a longer channel's flow character. Visible are the primary (red-white) and secondary (blue-white) trailing vortices identified earlier. There is some impingement on the second rib center due to flow moving over the vortex trailing the first rib. A comparison to the LES case and notes on literature comparisons are given in section 4.2.3.

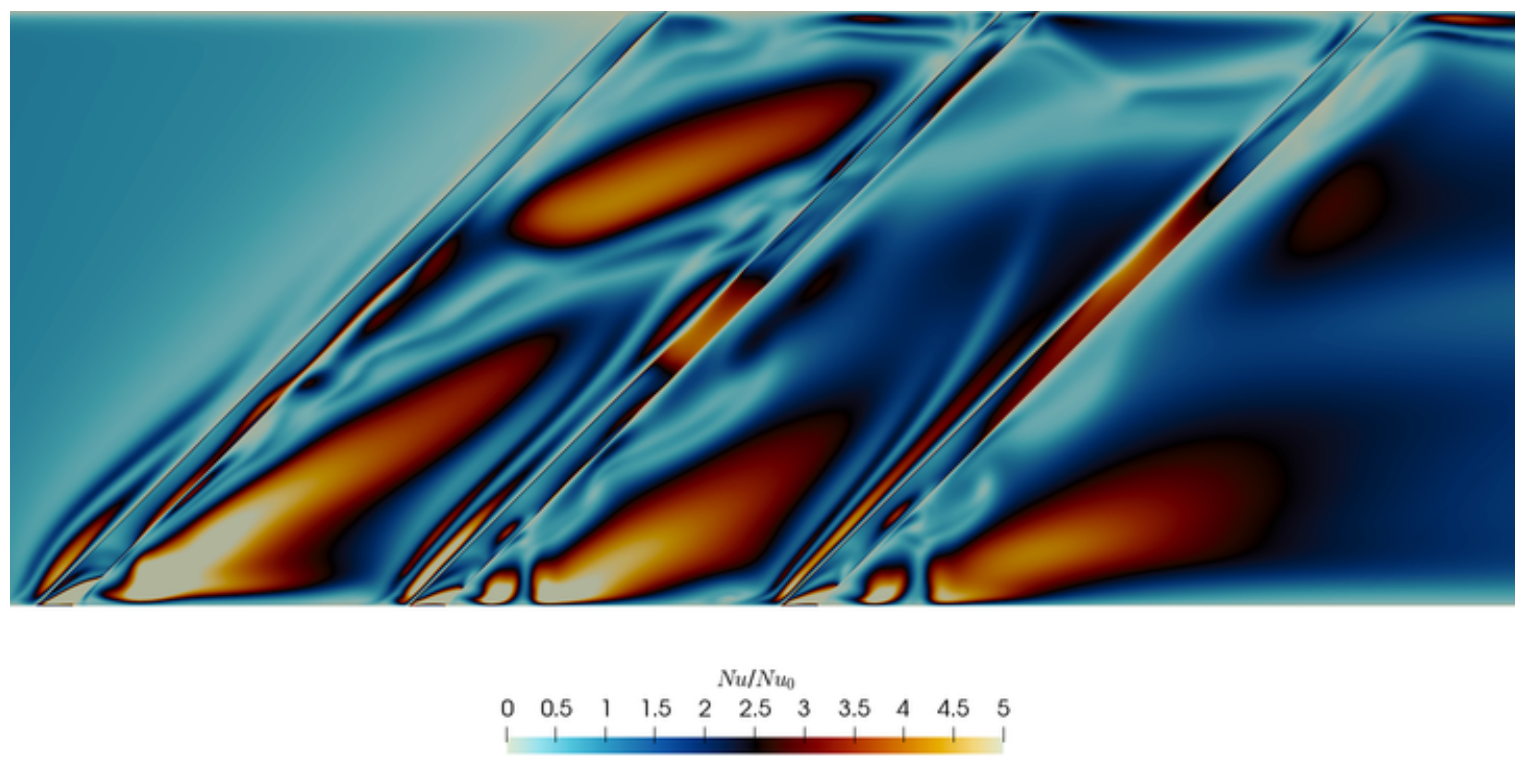

Figure 4.5: Nusselt ratio, RANS baseline case. Flow moves from left to right. 


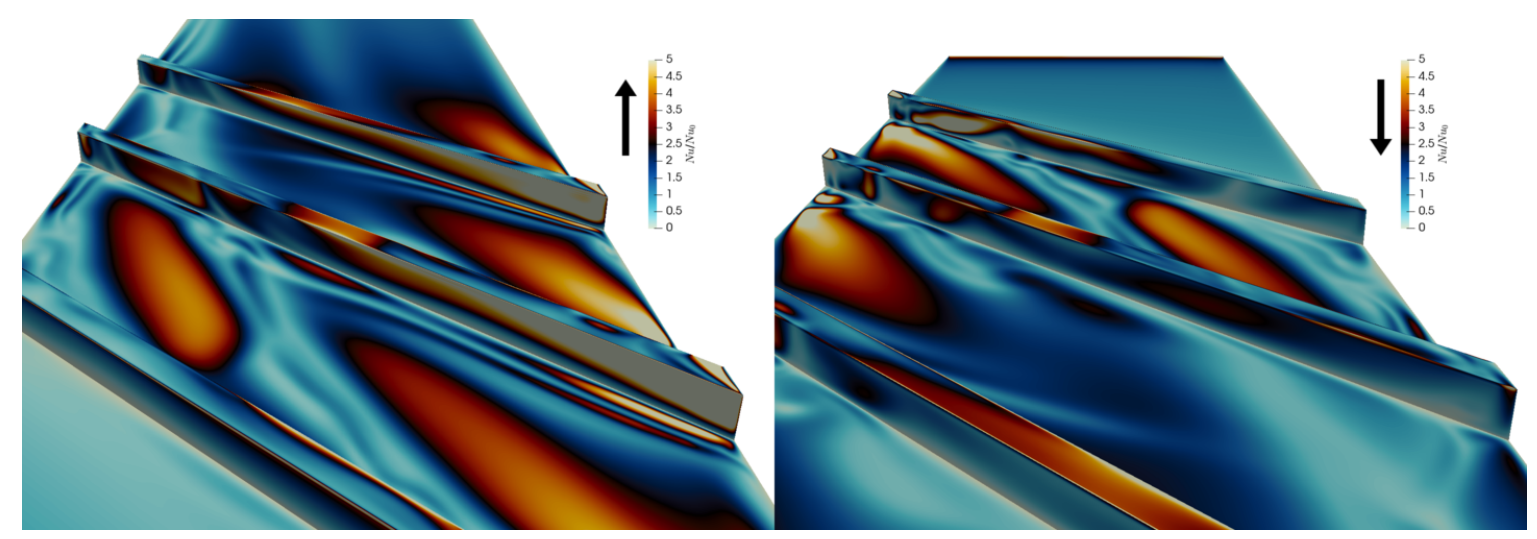

Figure 4.6: Nusselt ratio, RANS baseline case. As viewed from the front (left) and rear (right). Flow moves with the arrow.

\subsection{LES Case Results}

\subsubsection{Statistical Stationarity}

The statistical stationarity of the time signal of three-dimensional velocity at the grid points visualized in figure 3.4 are evaluated using the method in section 3.6.3. Unfortunately due to time and computing restraints, only about $20 \%$ of the probes reveal stationary signals at the time of writing. The LES case was run to about 5 flow-through times, so significant development has occurred, but due to the criterion provided by the Reverse Arrangements test, statistical stationarity cannot be claimed. The time signal of two probes behind the third rib can be seen in figure 4.7. While the running time average shows some leveling, it clearly requires additional time to become quite flat. The variances of these signals also show the need for additional computing time. However since the fields clearly show signs of some level of loose convergence. The lack of convergence will make the evaluation of the DMD modes more suspect, but since the system is somewhat converged, the regression may be able to handle the smaller transient changes still occurring. 

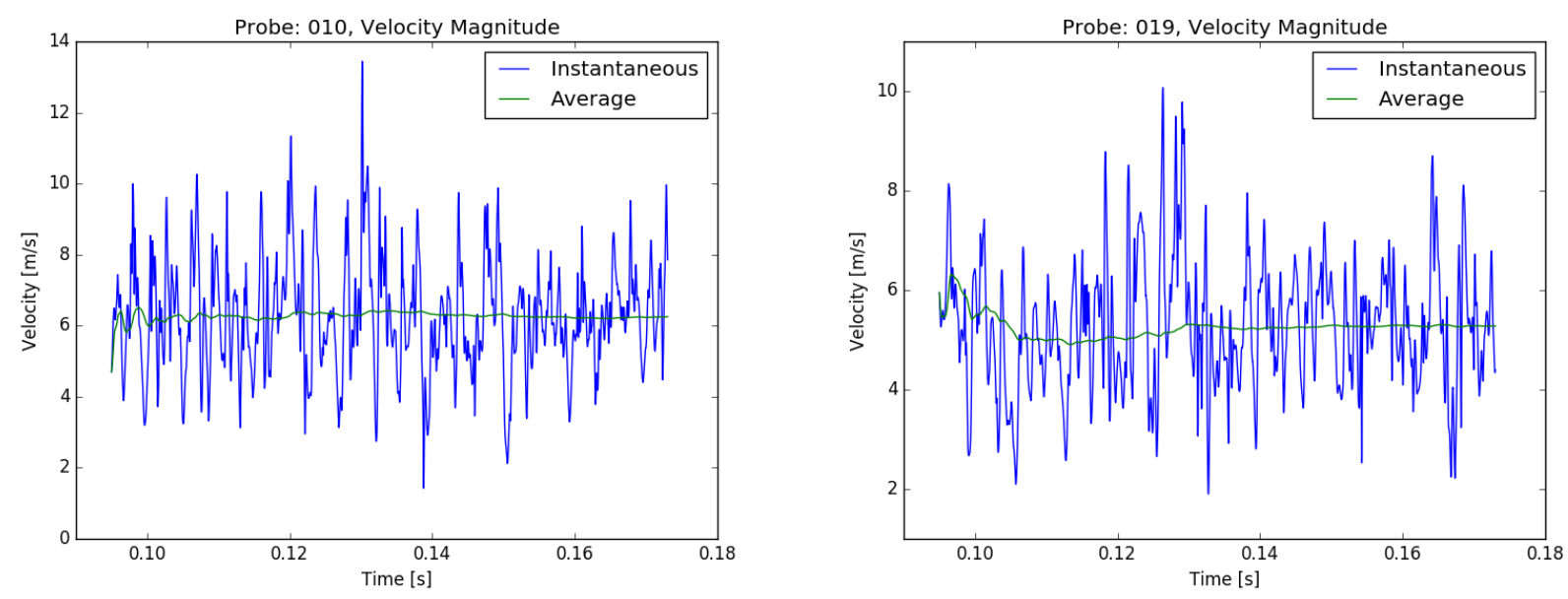

Figure 4.7: Velocity time history and running average of two probes behind the third rib. The average is somewhat flat nearing the end, but still requires additional time to be stationary.

\subsubsection{Pressure Drop}

The friction factor in the LES case was 0.0611, slightly lower than the RANS counterpart. Seen in figure 4.8, much of the difference appears over the first rib. Unsurprisingly, this is where the two simulations differ in heat transfer, and by extension flow structure, the most. How far the first rib's vortex moves toward the surface between ribs 1 and 2 is the primary difference at the point where the curves diverge. The RANS case shows the stream moving very close to the surface, increasing heat transfer significantly, whereas this effect is far lower in the LES case. In addition are the differences mentioned earlier on the leading edge vortex. Downstream of this point, the two pressure curves are mostly identical. Friction factors can be viewed in table 4.4. The difference in friction factor may indicate enough of a difference to throw off the morphed results. If the initial RANS simulation does not perform close enough to the LES case, the modes extracted may be too different to the RANS case to have the desired effects. 


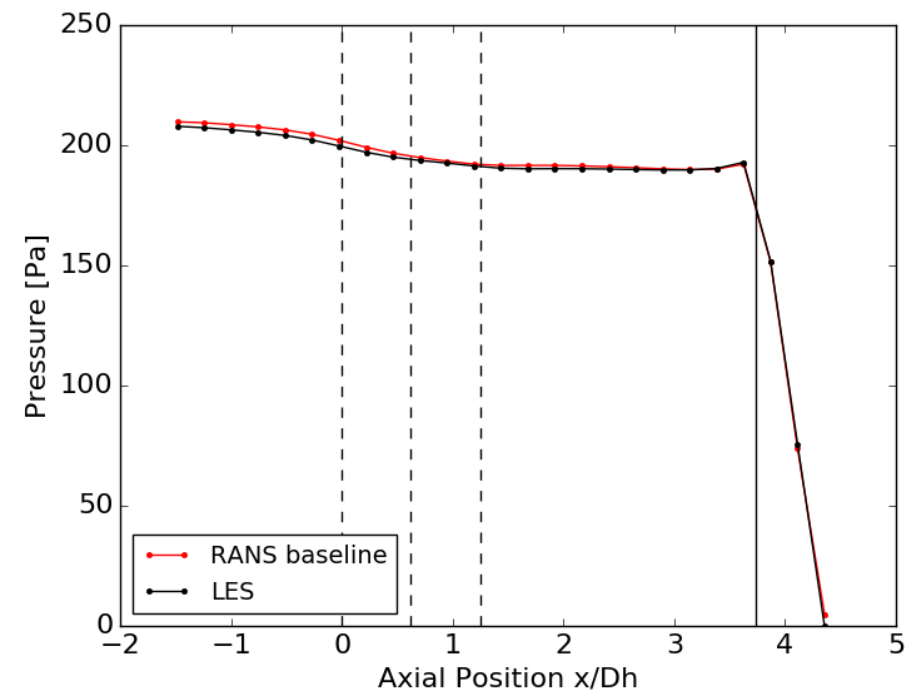

Figure 4.8: Pressure drop, RANS baseline and LES case. The dashed lines represent the rib leading edges. The solid vertical line represents the beginning of the porous damping zone.

\subsubsection{Flowfield Features and Description}

Many of the flow features present from the RANS case are reproduced in the LES case, but with differing positions and strengths. Most of the large vortices produced near the swept side of the ribs are smaller than their RANS counterparts, contributes to the increased heat transfer along the very bottom of the channel in the LES case. The largest difference is the point at which the leading edge vortex climbs over the rib. The LES case predicts this to happen much later, almost to the end of the rib. The RANS case predicts the vortex trailing the first rib to be slightly larger near the second rib, causing flow moving over the vortex to dive further downstream. As a result, the leading edge vortex peels upward into a stream earlier than in the LES case. There, the first rib vortex is slightly shallower, allowing flow above it to move more smoothly toward the second rib and spreading out rather easily. These streamlines carry far more momentum and block the path of the leading edge vortex, causing it to join the rest of the flow near the wall instead. This comparison is best viewed 
in figure 4.10. The LES streamlines more closely resemble the ones of Chanteloup et al. [10], but comparison should be done cautiously due to a Reynolds number difference, slightly different rib geometry, and fewer ribs in the present model. The behavior of the LES flow over the rib, depicted in the gold streamlines, is as described in [10], lining the recirculation vortex behind the rib until it hits the side wall or joins the remaining flow, all while growing in size.

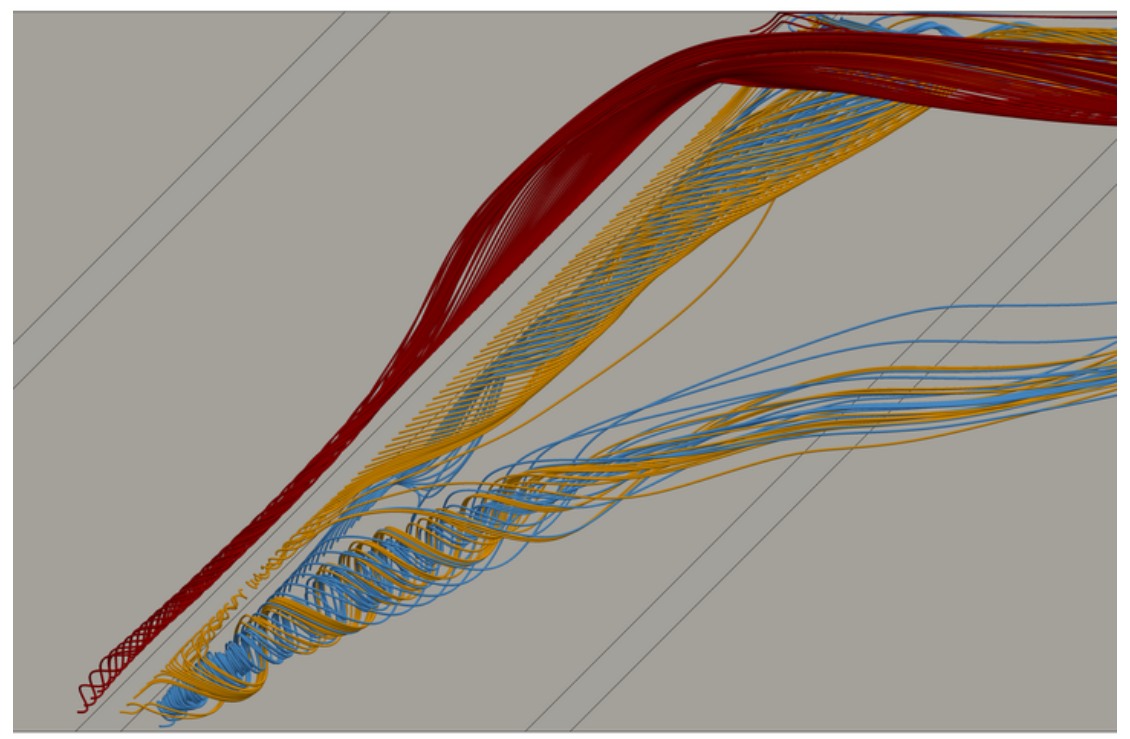

Figure 4.9: Flow structure, LES case. Flow moves from left to right. Red streamlines are seeded from the front of the rib, gold from the top, and blue from the rear. Note the delay in the downstream movement of the red streamlines compared to RANS. 


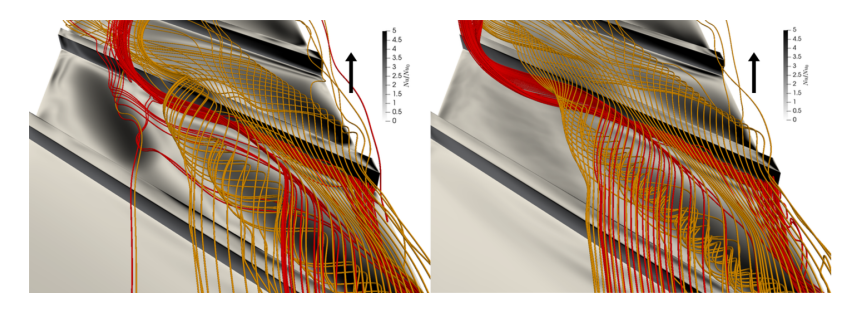

Figure 4.10: RANS (left) and LES (right) rib 2 impingement comparison. These images show how the streamlines that move over the rib 1 vortex (gold) and hit rib 2 in a different way, causing the leading edge vortex (red) to peel over rib two in different positions. Flow moves with the arrow.

\subsubsection{Heat Transfer}

Figure 4.11 shows a top-down view of the Nusselt number ratio on the turbulated surface, and figure 4.12 from the front and rear. The largest change from the baseline is the significant drop in heat transfer aft of the first rib at the center of the channel. LES predicts the vortex in that location to stay further off of the surface, resulting in less impinging flow. One pitch later, the same location shows a small increase compared to the RANS case. Most literature does not show this second area of heat transfer, however most of the reviewed literature also do not show developing flows. Yet another pitch later, the area seems to fade somewhat. The large vortex structures moving over the surface at the top of the image start the channel secondary flow pattern. It is safe to say the impinging effect aft, center-channel of rib 2 would not be present much further down the channel, had the ribs continued. The distribution of the heat transfer appear very reasonable compared to [10], though the values are somewhat different due to developing flow and Reynolds number differences. A surface average Nusselt number was compared between the LES and RANS cases, finding the LES to predict a lower value by 5.7\%. This is a better result than the difference in friction, however due to the difference in the heat transfer fields as shown, some of this low difference may just be because of offsetting errors. 


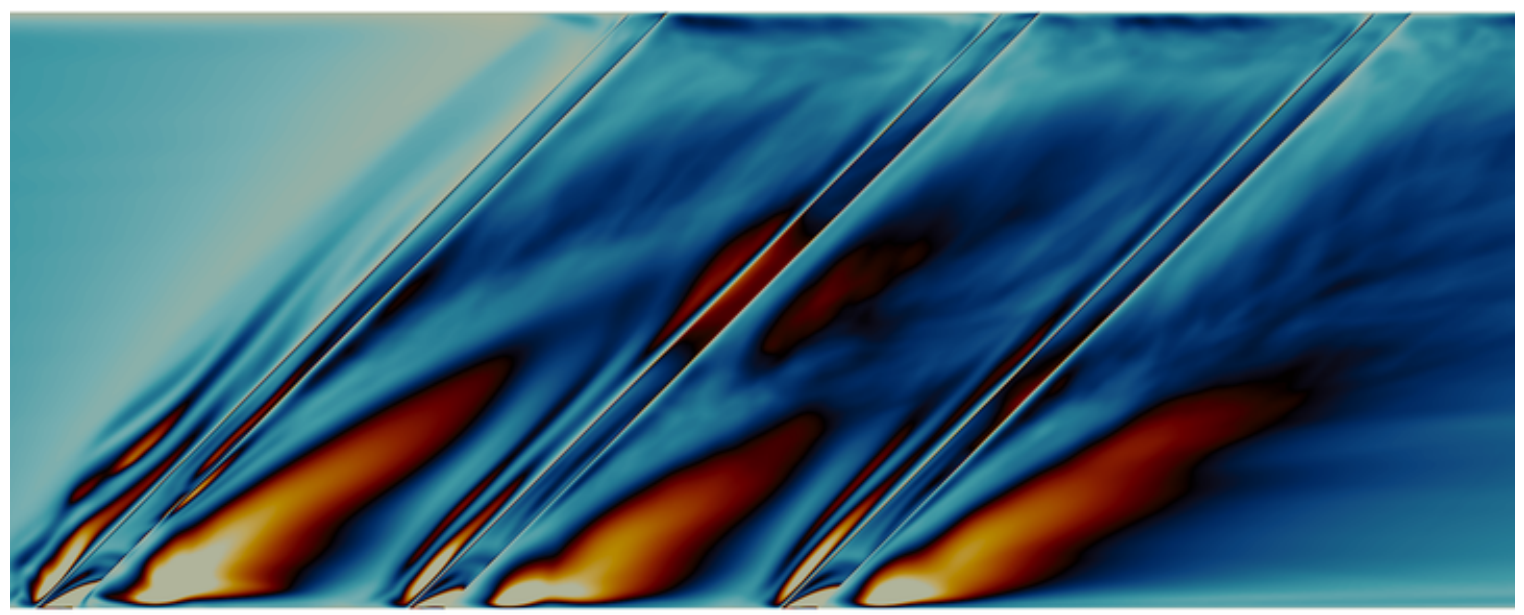

$\begin{array}{lllllllllll}0 & 0.5 & 1 & 1.5 & 2 & 2.5 & 3 & 3.5 & 4 & 4.5 & 5\end{array}$

Figure 4.11: Average Nusselt ratio, LES case. Flow moves from left to right.

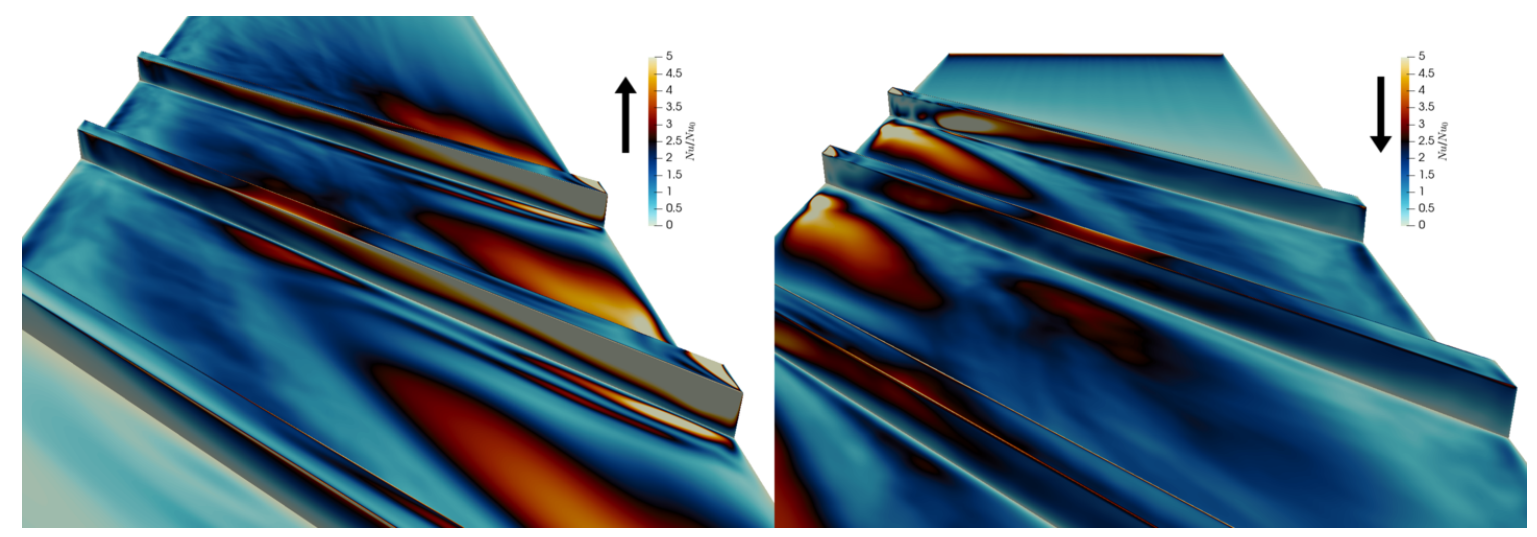

Figure 4.12: Nusselt ratio, LES case. As viewed from the front (left) and rear (right). Flow moves with the arrow. 


\subsection{DMD Results}

The DMD algorithm is executed on the results from the LES case utilizing the methodology from section 3.8.4. This results in a prediction of heat transfer for each mode included in the decomposition. The value of heat transfer predicted by each mode is normalized by the value of heat transfer predicted by all modes for simplicity. They can also be normalized by the heat transfer predicted from the primal flow field, however in practice the two values should be nearly identical, so this choice becomes arbitrary.

Figure 4.13 shows the normalized heat transfer for all modes. As shown, the first mode (the mean) supports the vast majority of heat transfer. This makes sense, since the target quantity is, after all, an average of heat transfer. The remaining modes carry only a few percent of the total heat to give nearly perfect accuracy. In fact, modes after 100 or so contribute nearly nothing to the total heat transfer, as the previous modes are sufficient to almost perfectly predict the primal. This agrees with the results of the DMD convergence tests, which also indicated a diminishing returns on residual after about 100 modes.

Figure 4.14 shows a 'zoomed' view of the plot containing all of the modes. Here we can see that there are occasionally stark changes in the heat transfer prediction. Recall that the mode numbering is chosen such that 0 is the mean, and the remaining modes come in complex conjugate pairs. So a 'new' mode shape is defined with an odd number. The most obvious feature is the lower spike at mode 13. Since the heat transfer prediction with that mode appears to drop, one might conclude that mode acts to reduce the heat transfer of the flow field. The peak heat transfer sits barely above one at mode 33, which one might conclude enhances heat transfer. In order to test the theory of using mode shapes to affect heat transfer in a predictable way, both modes 13 and 33 will be used to morph the mesh. An additional way to view the mode's contribution to heat transfer is presented shortly, which has more merit.

In addition, it would be prudent to test a mode shape which shows no interesting character in the 
above analysis to see any effects on the heat transfer. The mode selected was 99.

Another way to view the heat transfer from the different modes is from the superposition perspective. That is, each additional mode adds or removes something from the linear system which may be desirable or not. By subtracting the prediction of a mode from the one that came before it, we could assess the impact of the addition of the new mode onto the system. This may aid in determining modes which increase heat transfer a large amount, ones that decrease the heat transfer, and ones that provide nearly no benefit. Figure 4.15 shows just this. Since the modes will appear in complex pairs, it's not physical to separate them, so the subtraction is performed between odd valued modes. The only exception is the first subtraction, which is the first mode minus the mean (zeroth) mode. The mesh can only be deformed based on real-valued DMD fields, so it only only makes sense to view these modes.

In figure 4.15, we can interpret positive values as rises in heat transfer relative to previous modes, negative values as drops, and zero values as nearly no change. Modes 7, 17, and 25 show interesting positive spikes. Mode 13, identified previously as a low spike, also shows here as a negative value due to its drop in heat transfer. These three mode shapes $(7,17,25)$ will also be used as test cases for morphing due to these characteristics.

We can visualize the heat transfer changes between these modes by calculating the difference in Nusselt number ratio from the reconstructed fields. These are plotted in figures 4.17, 4.18, and 4.19 for mode differences $7-5,17-15$, and $25-23$, respectively. These figures are split into five 'panels', which show the Nusselt number ratio difference for each of the five lower walls of the DMD subdomain. For easy visualization of what this means, refer to figure 4.16. Since the ribs are at an angle, this method does give some distortion to the real fields, but it is more important to see the differences relative to important flow features.

As displayed from the line plot, mode 17 carries the greatest difference and thus the most red color. All three modes seem to lose some amount of heat transfer on the rear bottom wall, near the center (panel E). This corresponds to a location in the flow just where the trailing edge vortex has de- 
parted from the rib, causing a disturbance in the vortex recirculating in the rib's wake. Mode 17's plot shows a great increase in heat transfer at the center of the rib on panels B and C, whereas in 7 and 25, this area has much subtler features. It stands to reason that the structures climbing over the rib in this location has a large spectral signature that registers in mode 17. Almost all of the rib top edges have a vertical striped pattern due to the shape of the modes, which are displayed in section 4.3.1. The striped pattern would result from periodic temperature structures moving across the rib, as might be expected from previous analysis on that location of the rib.

Figures which have the heat transfer split based on the surfaces defined in figure 4.16 are available in figures A.10 and A.11.

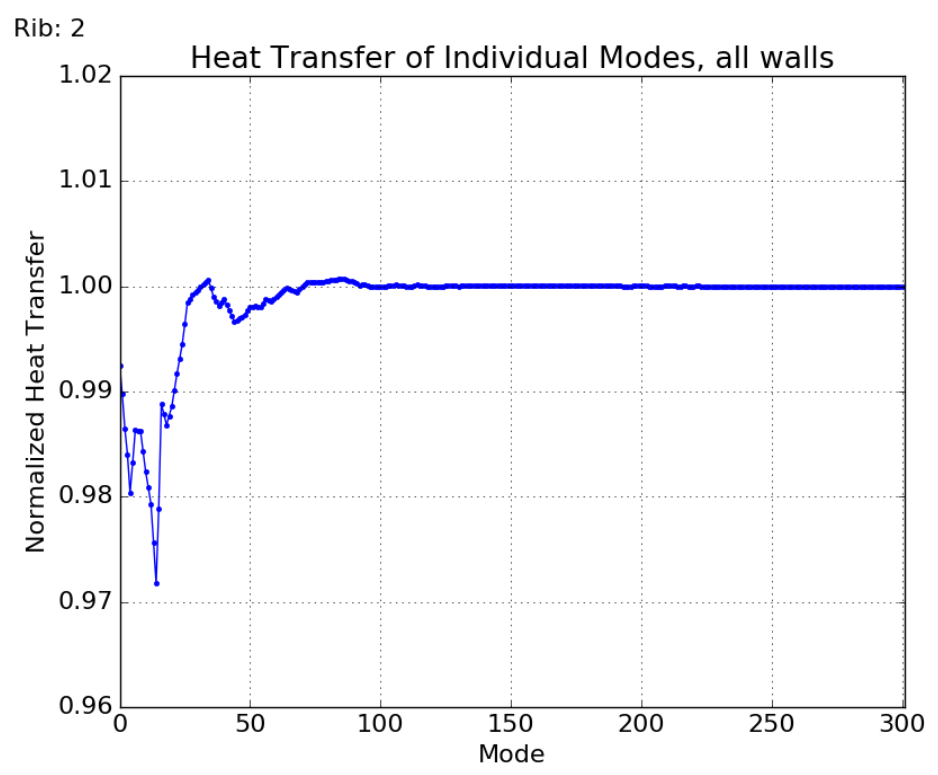

Figure 4.13: Normalized heat transfer for each DMD mode 


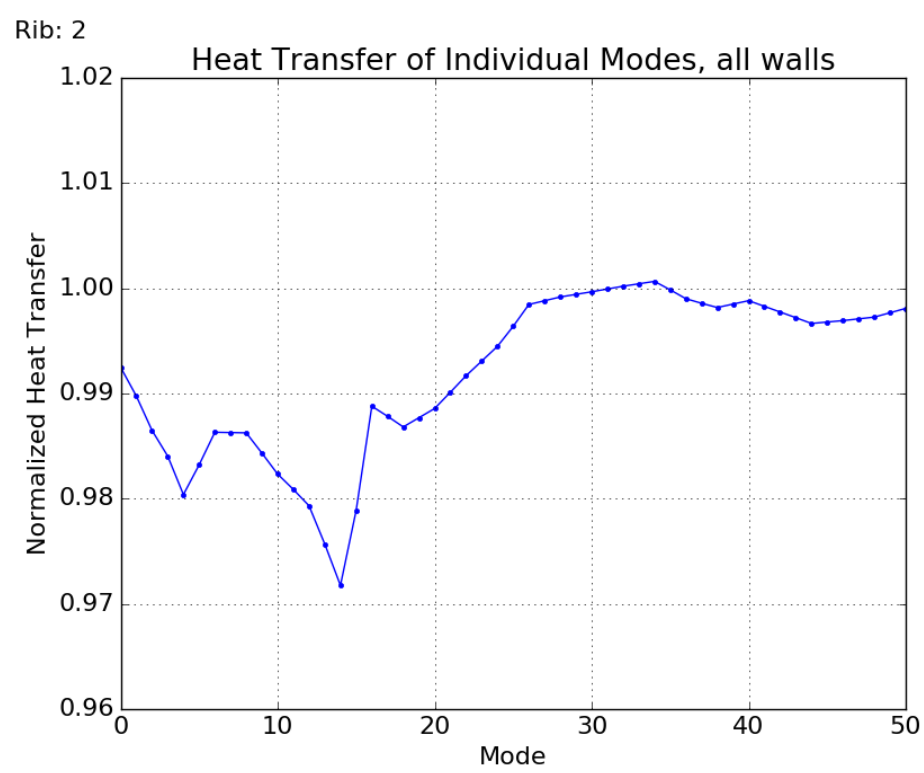

Figure 4.14: Normalized heat transfer for each DMD mode. Identical to figure 4.13, except for the $\mathrm{x}$-axis scale.

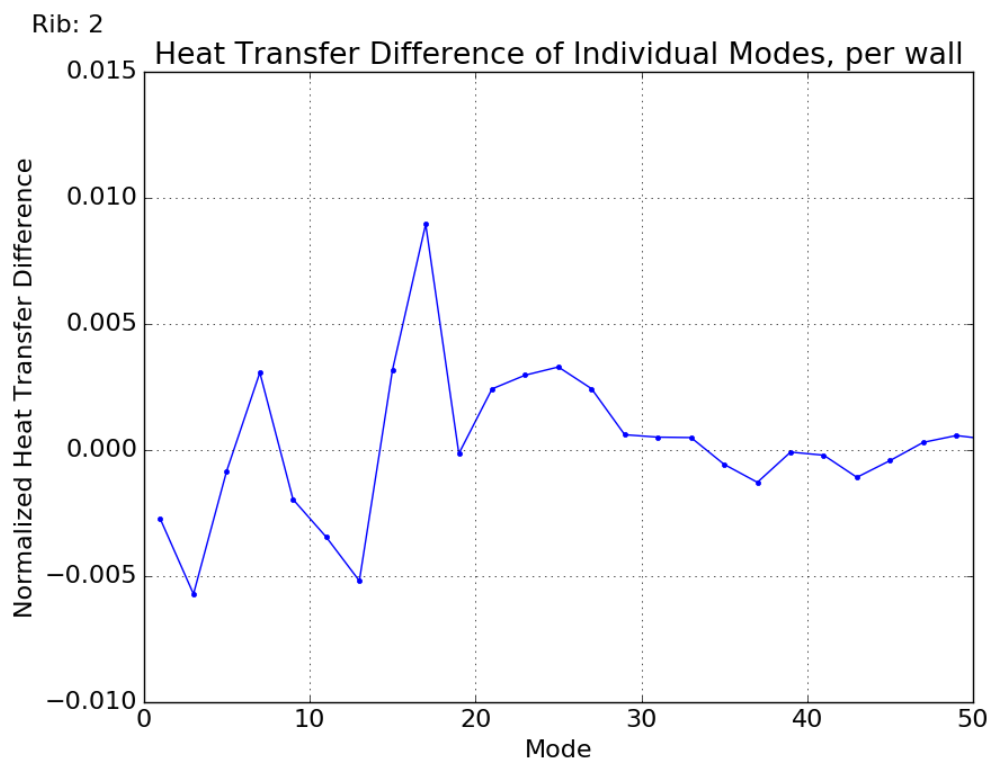

Figure 4.15: Difference in normalize heat transfer for each DMD mode. 

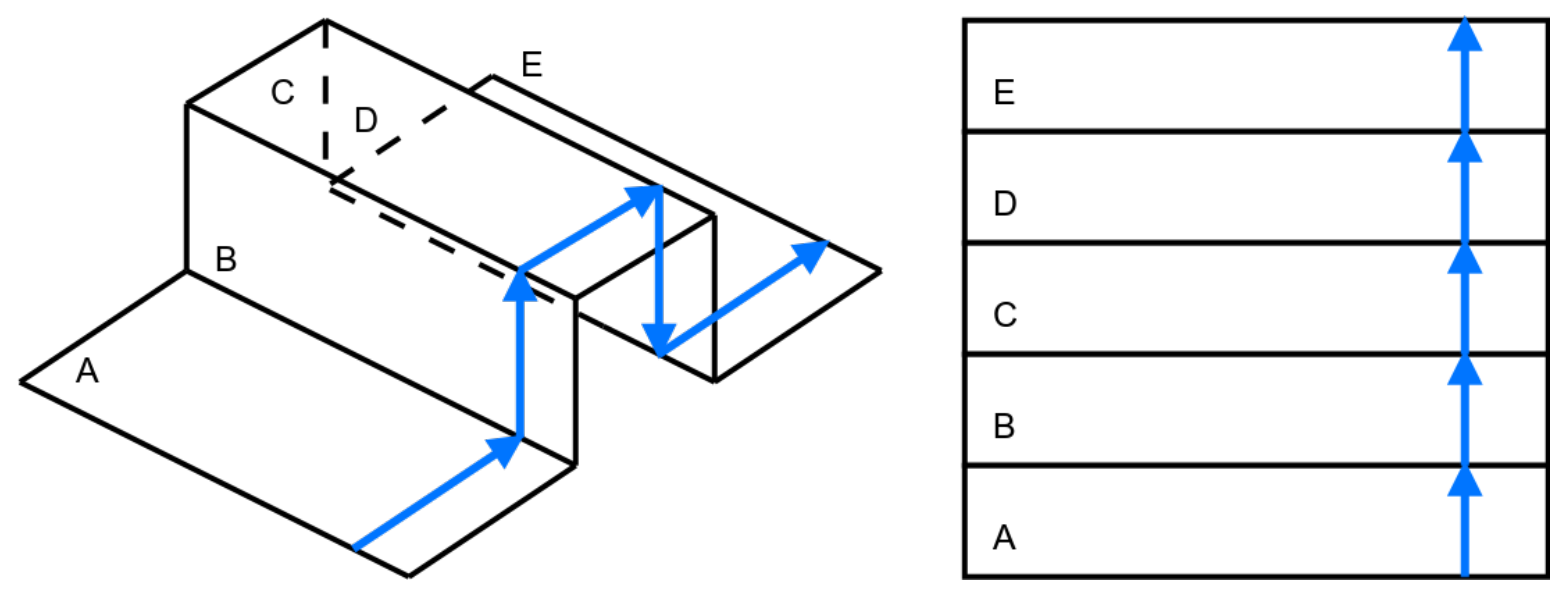

Figure 4.16: This diagram shows how the rib 'panels' are decomposed in the plots showing the reconstructed Nusselt number differences and DMD values near the surface. The blue arrow follows the main flow direction, making panel A the bottom of the channel in front of the rib. The surfaces are shown to be equally sized, but this is not the case in reality.

Rib: 2 Reconstructed Nusselt Ratio Difference, Modes: 007 - 005

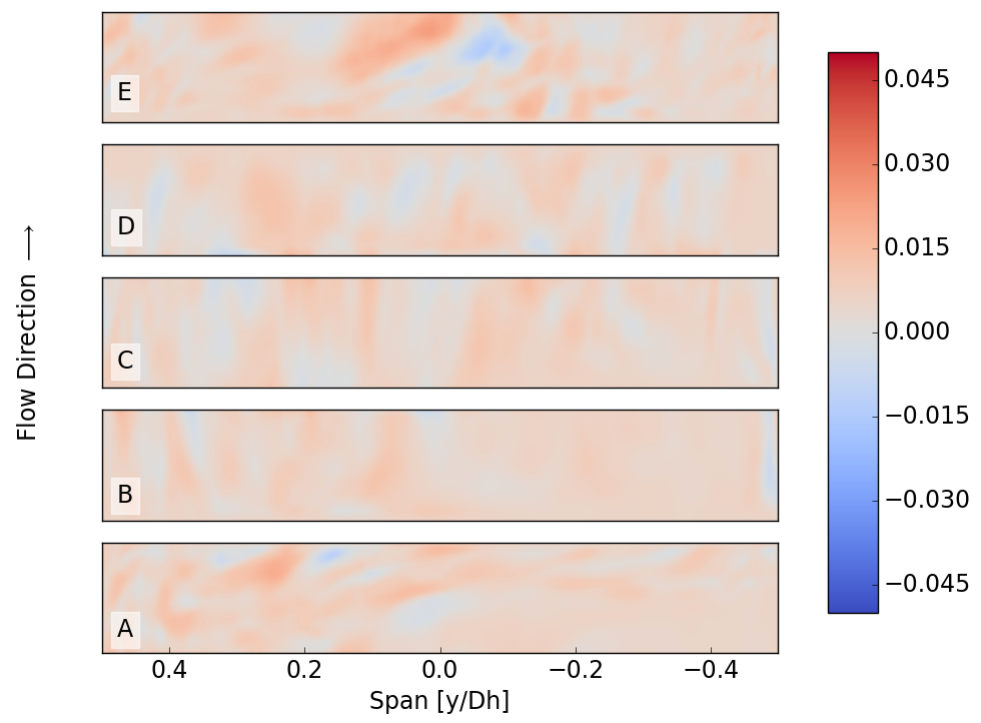

Figure 4.17: Difference in Reconstructed Nusselt Ratio from mode 7 to mode 5 
Rib: 2 Reconstructed Nusselt Ratio Difference, Modes: 017 - 015

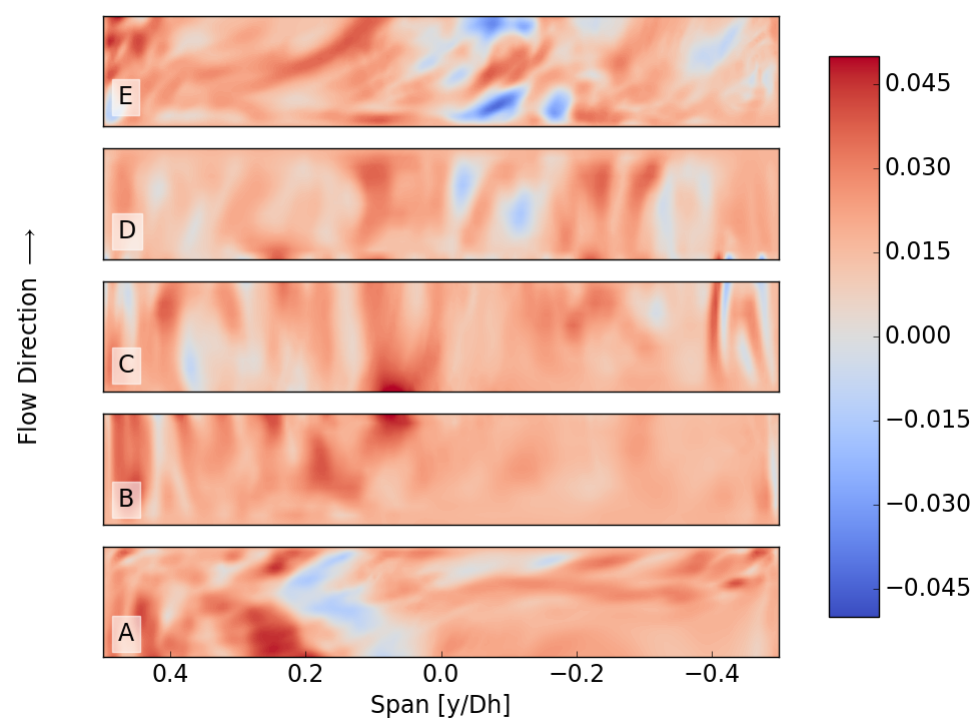

Figure 4.18: Difference in Reconstructed Nusselt Ratio from mode 17 to mode 15

Rib: 2 Reconstructed Nusselt Ratio Difference, Modes: 025 - 023

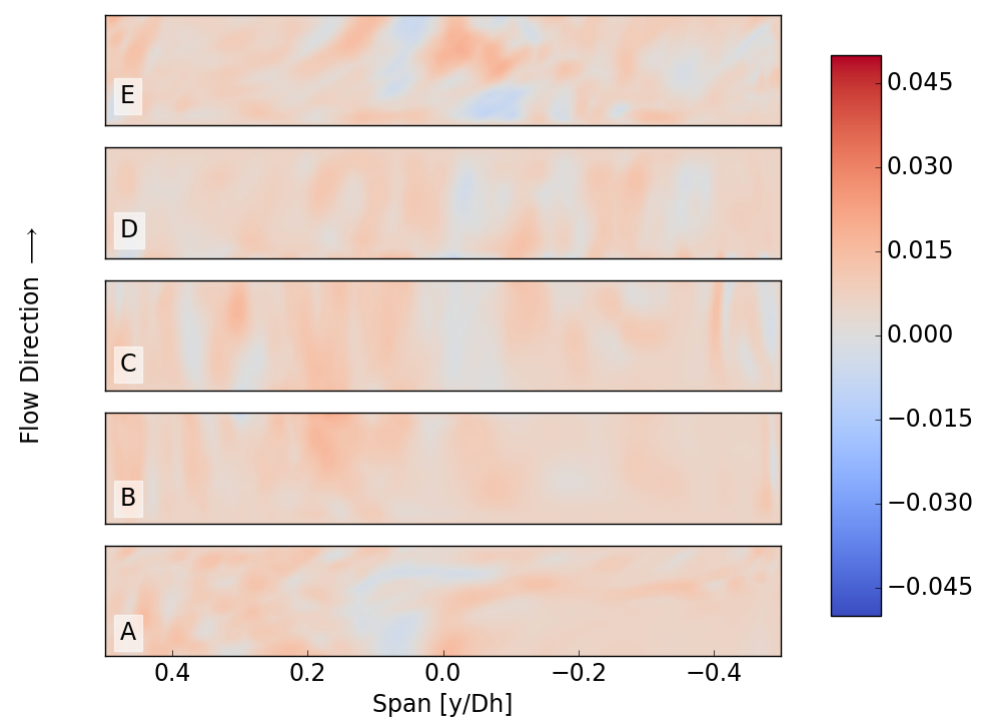

Figure 4.19: Difference in Reconstructed Nusselt Ratio from mode 25 to mode 23 


\subsubsection{Modes Selected for Morphing}

Following the analysis of the DMD modes' effect on heat transfer, six different modes were selected to use as inputs for modified geometries. A summary of the modes selected and the reasoning behind their choice is presented in table 4.1. The six different cases are also given designations for further work as D1 through D6. The six surface modes can be found in figures 4.20 through 4.25. Three-dimensional renderings of the mode shapes can be found starting with figure A.13. These surface modes are used as an input to the morphing stage. Indeed many of the large values in the DMD modes are spatially correlated with areas of major flow features. Areas where the flow carries more stability (lack of temporal change) show lower values of the DMD mode. In particular, the area just inside of $y / D_{h}=-0.5$ to about $y / D_{h}=-0.2$ is generally void of large characteristics for many areas of each mode. This area of the rib forms the large vortices that play larger roles elsewhere in the geometry and are relatively void of more randomized features. The exception is at the very edge of the rib $y / D_{h}=-0.5$, where a large vortex from the corner of the domain crawls over the rib, visible in figure 4.27. All modes contain a burst of content near the middle top of the rib, which corresponds to where the leading edge vortex finally blows over the rib. The vortex caused by the stagnation region of the rib shows as a braided feature in panel A of nearly every mode, and a striped pattern in panel B of nearly every mode. The mode structures are generally smoother and larger for lower ranked modes, easily visible when comparing, for exam-

ple, mode 17 and mode 99. Mode 7 seems to break this trend, which is explained in section 4.3.2. Recall that the modes are ordered based on amplitude, not frequency. Therefore if a high-frequency mode contains a very high-amplitude structure, it can easily appear as a low number mode. 


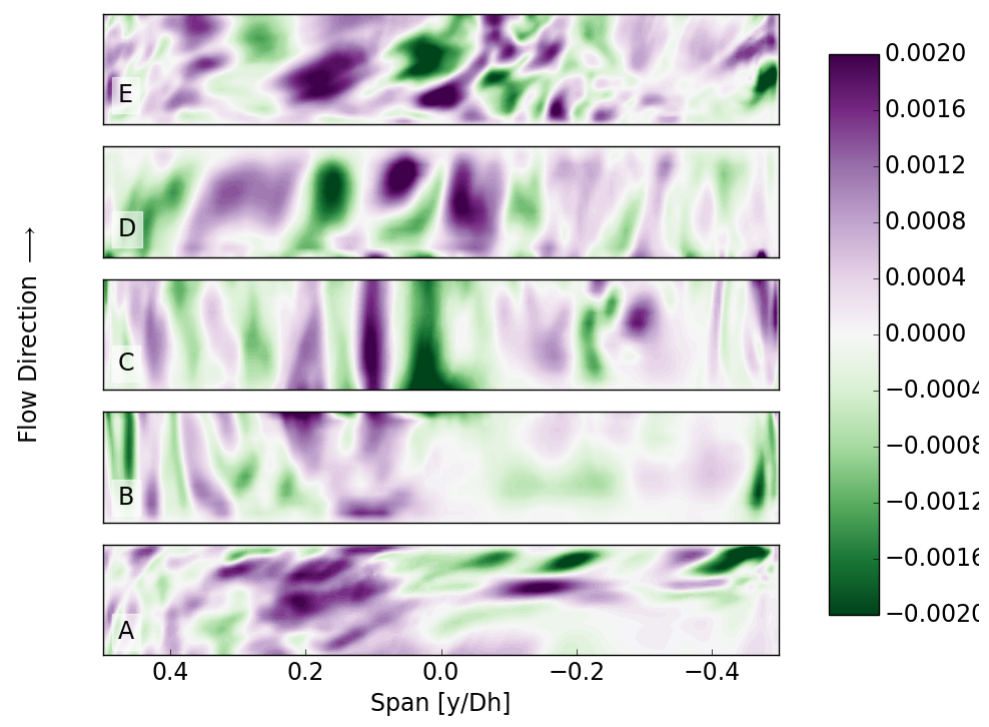

Figure 4.20: Near wall DMD mode shape, mode 13, case D1

Rib: 2

DMD mode 33 value near surface

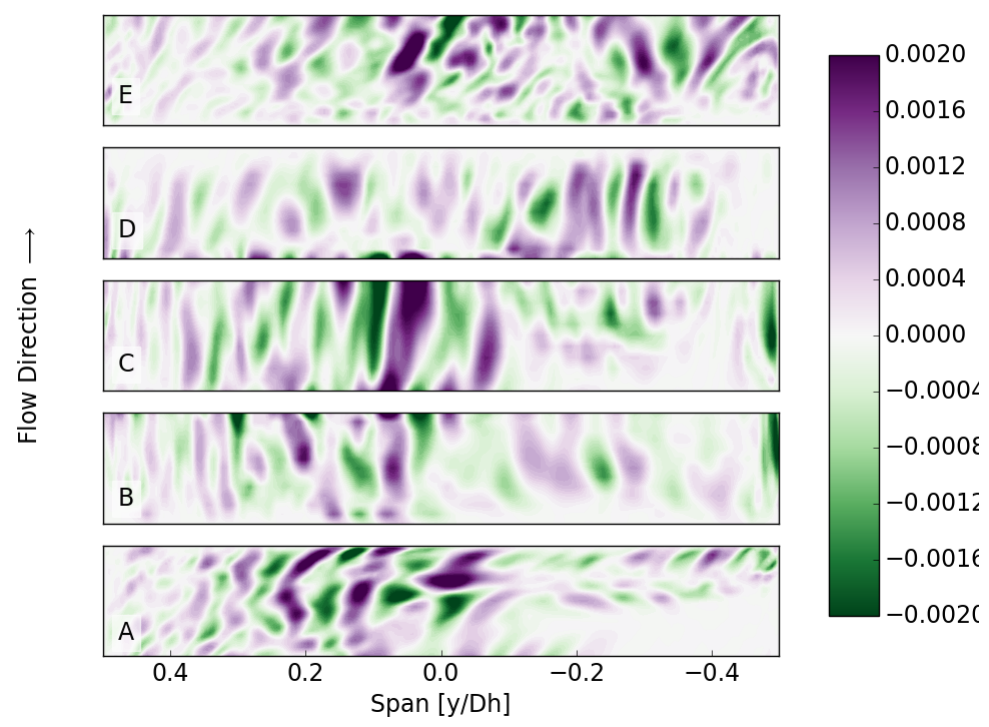

Figure 4.21: Near wall DMD mode shape, mode 33, case D2 

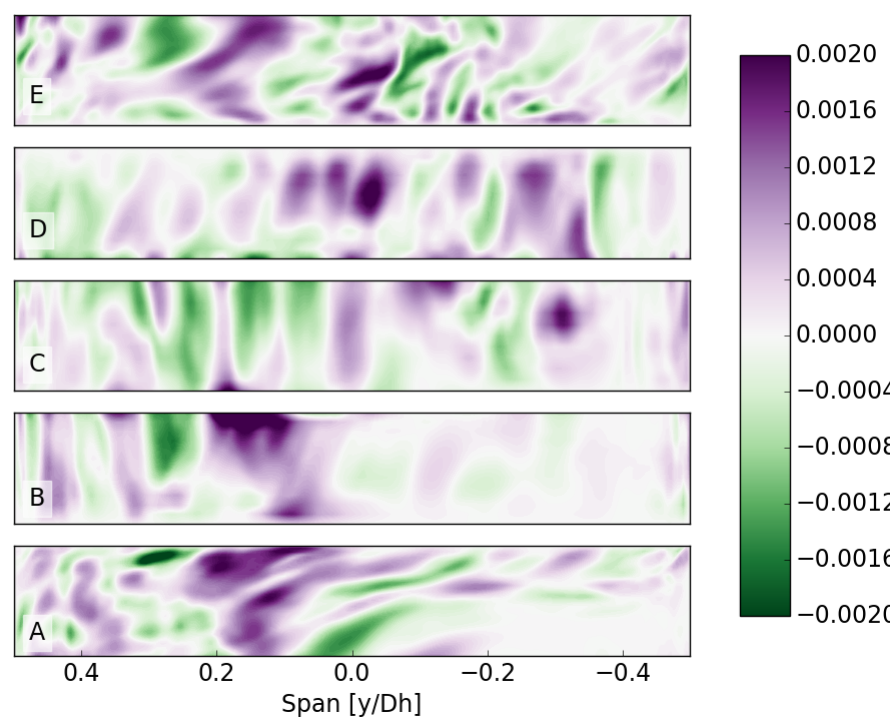

Figure 4.22: Near wall DMD mode shape, mode 17, case D3

Rib: 2

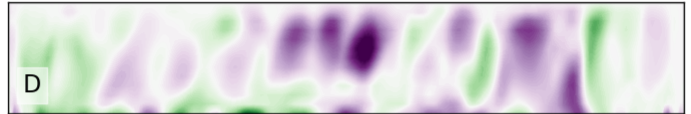

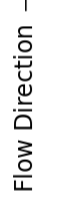
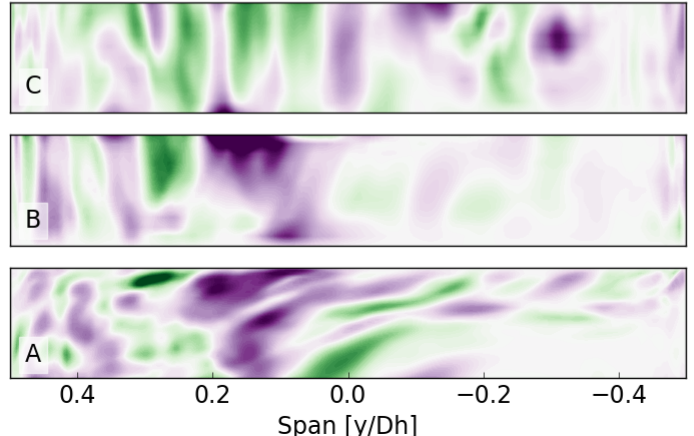

$.002 c$

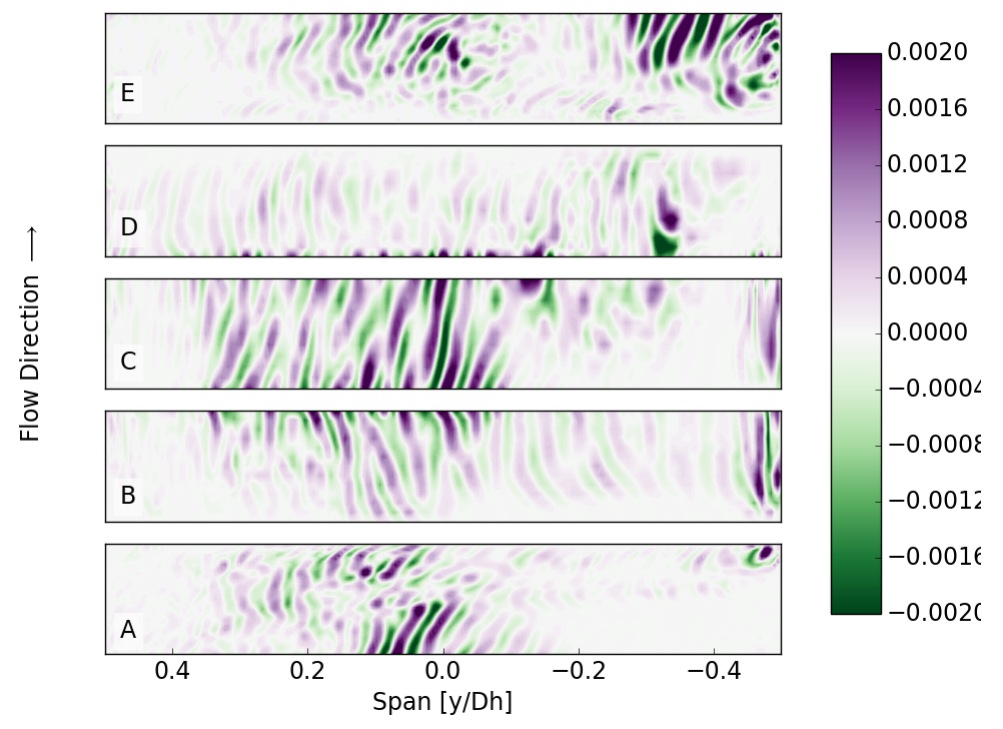

Figure 4.23: Near wall DMD mode shape, mode 99, case D4 


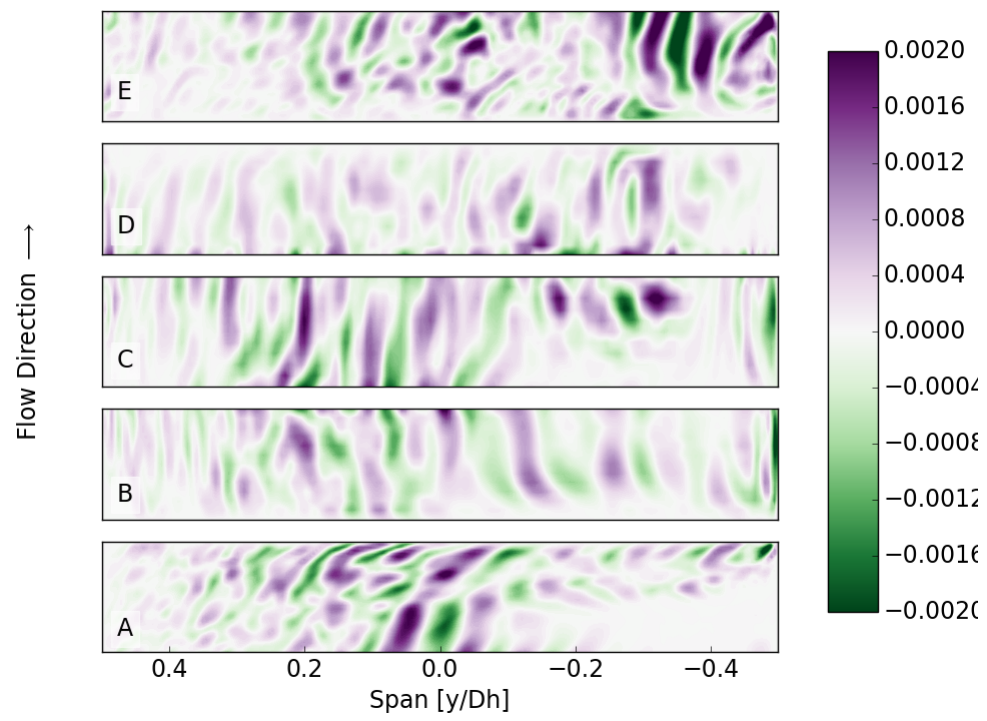

Figure 4.24: Near wall DMD mode shape, mode 7, case D5

Rib: 2

DMD mode 25 value near surface

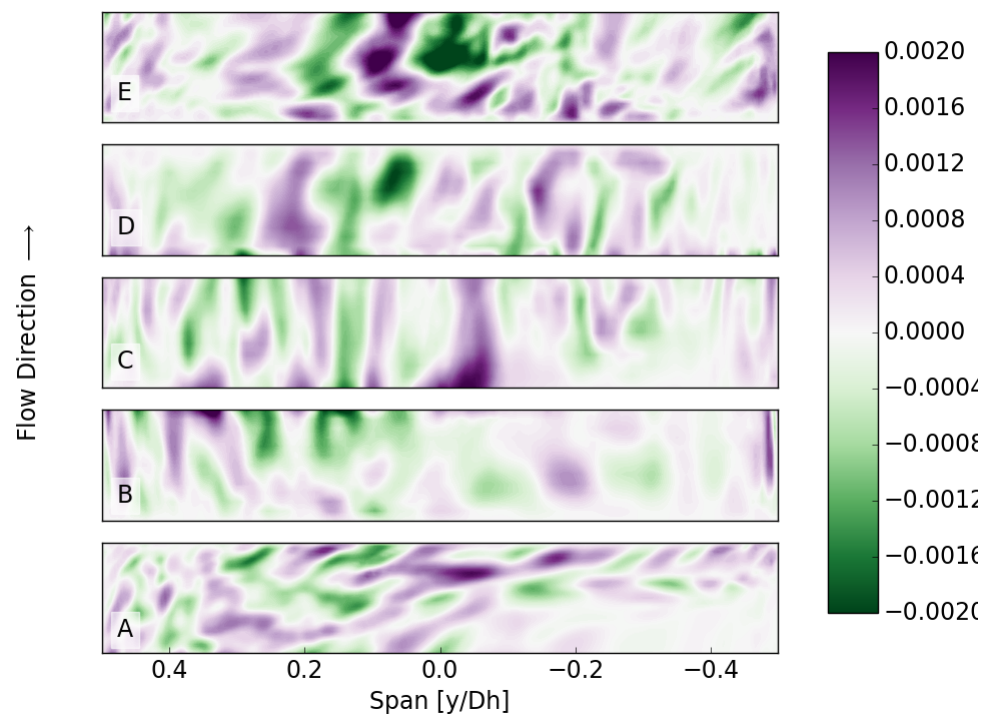

Figure 4.25: Near wall DMD mode shape, mode 25, case D6 
Table 4.1: Selected DMD modes for morphing and reasoning behind their selection

\begin{tabular}{l|r|r|l} 
Case & Mode number & mode shape figure & Selection reason \\
\hline D1 & 13 & 4.20 & Gives lowest predicted heat transfer \\
D2 & 33 & 4.21 & Gives highest predicted heat transfer \\
D3 & 17 & 4.22 & Gives highest rise in predicted heat transfer \\
D4 & 99 & 4.23 & Uninteresting mode, run as a check against other cases \\
D5 & 7 & 4.24 & Gives 3rd highest rise in heat transfer \\
D6 & 25 & 4.25 & Gives 2nd highest rise in heat transfer
\end{tabular}

\subsubsection{Spectral Comparison}

The DMD spectrum and selected modes are analyzed for any interesting qualities in this section. The spectrum from the analysis can be viewed in Figure 4.26. Recall that the DMD modes are ordered from highest amplitude to lowest amplitude, and therefore the first mode will appear as the highest point on the plot. In figure 4.26, the mean amplitude is not shown, as it is considerably higher than every other point. The six tested modes are also outlined in squares. Their frequencies and Strouhal numbers are displayed in table 4.2. The Strouhal number length scale used is the rib height, $e$.

Case D5 shows a large amplitude compared to its peers in frequency, which has a Strouhal number of 0.355 . This is slightly less than double the rib shedding frequency reported in literature. While there are point probes in the domain, their spectra do not show anything of note at this frequency. However at this part of the domain, the point probes are quite far from the rib's shear layer, so they may not be able to capture any interesting feature. There are some clues in the 3D mode shapes, however. In reviewing the shapes preceding mode 7 (case D5's mode), there are two differences seen when transitioning to mode 7 . One being the structure at the leading edge of the rib, the second being the structure about $3 / 4$ of the way down the sweep. Since mode 7 is the first to show these large structures, it stands to reason the amplitude anomaly many be due to one of these fea- 
tures. They appear to be the largest and most identifiable structures inside the DMD domain. Other structures exist near the end of the rib sweep, but that location is quite noisy compared to these two structures further up the rib. The structures' locations far from the center also would explain why none of the point probes show their presence, being situated in the center themselves. These structures and plots showing features of them in instantaneous LES images are visible in figure 4.27.

Concerning the other modes, they all exist in a fairly low frequency range, with D4 being the chosen exception. Modes 13 and 25 may be harmonics, but upon review of their mode shapes resemblance is minor.

Table 4.2: Frequencies of the six modes used for morphing.

\begin{tabular}{l|r|r|r|r|r|r} 
& D1 & D2 & D3 & D4 & D5 & D6 \\
\hline Frequency [Hz] & 171.2 & 873.8 & 217.2 & 2427.6 & 1136.7 & 347.2 \\
Strouhal number & 0.053 & 0.273 & 0.068 & 0.759 & 0.355 & 0.108
\end{tabular}




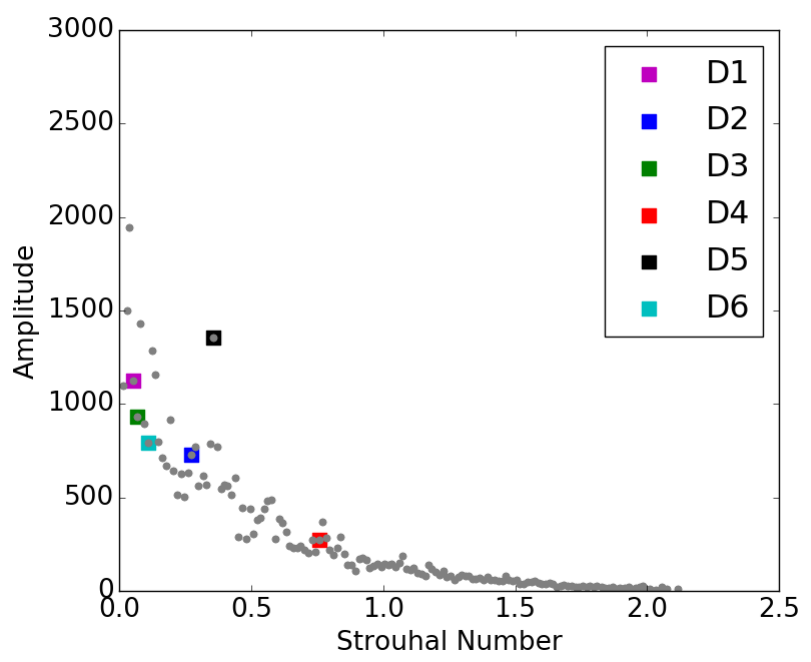

Figure 4.26: DMD spectrum plot. The six modes used for morphing are marked with squares. 


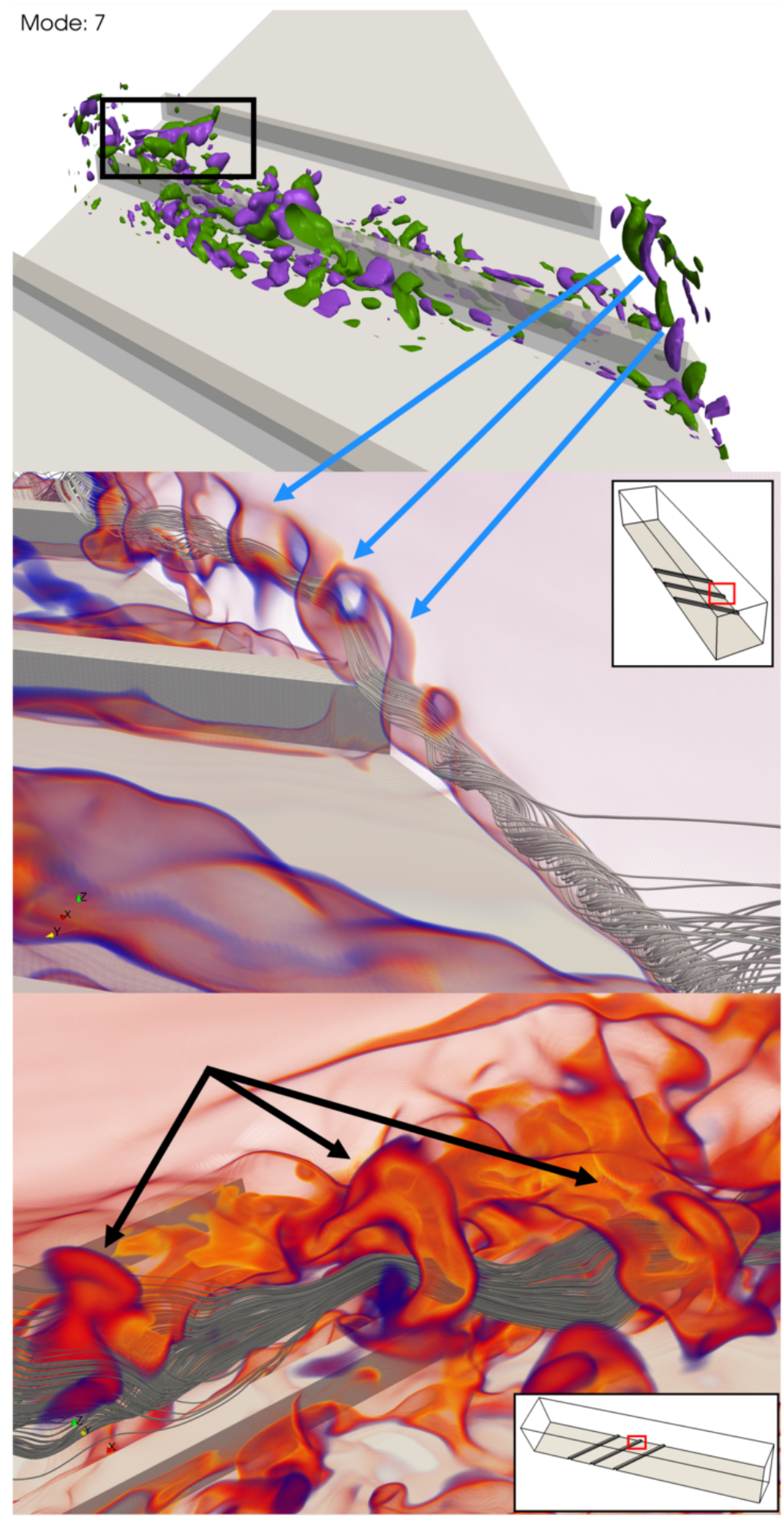

Figure 4.27: Noteable structures possibly causing the amplitude spike in mode 7 visualized in instantaneous temperature fields. Graphics with red rectangles indicate the location the camera aims at. 


\subsection{Modified RANS Case Geometry}

From the output of the DMD, the 6 different meshes are constructed by morphing the original volume mesh with a displacement at the surface. Morphing the volume mesh allows easy transition between cases with a mesh that has nearly identical refinement, but presents robustness challenges due to the highly constrained movement from the cell connectivity. Calculation of the displacement field involves 2 steps. First, the raw DMD field $\phi_{k}$ of the desired mode $k$ is scaled proportionally by a factor to get the values to a scale which is geometrically feasible. Second, the scaled DMD field is filtered to remove displacement in critical areas which cause morphing problems. These filters are located at the extreme ends of the span, and at the corners where the rib meets the bottom duct surface. The filter sets the displacement at these locations to zero, dramatically improving robustness. It was found that most robustness problems occur along the leading and trailing top corners of the rib. This is an area of high gradients in almost every field, making large values for the DMD shape possible. Oftentimes this area was the determining factor for the chosen scale factor, which is chosen just large enough to avoid negative volume cells and maintain good mesh quality. The scaling factors for each case, along with the maximum and average surface displacement, are noted in table 4.3. The deformations (denoted $d$ ) reach their maximum generally at a ratio $d / e=0.035$. All of them are relatively similar, with the exception of D3, which has some extreme values on the rib top forcing lower values. A plot containing the displacements of each case across the span can be found in figure 4.28. Further deformation becomes quite difficult without creating negative volume cells. This could be alleviated by extracting the surface geometry and meshing it in an unstructured manner, but this was not performed here to preserve a similar mesh strategy between cases.

By using the average deformation, some insight can be gained to understanding the deformation magnitude from the roughness standpoint. Theory states if the roughness height is smaller than the height of the viscous sublayer, then the flow above the viscous sublayer is unaffected by the 
surface roughness. This would occur if the roughness height $k$ combined with the viscous length scale obeys:

$$
\frac{u_{f} k}{\nu}=k^{+}<5
$$

From the information in table 4.3, the average displacement is quite small, which for most cases causes $k^{+} \leq 0.5$. This means the roughness is almost always entirely confined to the viscous sublayer, indicating a 'smooth' surface to the flow outside. There are local spots where the displacement may overpower the viscous sublayer, as can be seen by the scale of the maximum displacements, so it may be stated that locally there may be areas of roughness. It should also be understood that the 'dents' in the surface formed from morphing are generally anisotropic; they are shallow in the wall-normal direction, but often cover a larger area in the tangential direction, which allows them to affect the flow above them more than might be expected. A more traditional view of roughness would be taken as nearly isotropic deformations, like sand-grain roughness. Considering these facts it is unlikely that viewing results from the rough standpoint will yield useful information.

Table 4.3: DMD field scale factors and displacement $(d)$ statistics for morphing.

\begin{tabular}{l|r|r|r|r|r|r} 
Case & Factor & Avg $d[\mathrm{~m}]$ & $\operatorname{Max} d[\mathrm{~m}]$ & $\operatorname{Avg} d / e$ & $\operatorname{Max} d / e$ & $\operatorname{Avg} k^{+}$ \\
\hline D1 & $33^{-1}$ & $2.03 \times 10^{-6}$ & $1.15 \times 10^{-4}$ & $6.40 \times 10^{-4}$ & $3.61 \times 10^{-2}$ & 0.031 \\
D2 & $30^{-1}$ & $1.88 \times 10^{-6}$ & $1.11 \times 10^{-4}$ & $5.93 \times 10^{-4}$ & $3.50 \times 10^{-2}$ & 0.028 \\
D3 & $47^{-1}$ & $9.63 \times 10^{-7}$ & $6.18 \times 10^{-6}$ & $3.03 \times 10^{-4}$ & $1.95 \times 10^{-3}$ & 0.015 \\
D4 & $24^{-1}$ & $1.37 \times 10^{-6}$ & $9.94 \times 10^{-5}$ & $4.30 \times 10^{-4}$ & $3.13 \times 10^{-2}$ & 0.021 \\
D5 & $25^{-1}$ & $1.89 \times 10^{-6}$ & $1.13 \times 10^{-4}$ & $5.94 \times 10^{-4}$ & $3.57 \times 10^{-2}$ & 0.028 \\
D6 & $26^{-1}$ & $2.11 \times 10^{-6}$ & $1.13 \times 10^{-4}$ & $6.65 \times 10^{-4}$ & $3.58 \times 10^{-2}$ & 0.032
\end{tabular}



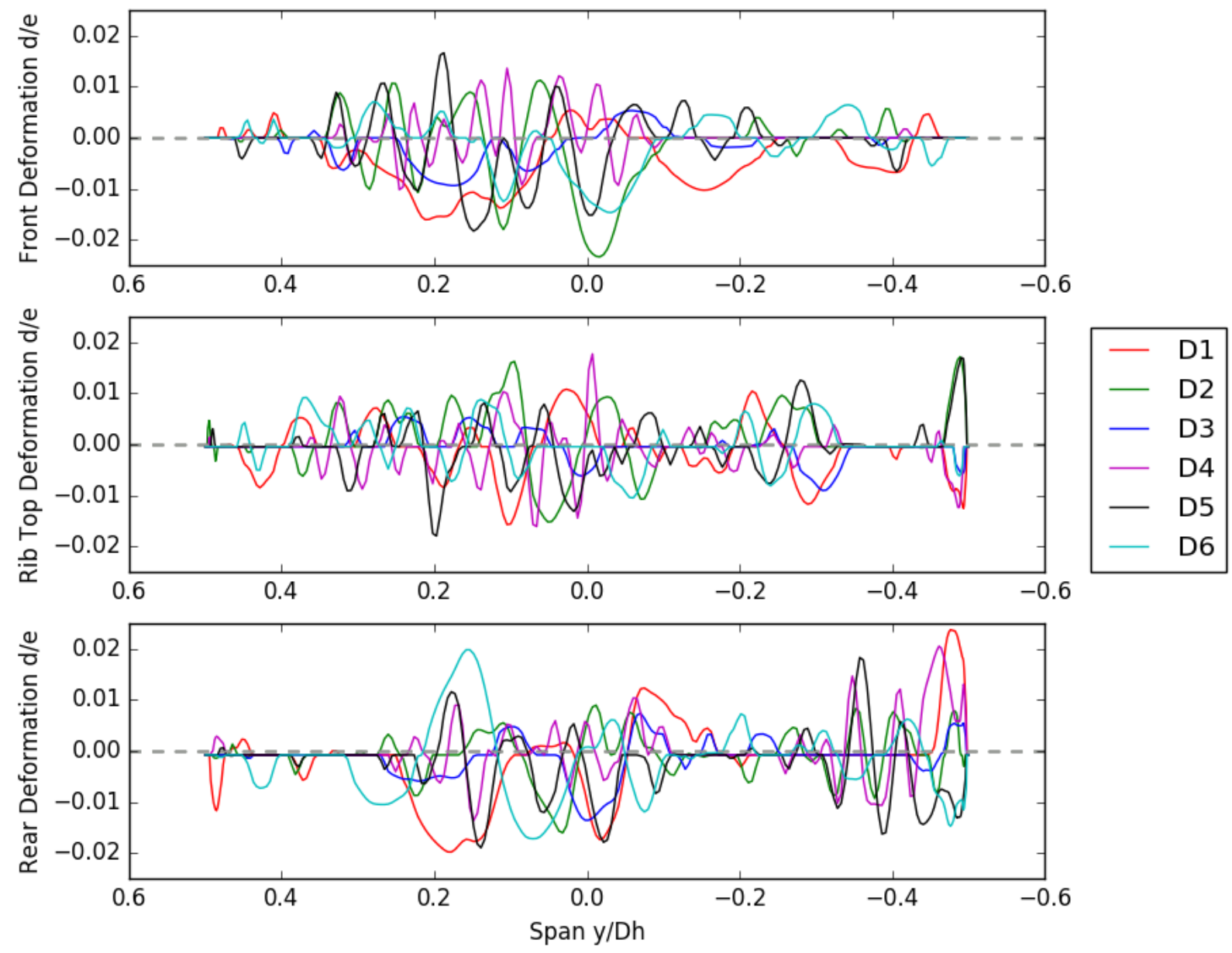

Figure 4.28: Shows the deformation $d$ of a line along the span of the bottom duct wall through the area in front, behind, and on top, of the rib. Deformation is normalized to the rib height, $e$. 


\subsection{Modified RANS Case Results}

\subsubsection{Pressure Drop}

The pressure loss forms the 'check' on the heat transfer values, as they are so often competing. Table 4.4 shows the darcy friction factors for all cases here, and the RANS cases are evaluated with respect to the baseline case. All show a lower friction factor. Since the magnitudes of surface displacements in this case are quite small relative to the duct diameter, these losses are likely more realized by changes in the flow physics rather than having a wider duct.

Table 4.4: Comparison of friction factor for morphed cases. The LES case is provided as a reference.

\begin{tabular}{l|r|r|r|r|r|r|r|r} 
& LES & RANS baseline & D1 & D2 & D3 & D4 & D5 & D6 \\
\hline Friction Factor & 0.0611 & 0.0686 & 0.0683 & 0.0676 & 0.0682 & 0.0679 & 0.0677 & 0.0678 \\
\% change & -10.9 & 0.0 & -0.44 & -1.46 & -0.58 & -1.02 & -1.31 & -1.17
\end{tabular}

\subsubsection{Heat Transfer}

Table 4.5 shows the heat transfer values of all six cases expressed as a percent change from the baseline RANS case. The heat transfer is broken up into several values. The overall value represents the heat transfer from the entire bottom surface of the duct, including the ribs. The 'bottom' surface is then the bottom of the duct excluding the rib surfaces. Each rib is then broken up into front (leading side), top, and rear (trailing side) panels, similar to the plotting scheme done for the DMD mode shapes. Recall that rib 2 is the only rib which has morphing applied, the rest are held constant. These local relative heat transfer values can be quite large thanks to the relatively low 
absolute heat transfer values. This explains why the local heat transfer changes can be large, but the overall values are small.

The immediate result is the failure of all cases to improve heat transfer. Recall that case D4 was added with an uninteresting mode shape in order to give a pseudo control test case. It shows a heat transfer loss near the middle of the six cases, and larger losses (percent wise) on specific walls, especially on the rear of rib 2. Since this case should show little change according to theory, this indicates a problem either with the theory or with the methodology of proving the theory. The rear of rib 2 seems sensitive as well in cases D2 and D5. In nearly all morphed cases, rib 1 tends to perform about the same, but different than the baseline, indicating the mere presence of the morphing has applied a similar change in the flow to all cases. The presence of 3 results with large losses in heat transfer in the same locations indicates the disturbance of a major flow feature due to the morphing in some kind of bifurcated way. This is explored in section 4.5.3.

The "best" performing case is D3, which showed the largest rise in heat transfer from the modal analysis. It shows an increase in heat transfer on the rear of the rib, the only case to show an increase of any kind on the morphed ribs, and even shows small increases on rib 3. While rib 2 does not show increases on the top, its top is one of the best performing, though its front is one of the worst. This provides some encouragement, since the mode for D3 was chosen since it gave the greatest increase in heat transfer relative to the mode previous to it.

While these results are disappointing, they do not necessarily indicate that the method of determining important heat transfer modes described here is nonfunctional. Two things may be hindering the results of the study.

First, the RANS cases may just not be capable of showing the changes in the flow necessary to give the behavior a scale-resolved simulation would. One turns to the differences between the RANS and LES simulations in friction and heat transfer, shown in previous sections. This difference will never be zero merely because of the large differences between the two methods. RANS simulations depend somewhat on resolving what one might call tonal structures; that is, large structures 
which are primary and secondary flow features. These structures should have some sensitivity to certain DMD modes, but without the rest of the spectrum available, may not be excited in the same manner that a scale-resolved simulation would show. This can be thought of from the RANS perspective as perturbing an average value in a dataset, whereas LES would be perturbing the data itself, and any modifications from the perturbation would be noticed upon taking the average. As stated previously this is an admittedly large issue with this study. RANS was chosen as a validation method merely because of the costs associated with running a second LES case. This second scale-resolved simulation would be the best way to show changes. This is perhaps why case D4, which was hypothesized to be uninteresting, instead shows relatively large changes compared to other cases. If case D4 had in fact shown little increases in heat transfer and other cases showed large losses, this may suggest the dominate mode identification methodology is incorrect. Since, however, D4 shows a difference, it is difficult to interpret the meaning of the results here with respect to the methodology's functionality.

Second, the mesh deformations may be too large or small. Recall that, due to mesh morphing constraints, case D3 has a lower deformation level than every other case, and it also shows the most promise in heat transfer results. One might argue if the perturbations designed to excite modes are too large, this is more akin to actually changing the starting geometry, and thus the resulting flowfield may have a completely different eigenmodes. One could also argue if the deformations are too small, resulting changes could be in the noise of the model, even if the convergence is excellent. More cases are being prepared to run with differing deformations on the same mode in order to determine if this is the case, but these simulations were not executed in time for writing. The only other author to perform wall contouring of any kind based on a mode shape was Schwanen in [50]. The deformations performed were of POD modes however, and those deformations were far larger in comparison to the work here. Schwanen had to make an argument that the heat transfer increase was due to the mode excitation rather than flowrate increases from geometry constriction. Since the deformations for this work are far smaller, it makes little sense to require such an argument 
here.

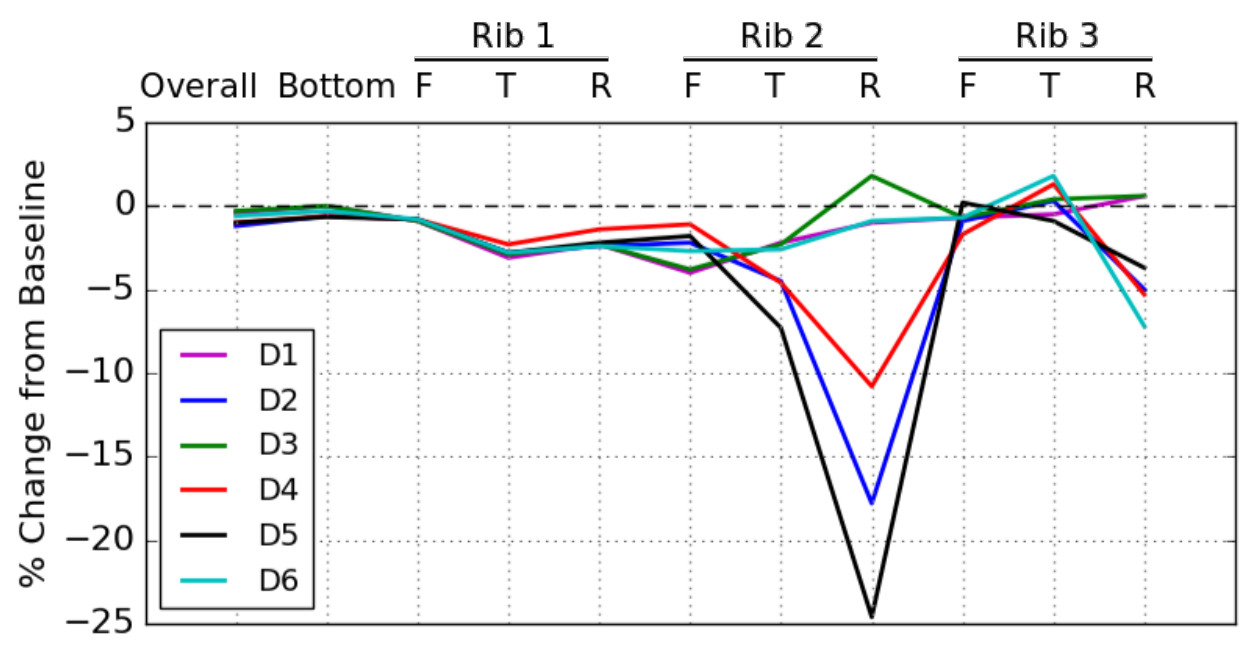

Figure 4.29: Percent change in heat transfer for different surfaces of the channel bottom relative to the baseline case. The bottom wall represents the surface without ribs on the bottom of the duct. $\mathrm{F}, \mathrm{T}$, and $\mathrm{R}$ represent the rib face front, top, and rear. 
Table 4.5: Percent change in heat transfer for different surfaces of the channel bottom relative to the baseline case. The bottom wall represents the surface without ribs on the bottom of the duct. Significant drops are in red, while any increases are in green.

\begin{tabular}{|c|r|r|r|r|r|r|r|r|r|r|r|}
\hline & & \multicolumn{3}{|c|}{ Rib 1 } & \multicolumn{3}{|c|}{ Rib 2 } & \multicolumn{3}{|c|}{ Rib 3 } & \\
\hline Case & Overall & Front & Top & Rear & Front & Top & Rear & Front & Top & Rear & Bottom \\
\hline D1 & -0.39 & -0.85 & -3.06 & -2.31 & -3.96 & -2.17 & -0.95 & -0.74 & -0.52 & 0.64 & -0.04 \\
D2 & -1.18 & -0.79 & -2.80 & -2.41 & -2.19 & -4.45 & -17.78 & -0.93 & 0.28 & -4.98 & -0.55 \\
D3 & -0.28 & -0.86 & -2.90 & -2.27 & -3.73 & -2.26 & 1.80 & -0.67 & 0.36 & 0.63 & -0.01 \\
D4 & -1.01 & -0.82 & -2.31 & -1.39 & -1.11 & -4.60 & -10.83 & -1.66 & 1.30 & -5.32 & -0.60 \\
D5 & -1.19 & -0.82 & -2.79 & -2.19 & -1.77 & -7.27 & -24.55 & 0.15 & -0.87 & -3.67 & -0.39 \\
D6 & -0.61 & -0.80 & -2.79 & -2.44 & -2.73 & -2.56 & -0.94 & -0.71 & 1.76 & -7.20 & -0.31 \\
\hline
\end{tabular}

\subsubsection{Flowfield Features and Description}

Post-processing identical to images shown for the LES and RANS case can be found starting with figure B.1. Their proximity to one another in the appendix should hopefully ease comparison. Many major flow features exist just as they did before, with slight modifications due to the morphing. The front of the rib showed smaller changes relative to the baseline, and is it difficult to see where those exist from the plots shown. The rear, top of the rib and bottom of the channel following it show larger changes that are easy to identify.

The cause of the heat transfer bifurcation between the six cases is of great interest. The three cases that show the lower heat transfer all appear to have a feature in their modes in common. Referring to the mode shape figures starting with figure 4.20, cases D2, D4, and D5 all share an increased DMD (absolute) value in the upper right corner of panel E. The other three modes lack this feature to a significant degree. This shape sits in the small gap between the pair of vortices behind the rib. The increased DMD values here lead to deformations that separate these vortices. The smaller inner vortex tends to move towards the rear of the rib, and upon view of the vorticity plots, appears to interact less with the wake vortex which occupies the space, resulting in less convective action. 
The large effect such small displacements of the wall had on critical flow structures may indicate further that the mesh deformation was too high. Viewing the Q-criterion plots for these six cases shows small structures forming in the bumps caused by the DMD deformations. These are best seen underneath the main trailing edge vortex. These bumps produce serrated patterns in Nusselt number ratio fields.

Also of interest are the reasonings behind the relative success of case D3. It showed a larger increase on the rear rib, which appears to originate from an increased interaction between the trailing structures, best viewed in the vorticity plot. The area of impingement on the top of the rib also appears longer than in other cases, which makes up for some of the relative loss on that part of the wall. 


\section{CHAPTER 5: CONCLUSIONS}

This paper explores the possibility of using Dynamic Mode Decomposition to improve a geometry based on an engineering quantity of interest, utilizing the same tools an industrial simulation engineer would have at his or her disposal.

To achieve this, several steps were executed. A steady-state baseline RANS case is used to give a starting simulation with which to compare all others. Following this case was an LES run of the same geometry. This run was mainly needed to generate the necessary time snapshots for the DMD analysis, but also offered the possibility of comparing the RANS case with a scale-resolved simulation. In comparison, the RANS case was able to predict the LES heat transfer with mild accuracy, though significant differences in flow structure from the first rib were found. These must be reviewed carefully, however, since the LES case at hand was not able to reach a statistically stationary state in time given computing resources at hand.

A new DMD code written in Python was used on the snapshots collected in the LES case. A convergence study was performed on the three parameters most affecting the DMD analysis: snapshot timestep, the amount of time snapshots are taken for (or implicitly the number of snapshots), and number of singular values kept after truncation. The convergence study showed that keeping singular values after about 100 gave diminishing returns. It also showed that a good combination of timestep and number of snapshots were necessary in order to attain good convergence, as opposed to having an extreme value in either category. Furthermore, the residual measurement against the primal field is most improved by lowering the timestep. For this case, the smallest timestep of $20 \mu \mathrm{s}$ and a long snapshot time of 5 to 10 flow-throughs of the DMD domain gave the best results. The DMD code was then used to extract information on the modes which may lead to an understanding their value toward heat transfer. Each mode was used to build a unique temperature field, from which heat transfer values were extracted. Analysis of the different heat transfer values was 
performed in 2 ways. One involves finding the partially reconstructed temperature field that most overpredicts or underpredicts the actual heat transfer, which might indicate some useful effect that mode is having. The second way involves finding a mode which most improved heat transfer relative the mode that came before it, suggesting the addition of that mode caused the change in heat transfer.

Once extracted, these modes' shapes were used to deform the surface of the channel in an effort to excite the modes. Six different shapes were extracted and used as an input for morphing. The morphed geometries were then simulated using a steady RANS technique identical to the baseline and compared. Comparison showed that all modes performed worse in heat transfer and slightly better in friction than the baseline case. One case, designated as a psuedo-control case with an uninteresting mode shape, showed a loss in heat transfer, suggesting that the RANS case may not be able to resolve the differences well enough for reliable answers. The case which performed the best was the case which had the highest increase in heat transfer relative to the previous mode. Since it also contained the lowest level of deformation, it may be possible the mesh deformations were too large, despite already being quite small compared to previous works. Further study will be required to validate these findings and ensure the methodology proposed for finding the most important modes is correct.

DMD is a relatively new algorithm and constantly evolving, as is the entire engineering simulation space. As parameter spaces for geometries become better understood, a more data-driven method is required to yield more performance on top of existing optimized designs. DMD, combined with a mode assessment methodology like the one proposed here, may give the engineer the ability to do just that. 


\subsection{Future Work}

The methodology proposed here requires additional study. The conclusions drawn from the heat transfer analyses bring forth ideas for future work.

\subsubsection{Sensitivity to Morphed Displacement Magnitude}

The first and foremost idea would be a study on the modified RANS cases for different morphing scale factors. If the morphed case performance can be greatly affected by lowering the amplitudes of the displacements, it may be true that the cases here were simply displaced too much. Since a previous work used a larger displacement, this may indicate the displacement is problemdependent. It may also be the case displacements were too small, though this seems less likely. In that case, considering the geometric constraints on volume mesh morphing, the best alternative would be to morph a surface and build a new unstructured mesh using that surface. This would allow far larger displacements than supported here. This work would then take two forms, described below.

The first part of this study would be performed on a morphed volume mesh, as done here. Several different scale factors for each chosen mode (or perhaps just one to start) would be used to produce several different meshes. These scale factors should range from a value which barely deflects the mesh to the maximum possible value before generating negative volume cells. All of these meshes would be computed with identical boundary conditions and then compared. There may be some medium point where the deflections are large enough to cause the desired change in the system, exciting modes, but also small enough to not cause major changes in large flow structures, as seen in the current work.

The second part of this study would be performed on an unstructured grid. The procedure is identical to the volume mesh morphing study described above, except for the grid generation component. 
Due to the heavily constrained nature of volume mesh morphing, an unstructured grid would allow even larger displacements than the volume mesh morphing cannot feasibly perform. The displacements would morph a surface mesh instead of a volume mesh, and that surface would then be used as an input to an unstructured volume mesh generator. Just like the volume mesh morphing study, a range of scale factors would be chosen from large to small and executed. It is recommended this study be performed second. If the volume mesh morphing tests show that large displacements do not show the desired behavior from RANS, then this test may be unnecessary. The only advantage of this methodology would be robustness to large displacements; if they are not needed, then this study is largely irrelevant.

\subsubsection{Verification of Results using LES}

Another future work would involve assessing whether RANS is capable of properly computing the changes a more realistic flow would see. This would be performed by taking the morphed cases and re-running them in LES. The following procedure would be a good starting framework.

- Develop a baseline heat transfer model with LES

- Perform DMD on sampled LES data

- Analyze the DMD data to identify modes which enhance heat transfer

- Modify the topology of the geometry to enhance the heat transfer from the mode shape information

- Perform an additional LES simulation

- Determine if an increase in heat transfer was found

- Determine if the DMD modes are similar to the previous cases' 
- Determine if the spectra are similar to the previous cases'

Particular attention must be paid to the mesh quality in this case, as LES is far less tolerant of poor quality cells than RANS. Because of this, adding upwind blending to the LES discretization may be necessary. One should make sure that both (baseline and morphed) cases use similar discretization, which might necessitate running some sample morphed cases (perhaps not to stationarity) before running the baseline case. Then the modal structures could be extracted from the morphed geometry and their spectra compared. If particular modes see increased amplitudes after the second computation and simultaneously show increases in heat transfer, this would be good evidence the methodology functions as described.

\subsubsection{Less Expensive DMD Data Simulations}

Another study which may be intriguing involves attempting to sidestep the obvious costs of LES. If a method like this is to be useful to the industrial engineer, building a single LES model may prove too costly. However, since DMD attempts to create low-rank structures, it could be argued that using a simulation which does not resolve as many turbulent scales would be sufficient. Such a simulation could be a RANS/LES hybrid like Detached-Eddy Simulation (DES). A comparison of mode shapes, especially low-rank ones, between DES and LES cases may prove very useful to industry, as DES, though expensive, is cheap enough for wide usage in many areas. If RANS is found incapable of showing mode shape changes and LES is too expensive, DES may provide the necessary tradeoff. The obvious drawback to this would be that DES uses RANS near the boundary layer. For heat transfer modeling, much of the activity revolves in that location. Performing a DES

case without the true backdrop of a comparison LES case may show mixed results as seen here. This study's procedure would be similar to the verification of LES: 
- Develop a baseline heat transfer model with LES

- Perform DMD on sampled LES data

- Analyze the DMD data to identify modes which enhance heat transfer

- Perform an additional DES/SAS/other method simulation

- Analyze the DMD data to identify modes which enhance heat transfer

- Compare the spectra and identified modeshapes from the two above. Similar shapes and outcomes may mean the cheaper simulation is sufficient. 


\section{APPENDIX A: ADDITIONAL FIGURES}



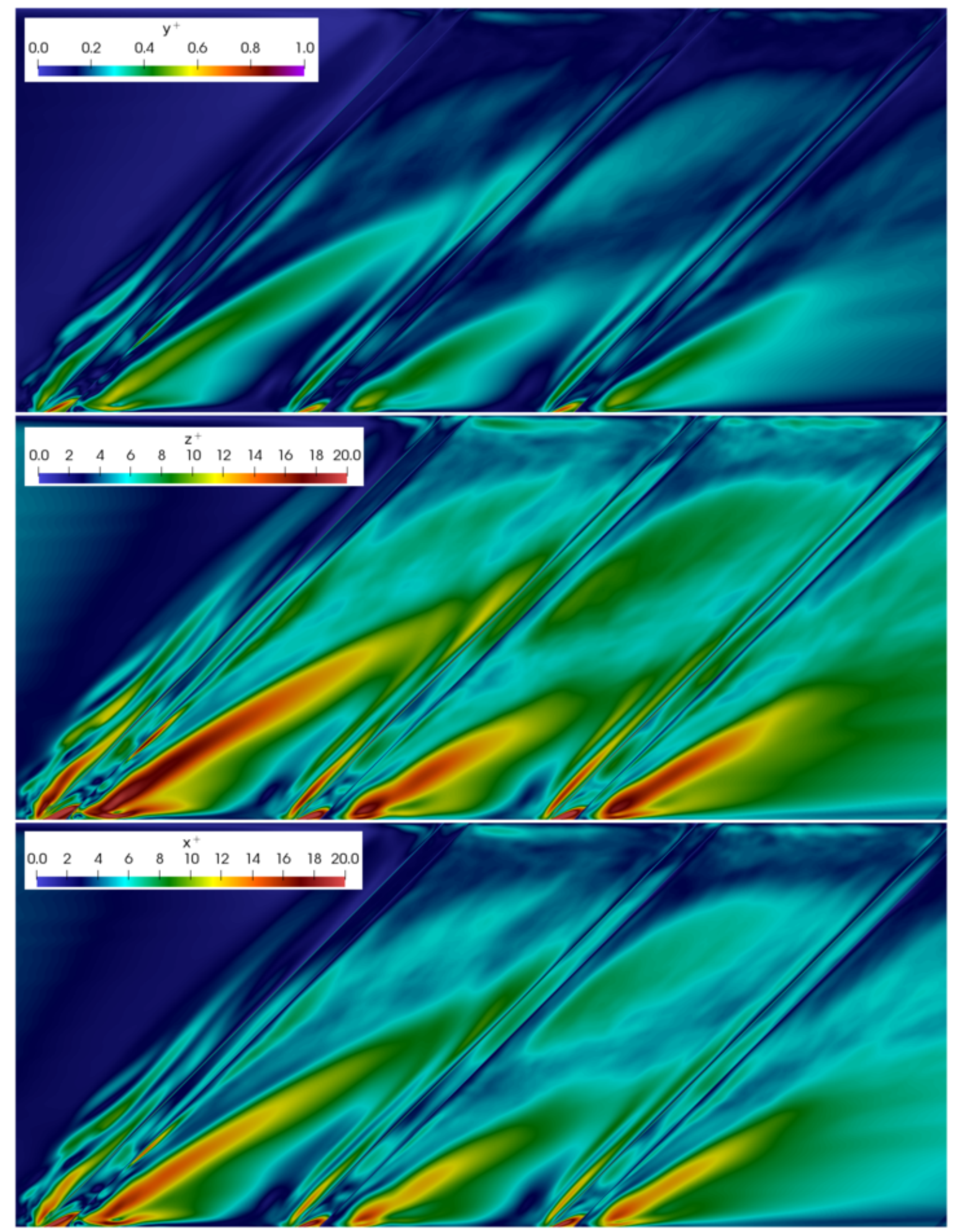

Figure A.1: $y^{+}$(wall-normal), $z^{+}$(spanwise), and $x^{+}$(streamwise) viscous wall spacing metrics. See section 3.6.5 


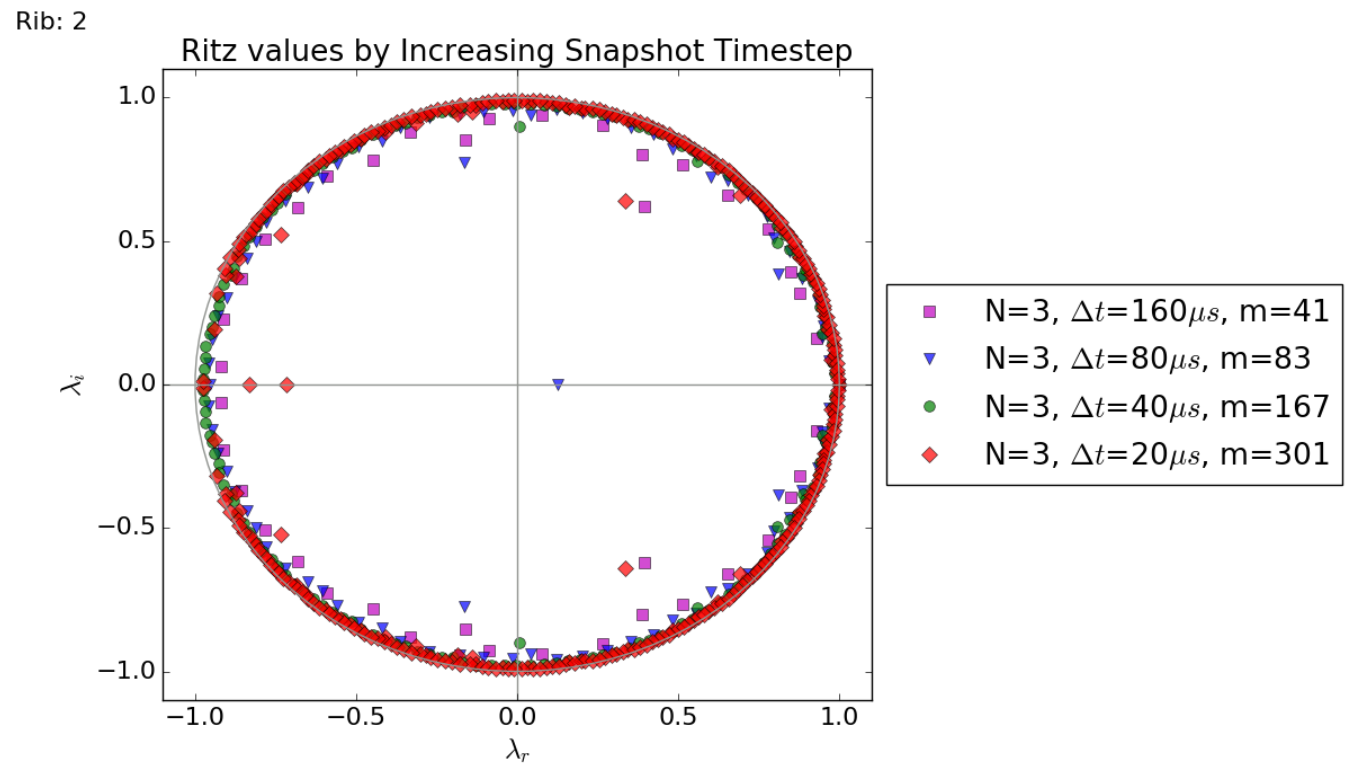

Figure A.2: Ritz values for $N=3$.

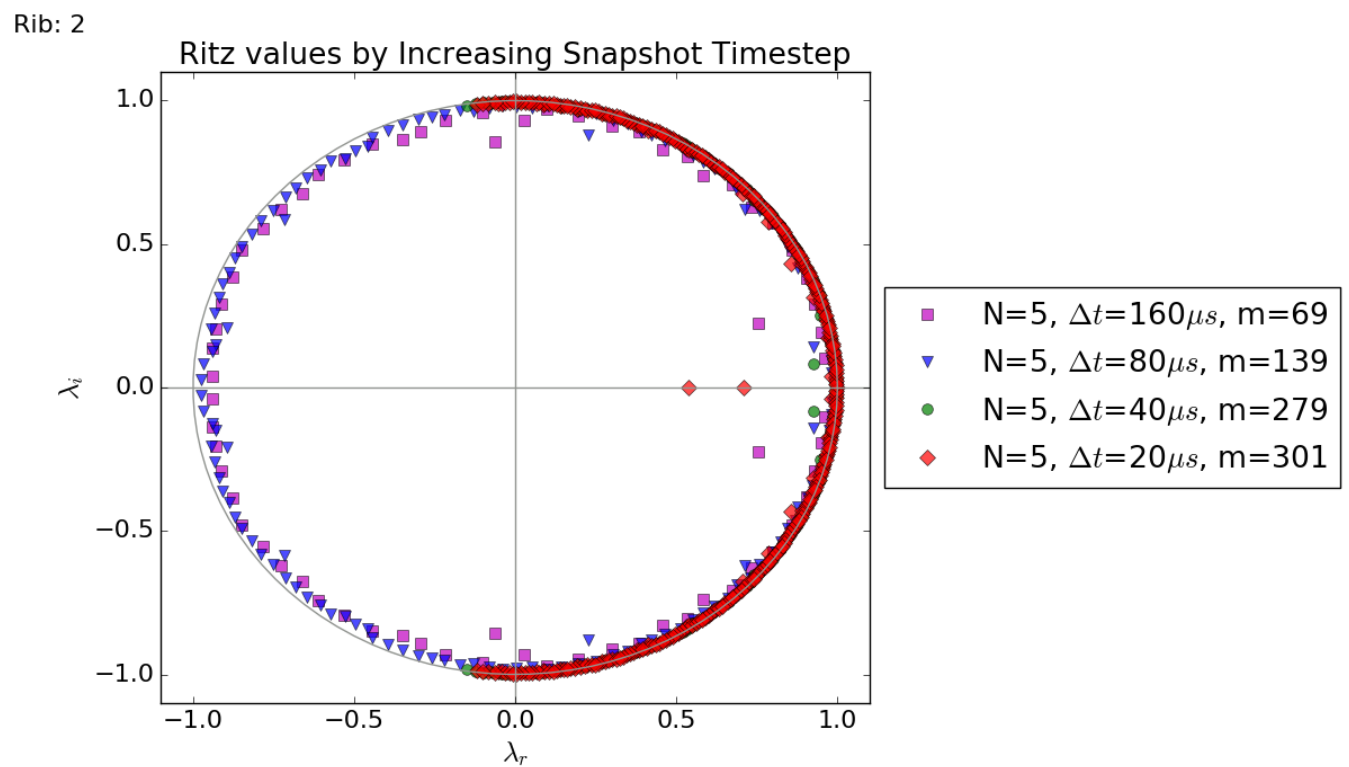

Figure A.3: Ritz values for $N=5$. 


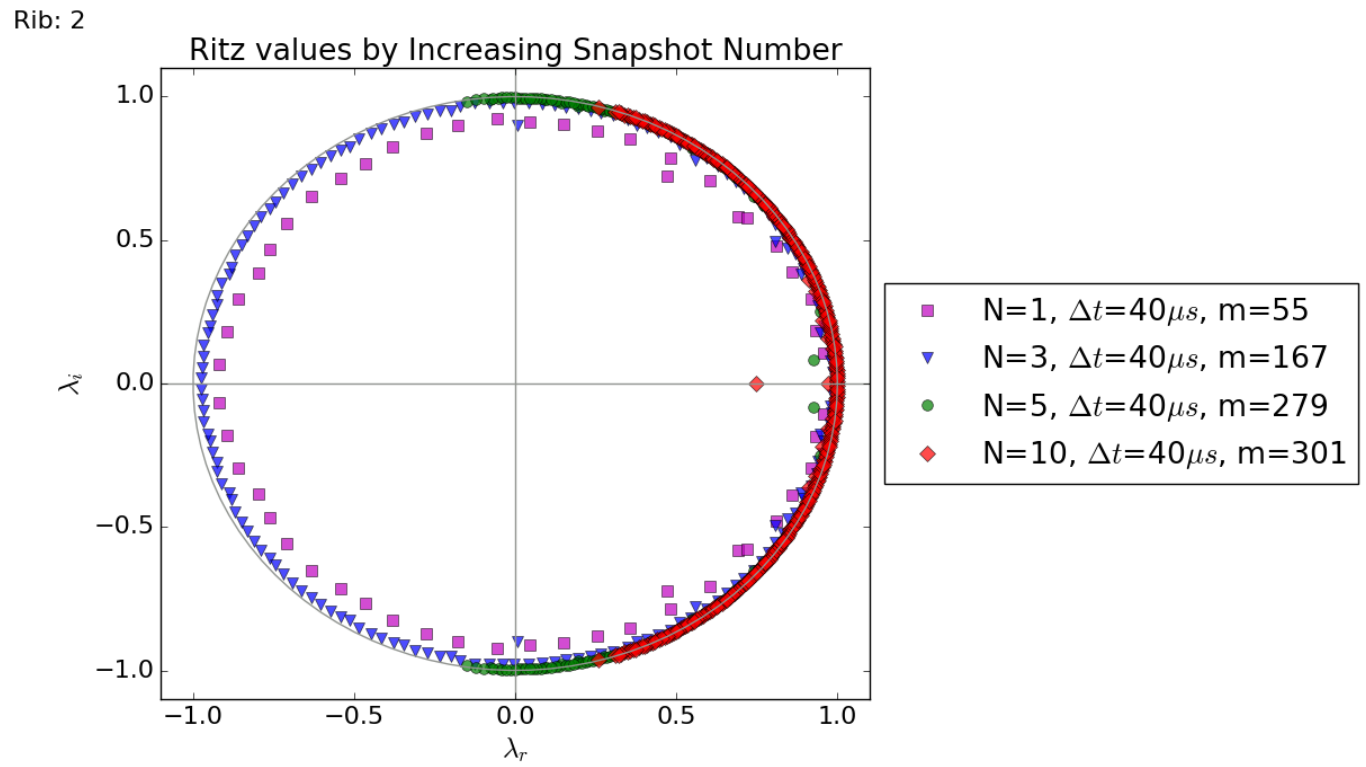

Figure A.4: Ritz values for $\Delta t=40 \mu s$.

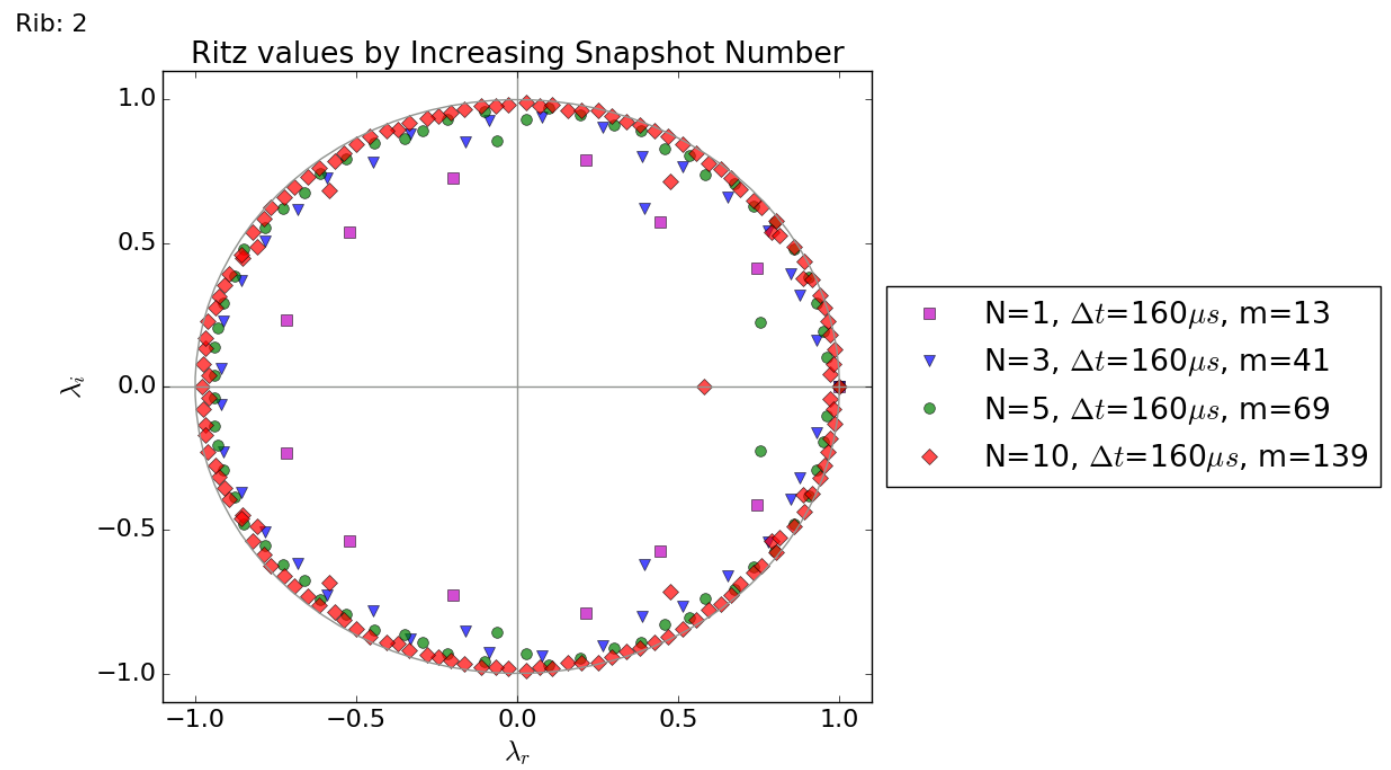

Figure A.5: Ritz values for $\Delta t=160 \mu s$. 


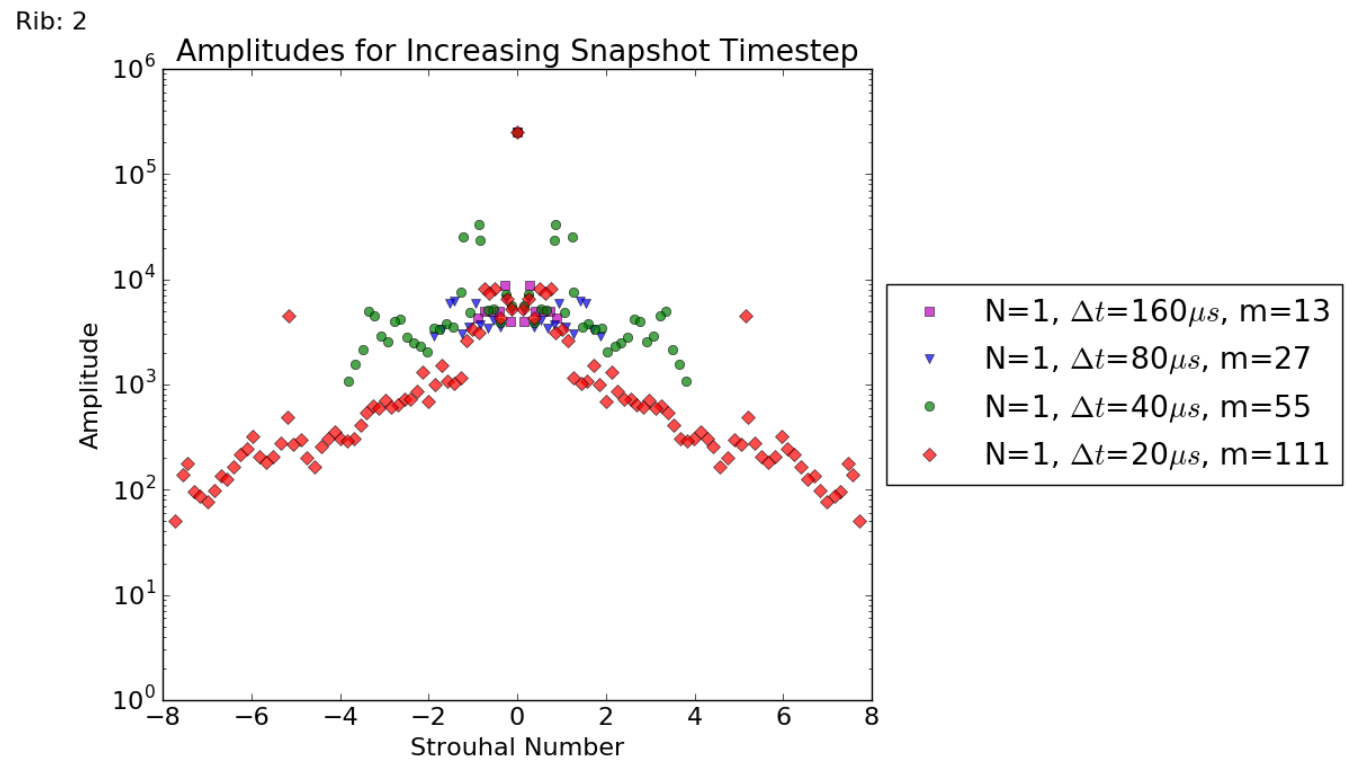

Figure A.6: DMD amplitude convergence, $N=1$.

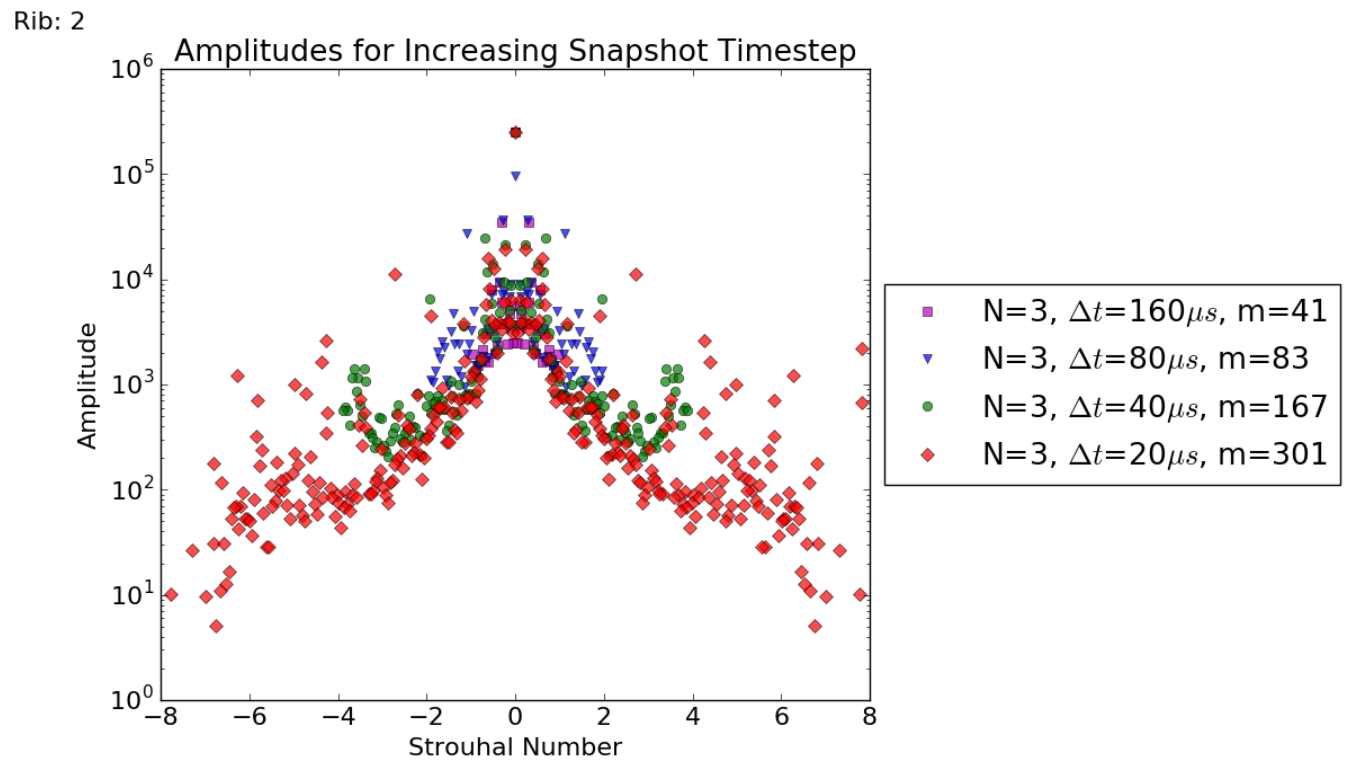

Figure A.7: DMD amplitude convergence, $N=3$. 


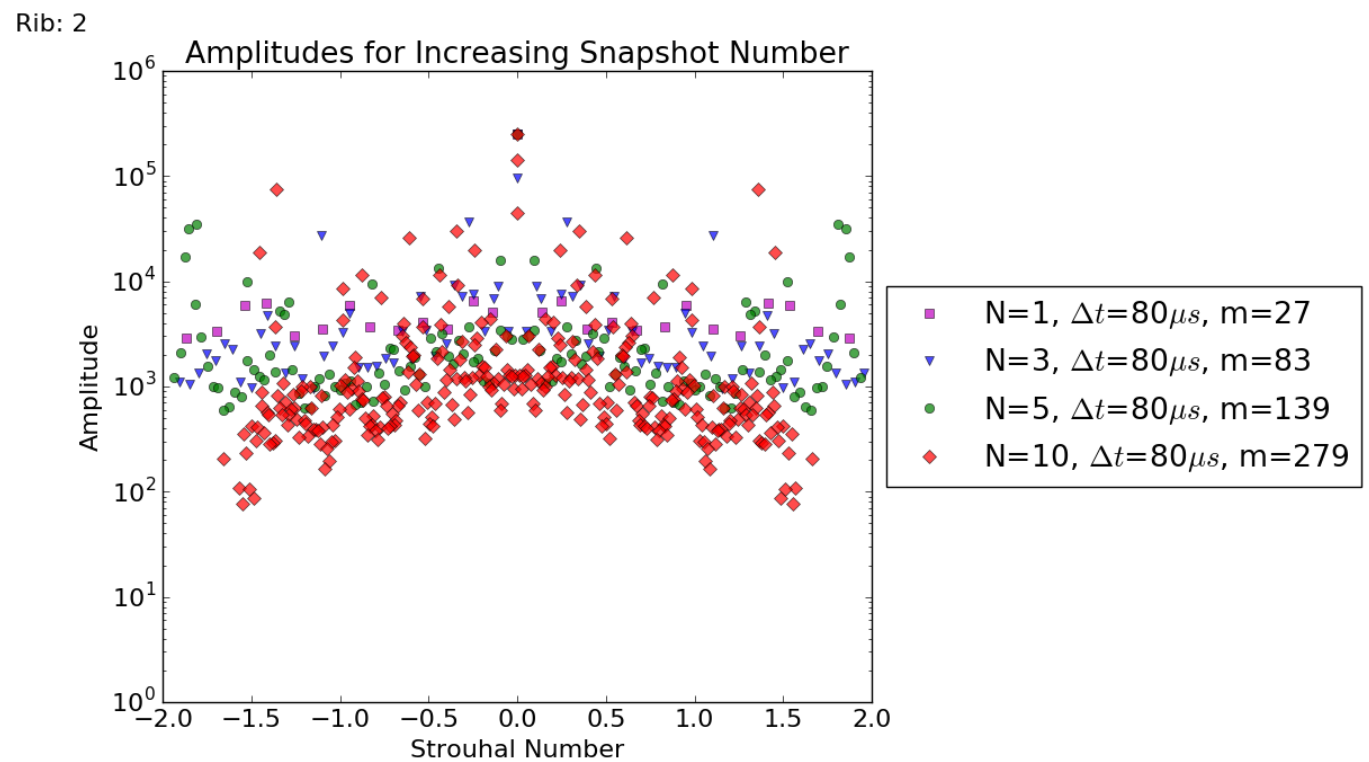

Figure A.8: DMD amplitude convergence, $\Delta t=80 \mu s$.

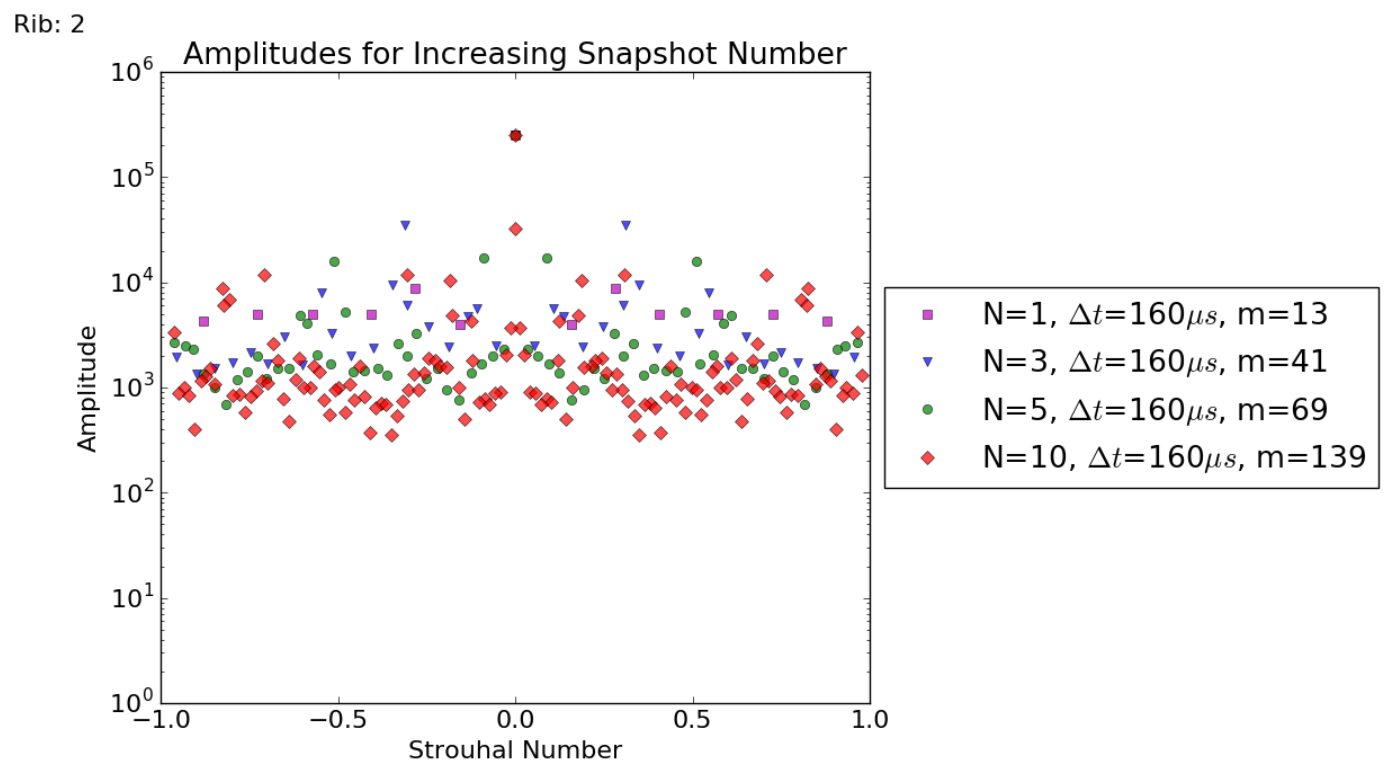

Figure A.9: DMD amplitude convergence, $\Delta t=160 \mu \mathrm{s}$. 


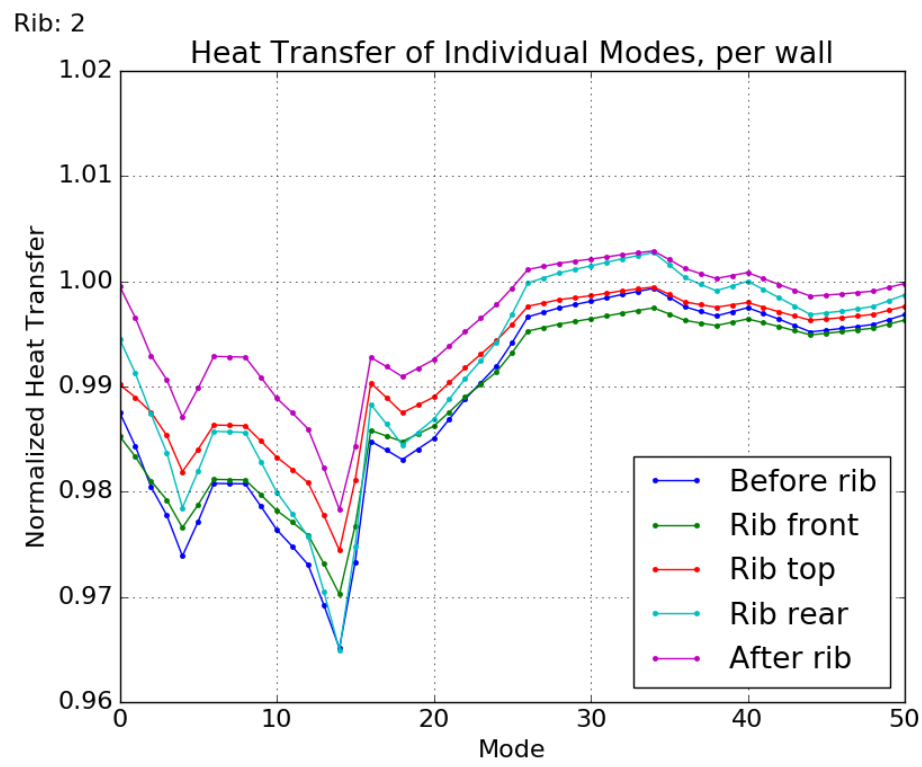

Figure A.10: Heat Transfer for individual DMD modes, split by walls as defined in figure 4.16. Analagous to figure 4.14 


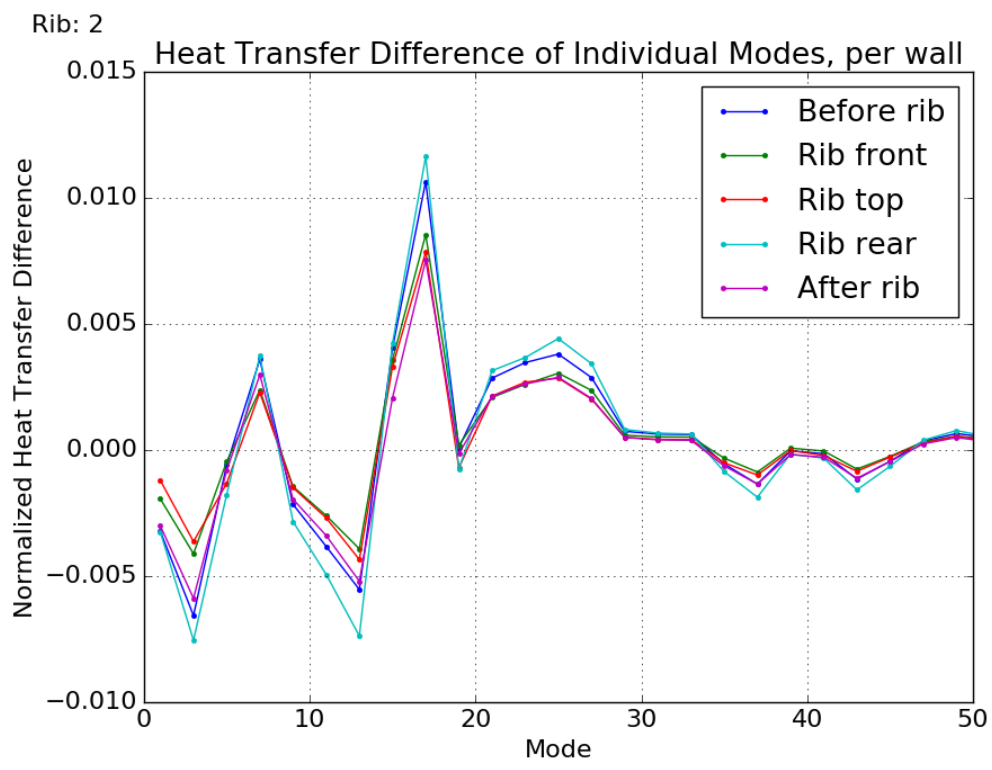

Figure A.11: Heat Transfer for individual DMD modes, split by walls as defined in figure 4.16. Analgous to figure 4.15

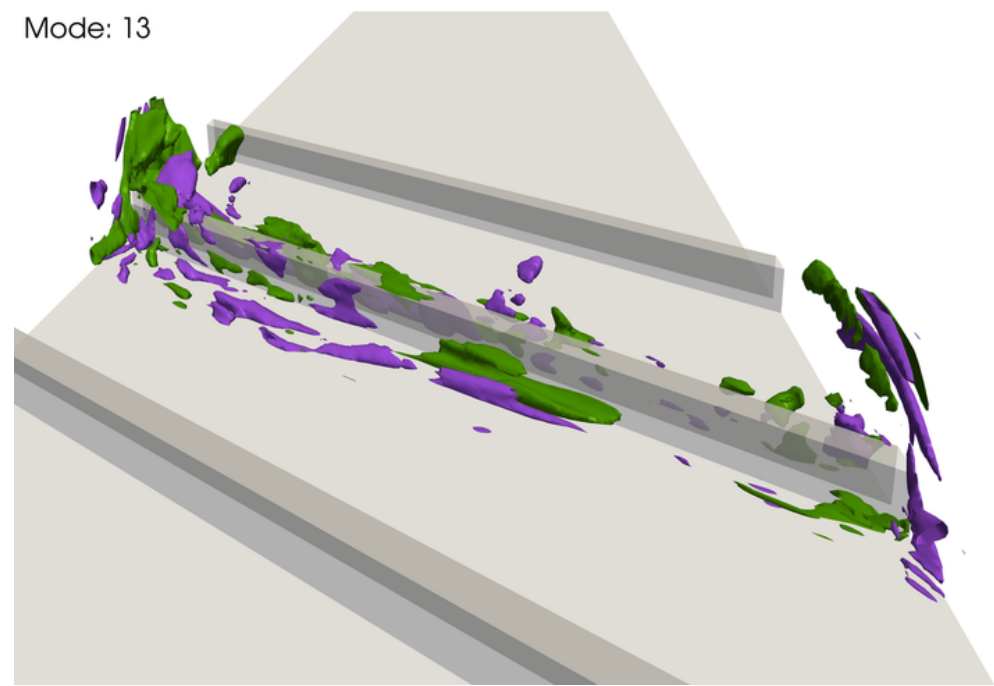

Figure A.12: 3D mode shape, Mode 13, front view. Used for case D1 


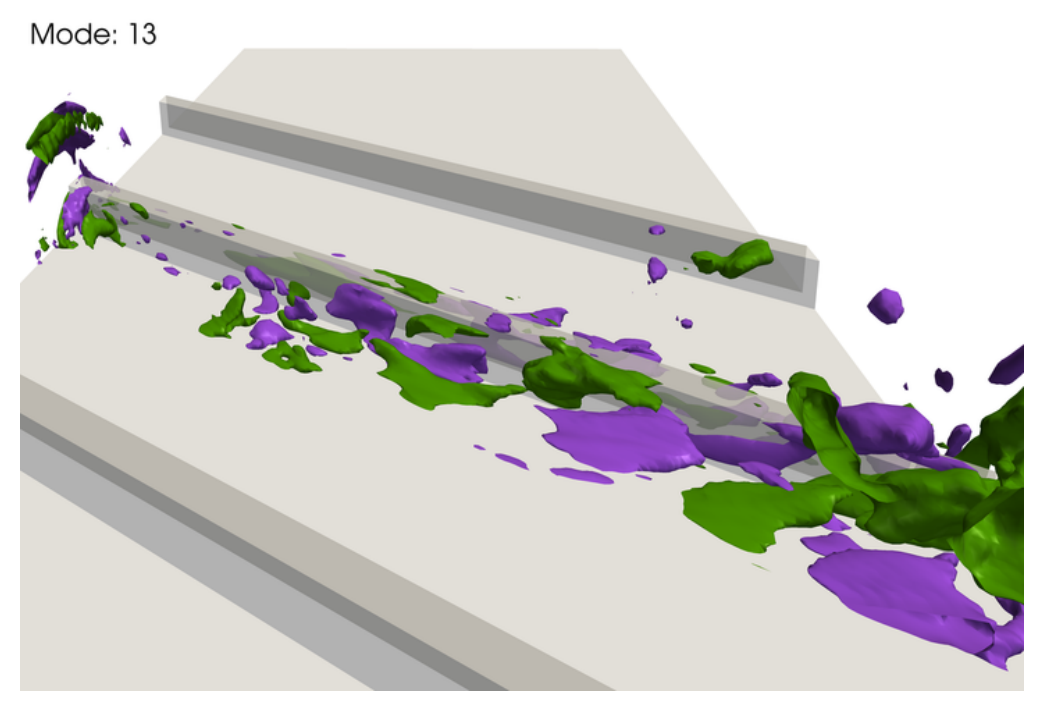

Figure A.13: 3D mode shape, Mode 13, rear view. Used for case D1

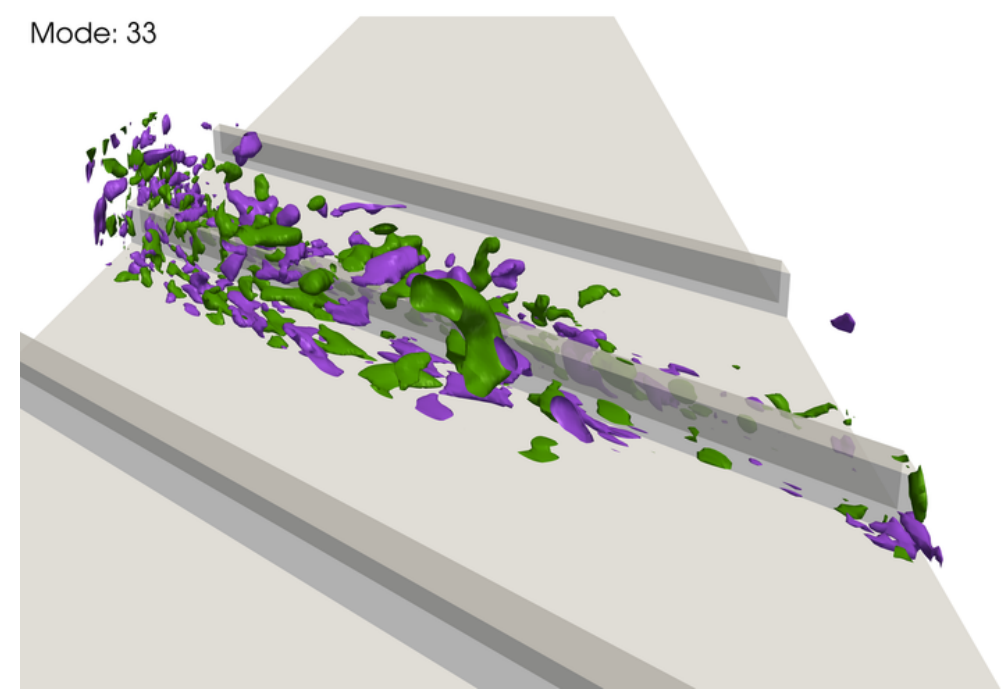

Figure A.14: 3D mode shape, Mode 33, front view. Used for case D2 


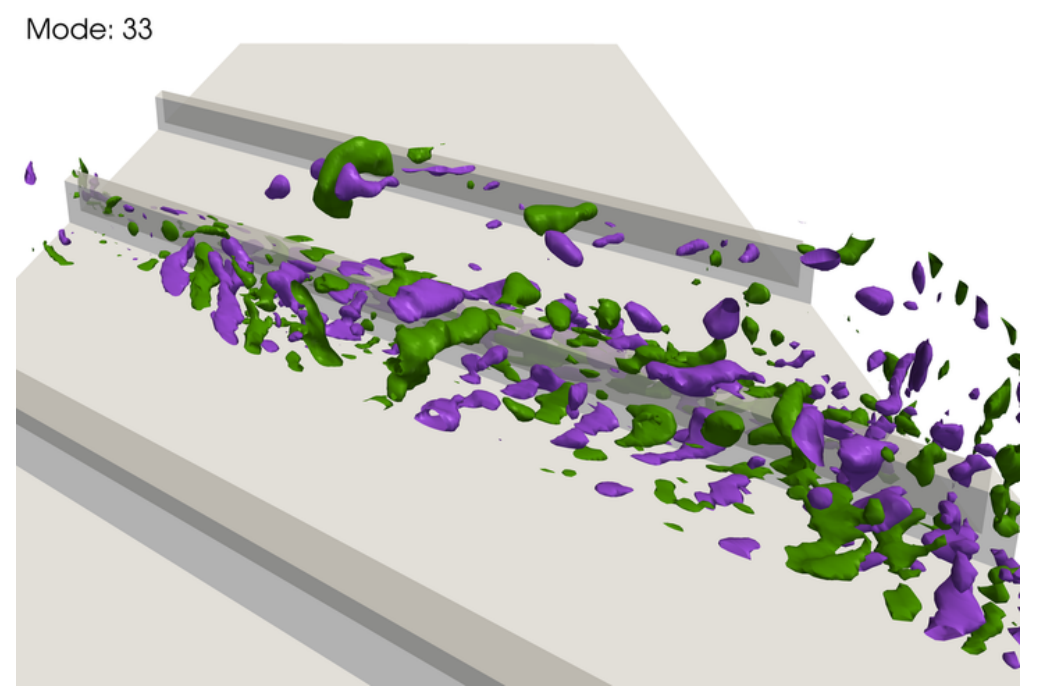

Figure A.15: 3D mode shape, Mode 33, rear view. Used for case D2

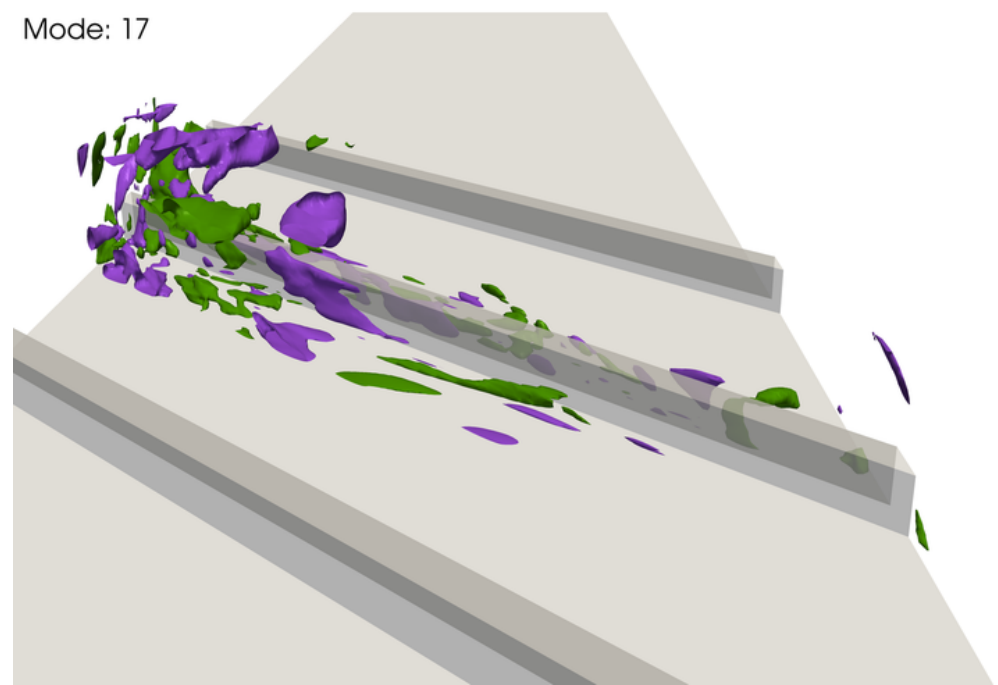

Figure A.16: 3D mode shape, Mode 17, front view. Used for case D3 


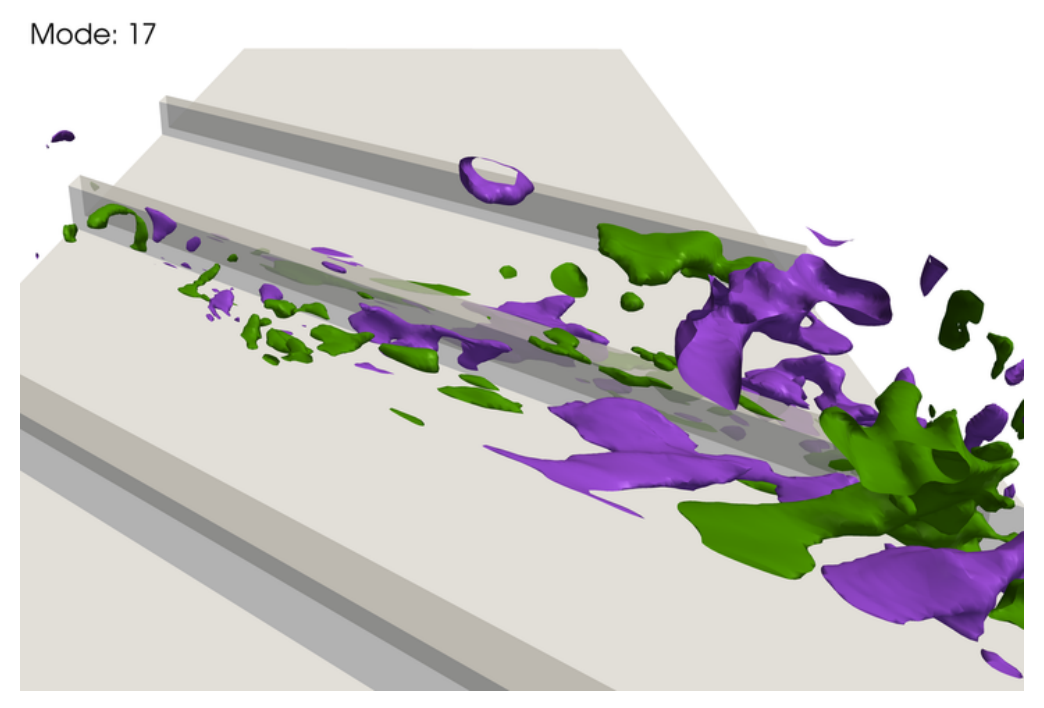

Figure A.17: 3D mode shape, Mode 17, rear view. Used for case D3

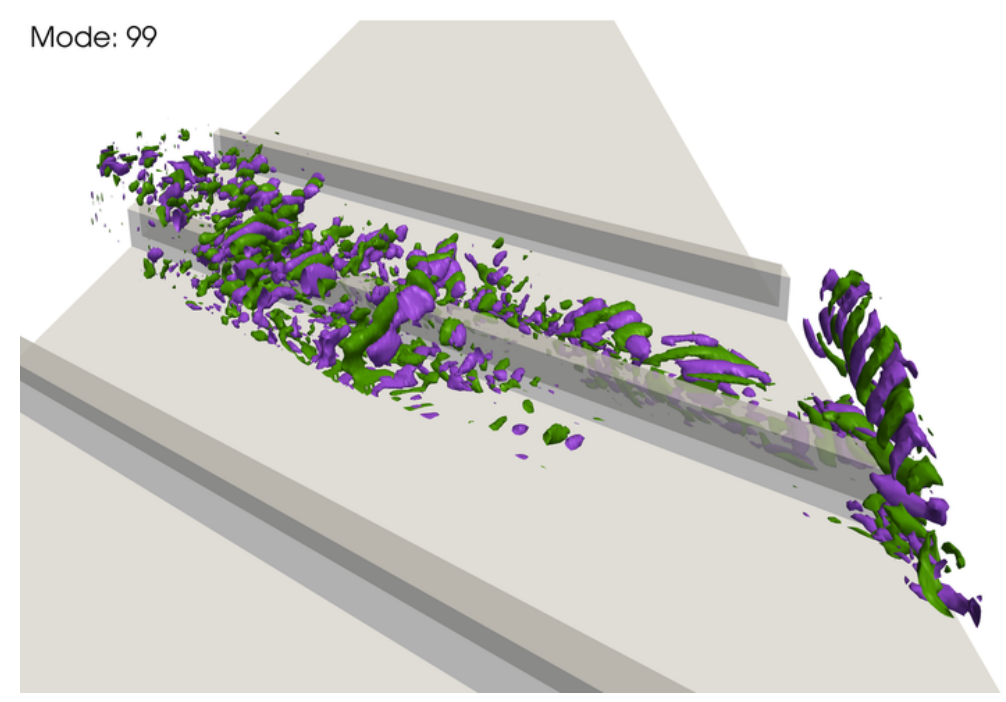

Figure A.18: 3D mode shape, Mode 99, front view. Used for case D4 


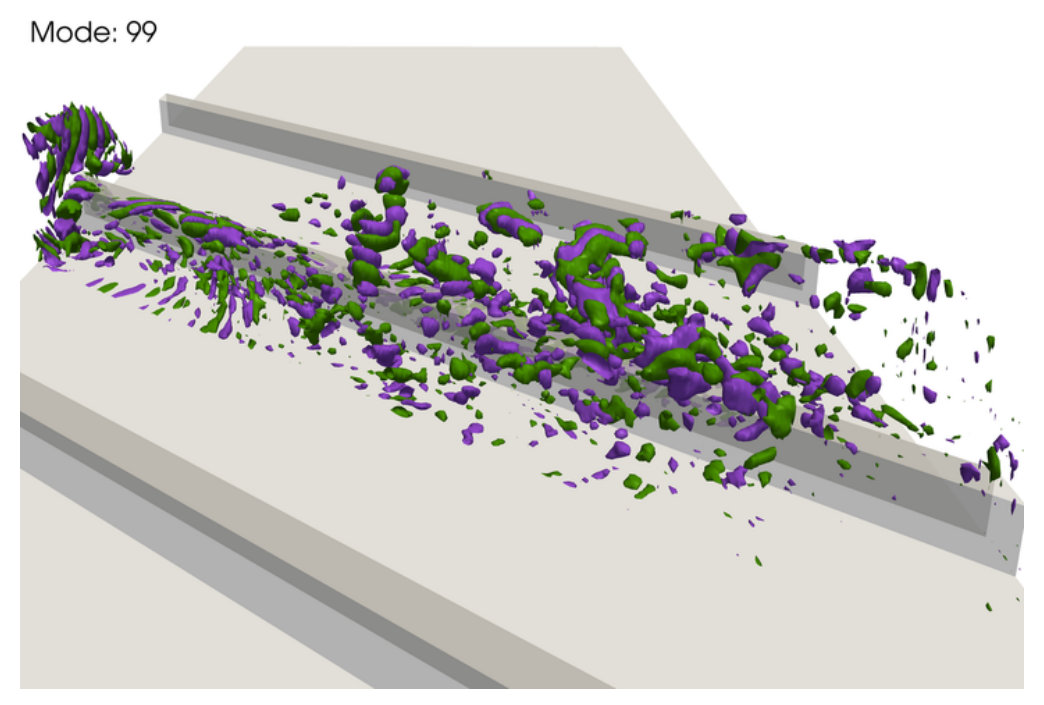

Figure A.19: 3D mode shape, Mode 99, rear view. Used for case D4

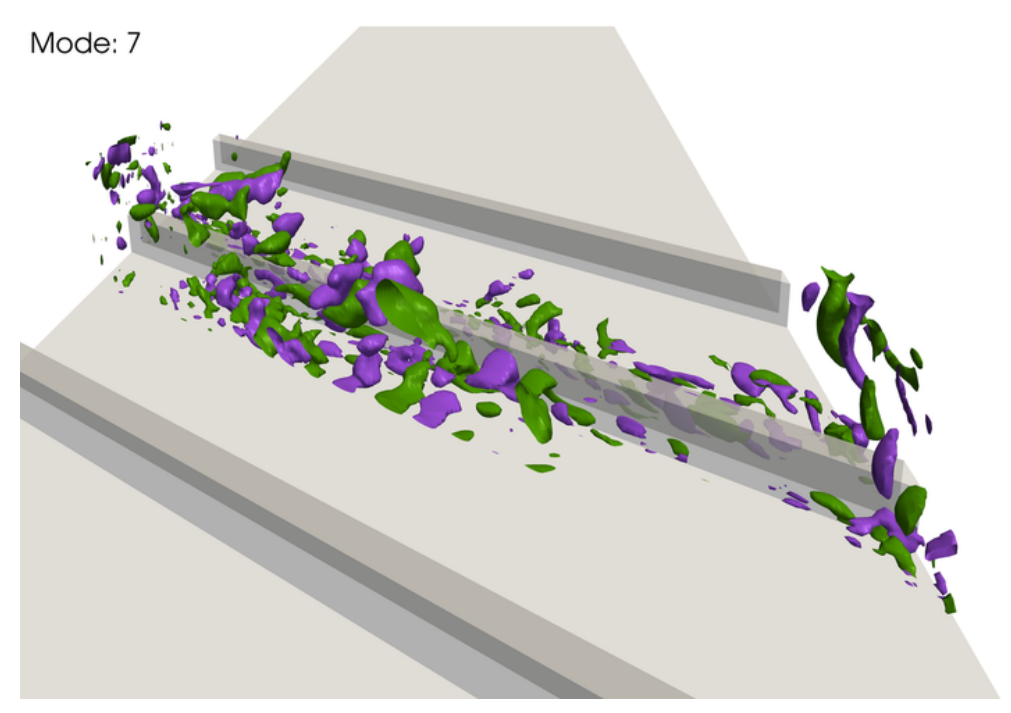

Figure A.20: 3D mode shape, Mode 7, front view. Used for case D5 


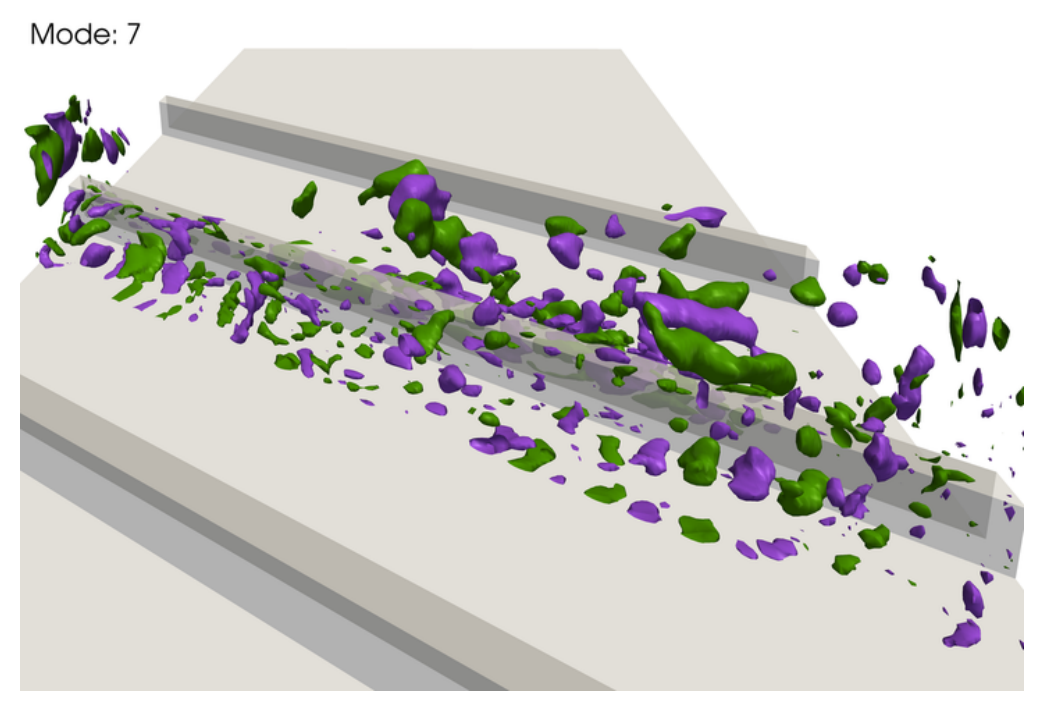

Figure A.21: 3D mode shape, Mode 7, rear view. Used for case D5

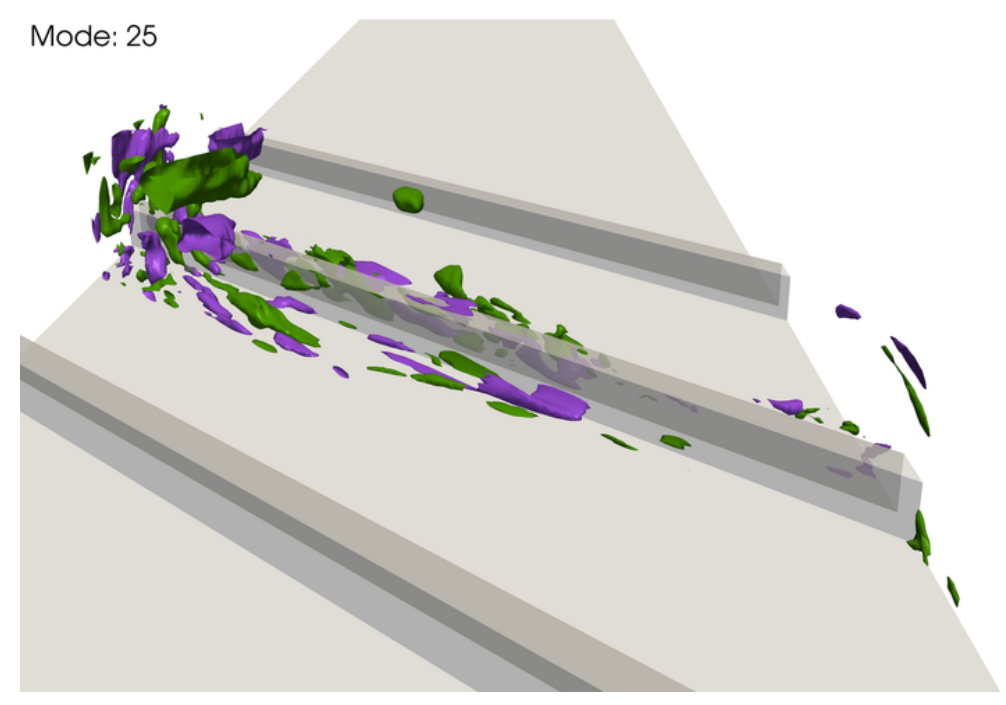

Figure A.22: 3D mode shape, Mode 25, front view. Used for case D6 


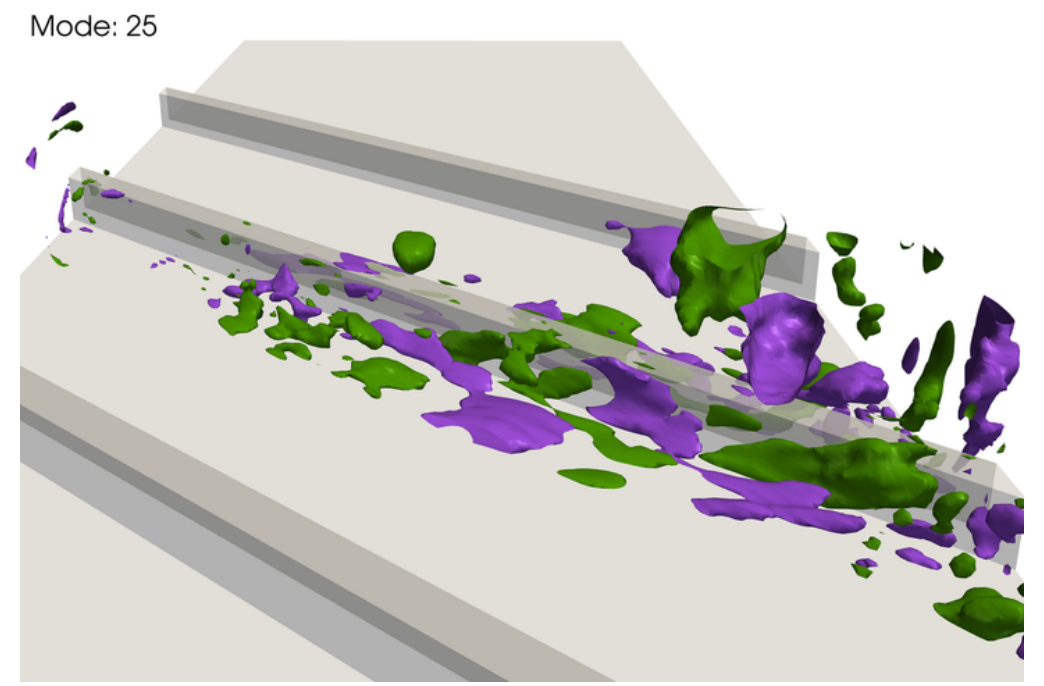

Figure A.23: 3D mode shape, Mode 25, rear view. Used for case D6 
APPENDIX B: MORPHED CASE POST-PROCESSING 


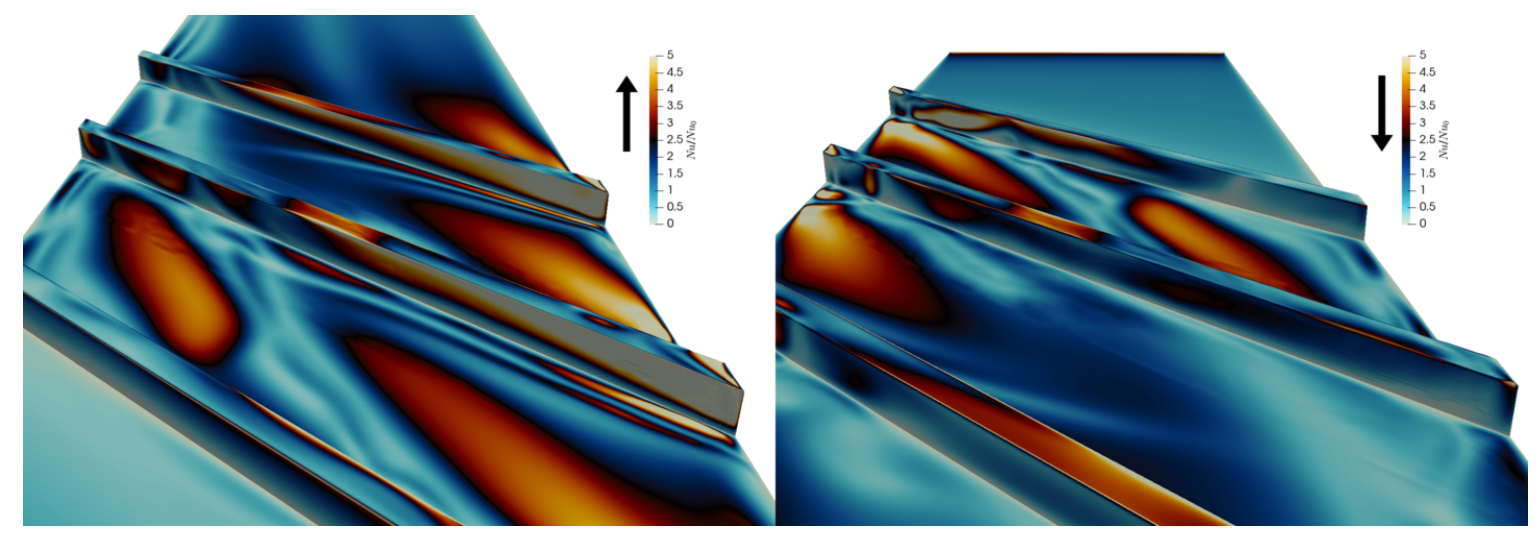

Figure B.1: Nusselt ratio, case D1. As viewed from the front (left) and rear (right). Flow moves with the arrow.

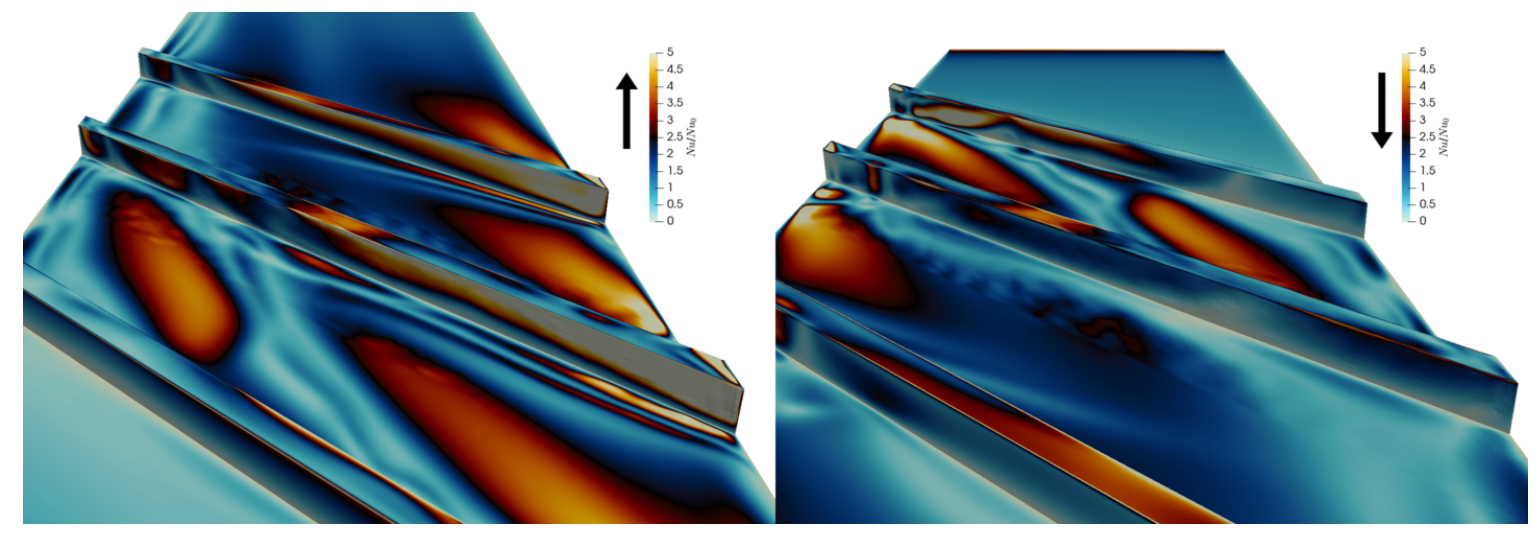

Figure B.2: Nusselt ratio, case D2. As viewed from the front (left) and rear (right). Flow moves with the arrow. 


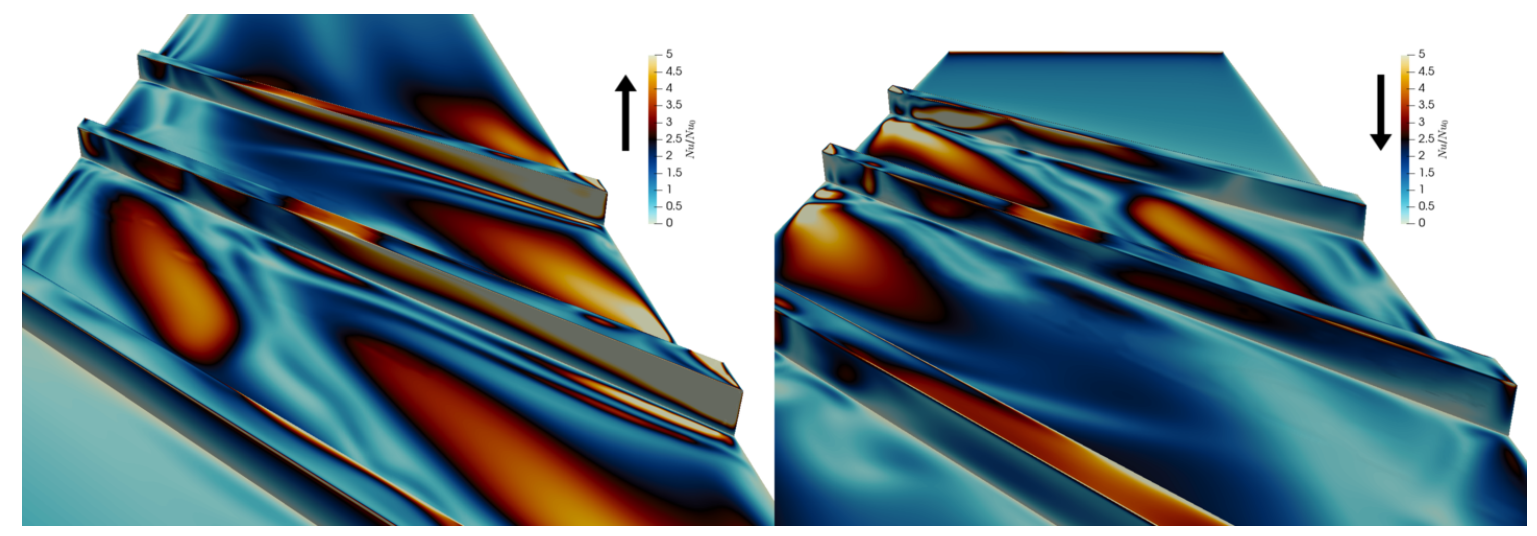

Figure B.3: Nusselt ratio, case D3. As viewed from the front (left) and rear (right). Flow moves with the arrow.

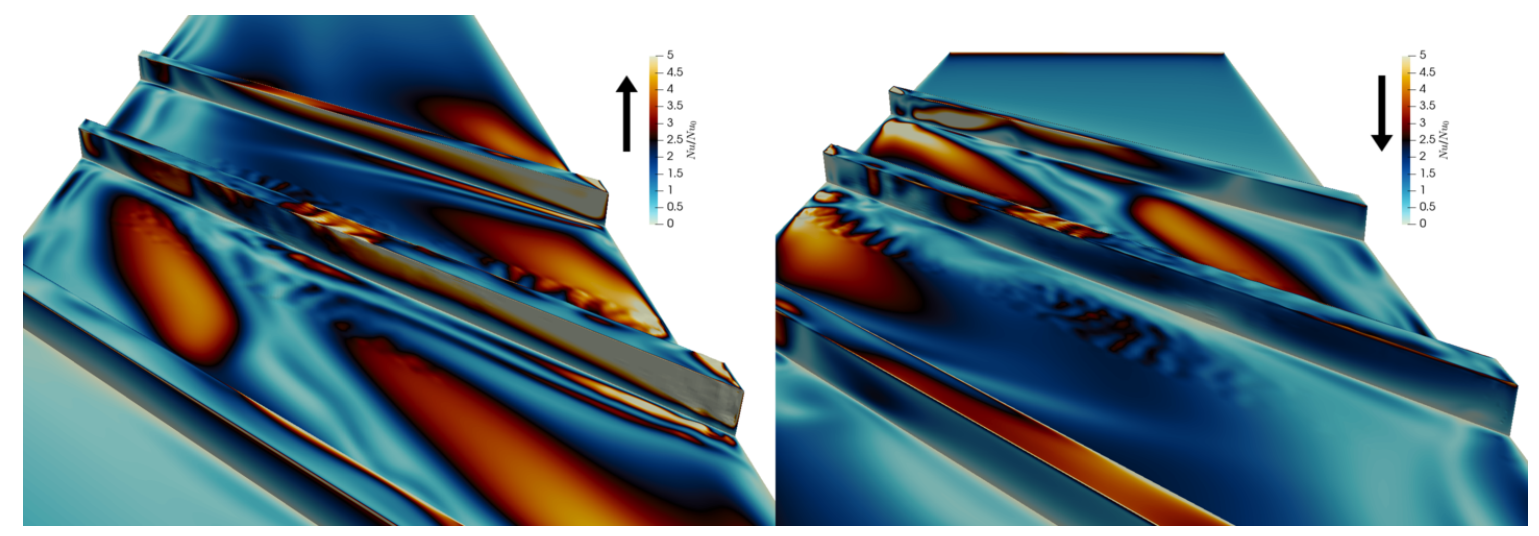

Figure B.4: Nusselt ratio, case D4. As viewed from the front (left) and rear (right). Flow moves with the arrow. 


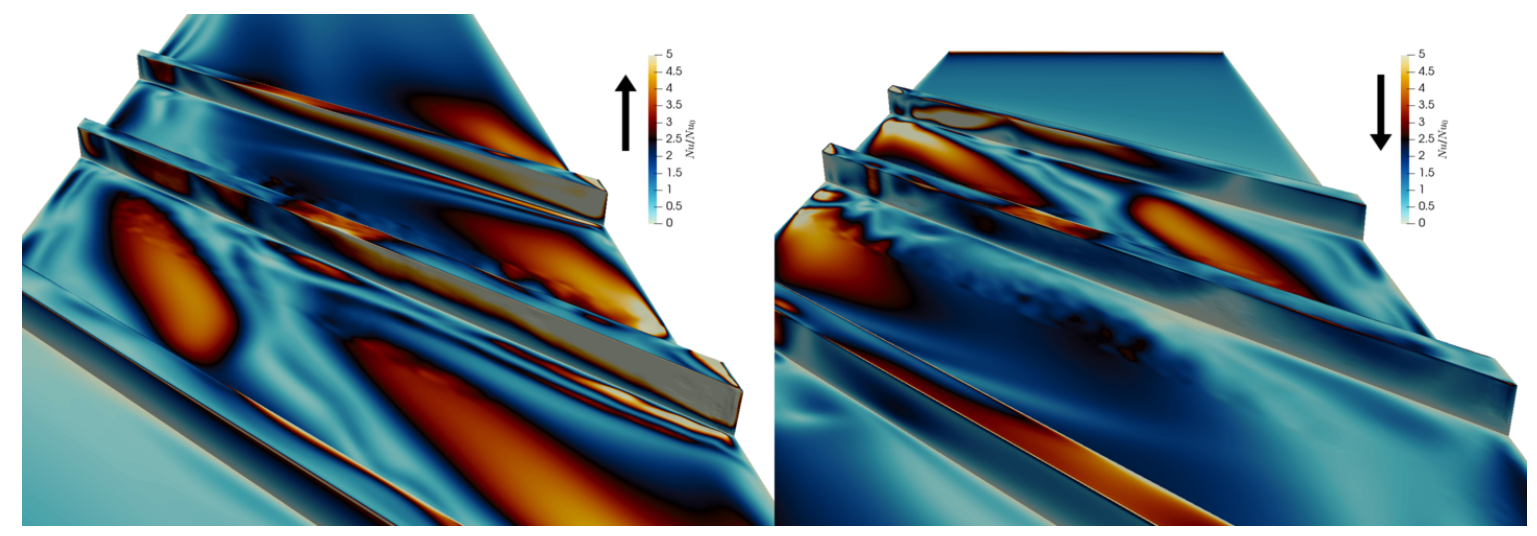

Figure B.5: Nusselt ratio, case D5. As viewed from the front (left) and rear (right). Flow moves with the arrow.

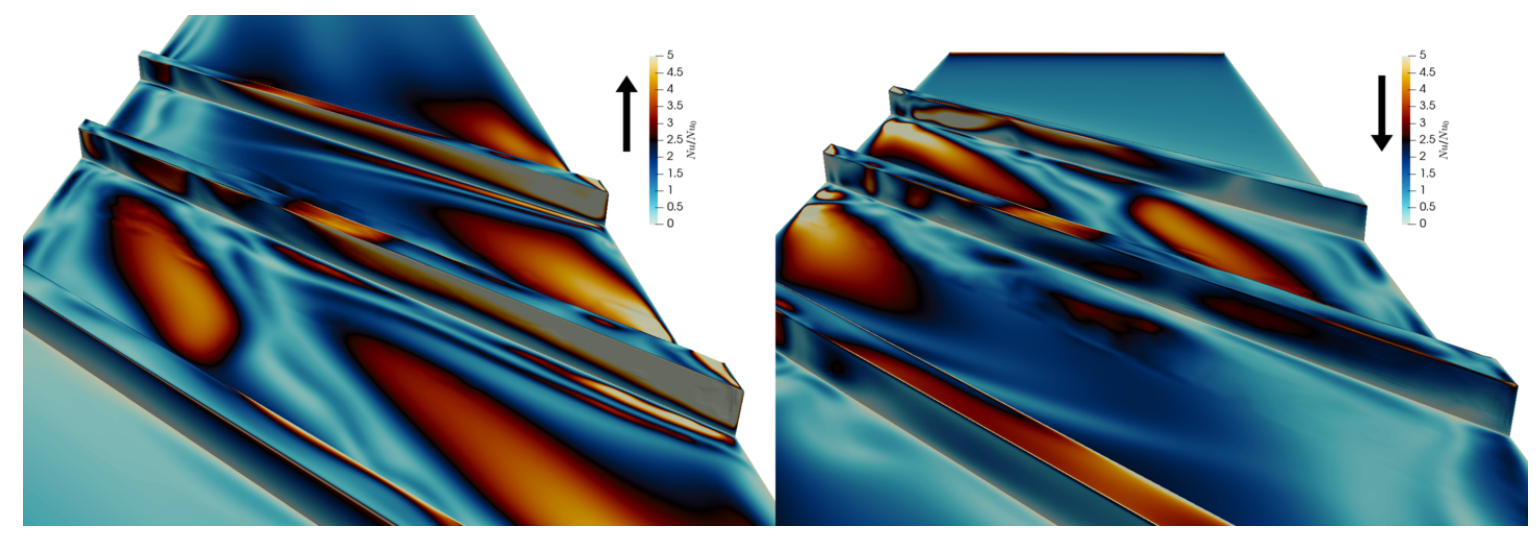

Figure B.6: Nusselt ratio, case D6. As viewed from the front (left) and rear (right). Flow moves with the arrow. 


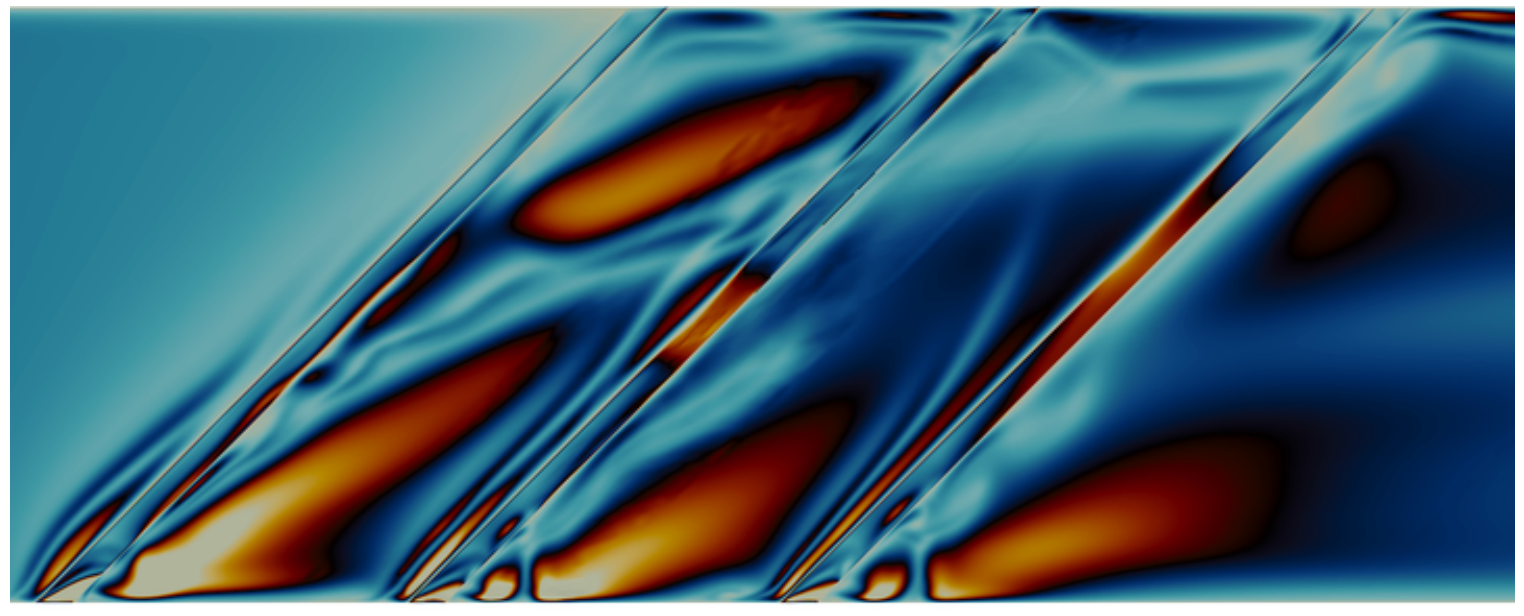

$\mathrm{Nu} / \mathrm{Nu} u_{0}$

$\begin{array}{lllllllllll}0 & 0.5 & 1 & 1.5 & 2 & 2.5 & 3 & 3.5 & 4 & 4.5 & 5\end{array}$

Figure B.7: Nusselt ratio, case D1. As viewed from the top. Flow moves from left to right.

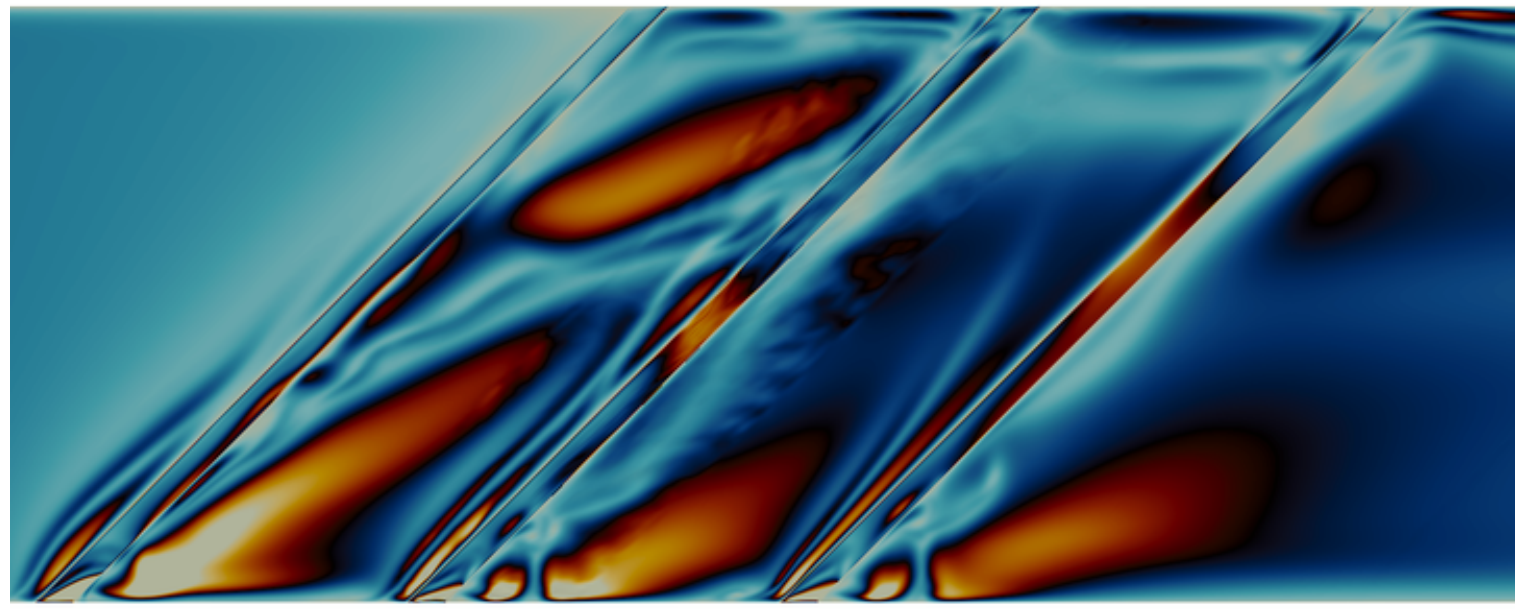

$\mathrm{Nu} / \mathrm{Nu} u_{0}$

$\begin{array}{lllllllllll}0 & 0.5 & 1 & 1.5 & 2 & 2.5 & 3 & 3.5 & 4 & 4.5 & 5\end{array}$

Figure B.8: Nusselt ratio, case D2. As viewed from the top. Flow moves from left to right. 


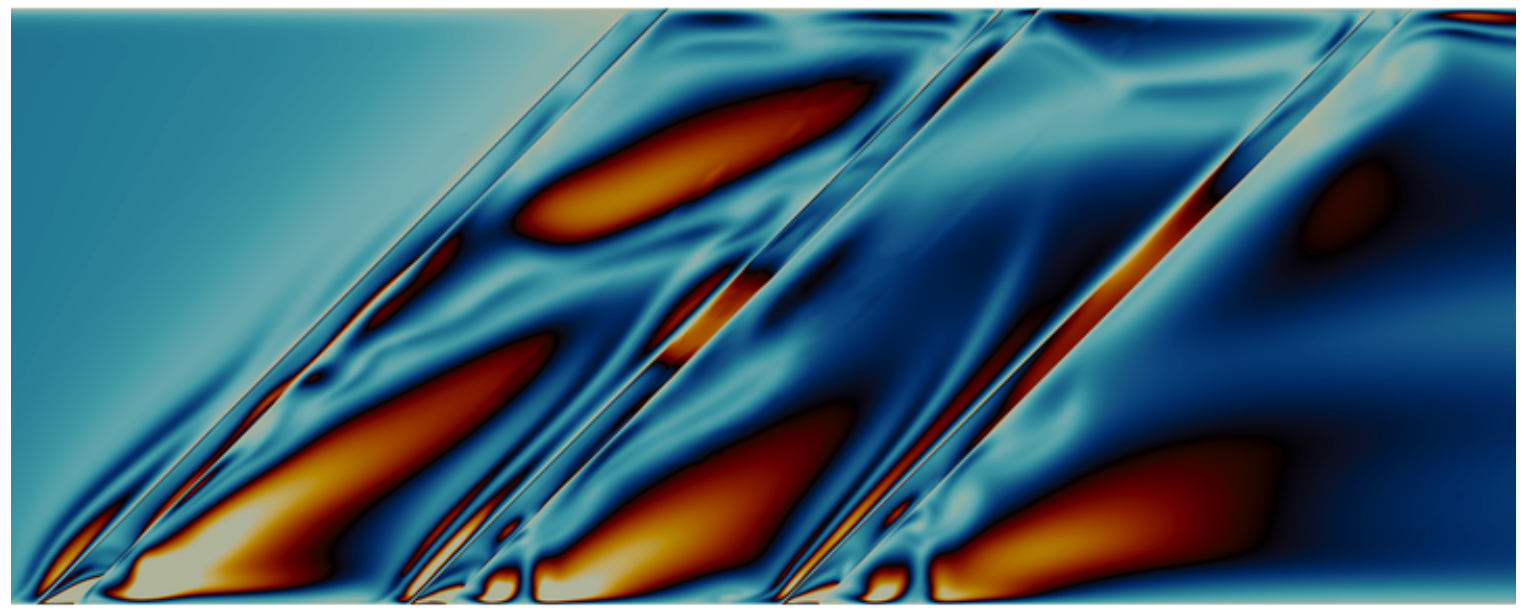

$\mathrm{Nu} / \mathrm{Nu}_{0}$

$\begin{array}{lllllllllll}0 & 0.5 & 1 & 1.5 & 2 & 2.5 & 3 & 3.5 & 4 & 4.5 & 5\end{array}$

Figure B.9: Nusselt ratio, case D3. As viewed from the top. Flow moves from left to right.

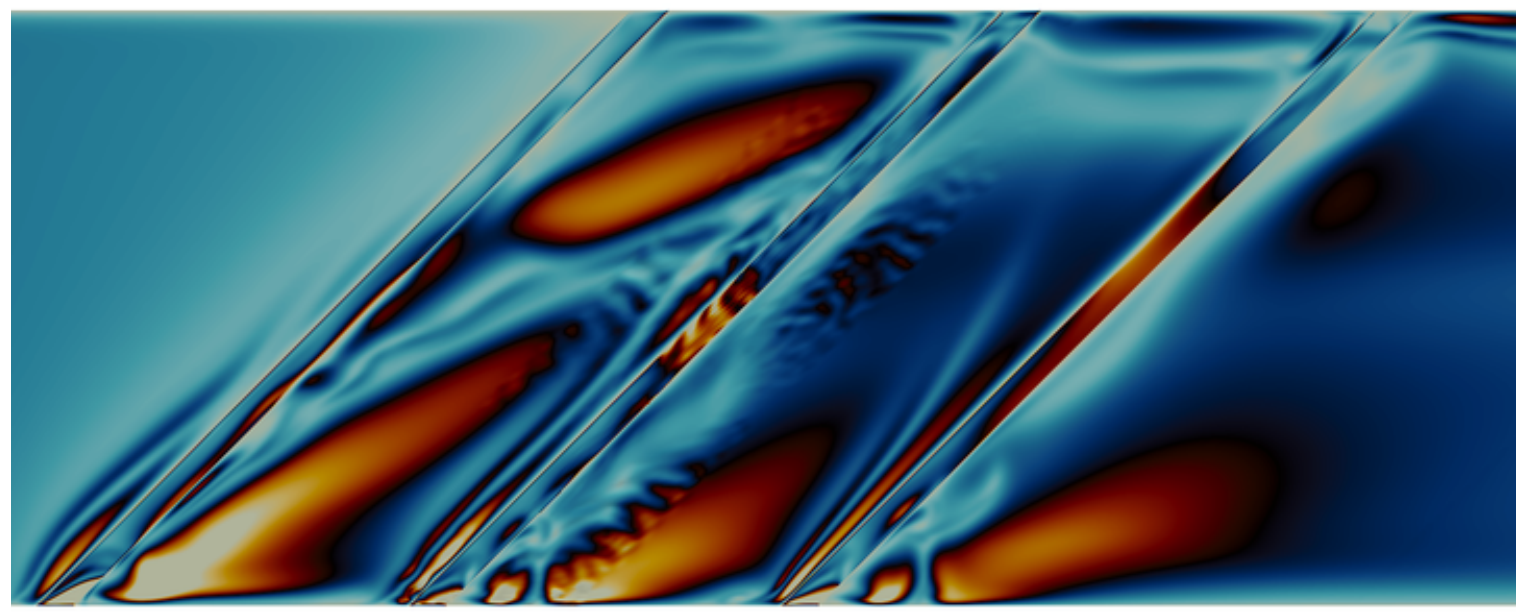

$\mathrm{Nu} / \mathrm{Nu} u_{0}$

$\begin{array}{lllllllllll}0 & 0.5 & 1 & 1.5 & 2 & 2.5 & 3 & 3.5 & 4 & 4.5 & 5\end{array}$

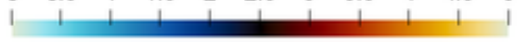

Figure B.10: Nusselt ratio, case D4. As viewed from the top. Flow moves from left to right. 


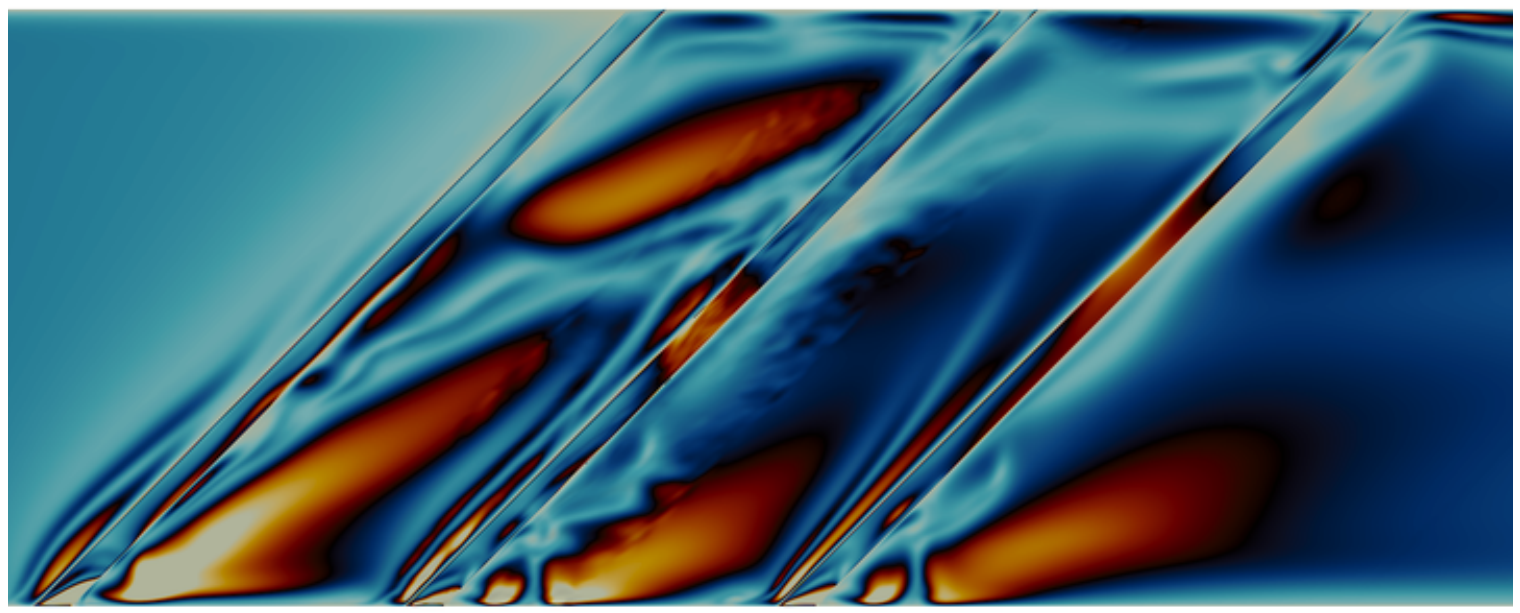

$\mathrm{Nu} / \mathrm{Nu} u_{0}$

$\begin{array}{lllllllllll}0 & 0.5 & 1 & 1.5 & 2 & 2.5 & 3 & 3.5 & 4 & 4.5 & 5\end{array}$

Figure B.11: Nusselt ratio, case D5. As viewed from the top. Flow moves from left to right.

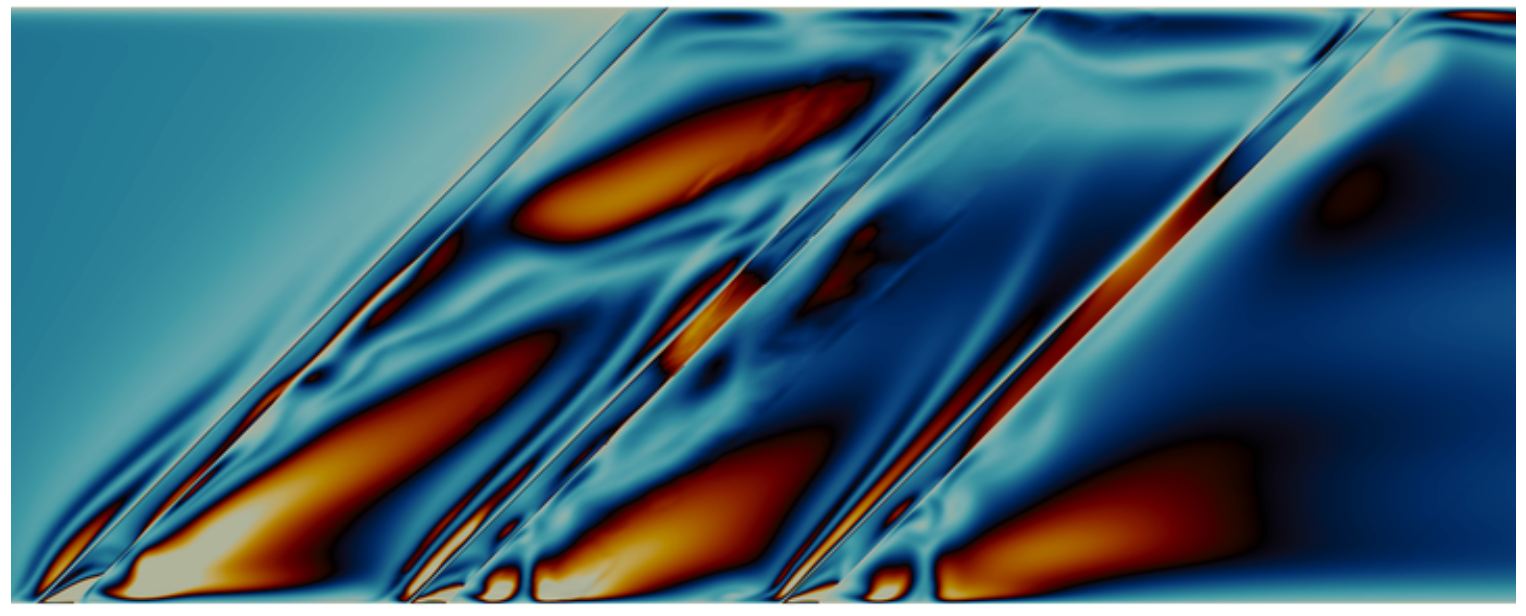

$\mathrm{Nu} / \mathrm{Nu} u_{0}$

$\begin{array}{lllllllllll}0 & 0.5 & 1 & 1.5 & 2 & 2.5 & 3 & 3.5 & 4 & 4.5 & 5\end{array}$

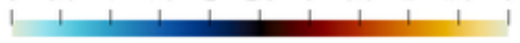

Figure B.12: Nusselt ratio, case D6. As viewed from the top. Flow moves from left to right. 


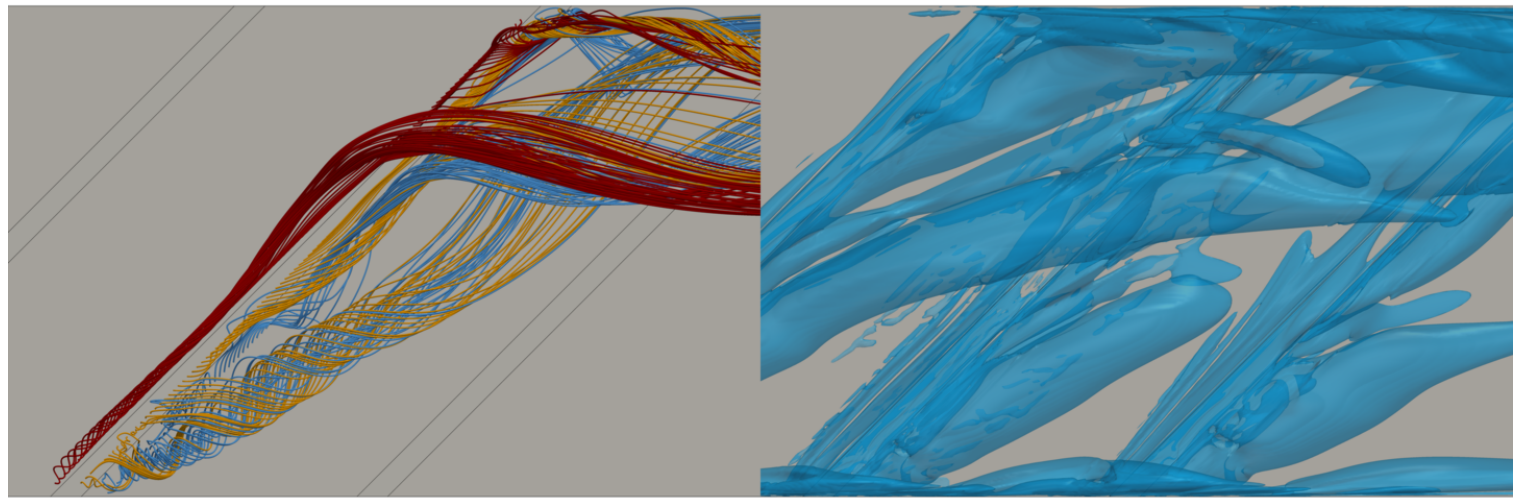

Figure B.13: Streamlines (left) and Q-criterion (right) of case D1. Red streamlines are seeded from the front of the rib, gold from the top, and blue from the rear. The blue isosurface is of rotational Q-criterion.

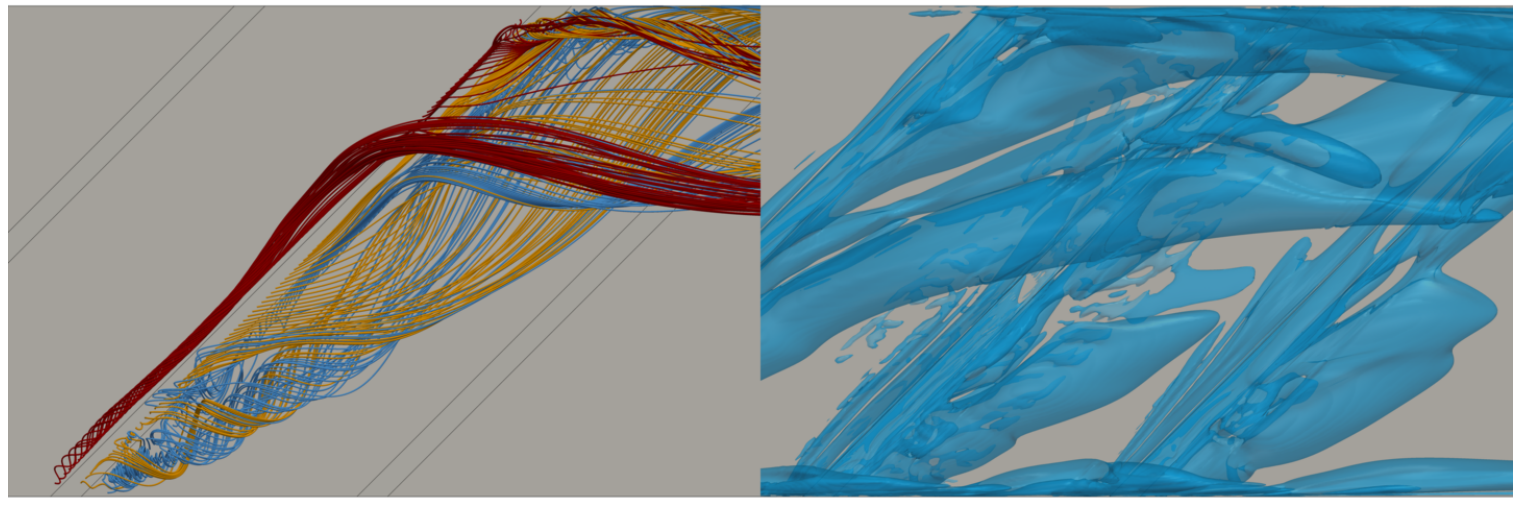

Figure B.14: Streamlines (left) and Q-criterion (right) of case D2. Red streamlines are seeded from the front of the rib, gold from the top, and blue from the rear. The blue isosurface is of rotational Q-criterion. 


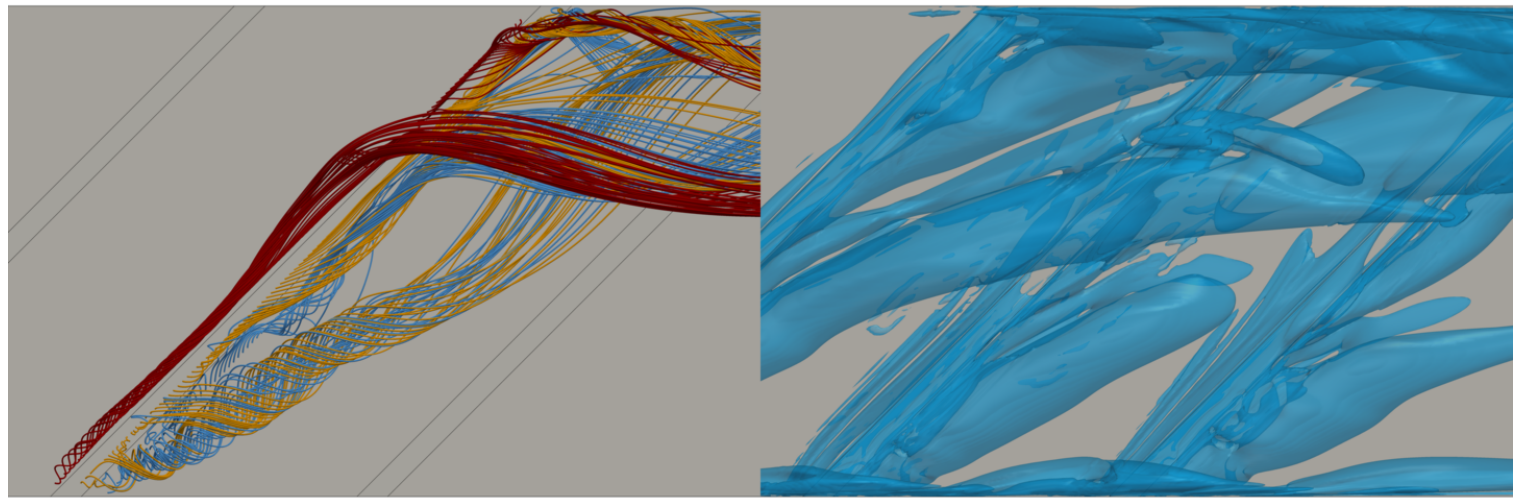

Figure B.15: Streamlines (left) and Q-criterion (right) of case D3. Red streamlines are seeded from the front of the rib, gold from the top, and blue from the rear. The blue isosurface is of rotational Q-criterion.

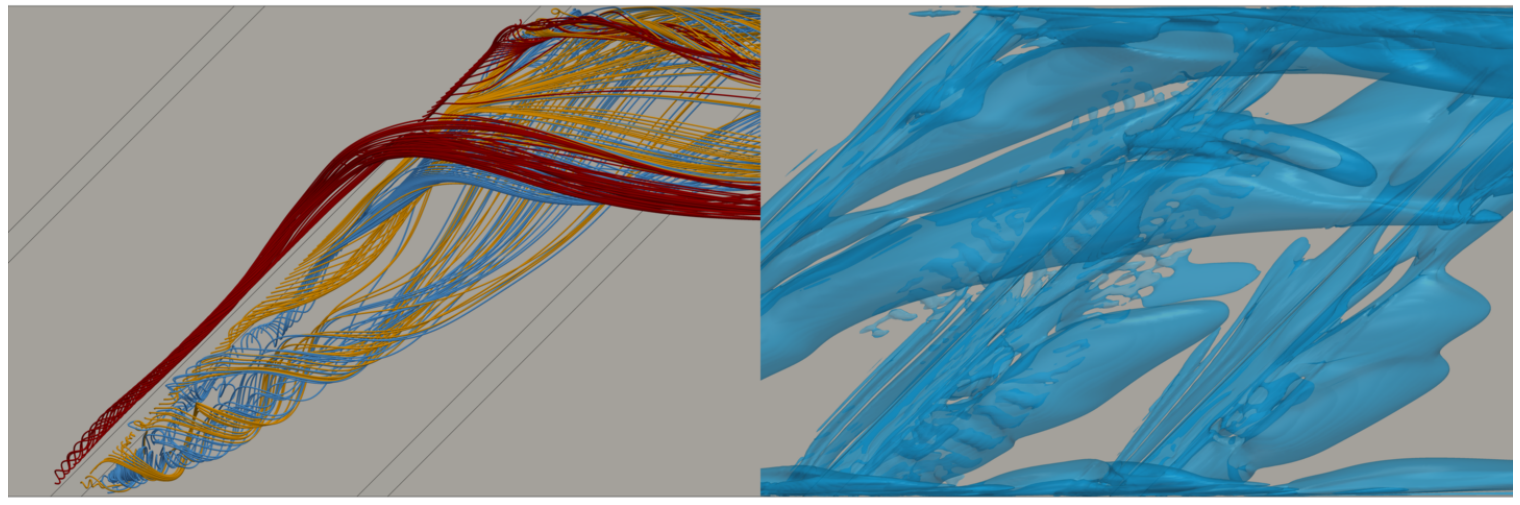

Figure B.16: Streamlines (left) and Q-criterion (right) of case D4. Red streamlines are seeded from the front of the rib, gold from the top, and blue from the rear. The blue isosurface is of rotational Q-criterion. 


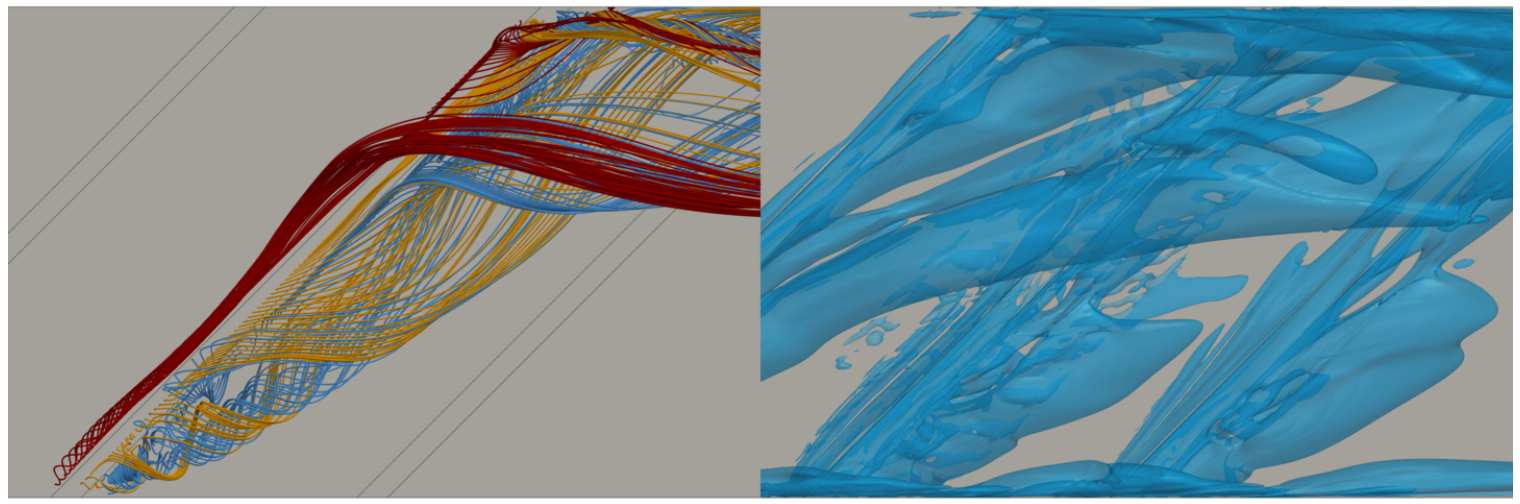

Figure B.17: Streamlines (left) and Q-criterion (right) of case D5. Red streamlines are seeded from the front of the rib, gold from the top, and blue from the rear. The blue isosurface is of rotational Q-criterion.

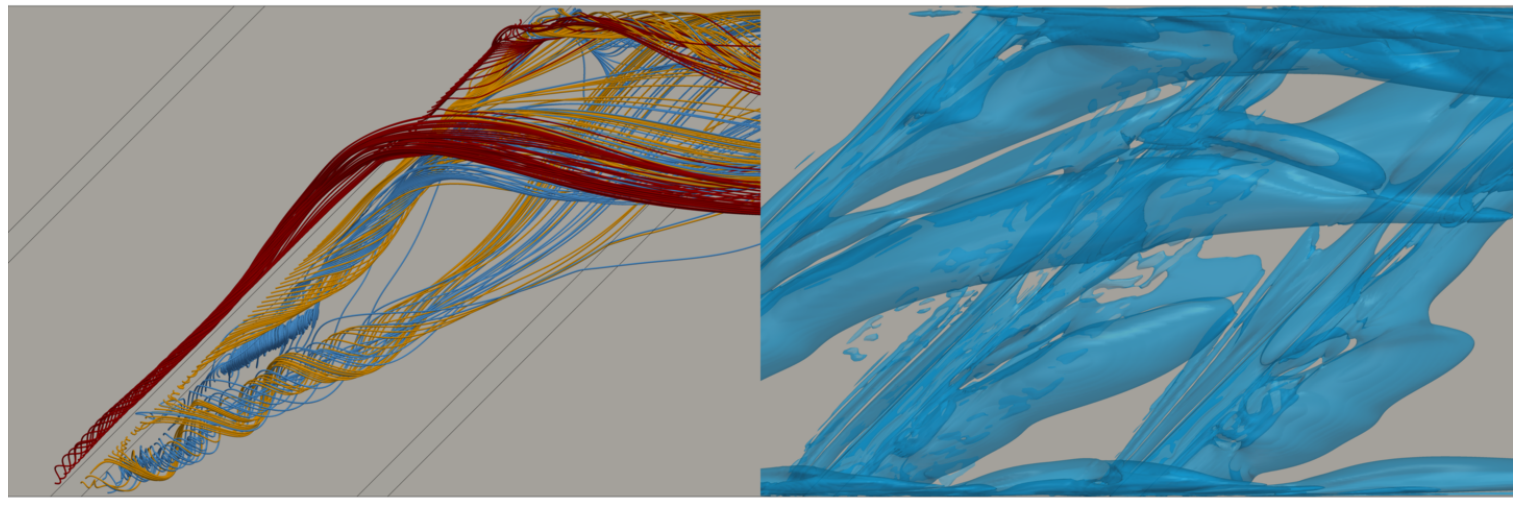

Figure B.18: Streamlines (left) and Q-criterion (right) of case D6. Red streamlines are seeded from the front of the rib, gold from the top, and blue from the rear. The blue isosurface is of rotational Q-criterion. 


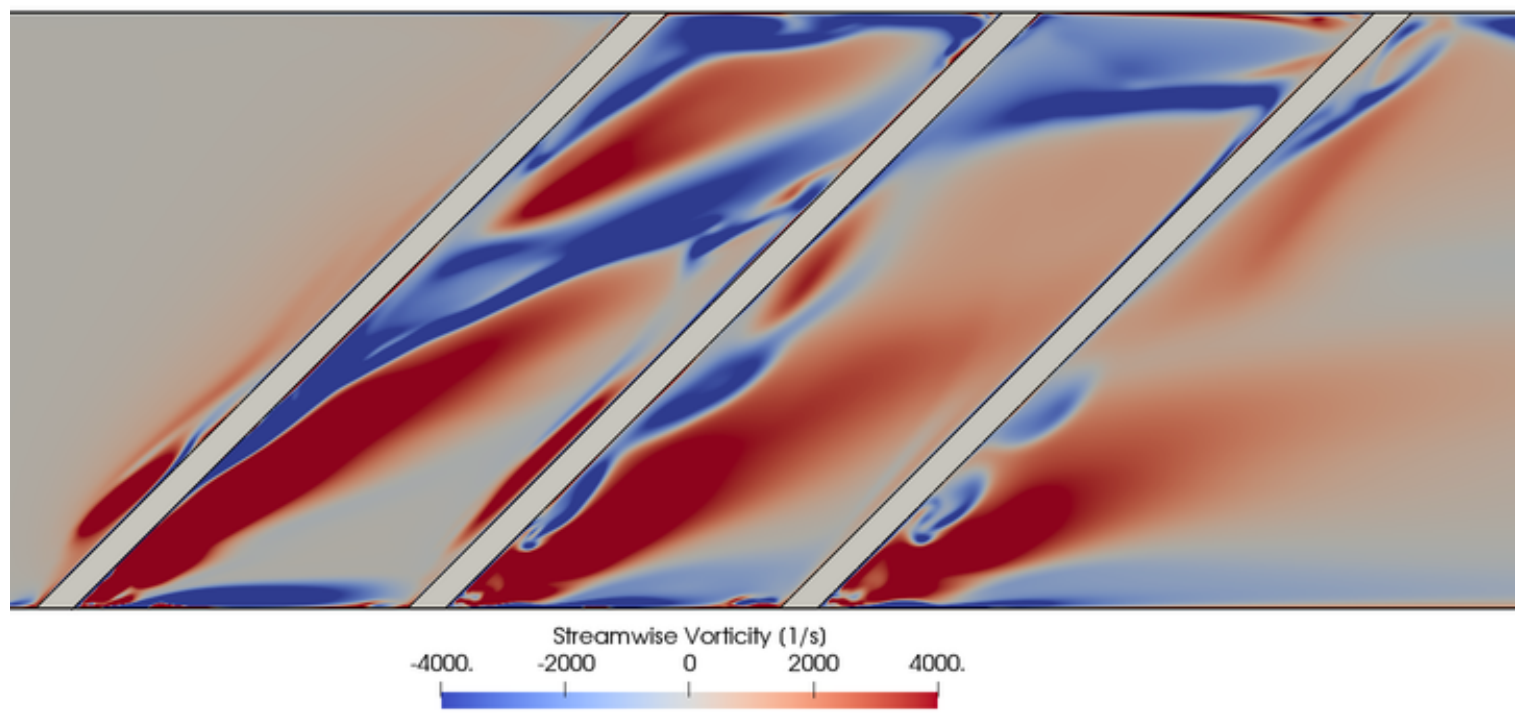

Figure B.19: Case D1 streamwise vorticity component shown on a plane section half way up the rib height. Flow moves from left to right, with positive vorticity.

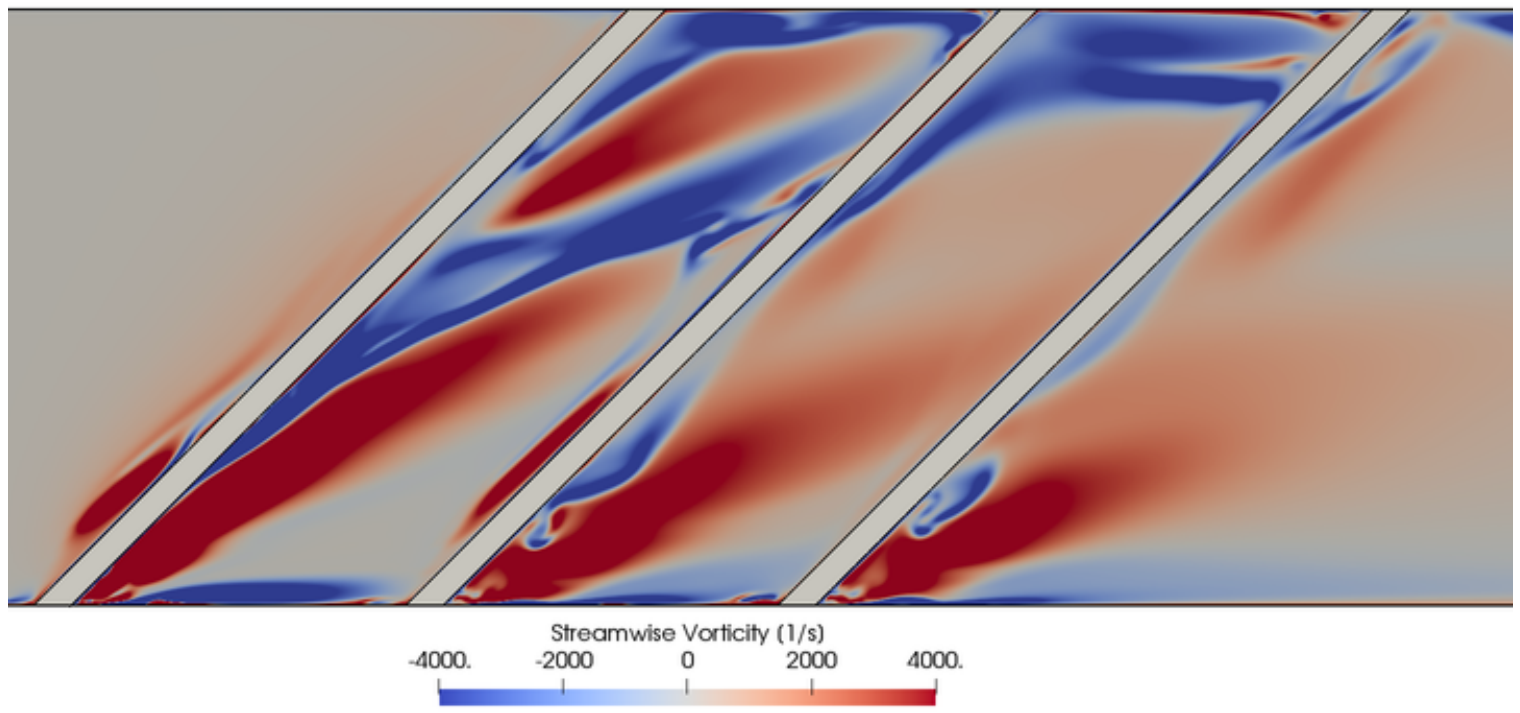

Figure B.20: Case D1 streamwise vorticity component shown on a plane section half way up the rib height. Flow moves from left to right, with positive vorticity. 


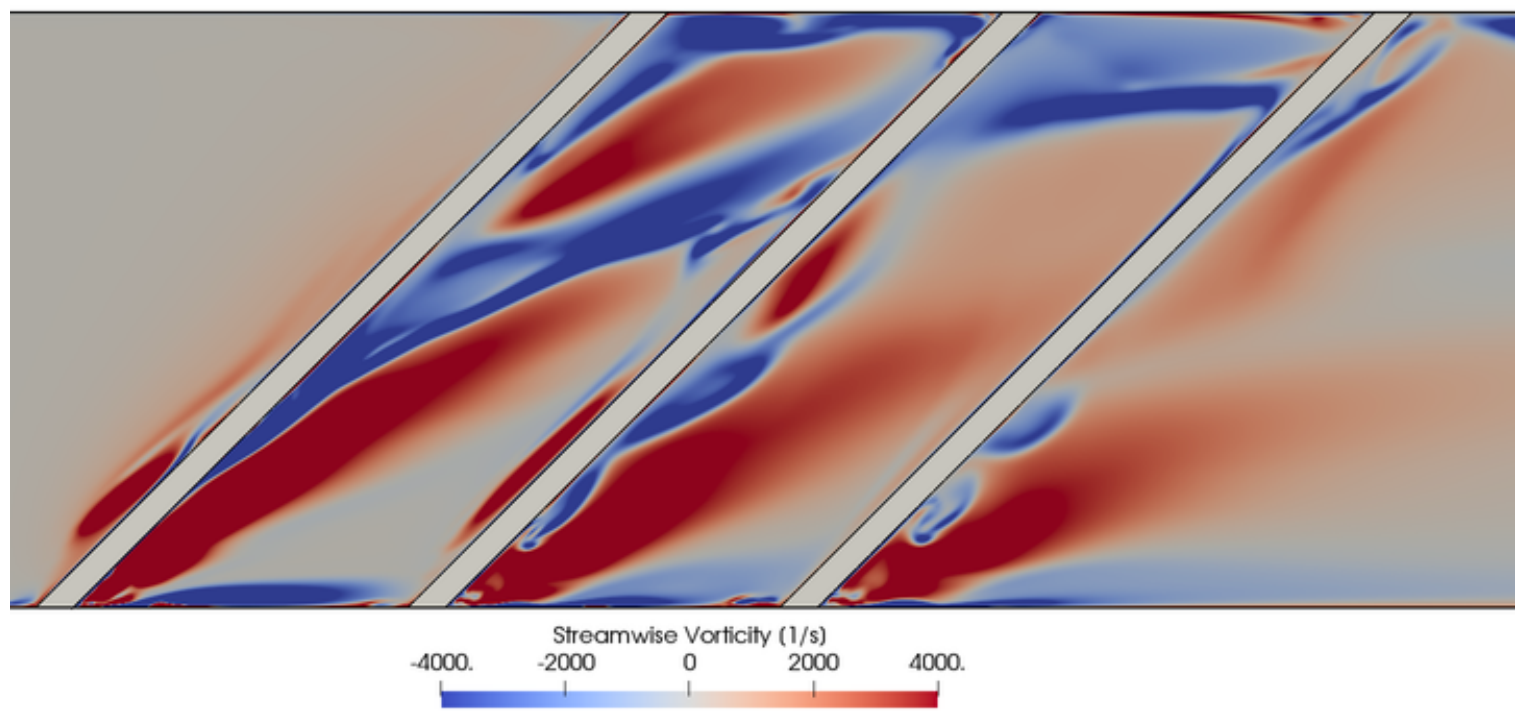

Figure B.21: Case D1 streamwise vorticity component shown on a plane section half way up the rib height. Flow moves from left to right, with positive vorticity.

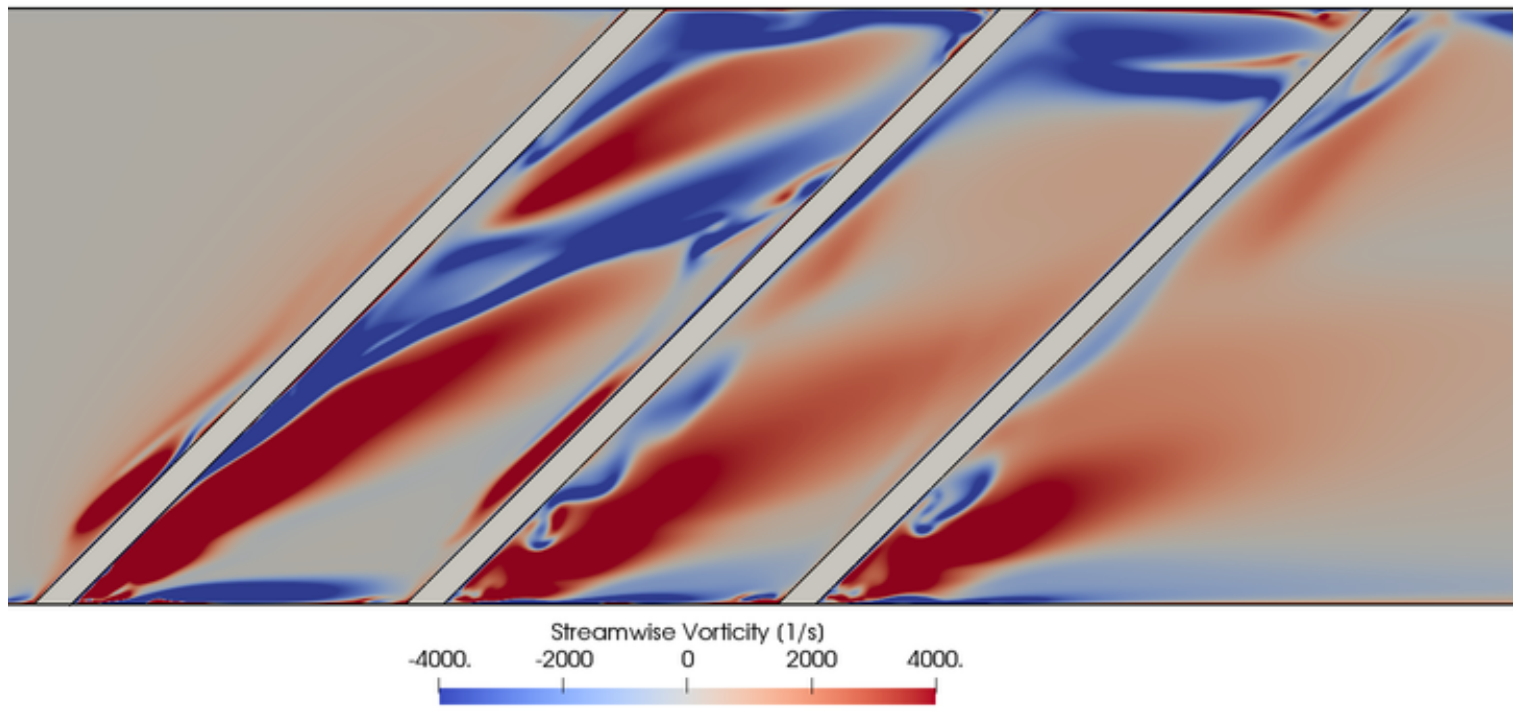

Figure B.22: Case D1 streamwise vorticity component shown on a plane section half way up the rib height. Flow moves from left to right, with positive vorticity. 


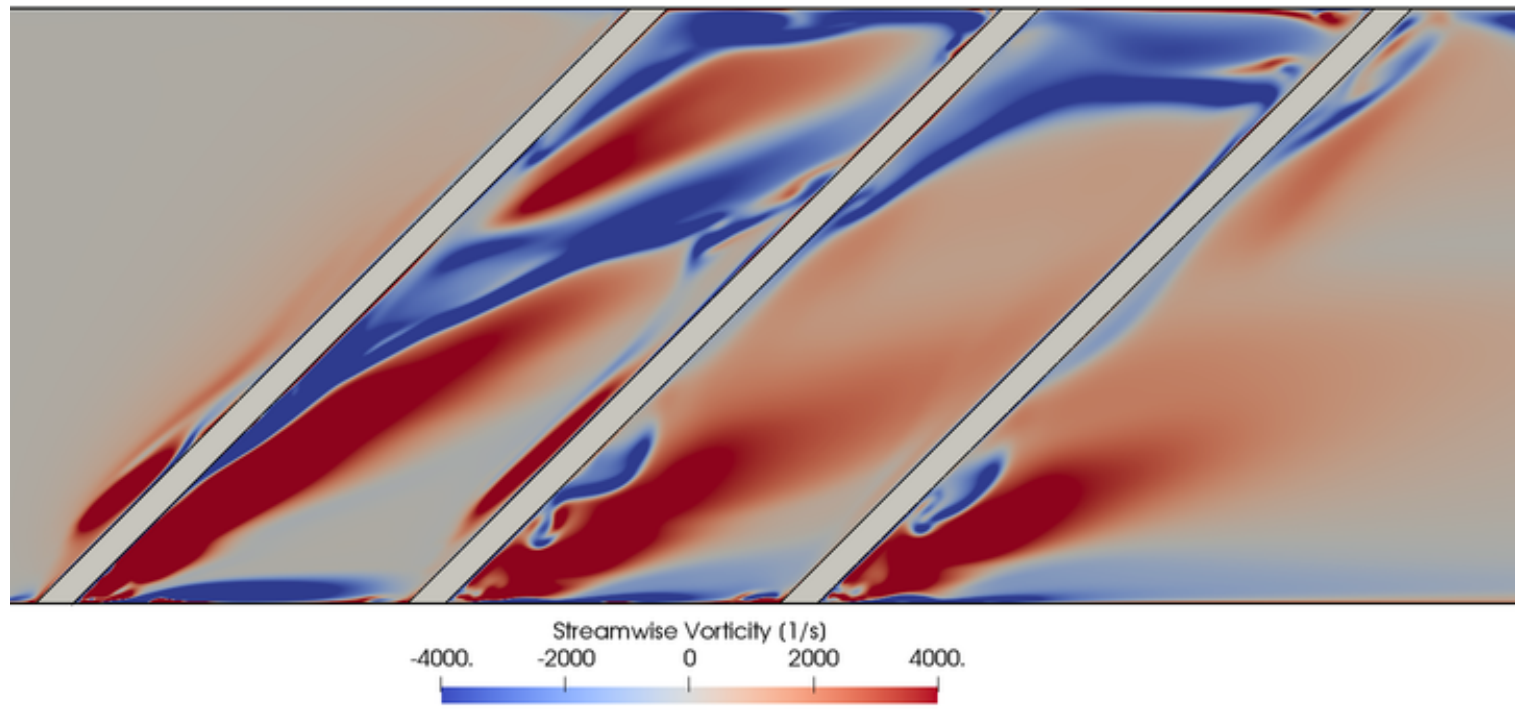

Figure B.23: Case D1 streamwise vorticity component shown on a plane section half way up the rib height. Flow moves from left to right, with positive vorticity.

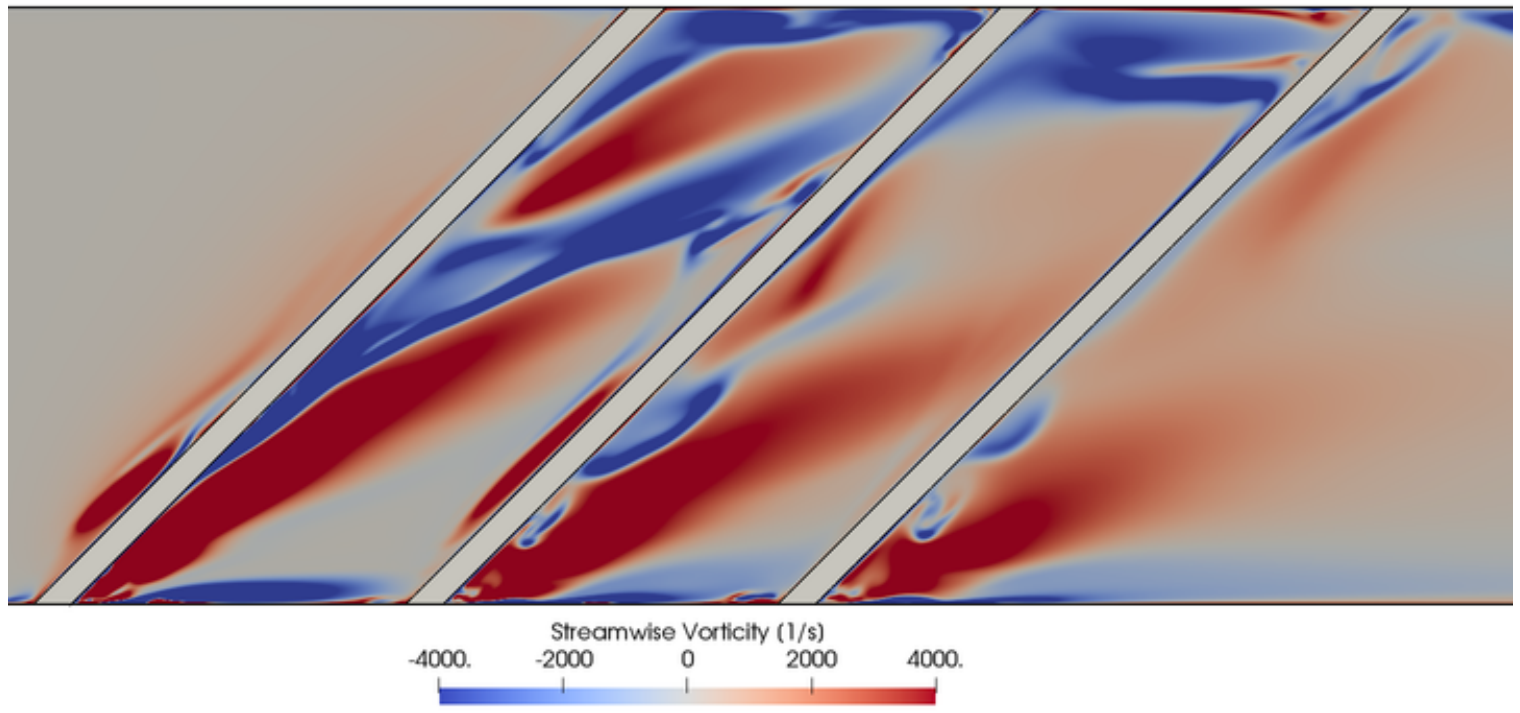

Figure B.24: Case D1 streamwise vorticity component shown on a plane section half way up the rib height. Flow moves from left to right, with positive vorticity. 


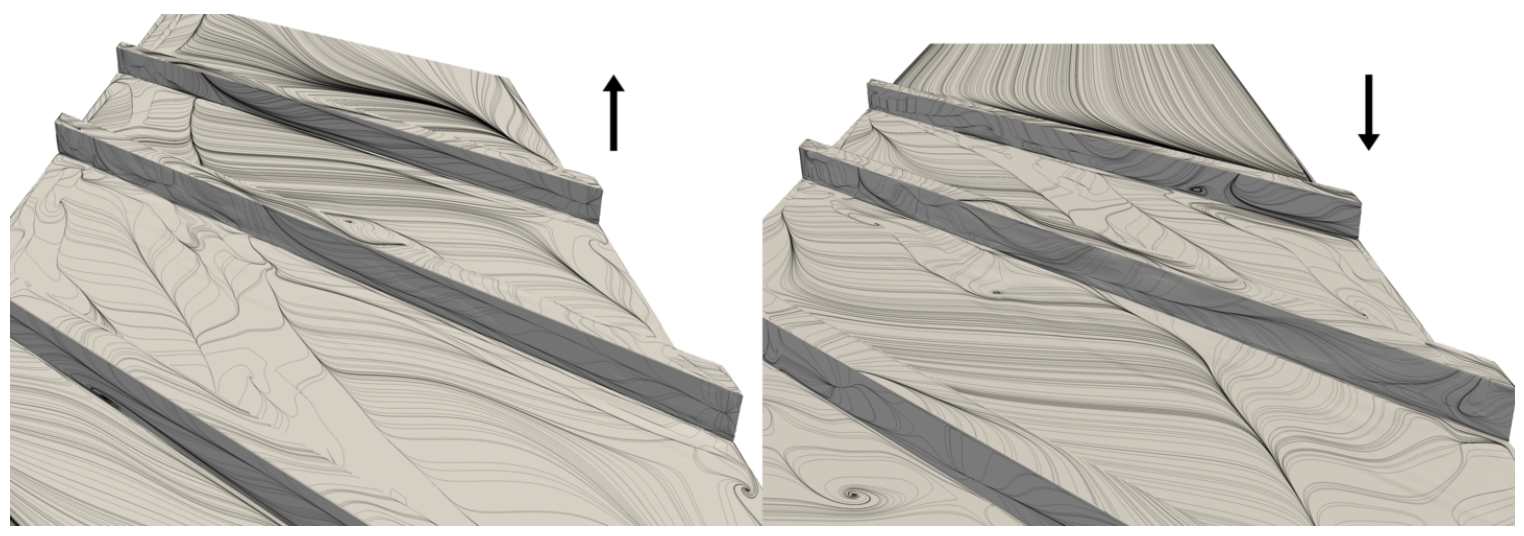

Figure B.25: Surface streamlines of case D1. As viewed from the front (left) and rear (right). Flow moves with the arrow.

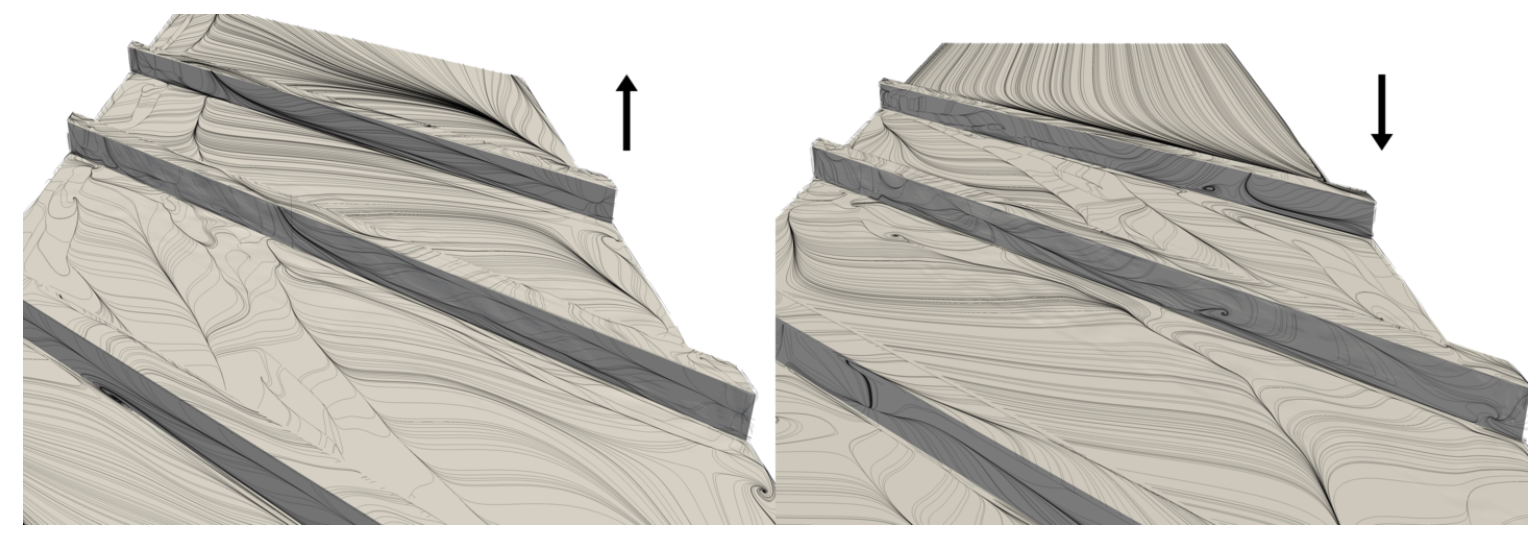

Figure B.26: Surface streamlines of case D2. As viewed from the front (left) and rear (right). Flow moves with the arrow. 


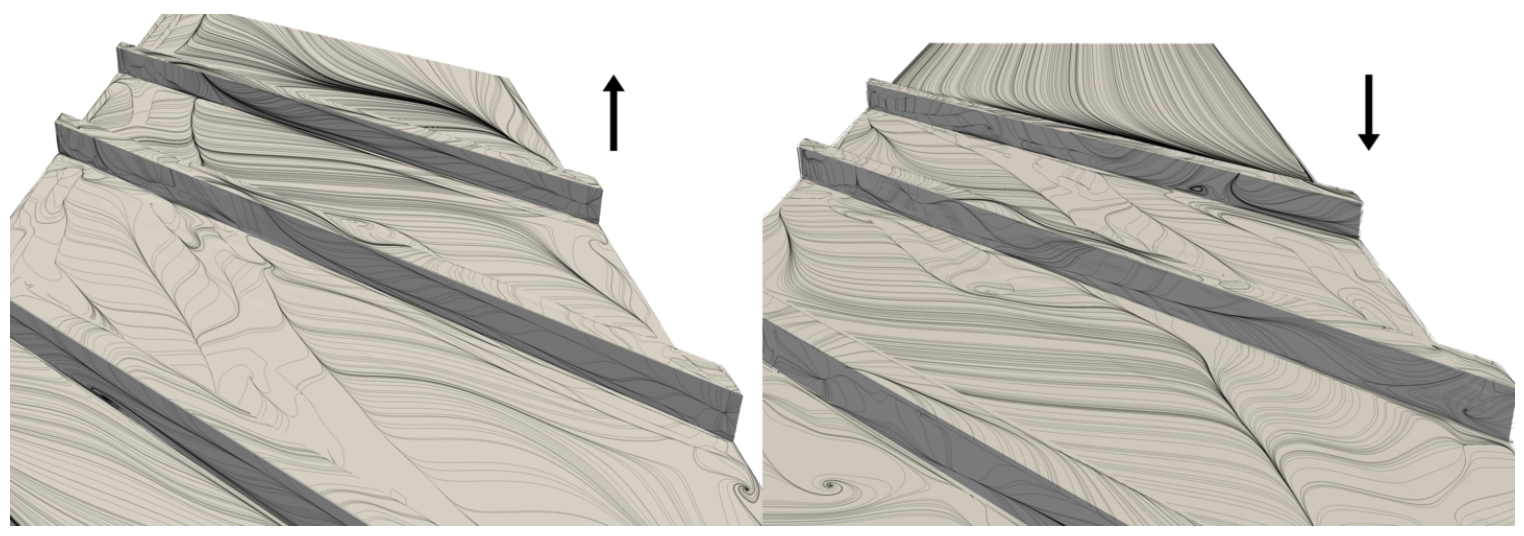

Figure B.27: Surface streamlines of case D3. As viewed from the front (left) and rear (right). Flow moves with the arrow.

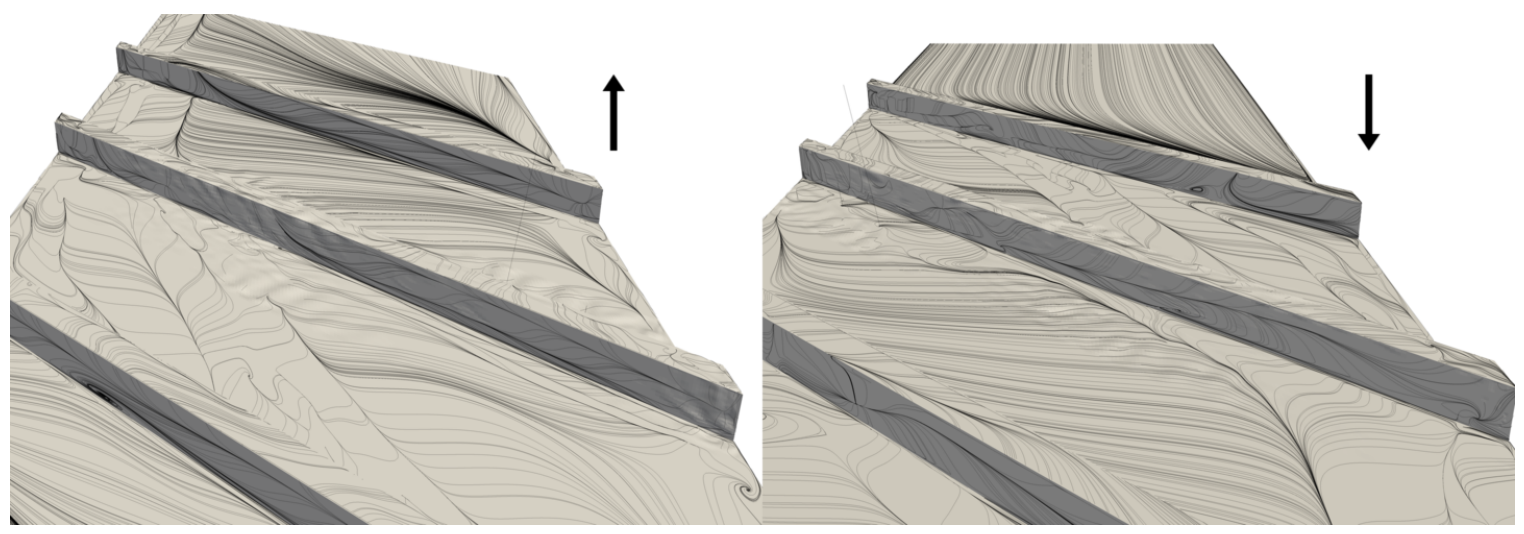

Figure B.28: Surface streamlines of case D4. As viewed from the front (left) and rear (right). Flow moves with the arrow. 


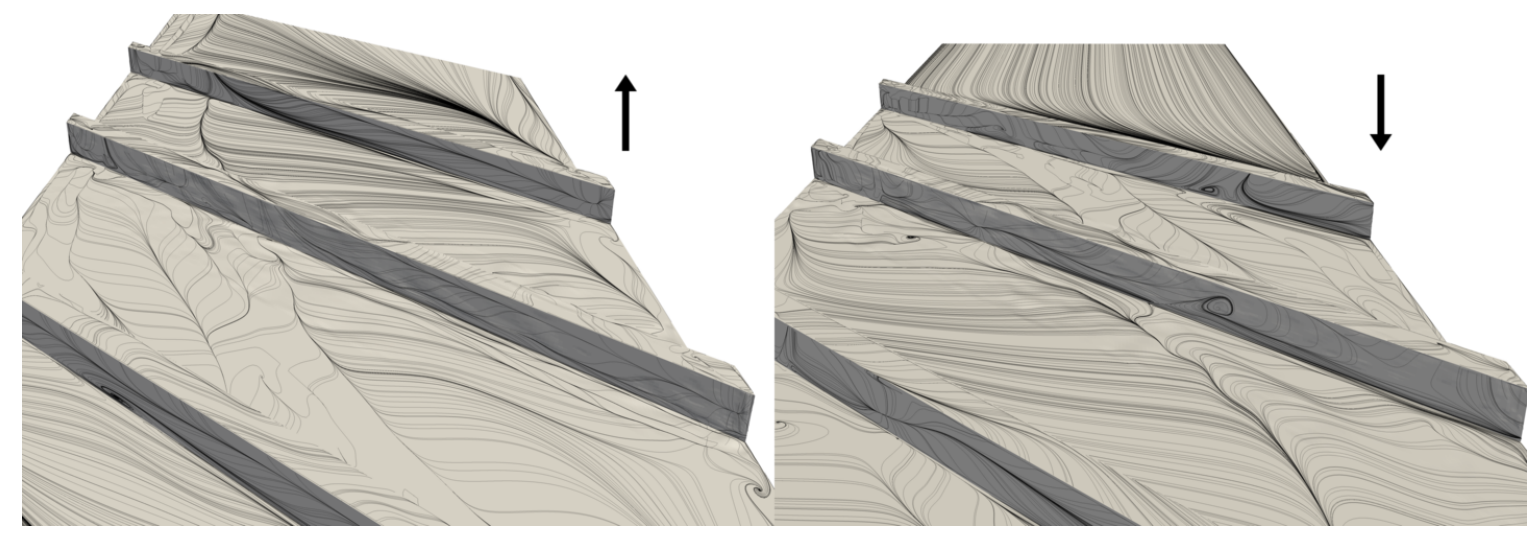

Figure B.29: Surface streamlines of case D5. As viewed from the front (left) and rear (right). Flow moves with the arrow.

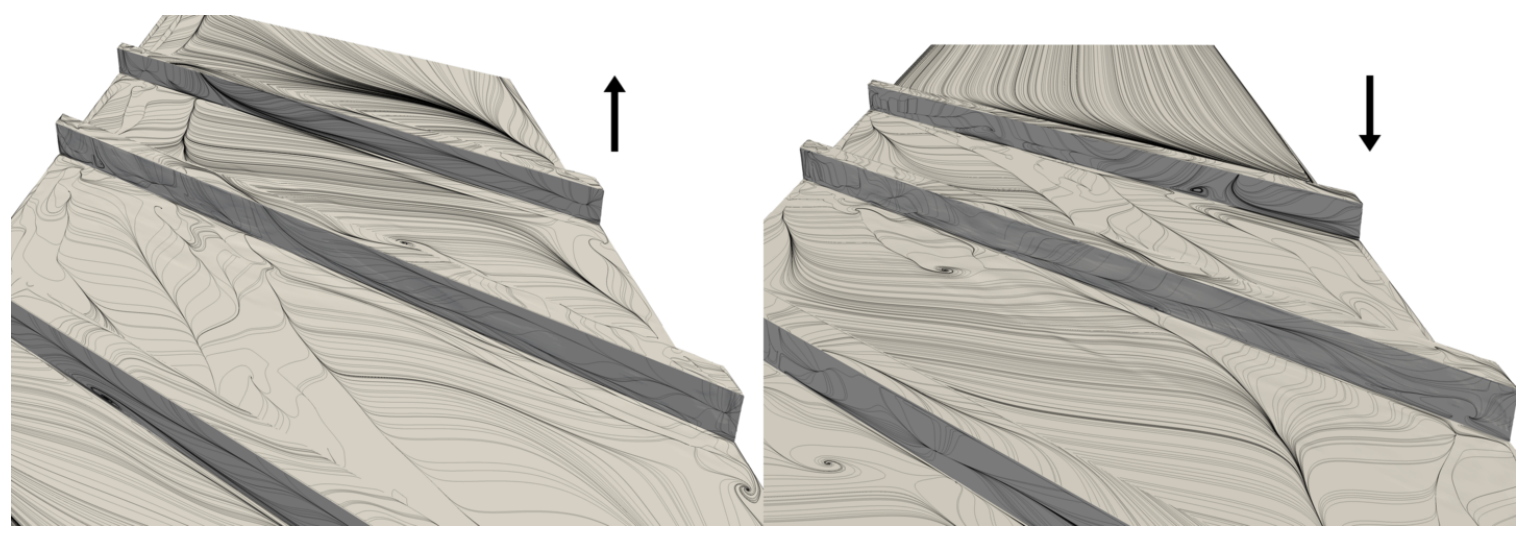

Figure B.30: Surface streamlines of case D6. As viewed from the front (left) and rear (right). Flow moves with the arrow. 
APPENDIX C: NOTES ON THE TKE RESOLUTION RATIO 
The TKE resolution ratio has been criticized as being not completely adequate by Davidson [15]. Generally, the resolved TKE involves the time-average of the turbulent velocity fluctuations and would be compared to the time-average SGS-modeled TKE. This method seems to have been accepted as a good metric by many published works. However, one can argue if the true metric for 'good' LES resolution is that of $80 \%$ of the energy must be resolved, it seems prudent that this must be applied at all instances in time. Averaging the ratio as stated above, while providing convenience from a data storage/retention standpoint, acts to smear any poorly-resolved transient features out. To find the true resolving power of the grid in question, using a TKE definition like equation C.1 may be proposed instead.

$$
k_{r e s}=\frac{1}{2}\left(u^{\prime} u^{\prime}+v^{\prime} v^{\prime}+w^{\prime} w^{\prime}\right)
$$

While calculating any fluctuating quantity still requires a time-averaged velocity field, for a stationary field these means should be constant. (In this case, as noted in section 4.2.1, these fields are not quite stationary, so this analysis is considered suspect, which is the reason for relegating it to the appendix.) Therefore the calculation of the $k_{\text {ratio }}$ with no averaging can be performed, and it must be checked at numerous timesteps during the run to ensure it meets the $80 \%$ criterion. A comparison of the two methods is shown in figure C.1. The timesteps are taken through 1 flow-through time as an example. The first point to make is that both values are low, indicating adherence to the criterion. As expected, the averaged field shows more cells in agreement with the criterion, as features which violate it have been smeared through the averaging process. The obvious negative aspect to this method is that, to improve the resolution of the grid, one must store the $k_{\text {ratio }}$ field at a particular timestep where the criterion fails in order to determine where to modify the grid. 


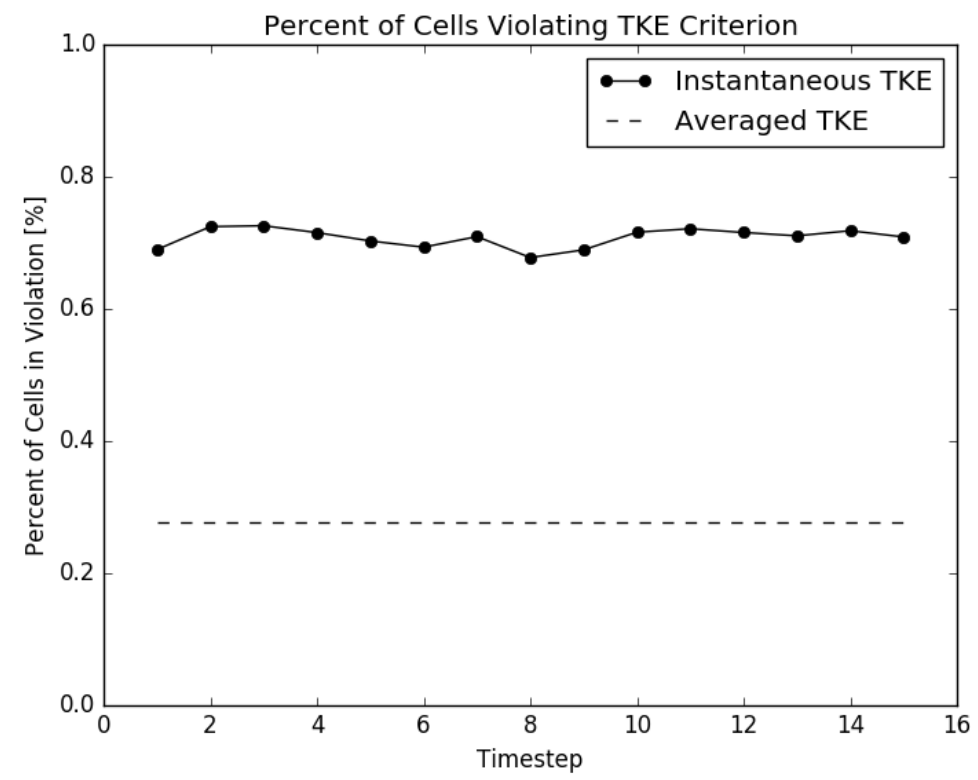

Figure C.1: Percent of cells violating the TKE resolution ratio requirement of $80 \%$. Both values are low, but using 'instantaneous' values yields a more restrictive criterion. 


\section{APPENDIX D: DETAILED COMPUTATIONAL TIME USAGE}


This section intends to give future students (and will serve as a reference for the author in the future) an idea of the computational costs associated with the simulations performed as a part of this research.

Table D.1: RANS Cases Computational Cost

\begin{tabular}{l|r|r} 
Parameter & Value & Unit \\
\hline CPU & Xeon E5-2680 @ 2.7GHz & - \\
Node count & 4 & - \\
Core count (total) & 64 & - \\
Number of iterations & 9000 & - \\
Average runtime & 76070 & $\mathrm{sec}$ \\
Average runtime & 21.1 & $\mathrm{hr}$
\end{tabular}

Table D.2: LES Case Computational Cost

\begin{tabular}{l|r|r} 
Parameter & Value & Unit \\
\hline CPU & Xeon E5-2683v3 @ 2.0GHz & - \\
Node count & 10 & - \\
Core count (total) & 280 & - \\
Number of timesteps & $1.8 \times 10^{5}$ & - \\
Total runtime & $3.7 \times 10^{6}$ & $\mathrm{sec}$ \\
Total runtime & 1034 & $\mathrm{hr}$
\end{tabular}

The DMD algorithm can be run in a "convergence" mode, where the decomposition is performed for every rank from 1 until the desired maximum, and a "industrial" mode, where the decomposition is performed merely on the maximum desired rank. Runtimes of both are presented to give perspective of what is necessary to prove convergence and what is necessary to provide engineering utility. The code utilizes several Python libraries which take advantage of multiple cores. The slowest part of the algorithm is the SVD, which is mostly serial. As shown, it benefits greatly from a CPU with a high clockspeed. Each decomposition in the "convergence" mode is done 
sequentially, however in reality they could be performed in parallel for speedup.

Table D.3: Computational cost of DMD convergence run in seconds. Runs in bold were evaluated on a machine identical to the LES case, but using only 4 cores. Otherwise runs were performed on a Core $17-5820 \mathrm{~K} @ 3.5 \mathrm{GHz}$. This table includes the data loading time from disk.

\begin{tabular}{|c|r|r|r|r|}
\hline & \multicolumn{4}{|c|}{$N$ flow-throughs } \\
\hline$\Delta t[\mu \mathrm{s}]$ & 1 & 3 & 5 & 10 \\
\hline 20 & 4055.92 & $\mathbf{6 4 6 1 3 . 3}$ & $\mathbf{1 0 6 5 7 7 . 1}$ & $\mathbf{2 1 0 9 6 1 . 0}$ \\
40 & 746.5 & 11583.2 & $\mathbf{1 0 6 1 0 8 . 2}$ & $\mathbf{1 3 9 9 0 3 . 7}$ \\
80 & 195.0 & 1982.8 & 6869.9 & $\mathbf{1 9 4 7 9 0 . 0}$ \\
160 & 73.2 & 421.9 & 1286.1 & 7421.4 \\
\hline
\end{tabular}

Table D.4: Computational cost of DMD run utlizing the maximum specified number of ranks, in seconds. Runs in bold were evaluated on a machine identical to the LES case, but using only 4 cores. Otherwise runs were performed on a Core i7-5820K @ 3.5GHz. This table does not include the data loading time from disk.

\begin{tabular}{|c|r|r|r|r|}
\hline & \multicolumn{4}{|c|}{$N$ flow-throughs } \\
\hline$\Delta t[\mu \mathrm{s}]$ & 1 & 3 & 5 & 10 \\
\hline 20 & 92.2 & $\mathbf{6 8 3 . 7}$ & $\mathbf{1 3 1 0 . 8}$ & $\mathbf{2 6 8 0 . 2}$ \\
40 & 25.0 & 178.1 & $\mathbf{1 2 8 7 . 5}$ & $\mathbf{1 7 2 4 . 6}$ \\
80 & 7.6 & 52.8 & 123.8 & $\mathbf{2 0 6 0 . 9}$ \\
160 & 3.0 & 15.0 & 37.5 & 130.0 \\
\hline
\end{tabular}


Table D.5: Memory requirements of DMD convergence run in GB.

\begin{tabular}{|c|r|r|r|r|}
\hline & \multicolumn{4}{|c|}{$N$ flow-throughs } \\
\hline$\Delta t[\mu \mathrm{s}]$ & 1 & 3 & 5 & 10 \\
\hline 20 & 8.7 & $\mathbf{2 5 . 6}$ & $\mathbf{3 0 . 8}$ & $\mathbf{4 4 . 0}$ \\
40 & 4.4 & 13.0 & $\mathbf{3 0 . 9}$ & $\mathbf{3 5 . 3}$ \\
80 & 2.3 & 6.6 & 11.0 & $\mathbf{2 6 . 4}$ \\
160 & 1.2 & 3.4 & 5.5 & 10.8 \\
\hline
\end{tabular}


APPENDIX E: DMD CODE NOTES 
This section reviews the necessary items and packages in order to run the DMD code in appendix F. This Python script is the main DMD code utilized for this paper, and does a majority of the post-processing. It is designed to be executed on GNU/Linux using Python 2.7.12. Check the imports for required packages. It runs image generation in software, so it will work even on machines with no $\mathrm{X}$ server. The inputs are explained in the code after the function definitions. The user can be reminded of the inputs by executing the code with no arguments; the required inputs will be printed to the terminal.

Point probe data is expected to be in a single directory using the OpenFOAM point cloud format. The directory should be named ribNprobes, where $\mathrm{N}$ is the rib number, going from 1 to 3 . Although the point data (in other words, the grid) is read from file and loaded into memory, the grid is reproduced with a separate function in the DMD code anyway, merely for historical reasons.

The point probes are in a block-structured set with one block in front of, cornered to, on top of, rear cornered, and behind the rib. For another geometry the different data structure mapping functions moving from the block-structured to fully structured formats must be re-written. After initially creating blocked formats, it is advised new users instead build structured formats from the beginning and convert to blocks only for post-processing purposes.

Custom colormaps must be specified explicitly by filename using the ParaView XML colormap format.

The DMD code has 2 major operating modes. One is the standard ("std") mode, which executes the DMD algorithm starting from a single rank and sweeps through all ranks until the specified maximum. This mode is most useful for convergence runs. A 'single-rank' mode is available which will only run a desired rank for speed. The second mode is the per-rank heat transfer ("per") mode. This mode executes a single-rank run for the desired rank to generate the DMD modes. Following, the heat transfer field is recomputed for as many times as the user specifies, increasing the number of ranks in the computation by 2 each time. This mode is useful for determining the heat transfer value in each mode. 
APPENDIX F: DMD CODE 
NOTE: Originally I was to place the entire code here, but it seems silly to do so considering the power of the Internet. The user may get a copy of the code by sending me an email at elmoremt@knights.ucf.edu 


\section{LIST OF REFERENCES}

[1] Abdel-Wahab, S., and Tafti, D. K., "Large Eddy Simulation of Flow and Heat Transfer in a Staggered 45 Ribbed Duct”, Proceedings of ASME Turbo Expo, 2004.

[2] Ahmed, L., Vergos, C., Tran, P. K., Wang, W., and Kapat, J. S., "Investigation of Pressure Drop and Heat Transfer Behavior of a Square Channel with 45 Angle Ribs at Wide Range of Reynolds Numbers", 51st AIAA/SAE/ASEE Joint Propulsion Conference, 2015.

[3] Ali, M. S., Tariq, A., and Gandhi, B. K., "Role of Chamfering Angles and Flow Through Slit on Heat Transfer Augmentation Behind a Surface-Mounted Rib”, Journal of Heat Transfer, vol. 138, 2016.

[4] Bailey, J. C., and Bunker, R. S., "Heat transfer and friction in channels with very high blockage 45 staggered turbulators", ASME Turbo Expo, 2003.

[5] Beck, T. W., Housh, T. J., Weir, J. P., Cramer, J. T., Vardaxis, V., Jonson, G. O., Coburn, J. W., Malek, M. H., and Mielke, M., "An examination of the Runs Test, Reverse Arrangements Tests, and modified Reverse Arrangements Test for assessing surface EMG signal stationarity”, Journal of Neuroscience Methods, vol. 156, pp. 242-248, 2006.

[6] Bendat, J. S. and Piersol, A. G., Random Data. Hoboken, NJ: John Wiley \& Sons, 2010.

[7] Berkooz, G., Holmes, P., and Lumley, J. L., "The proper orthogonol decomposition in the analysis of turbulent flows", Annual Review of Fluid Mechanics, vol. 25, pp. 539-575, 1993.

[8] Cardwell, N. D., Vlachos, P. P., and Thole, K. A., "Developing and fully developed turbulent flow in ribbed channels", Experimental Fluids, vol. 50, pp. 1357-1371, 2011. 
[9] Chandra, P. R., Niland, M. E., Han, J. C., "Turbulent flow heat transfer and friction in a rectangular channel with varying numbers of ribbed walls", Journal of Turbomachinery, vol. 119, pp. 374-380, 1997.

[10] Chanteloup, D., Jauneda, Y., and Boics, A., "Combined 3D flow and heat transfer measurements in a 2-pass internal coolant passage of gas turbine airfoils", ASME Turbo Expo, 2002.

[11] Coletti, F., Jacono, D. L., Cresci, I., and Arts, T., "Turbulent flow in rib-roughened channel under the effect of Coriolis and rotational buoyancy forces", Physics of Fluids, vol. 26, 2014.

[12] Dritselis, C. D., "Large eddy simulation of turbulent channel flow with transverse roughness elements on one wall", Intl Journal of Heat and Fluid Flow, vol. 50, pp. 225-239, 2014.

[13] Ferziger, J. H., and Peric, M., Computational Methods for Fluid Dynamics. Berlin: SpringerVerlag, 2002.

[14] Forsyth, P., McGilvray, M., and Gillespie, D. R. H., "Secondary flow and heat transfer coefficient distributions in the developing flow region of ribbed turbine blade cooling passages", Journal of Experimental Fluids, vol. 58, no. 5, 2017.

[15] Davidson, L., "Large Eddy Simulations: how to evaluate resolution", Intl. Journal of Heat and Fluid Flow, vol. 30, no. 5, pp. 1016-1025, 2009.

[16] Grenga, T., MacArt, J. F., and Mueller, M. E., "Dynamic mode decomposition of a direct numerical simulation of a turbulent premixed planar jet flame: convergence of the modes", Combustion Theory and Modelling, vol. 22, no. 4, pp. 795-811, 2018.

[17] Han, J. C., Chen, H., "Turbine Blade Internal Cooling Passages with Rib Turbulators", Journal of Propulsion and Power, vol. 22, no. 2, pp. 226-248, 2005. 
[18] Han, J. C., Park, J. S., and Lei, C. K., "Heat Transfer Enhancement in Channels with Turbulence Promoters", Journal of Engineering for Gas Turbines and Power, vol. 107, pp. 628-635, 1985 .

[19] Hossain, M. S., Bergstrom, D. J., and Chen, X.B., "Visualisation and analysis of large-scale fortex structures in three-dimensional turbulent lid-driven cavity flow", Journal of Turbulence, vol. 16 No.10, pp. 901-924, 2015.

[20] Hu, H., Du, X., Yang, L, and Yang, Y., "POD based modeling on flow and heat transfer of air-cooled condenser influenced by natural wind", Int. Journal of Heat and Mass Transfer, vol. 74, pp. 431-440, 2014.

[21] Jovanovi, M. R., Schmid, P. J., and Nichols, J. W., "Sparsity-promoting dynamic mode decomposition”, Physics of Fluids, vol. 26, 2014.

[22] Kalghatgi, P. and Acharya, S., "Modal Analysis of Inclined Film Cooling Jet Flow", Journal of Turbomachinery, vol. 136, 2014.

[23] Kang, C. and Yang, K., "Characterization of turbulent heat transfer in ribbed pipe flow", Journal of Heat Transfer, vol. 138, 2016.

[24] Kunstmann, S., Wolfersdorf, J., Ruedel, U., 'Heat Transfer and Pressure Loss in Rectangular One-Side-Ribbed Channels with Different Aspect Ratios", Journal of Turbomachinery, vol. $135,2013$.

[25] Kutz, N. J., Brunton, S. L., Brunton, B. W., and Proctor, J. L., Dynamic Mode Decomposition. Philadelphia, PA: Society for Industrial and Applied Mathematics, 2016.

[26] Labbe, O., "Large-eddy-simulation of flow and heat transfer in a ribbed duct", Comptuers \& Fluids, vol. 76, pp. 23-32, 2013. 
[27] Li, Z., Xu, X., Li, K., Chen, Y., Huang, G., Chen, C., and Chen, C., ”A flapping vortex generator for heat transfer enhancement in a rectangular airside fin", Intl Journal of Heat and Mass Transfer, vol. 118, pp. 1340-1356, 2018.

[28] Liou, T. M., Chang, S. W., Lan, Y. A., Chan, S. P., Liu, Y. S., ”Heat transfer and flow characteristics of two-pass parallelogram channels with attached and detached transverse ribs", Journal of Heat Transfer, vol. 139, 2017.

[29] Mahapatra, P. S., Chatterjee, S., Mukhopadhyay, A. and Manna, N. K., "Proper orthogonal decomposition of thermally-induced flow structure in an enclosure with alternately active localized heat sources", Int. Journal of Heat and Mass Transfer, vol. 94, pp. 373-379, 2016.

[30] Mhetras, S., Han, J. C., and Huth, M., "Heat Transfer and Pressure Loss Measurements in a Turbulated High Aspect Ration Channel with Large Reynolds Number Flows”, Journal of Thermal Science and Engineering Applications, vol. 6, 2014.

[31] Muld, T. W., Efraimsson, G., and Henningson, D. S., "Flow Structures around a high-speed train extracted using Proper Orthogonal Decomposition and Dynamic Mode Decomposition”, Computers and Fluids, vol. 57, pp. 87-97, 2012.

[32] Nicoud, F., and Ducros, F., "Subgrid-scale stress modelling based ont eh square of the velocity gradient tensor", Flow, Turbulence and Combustion, vol. 62, pp. 183-200, 1999.

[33] Park, J., Park, S., and Ligrani, P.M., "Numerical predictions of detailed flow structural characteristics in a channel with angled rib turbulators", Journal of Mechanical Science and Technology, vol. 29, pp. 4981-4991, 2015.

[34] Park, J. S., Han, J. C., Huang, Y., and OU, S., "Heat transfer performance comparisons of five different rectangular channels with parallel angled ribs", Intl Journal of Heat and Mass Transfer, vol. 35 no. 11, pp. 2891-2903, 1992. 
[35] Park, T., Kursat, K., and Kim, D., "Flow structure and heat transfer of a sweeping jet impinging on a flat wall”, Int. Journal of Heat and Mass Transfer, vol. 124, pp. 920-928, 2018.

[36] Patil, S., and Taffi, D., "Large-Eddy Simulation With Zonal Near Wall Treatment of Flow and Heat Transfer in a Ribbed Duct for the Internal Cooling of Turbine Blades", Journal of Turbomachinery, vol. 135, 2013.

[37] Piomelli, U., and Chasnov, J., "Large Eddy Simulation: Theory and Applications", Turbulence and Transition Modeling, pp. 269-336, 1996.

[38] Pope, S. B., Turbulent Flows. Cambridge, NY: Cambridge University Press, 2000.

[39] Rallabandi, A. P., Yang, H., and Han, J. C., "Heat transfer and pressure drop correlations for square channels with 45 deg ribs at high reynolds numbers", Journal of Heat Transfer, vol. $131,2009$.

[40] Rau, G., Caken, M., Moeller, D. and Arts, T., "The effect of periodic ribs on the local aerodynamic and heat transfer performance of a straight cooling channel", Intl. Gas Turbine and Aeroengine Congress \& Expo, 1996.

[41] Rowley, C. W., Mezic, I., Bagheri, S., Schlatter, P., and Henningson, D. S., "Spectral analysis of nonlinear flows", Journal of Fluid Mechanics, vol. 641, pp. 115-127, 2009.

[42] Saidi, A., and Sunden, B., "On prediction of thermal-hydraulic characteristics of squaresectioned ribbed cooling ducts", Journal of Turbomachinery, vol. 123, pp. 614-620, 2001.

[43] Saha, A. K., and Acharya, S., "Turbulent heat transfer in ribbed coolant passages of different aspect ratios: parametric effects", Journal of Heat Transfer, vol. 129, pp. 449-463, 2007.

[44] Saha, P. Biswas, G., Mandal, A. C., and Sarkar, S., "Investigation of coherent structures in a turbulent channel with built-in longitudinal vortex generators", Int. Journal of Heat and Mass Transfer, vol. 104, pp. 178-198, 2017. 
[45] Sayadi, T., Schmid, P. J., Nichols, J. W., and Moin, P., "Reduced-order representation fo nearl-wall structures in the late transitional boundary layer", Journal of Fluid Mechanics, vol. 748, pp. 278-301, 2014.

[46] Schmid, P. J., Meyer, K. E., and Pust, O., ”Dynamic Mode Decomposition and Proper Orthogonal Decomposition of flow in a lid-driven cylindrical cavity", 8th Intl Symposium on Particle Image Velocimetry, Melbourne, Victoria, Australia, 2009.

[47] Schmid, P. J., Li, L., Juniper, M. P., and Pust, O., "Applications of the dynamic mode decomposition”, Theoretical Computational Fluid Dynamics, vol. 25, pp. 249-259, 2011.

[48] Schmid, Peter. "Dynamic mode decomposition of numerical and experimental data", Journal of Fluid Mechanics, vol. 656, pp. 5-28, 2010.

[49] Schmitt, T., Ruiz, A., Selle, L. and Cuenot, B., "Numerical Investigation of the Destablization of Supercritical Round Turbulent Jets using Large-Eddy Simulation”, Progress in Propulsion Physics, vol. 2, pp. 225-238, 2011.

[50] Schwanen, M. and Duggleby, A., "Optimizing turbulent heat transfer using orthogonal decomposition-based contouring", Journal of Turbulence, vol. 12, No. 31, pp. 1-20, 2011.

[51] Sewall, E. A., Tafti, D. K., Graham, A. B., and Thole, K. A., 'Experimental validation of large eddy simulations of flow and heat transfer in a stationary ribbed duct", Intl. Journal of Heat and Fluid Flow, vol. 27, pp. 243-258, 2006.

[52] Singh, P., Ravi, B. V., and Ekkad, S. V., "Experimental and numerical study of heat transfer due to developing flow in a two-pass rib roughened square duct", Intl Journal of Heat and Mass Transfer, vol. 102, pp. 1245-1256, 2016. 
[53] Son, S. Y., Kihm, K. D., and Han, J. C., "PIV flow measurements for heat transfer characterization in two-pass square channels with smooth and 90 ribbed walls", Intl Journal of Heat and Mass Transfer, vol. 45, pp. 4809-4822, 2002.

[54] Taslim, M. E., and Korotky, G. J., "Low-aspect-ration rib heat transfer coefficient measurements in a square channel", Journal of Turbomachinery, vol. 120, pp. 831-838, 1998.

[55] Taslim, M. E., and Wadsworth, C. M., "An experimental investigation of the rib surfaceaveraged heat transfer coefficient in a rib-roughened square passage", Intl. Gas Turbine and Aeroengine Congress and Expo, 1994.

[56] Tyagi, M., and Acharya, S., "Large Eddy Simulations of Flow and Heat Transfer in Rotating Ribbed Duct Flows", Journal of Heat Transfer, vol. 127, pp. 486-498, 2005.

[57] Valentino, M. I., Tran, L. V., Ricklick, M., and Kapat, J. S., ”Comparison of Heat Transfer and Friction Augmentation for Symmetric and Non-Symmetric Wedge Turbulators on Two Opposite Walls", 47th AIAA/ASME/SAE/ASEE Joint Propulsion Conference, 2011.

[58] Wen, X., Liu, Y., and Tang, H., "Unsteady behavior of a sweeping impinging jet: Timeresolved particle image velocimetry measurements", Experimental Thermal and Fluid Science, vol. 96, pp. 111-127, 2018.

[59] Wenwu, Z., Peng, D., Wen, X., Yingzheng, L., and Hu, H., ”Unsteady analysis of adiabatic film cooling effectiveness behind circular, shaped, and sand-dune-inspired film cooling holes: Measurement using fast-response pressure-sensitive paint", Intl Journal of Heat and Mass Transfer, vol. 125, pp. 1003-1016, 2018.

[60] Wright, L. M., Fu, W. L., and Han, J. C., ’Thermal Performance of Angled, V-shaped, and Wshaped Rib Turbulators in Rotating Rectangular Cooling Channels (AR=4:1)", Proceedings of ASME Turbo Expo, 2004. 
[61] Xie, G., Li, S., Zhang, W., and Sunden, B., "Computational Fluid Dynamics Modeling Flow Field and Side-Wall Heat Transfer in Rectangular Rib-Roughened Passages", Journal of Energy Resources Technology, vol. 135, 2013.

[62] Zhang, Q., Liu, Y., and Wang, S., "The identification of coherent structures using proper orthogonal decomposition and dynamic mode decomposition", Journal of Fluids and Structures, vol. 49, pp. 53-72, 2014. 